\title{
Periostin - a biomarker in adults with
}

\section{asthma}

By Ruth Semprini

A thesis submitted to Victoria University of Wellington in fulfilment of the requirements for the degree of Doctor of Philosophy

Victoria University of Wellington 


\section{Abstract}

Asthma is a common, heterogeneous condition where current treatment options are limited to a 'one size fits all' approach. Biological therapies targeting specific components of the Type 2 inflammatory pathway are emerging as potential alternatives for those who are inadequately controlled on current treatment options. Biomarkers, such as serum periostin, can be used in clinical settings to identify potential responders to these treatments.

The aim of this research was to investigate the epidemiology of periostin and its ability to predict important clinical outcomes in asthma.

Six studies were conducted: two cohort studies observing the change to periostin after bone and dental injury in non-asthmatic adults; a cohort study investigating reference ranges of periostin in Chinese adults with and without asthma; two longitudinal studies measuring the change in periostin in asthmatic adults with stable and unstable disease; and a longitudinal cohort study investigating the association between periostin and risk of exacerbation.

There was a biphasic response of serum periostin after bone injury. This was particularly marked after joint replacement surgery where periostin fell within 48 hours by a ratio of geometric means $0.80(95 \% \mathrm{Cl} 0.75$ to 0.86$)$ before rising to a maximum level at eight weeks with a ratio of geometric means $1.89(95 \% \mathrm{Cl} 1.77$ to 
2.02). There was no significant change to periostin after simple or surgical tooth extractions with a maximal ratio of geometric means of 1.02 ( $95 \% \mathrm{Cl} 0.95$ to 1.10 ). Serum periostin was higher in Chinese non-asthmatic adults versus Caucasian nonasthmatic adults, mean periostin $57 \mathrm{ng} / \mathrm{ml}$ and $49.7 \mathrm{ng} / \mathrm{ml}$ respectively, difference $(95 \% \mathrm{Cl}) 8.2(5.8$ to 10.6$) \mathrm{ng} / \mathrm{ml}$. Serum periostin remained stable in adults with wellcontrolled asthma with an intra-class coefficient for variation of 0.93 . In unstable asthma, there was a decrease in serum periostin one week after the start of a severe exacerbation and treatment with systemic corticosteroids, with a ratio of geometric means $0.86(95 \% \mathrm{Cl} 0.82$ to 0.92$)$ before stabilising four weeks later. Finally, adults with mild to moderate asthma with low baseline levels of periostin were more likely to have a severe asthma exacerbation with a hazard ratio $(95 \% \mathrm{Cl}) 0.62(0.35$ to 1.09$)$ per $0.693 \mathrm{ng} / \mathrm{ml}$ increase of log periostin.

In conclusion, serum periostin showed significant biphasic variation in response to bone injury, the magnitude and duration of which was proportional to bone size. Whilst this pattern was not replicated in adults undergoing dental surgery, it suggests that serum periostin can be affected by non-asthma related conditions. Periostin demonstrated higher mean values in Chinese adults than in Caucasian adults, indicating ethnicity-specific reference ranges may be required if it were to become a clinical biomarker. Intra-participant variability of serum periostin was low in a homogenous group of well controlled, moderate to severe asthmatic adults, but there was wide variability between individuals in this group suggesting that factors other than asthma are likely to affect serum periostin levels. Serum periostin was suppressed during and after treatment for a severe exacerbation for up to four weeks. 
This is likely due to the exacerbation and/or its treatment, suggesting the interpretation of periostin as a biomarker for response to biological therapies should not occur within four weeks of a severe exacerbation. The reported positive association between periostin and risk of severe exacerbation in populations with severe eosinophilic asthma does not extend to a general population of mild to moderate asthmatics, in which an inverse associated was observed.

Serum periostin may be useful in predicting treatment responsiveness of patients to monoclonal antibody therapy directed against IL-4R $\alpha$, IL-13 and IgE. However, it may be difficult to use as a biomarker clinically, as numerous non-asthma related factors, such as bone injury and ethnicity, have been shown to significantly affect serum levels, making interpretation difficult.

(616 of 500 words) 


\section{Acknowledgements}

I would like to thank my primary supervisor, Richard Beasley, who's limitless energy and enthusiasm for research has been a source of inspiration to me from the moment I started working at the MRINZ. Without him, I would not be submitting a thesis at all.

Professor Mark Weatherall, who's patience and sympathy at my complete and utter ineptitude in all things statistics-related has been greatly appreciated!

My supervisor Professor John Miller, who waded through drafts and ideas and offered me advice, support and help when needed.

All the staff at the MRINZ, who have been beyond fantastic, but in particular: Mathew Williams, who taught me everything I will ever need to know about spirometers, FeNO machines and filters; Irene Braithwaite who kept me sane, entertained me when I was desperate and plied me with chocolate; Nick Shortt, Stefan Ebmeier and Rachel Varughese, who helped me with my countless patient visits; Ali Pritchard who always always sorted my computer out when it decided to have a fail day and Denise Fabian, who graciously spotted formatting mistakes/typos and never made me feel silly about them.

Thanks to my co-authors, in particular Cecile Holweg of Genentech in the USA who's feedback and quick turn around of manuscripts has enabled me to keep the conveyor belt moving. 
My parents in the UK who have encouraged and supported me throughout this PhD from afar, and who never doubted me.

Last but not least, thank you to my family. My husband, Alex, who has kept me fed, watered and child-free, when I so desperately needed to write, sleep or think. My children, Jack, Rosie and Tilly, who have been a constant source of joy (and sleep deprivation) during the latter part of this research. They make completing things like this all the more precious!

Thank you 


\section{Table of Contents}

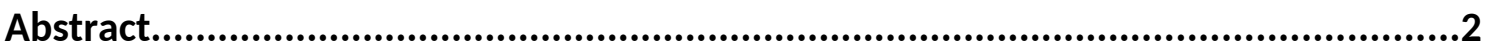

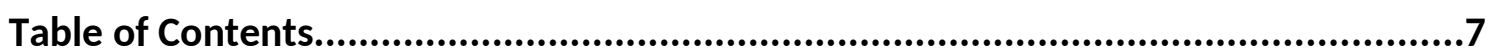

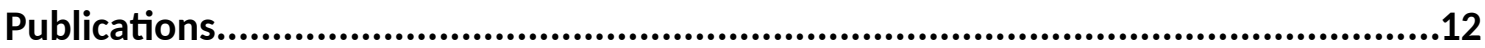

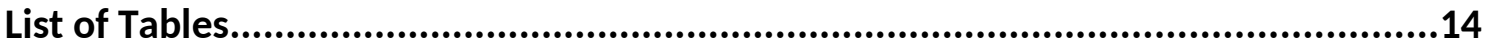

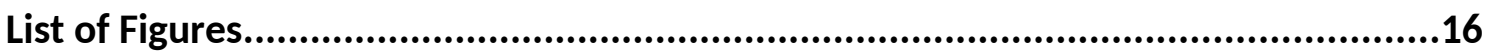

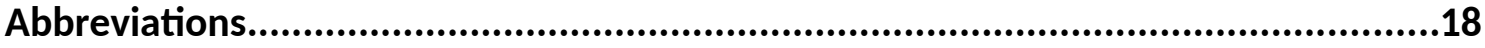

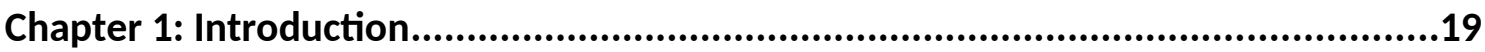

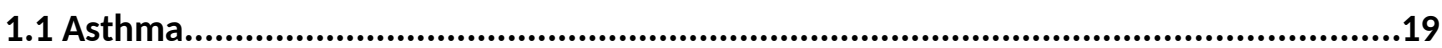

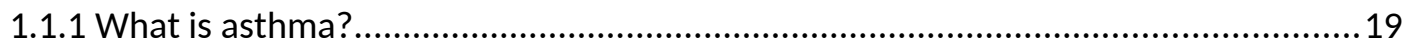

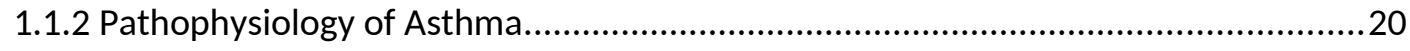

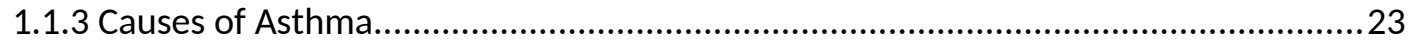

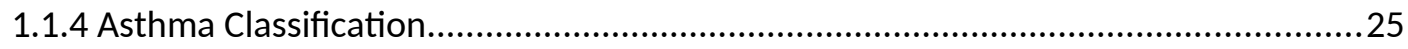

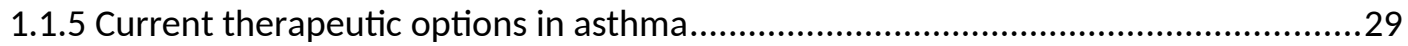

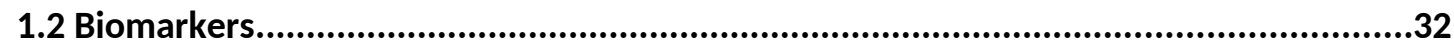

1.2.1 What is a biomarker?

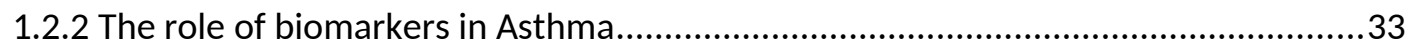

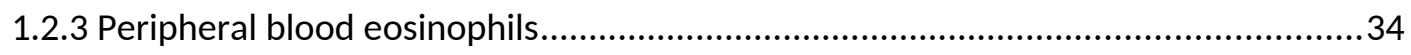

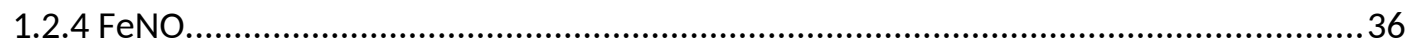

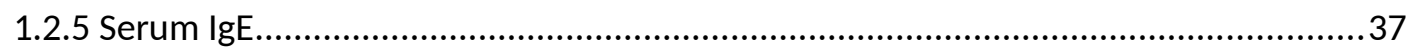

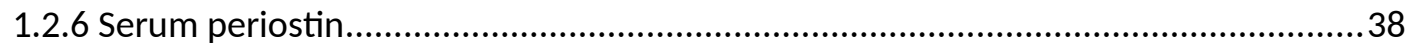

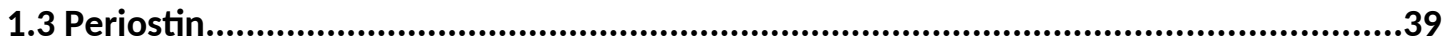




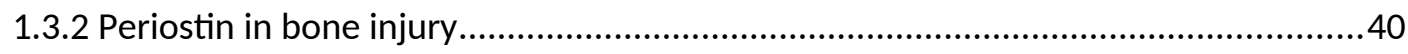

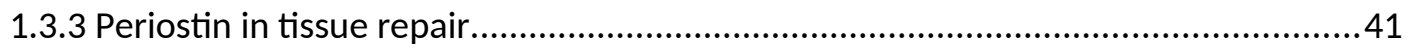

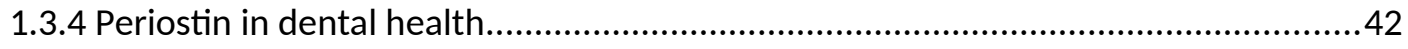

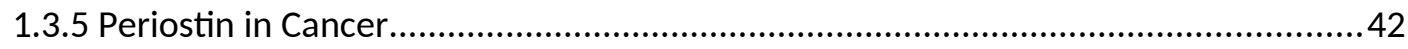

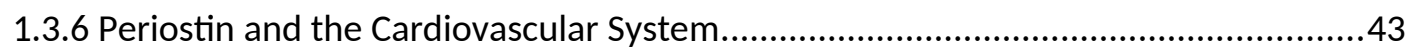

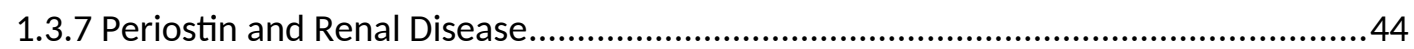

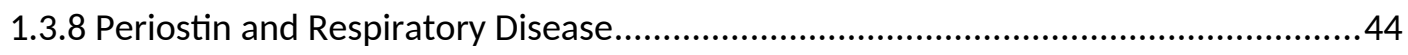

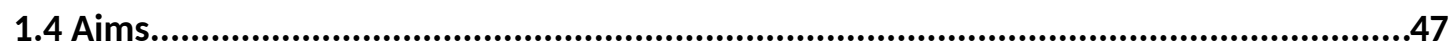

1.5 Thesis Outline

Chapter 2: Variations in serum periostin after bone and dental injury....................50

2.1 What we know so far regarding periostin in bone growth and healing........................50

2.2 What we know so far about periostin in dental health..........................................52

2.3 Study One: Change in serum periostin after joint replacements, long bone fractures or

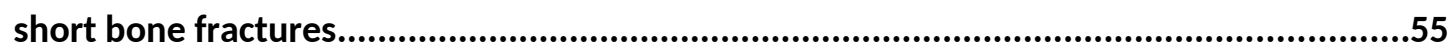

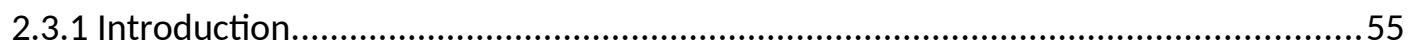

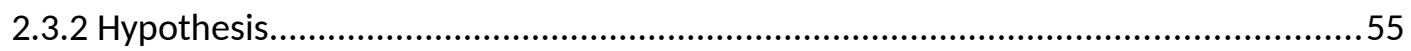

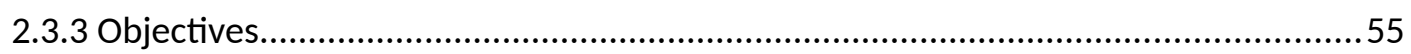

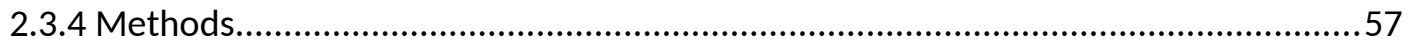

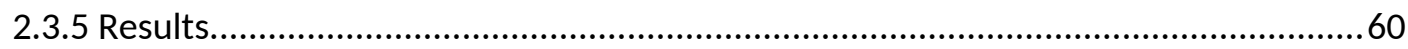

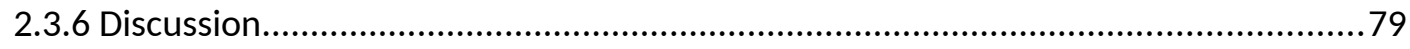

2.4 Study Two: Change in serum periostin after tooth extraction.......................................83

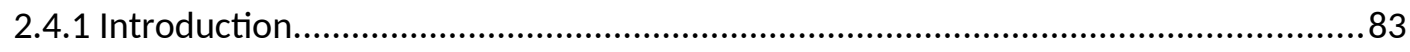

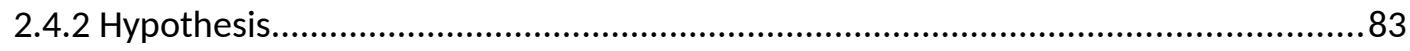

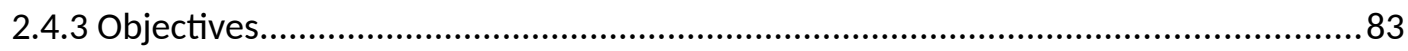

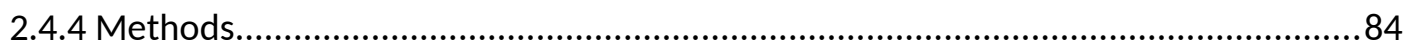




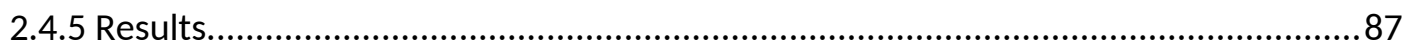

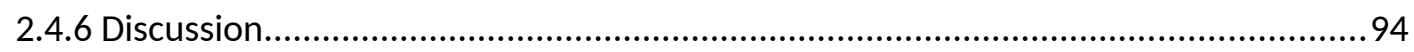

Chapter 3: Periostin as a biomarker.............................................................96

3.1 Study Three: Serum periostin levels in adults of Chinese descent...............................99

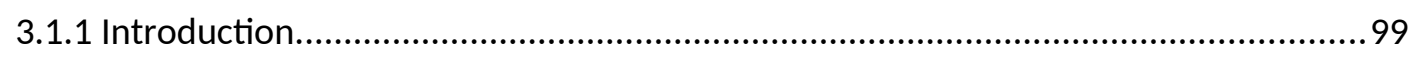

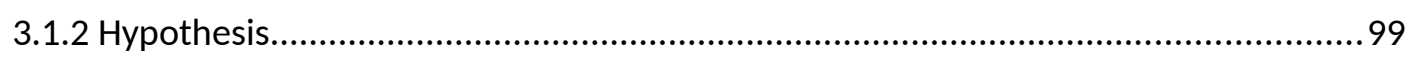

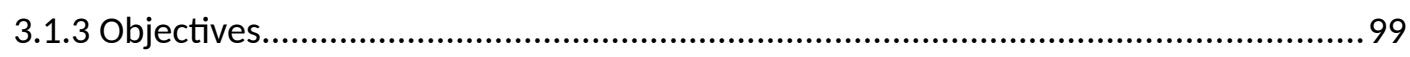

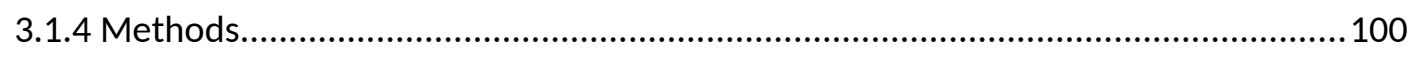

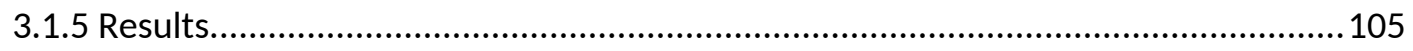

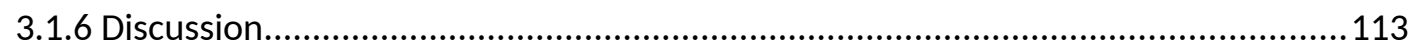

3.2 Study Four: Longitudinal variation of serum periostin in adults with stable asthma..118

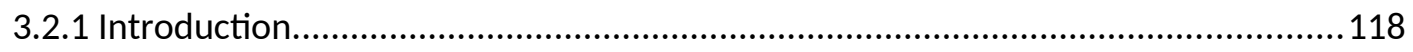

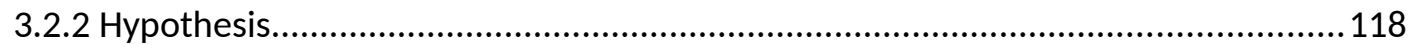

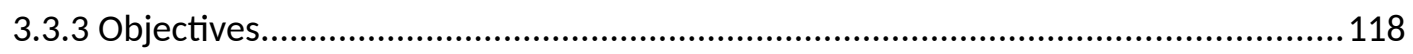

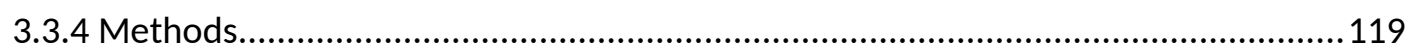

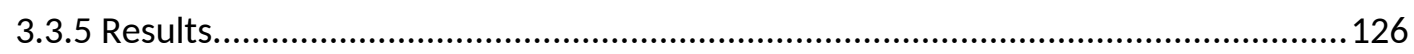

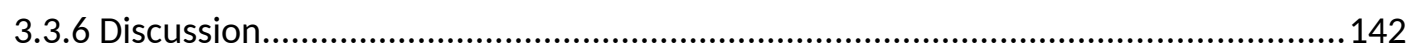

Chapter 4: Severe exacerbations and Type 2 biomarkers in asthma......................146

4.4 Study Five: Change in biomarkers of Type 2 inflammation following severe

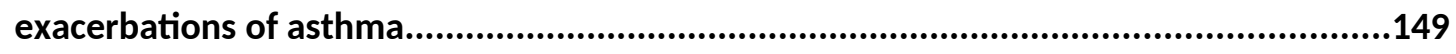

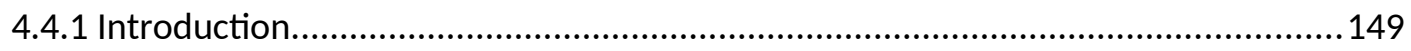

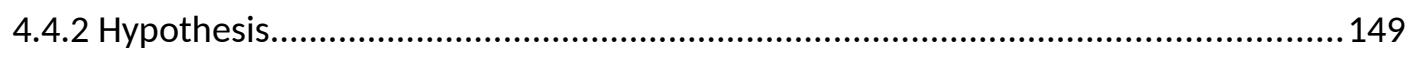

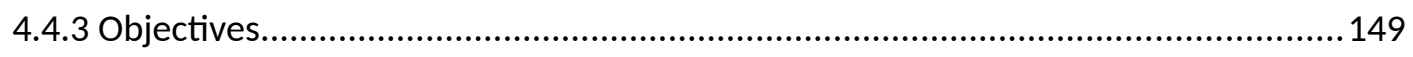

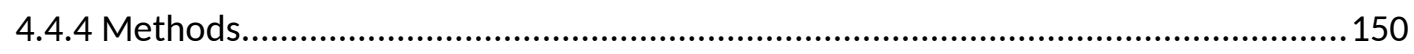




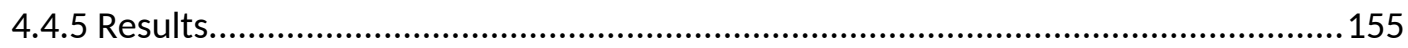

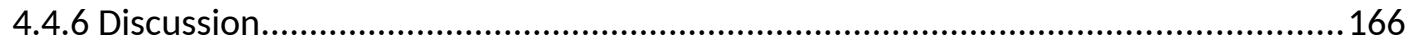

4.5 Study Six: Type 2 biomarkers and prediction of future exacerbations in asthma.......171

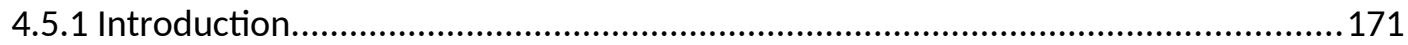

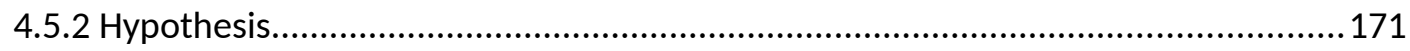

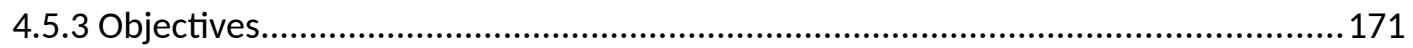

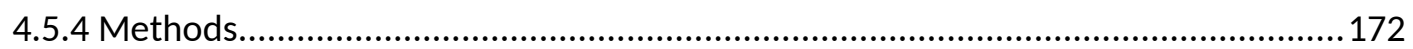

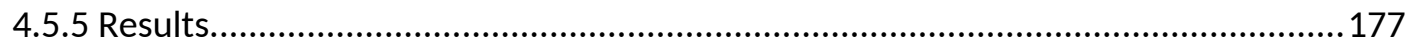

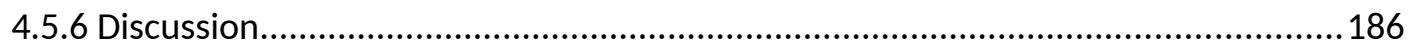

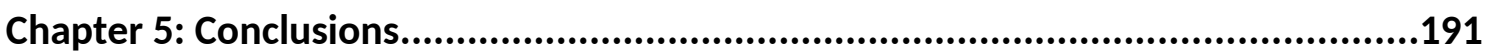

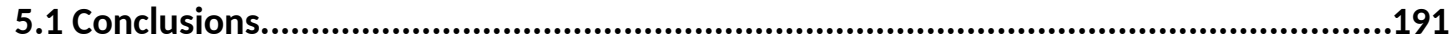

Appendix I: Participant Information Sheet for Study One....................................195

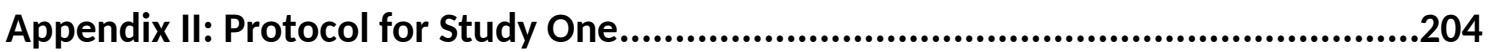

Appendix III: Participant Information Sheet for Study Two................................210

Appendix IV: Protocol for Study Two..............................................................218

Appendix V: Participant Information Sheet for Study Three...............................224

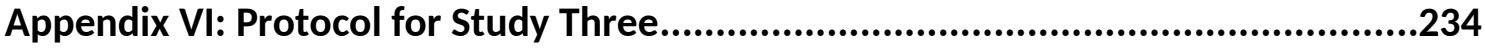

Appendix VII: Participant Information Sheet for Study Four................................248

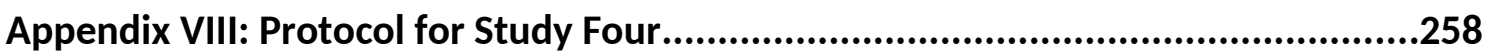

Appendix IX: General Health Questionnaire.................................................271

Appendix X: Asthma Control Questionnaire (ACQ) .............................................278 
Appendix XI: Asthma Quality of Life Questionnaire with Standardised Activities

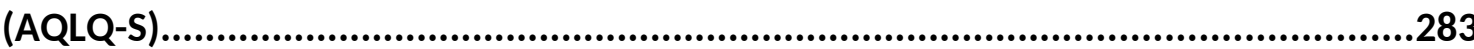

Appendix XII: Participant Information Sheet for Study Five..............................289

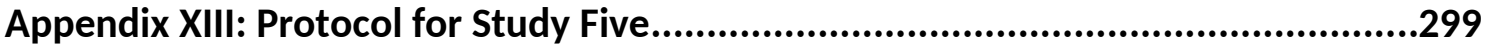

Appendix XIV: Participant Information Sheet for Study Six..................................312

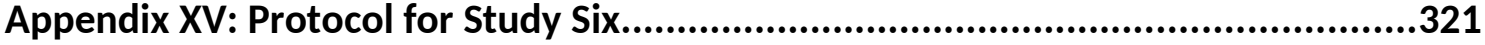

Appendix XVI: Asthma Exacerbation Questionnaire..........................................336

Appendix XVII: Permissions from publications.................................................344 


\section{Publications}

To date, the following manuscripts relating to this thesis have been published.

1. Semprini R, Caswell-Smith R, Fingleton J, Holweg C, Matthews J, Weatherall M, Beasley R, Braithwaite I. Longitudinal variation of serum periostin levels in adults with stable asthma. Journal of Allergy and Clinical Immunology. 2017 May 1;139(5):1687-8. [Study Four, Chapter 3]

2. Varughese R, Semprini R, Munro C, Fingleton J, Holweg C, Weatherall M, Beasley R, Braithwaite I. Serum periostin levels following small bone fractures, long bone fractures and joint replacements: an observational study. Allergy, Asthma \& Clinical Immunology. 2018 Dec;14(1):30. [Study One, Chapter 2]

3. Semprini R, Shortt N, Ebmeier S, Semprini A, Varughese R, Holweg CT, Matthews JG, Fingleton J, Weatherall M, Beasley R, Braithwaite I. Change in biomarkers of type-2 inflammation following severe exacerbations of asthma. Thorax. 2019 Jan, 1;74(1): 95-8. [Study Five, Chapter 4]

4. Semprini R, Williams M, Semprini A, McDouall A, Fingleton J, Holweg C, Weatherall M, Beasley R, Braithwaite I. Type 2 Biomarkers and Prediction of Future Exacerbations and Lung Function Decline in Adult Asthma. The Journal of Allergy and Clinical Immunology: In Practice. 2018 Nov, 1;6(6): 1982-8. [Study Six, Chapter 4]

5. Braithwaite I, Semprini R, Beasley R. The clinical relevance of periostin in asthma. Barcelona Respiratory Network Review. 2018 Oct, 4: 258-73. 
6. Tan E, Varughese R, Semprini R, Montgomery B, Holweg C, Olsson J, Caswell-Smith R, Fingleton J, Weatherall M, Beasley R, Braithwaite I. Serum periostin levels in adults of Chinese descent: an observational study. Allergy, Asthma \& Clinical Immunology. 2018 Dec;14(1):87 [Study 3, Chapter 3]. 


\section{List of Tables}

Table $1.1 \quad$ Key Type 2 cytokines and their function

Table $1.2 \quad$ Potential Type 2 biomarkers

Table 2.1 Baseline participant characteristics for all participants in Study One

Table 2.2 Serum periostin levels at time points in joint replacement group, and ratio of geometric means compared with pre-operative reference baseline value

Table 2.3 Serum periostin levels at time points in long bone fracture group, and ratio of geometric means compared with week 26 reference level

Table 2.4 Serum periostin levels at time points in short bone fracture group, and ratio of geometric means compared with week 26 reference level

Table 2.5 Change in serum periostin over time: differences between the three groups

Table 2.6 Raw serum periostin $(\mathrm{ng} / \mathrm{ml})$ values for Joint Replacement Group

Table 2.7 Raw serum periostin $(\mathrm{ng} / \mathrm{ml})$ values for Long Bone Fracture Group

Table 2.8 Raw periostin (ng/ml) values for Short Bone Fracture Group

Table 2.9 Summary of participant characteristics in Study Two

Table 2.10 Periostin values for the simple and surgical dental extraction groups

Table 2.11 Estimates of change of serum periostin from baseline for both groups

Table 3.1 Participant Characteristics of Non-Asthma groups

Table $3.2 \quad$ Participant Characteristics of Asthma Groups

Table $3.3 \quad$ Study plan for Study Four 
Table 3.4 Participant characteristics for Study Four

Table 3.5 Periostin measurement by participant and day (Visit Day 1 is the baseline measurement)

Table 3.6 Limits of agreement for Periostin (raw values) for a particular visit minus baseline and for adjacent visits

Table 3.7 Least squares mean estimates for Periostin $(\mathrm{ng} / \mathrm{ml})$ by month

Table $3.8 \quad$ Least squares mean estimates for Periostin $(\mathrm{ng} / \mathrm{ml})$ by season

Table 3.9 Cut point analysis of periostin levels (ng/ml), Days 5, 24 and 59 versus baseline

Table 4.1 Study Plan for Study Five

Table $4.2 \quad$ Description of participants at enrolment

Table $4.3 \quad$ Comparison of Type 2 biomarkers with levels at week 12

Table $4.4 \quad$ Comparison of $\mathrm{FEV}_{1} \%$ predicted values with Week 12

Table $4.5 \quad$ Participant characteristics for Study Six

Table 4.6 Association between Type 2 biomarkers and time to severe exacerbation

Table 4.7 Comparison between exacerbators versus non-exacerbators 


\section{List of Figures}

Figure 2.1 Time course of serum periostin levels over 26 weeks in participants who underwent large joint replacements (including pre-operative reference periostin values).

Figure 2.2 Time course of serum periostin levels over 26 weeks in participants who sustained a long bone fracture.

Figure 2.3 Time course of serum periostin levels over 26 weeks in participants who sustained a small bone fracture.

Figure 2.4 Box-plot diagram illustrating serum periostin values by week for those who had a simple tooth extraction

Figure 2.5 Box-plot diagram illustrating serum periostin values by week for those who had a surgical tooth extraction

Figure 3.1 Flow of Chinese participants through the study

Figure 3.2 Histograms illustrating the differences between distribution of serum periostin between Chinese $(n=118)$ and Caucasians $(\mathrm{n}=420)$ without asthma

Figure 3.3 Box-plot of serum periostin by country of birth for both nonasthmatic and asthmatic Chinese participants $(n=185)$

Figure 3.4 Patient flow through Study Four

Figure 3.5 Individual participant mean periostin plus or minus one standard deviation ranked by mean periostin

Figure 3.6 Sequential Bland-Altman plots depicting Limits of Agreement, illustrating between participant variability at consecutive visits compared to baseline

Figure 3.7 Box plot of mean periostin by month

Figure 3.8 Box plot of mean periostin by season

Figure 3.9 Joined line plot by time of the raw periostin levels in the 5 participants that had a severe exacerbation, with prednisone use marked as an event

Figure $4.1 \quad$ Flow of participants through Study Five

Figure 4.2 Boxplot of peripheral blood eosinophils by week after an 
exacerbation

Figure 4.3 Boxplot of Fractional exhaled Nitric Oxide (FeNO) by week after an exacerbation

Figure 4.4 Boxplot of serum periostin by week after an exacerbation

Figure 4.5 Boxplot of serum IgE by week after an exacerbation

Figure 4.6 Flow of participants through Study Six

Figure 4.7 Kaplan-Meier survival curves for quartiles of blood eosinophil count (A), log FeNO (B), log serum periostin (C) and log serum $\operatorname{IgE}(\mathrm{D})$

Figure 4.8 Change in $\mathrm{FEV}_{1}$ with time associated with baseline eosinophil count 


\section{Abbreviations}

$\begin{array}{ll}\text { ATS } & \text { American Thoracic Society } \\ \text { BMI } & \text { Body Mass Index } \\ \text { COPD } & \text { Chronic Obstructive Pulmonary Disease } \\ \text { ERS } & \text { European Respiratory Society } \\ \text { FeNO } & \text { Fractional exhaled Nitric Oxide } \\ \text { FEV }{ }_{1} & \text { Forced Expiratory Volume in one second } \\ \text { FVC } & \text { Forced Vital Capacity } \\ \text { ICS } & \text { Inhaled corticosteroid } \\ \text { IgE } & \text { Immunoglobulin E } \\ \text { IL } & \text { Interleukin } \\ \text { LABA } & \text { Long-acting bronchodilator } \\ \text { MRINZ } & \text { Medical Research Institute of New Zealand } \\ \text { NZRHS } & \text { New Zealand Respiratory Health Survey } \\ \text { PDL } & \text { Periodontal Ligament } \\ \text { SABA } & \text { Short-acting bronchodilator } \\ \text { Th1 } & \text { T-helper cells Type 1 } \\ \text { Th2 } & \text { T-helper cells Type 2 }\end{array}$




\section{Chapter 1: Introduction}

\section{$1.1 \quad$ Asthma}

\subsubsection{What is asthma?}

Asthma is a chronic lung disease that results in inflammation and periodic narrowing of the airways. It is defined by the Global Initiative of Asthma as 'wheeze, shortness of breath, chest tightness and cough that vary over time in their occurrence, frequency and intensity'. ${ }^{1}$ This hyper-reactivity of airways can be triggered by a variety of endogenous and exogenous stimuli such as stress, exercise, tobacco smoke and pollen.

Asthma is a very common chronic health problem, affecting an estimated 300 million people worldwide. ${ }^{1}$ It affects all age groups, ethnicities and socioeconomic groups, with considerable medical and economic cost. ${ }^{2}$ Prevalence of asthma among children and adults has been climbing in recent years, ${ }^{3}$ along with a rise in associated atopic disorders such as eczema and rhinitis. ${ }^{4}$ As a result, there is need for healthcare providers to improve asthma diagnosis and management, to help minimise long term sequelae from this chronic health disorder and ameliorate some of the economic burden of asthma. 


\subsubsection{Pathophysiology of Asthma}

The understanding of the underlying mechanisms of asthma is constantly evolving. Biological heterogeneity exists through the spectrum of asthma but is perhaps most studied in those with severe forms of the disease who have the highest healthcare use with associated morbidity and mortality. Traditionally, those with asthma have been divided by the presence or absence of allergy. Allergic asthma, being the more common form, is triggered by extrinisic allergens such as pollen, dust and household pets and is treated with a combination of bronchodilators (for relief of wheeze) and corticosteroids (for airway inflammation). If symptoms are not adequately controlled, then a step-wise approach to increasing corticosteroid and bronchodilator therapy is recommended ${ }^{1}$. However it is now clear that the 'one size fits all' therapeutic approach does not work within certain subsets of asthmatics. Furthermore, recent randomised controlled trials of biological agents targeting specific aspects of inflammatory pathways have had varying degrees of success in heterogeneous asthma populations, suggesting that current understanding of the pathophysiology of asthma is far from clear.

Asthma was once thought to be driven by the adaptive immune system. In 1986, an immunological breakthrough was made when two discrete groups of T-helper cells, which secreted different cytokine profiles, were described - T helper 1 (Th1) and T helper 2 (Th2) cells. ${ }^{5}$ Th1 cells secrete mainly interleukin (IL)-2, interferon (IFN)- $\Upsilon$ and lymphotoxin- $a,{ }^{6}$ which activate against viral and bacterial pathogens that target intracellular mechanisms. Th2 cells secrete IL-4, IL-5, IL-9 and IL-13 which are most 
effective against bacteria targeting extracellular mechanisms or helminths and are found in pathological levels in asthma. ${ }^{6,7}$ By the early 1990s, mouse models showed that activation of Th2 cells resulted in mast cell activation and eosinophilic airway inflammation ${ }^{8-10}$ causing asthma-like changes in the airways. This was supported by clinical studies in humans which demonstrated large numbers of Th2 cells in bronchial fluid from individuals with atopic asthma, ${ }^{11}$ leading to the assertion that asthma was primarily a disease of allergy, driven by the Type 2 inflammatory pathway.

The Type 2 inflammatory pathway is initiated by IL-25, IL-33 and Thymic Stromal Lymphopoeietin (TSLP), epithelial cell derived cytokines, which are released in response to an airway insult. They activate antigen presenting cells (APCs), which in turn induce the Type 2 adaptive immune response. Naïve T-cells are activated, and differentiate into either Th1 or Th2 cells, depending on the mix of local cytokines and doses of antigen being presented. ${ }^{12,13} \mathrm{IL}-4$ is integral in tipping the balance toward the Type 2 pathway. ${ }^{13,14}$ Activated Th2 cells migrate to the airway epithelium and stimulate the release of IL-5 and IL-13 which signal a cascade of events resulting in increased mucous production, IgE synthesis, subepithelial fibrosis, bronchial remodelling and airway hyper-responsiveness (AHR), ${ }^{15,16}$ key characteristics of asthma.

These Type 2 cytokines drive the recruitment of mast cells, basophils and eosinophils whilst mediating a switch of B-cell secreted immunoglobulins to IgE (Table 1.1). ${ }^{7}$ Therefore eosinophils and IgE are often synonymous with Type 2 asthma. 
Table 1.1: Key Type 2 cytokines and their functions

\begin{tabular}{|c|c|}
\hline Cytokine & Function \\
\hline IL-4 & $\begin{array}{l}\text { Binds to IL- } 4 \text { receptor- } \alpha \text { (IL- } 4 \mathrm{R} \alpha),{ }^{17} \text { which is widely } \\
\text { expressed on T-helper cells, eosinophils, mast cells, B cells, } \\
\text { bronchial epithelium, endothelium and airway smooth } \\
\text { muscle cells. Activation of IL- } 4 \mathrm{R} \alpha \text { results in either a Type } 1 \\
\text { or a Type } 2 \text { signal. A Type } 1 \text { signal modulates T-helper cell } \\
\text { differentiation. A Type } 2 \text { signal activates release of IL-13, a } \\
\text { potent effector cytokine. }{ }^{18}\end{array}$ \\
\hline IL-13 & $\begin{array}{l}\text { Stimulates B cell isotype switching, mucus hypersecretion, } \\
\text { goblet cell hyperplasia, subepithelial fibrosis and AHR, }{ }^{19,20} \\
\text { through cholinergic-induced contraction of smooth } \\
\text { muscle }{ }^{21,22} \text {, as well as the recruitment of eosinophils. }{ }^{19,23} \mathrm{IL}- \\
13 \text { receptors are expressed on airway smooth muscle cells } \\
\text { and airway epithelial cells. }\end{array}$ \\
\hline IL-5 & 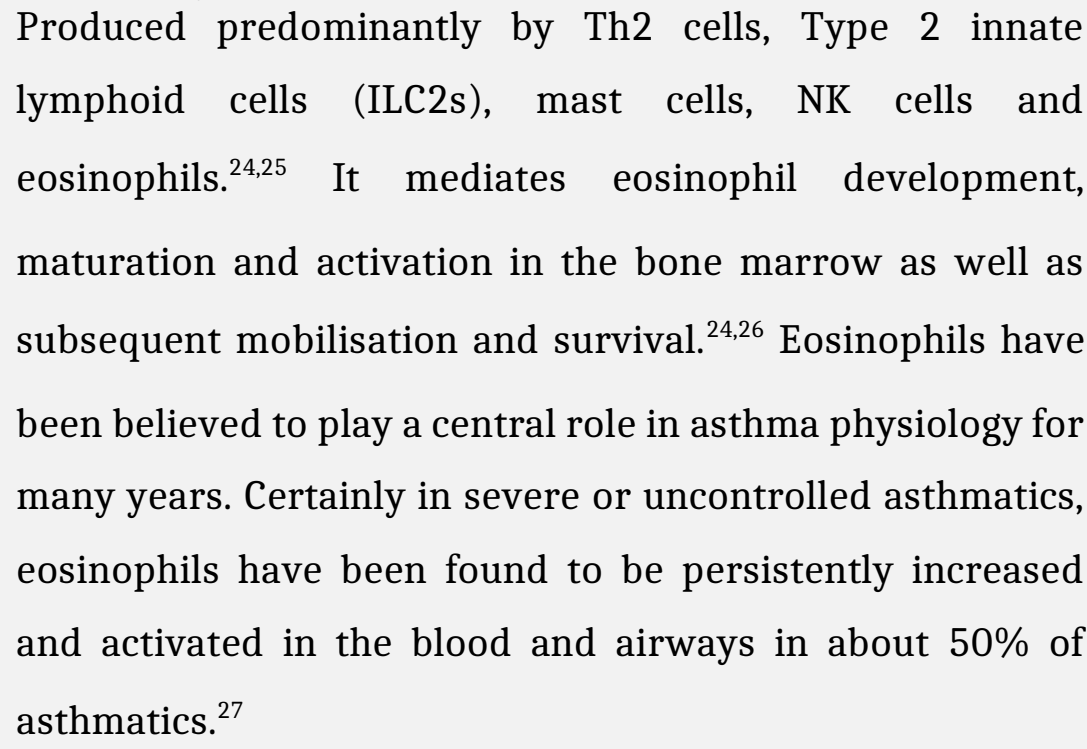 \\
\hline IL-9 & $\begin{array}{l}\text { Secreted by mast cells, Th2 cells and ILC2 cells. It stimulates } \\
\text { proliferation of activated T-cells and mast cells. }{ }^{17,28} \text { It also } \\
\text { induces IgE production from B cells. In human studies, IL-9 } \\
\text { increases pre-disposition to AHR as well as contributing to } \\
\text { mucus hypersecretion. }{ }^{17}\end{array}$ \\
\hline
\end{tabular}


However, this dichotomous division of asthma classification into allergic/non-allergic asthma has been shown to be an over-simplification with several research groups describing the involvement of innate immunity in the development of airway inflammation found in asthma, ${ }^{28,29}$ and randomised controlled trials demonstrating differential response to asthma treatments based on the type of underlying airway inflammation..$^{30,31}$ There is now a growing body of evidence to suggest that asthma is not a single disease, but rather a spectrum of disorders, with multiple sub-groups or phenotypes, with differing patterns of airway inflammation which are likely to respond to treatment differently.

\subsubsection{Causes of Asthma}

Asthma causality is not fully understood though a number of factors are considered to increase one's risk for developing the condition. ${ }^{32}$ Genetic predisposition is undoubtedly a factor, though no specific gene has been found to cause asthma. Family history, gender and intra-uterine exposures are all thought to contribute to asthma risk..$^{33}$ The growing study of epigenetics, the study of gene expression changes that are not as a result of altered gene sequence, has led to associations between microbial exposure, dietary influences, obesity, tobacco smoke and pollutant exposure and the risk of developing asthma. ${ }^{32}$ This theory is supported by evidence that children with asthma demonstrate dysregulated immune pathways, contributing to uncontrolled Type 2 immunity, ${ }^{34,35}$ which is detectable at birth. ${ }^{36}$ 
Childhood exposures to viruses, such as the respiratory syncytial virus and rhinovirus, are associated with the development of asthma, ${ }^{37}$ particularly if the infant has concurrent atopy. Although conversely, attendance at day-care or the presence of more than one sibling at home confers protection against the development of asthma, despite increased episodes of viral induced wheeze. ${ }^{38}$

Medication use can also increase an individual's risk of asthma. Over-use of beta agonists has been shown to increase AHR in both children and adults ${ }^{39,40}$ which in turn can lead to the development of severe asthma. Paracetamol use has also been linked to asthma aetiology, ${ }^{41}$ however has not been proven unequivocally. Allergen exposure and asthma prevalence do not have a linear relationship in terms of causation ${ }^{42}$ and differ between populations and the degree of urbanisation, suggesting a more complex genetic/environmental interplay, which increases risk of developing disease.

Current understanding of what causes asthma has come from either animal studies, which are not translatable into human subjects or epidemiological studies, which are limited by their methodology and vulnerability to systemic bias. Given the heterogeneity of asthma, it is likely that causation is multi-faceted with a combination of genetic and environmental factors. 


\subsubsection{Asthma Classification}

Asthma was originally described by Hippocrates in $450 \mathrm{BC}$ as a single disease state. It is now recognised to be a heterogeneous disorder with variable severity, natural history and response to treatment. ${ }^{43}$ Asthma's heterogeneity can range from mild to severe and patients may demonstrate intermittent or persistent bronchospasm, making classification challenging because of the lack of specific and validated markers. Traditionally asthmatics were categorised as atopic/extrinsic or non-atopic/intrinsic, both characterised by eosinophilic inflammation. ${ }^{44}$ Most children and up to $50 \%$ of adults suffer with atopic asthma. ${ }^{45}$ It is characterised with childhood allergic sensitisation to common allergens and there is usually a positive family history. Atopic asthma tends to be more responsive to treatment with inhaled corticosteroids (ICS). Features of non-atopic asthma include later onset of disease, with no involvement of the allergic immune system. It tends to be associated with other co-morbidities such as nasal polyps, obesity and chronic rhinosinusitis. ${ }^{45}$ Non-atopic asthma is also more difficult to treat, and can be less responsive to corticosteroid therapy. ${ }^{46}$ However it has become clear that this is an oversimplification of asthma and since then further classification systems have been suggested.

Currently asthmatics are treated according to the severity of their symptoms, with medication increased until their symptoms are no longer 'problematic'. However, this strategy does not take into account the heterogeneity of asthma, or the possibility that 
symptom control and underlying airway inflammation control may not be one and the same thing.

With $5-10 \%$ of asthmatics refractory to conventional treatment, ${ }^{45}$ research into phenotype-based definitions of asthma has increased over recent years to aid treatment decisions. Phenotype-based descriptions of asthma are reflective of clinical and morphologic characteristics as well as response to treatment. Phenotyping in asthma has been used to discriminate between differences in symptoms, severity and response to treatment. Clinical characteristics such as age, gender, time of onset, smoking and environmental exposures and associated co-morbidities have all come into play when describing various phenotypes. Large epidemiological programmes have been started in recent years, attempting to describe and classify asthma spectrum disorders. Disadvantages of phenotype-based descriptions are that they do not describe the underlying pathological mechanism ${ }^{47}$ and can change with time or in response to treatment. ${ }^{48}$ Consequently, endotype-based definitions of asthma are emerging, which sub-divide asthmatics based on their cellular and molecular pathology ${ }^{49}$ in the hope that this will enable clinicians to tailor treatments based on the underlying pathology of an individual's disease.

Type 2 asthma

Type 2 asthma is the best defined endotype to date. Asthmatics categorised based on their Type 2 immunity status, using concentrations of IL-4, IL-5 and IL-13 in blood and tissue, were labelled as Type 2 'high' or Type 2 'low'. ${ }^{50,51}$ Importantly, Type 2 'high' 
asthmatics were more responsive to corticosteroid therapy than their Type 2 'low' counterparts, irrespective of asthma severity.

The strongest evidence for Type 2 asthma comes from clinical trials testing monoclonal antibody therapy directed against components of the Type 2 inflammatory pathway. The monoclonal antibody targetting IL-4Ra, thereby blocking downstream signalling of IL-4, improves lung function and exacerbation rates in people with moderate to severe asthma. ${ }^{52}$ Monoclonal antibody agents directed against IL-13 also improve lung function in severe asthmatics. ${ }^{53,54}$ The inhibition of IL-5, resulted in improved asthma control, fewer exacerbations and improved lung function in severe eosinophilic asthmatics. ${ }^{55}$ Finally, biological agents directed against immunoglobulin E (IgE) have also been found to be effective in improving asthma control and reducing exacerbations in moderate to severe, poorly controlled allergic asthmatics. ${ }^{56}$ In all these clinical trials, the biological agents were most effective in pre-defined Type 2 'high' populations of asthmatics, highlighting the need of being able to identify responders before initiating biological therapy. There is an inherent difficulty and expense in measuring Type 2 cytokines in vivo, therefore there is a need for surrogate biomarkers, such as blood eosinophils, Fractional exhaled Nitric Oxide (FeNO), serum periostin and serum IgE. These biomarkers have several potential roles in asthma management: identifying those with Type 2 asthma who are most likely to respond to targeted biological therapies, monitoring response whilst on these therapies and predicting prognosis of asthma. 
Non-Type 2 asthma

Little is understood about non-Type 2 asthma. Asthmatics in this endotype display less airway obstruction and hyper-reactivity ${ }^{51}$, have no family history ${ }^{57,58}$ and are less likely to respond to corticosteroid therapy. It is likely driven by an alternative inflammatory pathway (or pathways) to Type 2 asthma, with sufferers demonstrating different inflammatory footprints to their Type 2 counterparts. An increasingly popular theory behind non-Type 2 asthma is the role of the innate immune system, with higher expression of IL-8, IL-17, IL-2, INF- $\Upsilon$ and mRNA expression of several innate immune response receptors. ${ }^{59-61}$ Asthmatics with neutrophil-dominant airway inflammation have the highest prevalence in groups with the severest forms of the disease whilst being amongst the most resistant to treatment. ${ }^{62}$ Asthmatic airways have also been found to have high levels of intracellular bacteria within neutrophils and macrophages, ${ }^{59}$ which may explain why macrolide antibiotics have been effective in lowering exacerbation rates and improving quality of life in asthmatics with low blood eosinophil counts. ${ }^{63}$

The limited understanding behind non-Type 2 asthma pathways has meant there has been little progress in developing therapeutic agents. However, large scale longitudinal studies are underway, focused on the stratification of asthma and potential surrogate biomarker use, ${ }^{64,65}$ which will hopefully provide deeper insight into asthma endotypes and clinically viable means of distinguishing them. 


\subsubsection{Current therapeutic options in asthma}

Inhaled corticosteroids (ICS), in combination with short- or long-acting $\beta$-agonists where necessary, are the mainstay of asthma treatment. Corticosteroids have a broad and non-specific mode of action ${ }^{66,67}$ and serve in modulating T2 cytokines. However, 5$10 \%$ of asthmatics remain poorly controlled despite being on maximal doses of corticosteroids. Frequent or long-term use of systemic corticosteroids come with significant health consequences. Therefore novel biological therapies targeting specific inflammatory cascades are being developed in order to provide more specific treatment options for patients requiring high doses of corticosteroid to achieve adequate asthma control.

However, as previously mentioned, most biologics to date target the Type 2 inflammatory pathway. There are currently two biological agents approved for add on therapy in asthma in New Zealand, when conventional treatment is insufficient omalizumab (a humanised monoclonal antibody directed against circulating IgE) and mepolizumab (monoclonal antibody directed against IL-5). Both have been shown to reduce exacerbations and improve asthma control in pre-defined populations. ${ }^{55,56}$ Omalizumab is currently only licensed for use in the UK for severe asthmatics with proven allergic asthma (either by skin prick test or total serum IgE of greater than $300 \mathrm{U} / \mathrm{L}$ ), with a history of multiple exacerbations requiring the use of systemic corticosteroids despite being on maximal inhaled treatment. ${ }^{68}$ Subsequent clinical trials also showed that asthmatics with a peripheral blood eosinophil count of greater than $0.26 \times 10^{\circ} / \mathrm{L}$ had fewer exacerbations and better lung function after treatment with 
omalizumab, ${ }^{69}$ suggesting that blood eosinophil count may also be a predictor biomarker for this therapeutic agent, though this is not a criterion for prescription of omalizumab. Mepolizumab was recently approved for use in the UK in January 2017 for those with severe eosinophilic asthma, with documented peripheral blood eosinophil levels of greater than $0.3 \times 10^{\%} / \mathrm{L}$ and who suffer with recurrent exacerbations requiring systemic corticosteroid, despite being on maximal inhaled treatments. ${ }^{70}$ More recently, two more monoclonal antibodies have been given approval for use in eosinophilc asthmatics in the USA, UK and Europe as second line agents: benralizumab and reslizumab. Both agents target IL-5 and have been shown to reduced exacerbation rates by approximately $50 \%$ when used in poorly controlled asthmatics, with blood eosinophils greater than $0.3 \times 10^{9} /$ L. $^{71,72}$

Several molecules are in various forms of development. Dupilumab, an IL-4Ra blocker, has been shown to improve exacerbation rates in moderate to severe asthmatics with high blood eosinophil counts and high periostin levels. ${ }^{52}$ Whilst anti-IL-13 agents such as lebrikizumab ${ }^{53}$ and tralokinumab ${ }^{54}$ have both been shown to improve lung function and reduce exacerbation rates in severe asthmatics who demonstrate high pretreatment periostin levels.

Data from these clinical studies indicate targeted asthma therapy can be provided to patients who do not respond to corticosteroid treatment, provided they can be identified using validated surrogate biomarkers, which accurately reflect the underlying pathophysiological mechanism of their asthma. Potential surrogate 
biomarkers include: peripheral blood eosinophils, FeNO, serum periostin and serum $\lg \mathrm{E}$. 


\subsection{Biomarkers}

\subsubsection{What is a biomarker?}

A biomarker is a surrogate measurement which can characterise or quantify an underlying disease process. ${ }^{73}$ The National Institutes of Health Biomarkers Definitions Working Group define a biomarker as a 'characteristic that is objectively measured and evaluated as an indicator of normal biological processes, pathogenic processes or pharmacologic responses to a therapeutic intervention' ${ }^{74}$ The success of a biomarker in clinical practice is dependent on the question being asked, and the properties of the designated marker. A biomarker can be used to either rule out a disease process or suggest activity of a disease. For example, in those with moderate to high risk of venous thromboembolism, a d-dimer blood test has a high negative predictive value for the absence of a clot. ${ }^{75}$ Whereas, prostate specific antigen (PSA) has a high positive predictive value for prostate cancer if levels are above a cut-point of $4 \mathrm{ng} / \mathrm{ml} .{ }^{76}$

Biomarkers can be used to risk stratify a general population, screen for underlying disease, diagnose or stage active pathology, predict prognosis or monitor disease activity, which enable clinicians to modify treatment regimens. A good biomarker should be minimally invasive, easily measurable and reproducible. ${ }^{73}$ To be used clinically, rigorous trialling and validation should take place to ensure uniform interpretation of biomarker values. 


\subsubsection{The role of biomarkers in Asthma}

Biomarkers in respiratory disease have a role in monitoring disease activity or response to treatment. Surrogate markers are all the more important given logistical difficulties of obtaining samples directly from the respiratory system. The diagnosis of asthma is often based on symptoms and clinical findings. Around $50 \%$ of asthmatics will demonstrate eosinophilic airway inflammation, for which the 'gold standard' diagnostic test is a bronchial sampling or sputum induction. However, these tests require specialist facilities and have inherent procedural risk for patients.

Asthma is conventionally treated empirically based on stepwise treatment with short acting bronchodilators, followed by ICS and further add on therapy until adequate asthma control is achieved. ${ }^{1}$ Response to treatment is usually measured indirectly through symptom score questionnaires, basic lung function measurements and exacerbation rates, which does not necessarily reflect the activity of underlying airway inflammation. Surrogate biomarkers, which accurately reflect this airway inflammation could not only result in the need for lower doses of corticosteroids, but facilitate the use of biological agents which target specific components of the inflammatory pathway driving asthma pathogenesis.

Currently in asthma the most researched endotype is Type 2 inflammation, for which there are several potential biomarkes, denoted in Table 1.2. The four main biomarkers which have been examined in this thesis are: peripheral blood eosinophils, FeNO, serum IgE and serum periostin. All of these biomarkers are measureable peripherally 
and are more easily obtainable than direct bronchial sampling. They have each been validated to different points and may have an individual or combined role in facilitating sub-classification of Type 2 asthma.

Table 1.2 Potential Type 2 biomarkers

\begin{tabular}{llll}
\hline Biomarker & Testing method & Phenotype & Associated cytokines \\
\hline Eosinophils & Blood, sputum & Allergic, & IL-5, IL-4 \\
& & eosinophilic & \\
Neutrophils & Blood, sputum & Non-allergic, & IL-8, IL-17 \\
& & neutrophilic & \\
IgE & Blood & Allergic & IL-4, IL-13 \\
Periostin & Blood, sputum & Eosinophilic & IL-4, IL-13 \\
DPP-4 ${ }^{1}$ & Blood & Eosinophilic, & IL-13 \\
& & AERD & \\
& &
\end{tabular}

1. DPP-4: dipepidyl-dipeptidase-4

2. AERD: aspirin-exacerbated respiratory disease

\subsubsection{Peripheral blood eosinophils}

Eosinophils were first associated with asthma in $1908^{77}$ and have been extensively studied as a potential biomarker for asthma. Blood eosinophils are important mediators of inflammation as, once activated, they release toxic granule proteins and pro-inflammatory cytokines, which result in tissue damage and dysfunction. ${ }^{78}$ In 
airways, eosinophils mediate remodelling and smooth muscle hypertrophy, resulting in chronic airflow obstruction in asthma. High levels of peripheral eosinophils are associated with higher symptoms scores, lower $\mathrm{FEV}_{1}$ values and greater diurnal peak expiratory peak flow variation, characteristics which are associated with a raised risk of severe exacerbations in asthma. ${ }^{79}$ The monoclonal antibody, mepolizumab, directed against IL-5 can suppress blood and sputum eosinophils by up to $80 \%^{80}$ and reduce frequency of exacerbations in patients with uncontrolled eosinophilic asthma, who are on high dose corticotseroids. ${ }^{81}$ These effects are greater in patients who have pretreatment blood eosinophil levels of greater than $0.15 \times 10^{9} / L .{ }^{55}$ Peripheral blood eosinophils are predictive of response to other monoclonal antibody therapies directed against $\lg \mathrm{E}^{69}$ and $\mathrm{IL}-13^{53}$, though with differing pre-treatment cut-offs (greater than $0.26 \times 10^{9} / \mathrm{L}$ and $0.14 \times 10^{9} / \mathrm{L}$, respectively). Taken together, these findings suggest that peripheral blood eosinophils have a role in predicting asthma severity, likelihood of severe exacerbation and potential response to treatment with biological agents.

Whilst the measurement of blood eosinophils is a readily available and inexpensive test, there are disadvantages to its use. Firstly, peripheral eosinophils may be elevated due to non-asthma reasons, such as parasite infections, autoimmune diseases and allergies. Secondly, as eosinophils are primarily tissue dwelling leucocytes, blood eosinophils may not accurately reflect bronchial activity, ${ }^{82}$ particularly in cases where the patient has taken ICS or anti-IL-5 therapy. ${ }^{80,83}$ In addition, blood eosinophils have a short circulating half-life (of around 18 hours), making them highly variable within an individual. Despite these issues, peripheral blood eosinophils may have a role in the diagnosis and management of asthma, particularly if used to select patients for 
monoclonal antibody therapy directed against IL-5, IL-4R $\alpha$, IL-13 or IgE. However, its use as a biomarker of disease activity may be limited once the patient is on treatment.

\subsubsection{FeNO}

FeNO correlates with eosinophilic airway inflammation, when compared to eosinophils present in samples from bronchial washings,${ }^{84}$ bronchial biopsies ${ }^{85}$ and induced sputum samples. ${ }^{86-88}$ A standardised method for obtaining FeNO has been outlined in international guidelines, ${ }^{89}$ ensuring that it is a safe, reproducible biomarker for asthma. Raised FeNO (greater than 47ppb) is suggestive of airway eosinophilia as well as being a predictor of response to corticosteroid therapy ${ }^{82}$ and risk of exacerbations. ${ }^{90}$ Similarly, those with high FeNO measurements benefit more from treatment with a monoclonal antibody directed against $\lg ^{69}$ whilst those with a consistently low FeNO (less than $25 \mathrm{ppb}$ ) may be less responsive to treatment with ICS. ${ }^{91}$ Although FeNO can be elevated in non-asthmatics, it is generally higher within an asthmatic population than a non-asthmatic population. ${ }^{82}$

FeNO levels can be affected by factors other than asthma such as age, gender, smoking status and medication use. ${ }^{83}$ It does not correlate with asthma severity. ${ }^{92}$ Whilst it is reflective of eosinophilic airway inflammation, it is not a duplicate measure as evidenced by the fact that sputum eosinophilia can be reduced by anti-IL- 5 therapy without affecting FeNO levels. ${ }^{81}$ It is also highly variable within an individual, changing up to $20 \%$ within a patient with stable asthma (and no change to treatment) over two 
weeks. ${ }^{53}$ In all, the role of FeNO in asthma may lie with its diagnostic capabilities as well as identifying those who are most likely to respond to corticosteroid therapy rather than predicting potential responders to monoclonal antibody therapy.

\subsubsection{Serum IgE}

Total serum IgE is an important marker of atopy, ${ }^{82}$ which in itself is an important asthma phenotype. The presence of allergen-specific IgE is considered diagnostic for allergic asthma ${ }^{82}$ and total IgE can be used to characterise a population for targeted intervention. IgE can stimulate the release of pro-inflammatory mediators through activation of the allergy cascade. High levels of lung parenchymal IgE is positively correlated with a history of asthma-related ventilation, ${ }^{93}$ whilst elevated levels of serum IgE are associated with an increased exacerbation rate. ${ }^{94}$ Furthermore, monoclonal antibody therapy directed against IgE improves asthma control and reduces exacerbations by $25 \% .{ }^{56}$

The major disadvantage of serum IgE is the large overlap between atopic and nonatopic populations, meaning that it is not useful in the diagnosis of asthma. Other factors also affect serum levels, such as age and gender. ${ }^{95}$ Additionally, total IgE levels are not predictive of response to the anti-IgE monoclonal antibody, omalizumab. ${ }^{96}$ Therefore, its role as a biomarker is likely to lie in characterising asthma and predicting outcomes in terms of exacerbations. 


\subsubsection{Serum periostin}

The periostin gene (POSTN) was found to be highly expressed in a group of asthmatics who demonstrated Th2 high asthma ${ }^{51}$ and is induced by IL-4 and IL-13 in airway epithelial cells and lung fibroblasts. ${ }^{16}$ The periostin protein is involved with airway remodelling and subepithelial fibrosis ${ }^{16}$ and elevated levels are associated with a higher exacerbation rate and greater decline in lung function over several years. ${ }^{97}$ Serum periostin levels predict airway eosinophilia in poorly controlled asthmatics on maximal corticosteroid therapy, ${ }^{98}$ although this finding has not be reproduced. ${ }^{99}$ Monoclonal antibody therapy directed against components of the Type 2 inflammatory pathway is more efficacious in those with high serum periostin levels. ${ }^{52-54,69}$

A major disadvantage to the utility of serum periostin as a biomarker in asthma is that it cannot distinguish between asthmatic and non-asthmatic populations, ${ }^{100,101}$ nor can it usefully discriminate between severity in those with asthma. ${ }^{100}$ It is a ubiquitous matricellular protein with functions in multiple organ systems and therefore pathology within these systems could have an effect on serum periostin levels, although the full extent of this has not been investigated. Finally, it is not clear from current evidence if the reference ranges described for serum periostin need modification for different ethnic groups, as in the case with serum IgE. ${ }^{102}$ In summary, serum periostin may be a useful biomarker in identifying Type 2 asthma and those who would respond to biological agents directed against the Type 2 pathway, but it would not be useful in the diagnosis of asthma. 


\subsection{Periostin}

\subsubsection{What is periostin?}

Originally termed osteoblast-specific factor 2 (OSF-2), periostin is a 93.3kDa, vitamin Kdependent glutamate containing matricellular protein. ${ }^{103}$ It was first identified in 1993 through subtraction hybridisation of a mouse embryonal cell line MC3T3-E1. ${ }^{104,105}$ In humans, it is encoded by the POSTN gene, located on chromosome 13 (13q, 13.3). ${ }^{106}$ Periostin expression is induced by a wide variety of factors in a cell-specific context, including Tissue Growth Factor- $\beta$ (TGF- $\beta$ )-1, -2 and -3 , Bone Morphogenic Protein (BMP)-2 and -4, vascular endothelial growth factor, connective tissue growth factor-2, vitamin $\mathrm{K}$, valsartan (angiotensin II antagonist) and IL-3, 4, 6 and $13 .{ }^{107}$

Periostin was initially thought to be a simple adhesion molecule, binding several integrins on its cell surface. However, it has since been assigned to the fascicilin family of matricellular proteins as it has four repeat domains that resemble FAS1, which allow binding of integrins and glucosaminoglycans. ${ }^{108}$ It also consists of a cysteine-rich $\mathrm{N}$ terminus, about 75 amino acids long, with an EMI domain, and an alternative-splicing region at its C-terminal. ${ }^{105}$

As a matricellular protein, periostin can interact with av-integrins to induce downstream signalling pathways which modulate expression of multiple genes including a-smooth muscle actin, collagen, fibronectin, aggrecan, sclerostin, 
chemokines and TGF- $\beta 1 .{ }^{109-111}$ It has defined functions in bone injury, tissue repair, dental health, cancer, as well as cardiovascular, renal and respiratory diseases. Interest in periostin has increased after emergence of data associated it with Type 2 inflammation in asthma. ${ }^{50,51}$ Serum periostin levels have been found to be higher in children, ${ }^{16}$ which is perhaps unsurprising as it is upregulated in times of growth and remodelling. However, its usefulness in children with asthma has yet to be determined. ${ }^{112}$ Several isomers have been described, ${ }^{107,113,114}$ although their functional implications are not fully understood. There is currently only a single clinical grade assay, Elecsys ${ }^{\circledR}$ Periostin Immunoassay, which uses two monoclonal antibodies to measure periostin isomers, ${ }^{115}$ though the details of this assay are presently protected under patent. Other assays, measuring periostin isomers through ELISA techniques also exist, but it is not known how these assays would compare to the Elecsys ${ }^{\circledR}$ Periostin Immunoassay as there have been no head-to-head trials.

\subsubsection{Periostin in bone injury}

Periostin was renamed in 1999 after elevated levels were found within the periosteum, the layer of connective tissue surrounding the bone. ${ }^{104}$ The periosteum plays a central role in the process of fracture repair. Periostin's high levels of expression within the periosteum may be explained by its likely role in bone's structural integrity and strength. 
In vitro, periostin stimulates osteoblast proliferation, differentiation, adhesion and prolonged survival. ${ }^{116}$ Inactivation of the POSTN gene in mouse models result in lower levels of osteoblast-specific factors, including alkaline phosphatase (ALP), osteopontin, Type 1 collagen, osteocalcin and disorganised collagen matrix formation. ${ }^{110,117}$ Consequently periostin-null mice demonstrate marked growth retardation and shortened long bones. In addition, there is an increase in expression of sclerostin mRNA resulting in sclerostin-mediated bone loss. ${ }^{110}$ Taken together, this suggests that periostin plays a vital role in bone strength and mechanics.

In the neonate and during embryogenesis, periostin is highly expressed, presumably for bone development and maturation. ${ }^{118}$ There is down-regulation of periostin in the post-natal period although it is re-expressed during fracture repair or in times of mechanical stress. ${ }^{119}$ Periostin mediates its effects on bone remodelling by regulating collagen cross-linking and fibrillogenesis by binding to BMP-1 via the EMI domain. ${ }^{120}$

\subsubsection{Periostin in tissue repair}

Tissue repair after injury results in increased periostin expression transiently, peaking at seven days before returning to baseline 28 days later. ${ }^{121}$ In conditions of chronic tissue inflammation and fibrosis, periostin-like factor was identified in satellite cells. ${ }^{122}$ In healthy human skin, periostin is expressed at basal levels but then localises to the extracellular compartment during tissue remodelling. ${ }^{123}$ Periostin is thought to promote healing by facilitating the activation, differentiation and contraction of 
fibroblasts. ${ }^{121,124,125}$ These observations support the hypothesis that periostin has a role in tissue repair and regeneration.

\subsubsection{Periostin in dental health}

The periodontal ligament (PDL) is one of the structures that connect the teeth to the jaw and help them withstand the pressures of mastication. It is an important structure for the maintenance of healthy teeth and bone. The PDL responds to mechanical stress by remodelling. ${ }^{103}$ Periostin is highly expressed in the PDL of murine models ${ }^{104}$ and, in periostin-null mice, there is disorganised structure and mineralisation of murine teeth as well as a weaker PDL. ${ }^{113}$ As tendons are key in transmitting forces of contraction from muscle to bone, disruption to collagen organisation interferes with effective transfer of forces, resulting in poorer dental health. This has been shown in periostinnull mice, which demonstrate abnormal remodelling of the PDL, destruction of the alveolar bone (presumably from abnormal loading), enamel defects in teeth and a periodontitis-like disorder, characterised by abnormal tooth migration and premature tooth loss. ${ }^{117}$

\subsubsection{Periostin in Cancer}

Periostin is over expressed in a wide variety of cancer ${ }^{126}$ which include, thymoma, nonsmall cell lung cancer, breast cancer, pancreatic ductal adenocarcinoma and ovarian cancer. In solid tumour cell lines, it has been shown to promote tumour angiogenesis, 
migration and metastases. ${ }^{127}$ These findings have been supported by clinical studies that show metastatic disease is associated with increased periostin levels. ${ }^{127-130}$

Periostin is thought to stimulate cancer growth by preventing cell apoptosis and improving the tumour's microenvironment to encourage growth, survival and invasiveness of the cancer. ${ }^{131}$ The underlying mechanism of this is the induction of expression of MMP-9, MMP-10 and MMP-13, which result in the degradation of the extracellular membrane resulting in tumour growth spread. ${ }^{132,133}$

\subsubsection{Periostin and the Cardiovascular System}

In utero, periostin plays a vital role in the differentiation and development of heart valves and the fibrous cardiac skeleton. ${ }^{103}$ Expression of periostin induces collagen production, compaction and fibroblast proliferation, resulting in ventricular wall strength and valve maturation. ${ }^{109}$ Periostin null mice have truncated heart valves with ectopic cardiomyocytes, predisposing the mouse to certain forms of arrhythmia. ${ }^{134}$ As with bone and the PDL, periostin is downregulated in the postnatal period, and only reexpressed after injury. Postnally, periostin is found in cardiac fibroblasts and myofibroblasts at a low level of expression. In times of cardiac stress, periostin levels increase and cause accelerated mobilisation, tissue engraftment and differentiation of bone marrow cells into cardiac fibroblasts, ${ }^{135}$ enabling rapid tissue remodelling and fibrosis. This is supported by murine model studies where periostin-null mice are more prone to ventricular rupture ten days after a myocardial infarction. ${ }^{114}$ There is 
mounting evidence that periostin insufficiency is associated with valvular heart disease, ${ }^{107,136}$ heart failure ${ }^{137,138}$ and atherosclerosis. ${ }^{139}$ Thus the POSTN gene is a potential target for the prevention or treatment of heart failure. ${ }^{137,138}$

\subsubsection{Periostin and Renal Disease}

Periostin is induced during nephrogeneis by BMP-4. ${ }^{140}$ It is highly expressed in mesenchymal tissue surrounding the kidney and ureters as well as in the renal stroma. ${ }^{141,142}$ As with other tissues, periostin is down regulated postnatally until renal insult or injury, where it is re-expressed within renal tubules ${ }^{141}$ and blood vessels. ${ }^{143}$ It is particularly over-expressed in hypertensive nephropathy, ${ }^{143}$ making it a potential biomarker for this condition.

\subsubsection{Periostin and Respiratory Disease}

Periostin is likely to play less of a role in lung development as it does in airway repair. Periostin null mice show grossly intact airways, with no significant abnormality. ${ }^{117}$ In neonatal lung remodelling, periostin is upregulated after prolonged hyperoxia leading to dysregulation of alveolar remodelling and septal formation inhibition. ${ }^{144}$ In the lung, periostin expression falls after injury and only increases in response to TGF- $\beta$ activation, ${ }^{16}$ which may persist beyond the initial insult. 
The POSTN gene was identified as a key gene in those with steroid sensitive Type 2 asthma ${ }^{50,51}$ and since then, there has been growing evidence linking periostin to Type 2 airway inflammation. ${ }^{50,51,53,98}$ Periostin is upregulated by Type 2 cytokines IL-4 and IL-13 via activation of Signal Transducer and Activator of Transcription-6 (STAT-6)..$^{50,51,145}$ Its levels are elevated in the bronchial epithelial cells of a subset of asthmatics and is secreted basloaterally. ${ }^{50,145}$ Here, it activates TFG- $\beta$-mediated Type 1 collagen production in fibroblasts ${ }^{145}$ contributing to sub-epithelial fibrosis and airway remodelling, key pathological features of asthma. It is therefore unsurprising that periostin is also upregulated in fibrotic lung diseases such as usual interstitial pneumonitis (UIP) ${ }^{146}$ and high levels are inversely correlated with lung function. ${ }^{147}$

In asthma, periostin is associated with airway eosinophilia ${ }^{98}$ which may be because it acts as a guide to eosinophils, attracting them to areas of high periostin concentration in the asthmatic airway. ${ }^{148}$ Functionally, high levels of periostin have been associated with greater declines in lung function ${ }^{97,149}$ and higher rates of exacerbations, ${ }^{54,69,150}$ both of which are important outcomes in asthma due to associated morbidity and mortality. Clinical studies investigating the efficacy of monoclonal antibody therapy directed against specific components of the Type 2 inflammatory pathway have shown better response to treatment in those with higher periostin levels..$^{52-54,69}$

As a result of all of these factors, periostin may be able to be used as a surrogate biomarker to identify those with Type 2 asthma. This has important clinical implications particularly for those with severe forms of asthma, refractory to current treatment modalities. Firstly, its use may enable clinicians to identify patients who 
have steroid-sensitive asthma. Secondly, for those in whom conventional treatment has failed, it could help identify potential responders to monoclonal antibody therapies, directed against elements of the Type 2 inflammatory pathway. Finally it may enable clinicians to prognosticate on disease progression, which would aid treatment decisions. However, the role of periostin as a biomarker in asthma is far from clear.

In this thesis, I will attempt to address some questions that have yet to be investigated:

- Given the important role periostin has in bone and dental maintenance, are serum levels affected by bone injury or dental surgery? If so, does this have wider implications for its use as a surrogate biomarker for Type 2 asthma?

- How does serum periostin vary over time in asthma? Is it able to distinguish between stable and unstable asthma?

- Does serum periostin vary between ethnicities?

- How does serum periostin compare to other surrogate biomarkers of asthma, specifically peripheral blood eosinophils, FeNO and serum IgE?

- Is serum periostin able to predict who is more likely to suffer from severe asthma exacerbations, as this group of asthmatics are most likely to develop long-term sequelae of chronic airway inflammation and have the largest use of healthcare within the asthmatic population? 


\subsection{Aims}

The main aims of this research were to:

1. Determine the variability of serum periostin after bone injury and dental surgery in non-asthmatic people.

2. Observe the variations in serum periostin in stable asthma and across ethnicities;

3. Observe variations in serum periostin after severe exacerbations of asthma and initiation of treatment; and over years in a general population of adults with asthma

\subsection{Thesis Outline}

Firstly, I aimed to determine the change in serum periostin in non-asthmatic people who sustained bone injury or who had dental surgery. This was to establish the extent of the effect that these events would have on serum levels, as this would impact the utility of serum periostin as a biomarker in asthma within a clinical setting. I therefore conducted two studies, described in Chapter 2:

- Study 1: a cohort study which recruited 102 participants who sustained a bone fracture or who were scheduled to have hip or knee replacement surgery. Participants were divided into three groups depending on their injury: 'short bone fracture', 'Iong bone fracture' or 'joint replacement'. All participants were 
free of chronic respiratory health conditions. They were followed up for six months and had blood drawn for measurement of serum periostin seven times within that period.

- Study 2: this study recruited 68 participants who were scheduled to have a tooth extraction. Participants were divided into two groups depending on the type of extraction they received: 'simple extraction' and 'surgical extraction'. Again, all participants were not diagnosed with any chronic respiratory condition. Patients were followed up for three months and had blood drawn for measurement of serum periostin seven times within that period.

To investigate the variability of serum periostin in stable asthma and across ethnicities, I undertook a literature review of the evidence to date. I then conducted two studies, detailed in Chapter 3:

- Study 3: a longitudinal cohort study of 60 participants with a doctor's diagnosis of asthma on ICS therapy and at least one other controller. Participants were observed for eight weeks, having blood taken 11 times at pre-defined intervals for serum periostin.

- Study 4: a cohort of 188 Chinese participants were recruited. 120 of them had no history of respiratory disease. 68 participants had a doctor's diagnosis of asthma and were on current treatment. Participants attended for a single visit to have serum periostin measured along with peripheral blood eosinophils, 
FeNO and serum IgE. These results were compared to previous Caucasian cohorts.

Finally, in order to examine the utility of surrogate biomarkers in asthma, I undertook a literature review examining the evidence to date of their use in clinical practice, and how they changed in response to a severe exacerbation of asthma, and its treatment. I also reviewed current evidence on the use of Type 2 biomarkers in predicting severe exacerbations of asthma. I then conducted two studies detailed in Chapter 4:

- Study 5: a cohort of 34 participants were enrolled within 24 hours of starting systemic corticosteroids for a severe exacerbation of their asthma and were observed for 12 weeks. Blood was drawn six times over the study period for measurement of peripheral blood eosinophils, FeNO, serum periostin and serum IgE.

- Study 6: a longitudinal study comprising of two cohorts of asthmatics, who were invited to re-attend for a single visit. Repeat measurements of peripheral blood eosinophils, FeNO, serum periostin and IgE were taken as well as lung function. I also took an 'Exacerbation History' whereby I elicited the number of times the participants had had to receive systemic corticosteroids for control of their asthma in intervening time, which I corroborated with medical records.

The findings are presented here and are submitted to Victoria University of Wellington in fulfilment of the requirements for the degree of Doctor of Philosophy. 


\section{Chapter 2: Variations in serum}

\section{periostin after bone and dental injury}

\subsection{What we know so far regarding periostin in bone growth and healing}

Bone in humans is formed through two types of ossification: intramembranous and endochondral. Intramembranous ossification results in the formation of flat bones in the skull and the addition of bone along the periosteal surface in long bones. Endochondral ossification is responsible for the formation of the rest of the bones in the body. ${ }^{151,152}$ The periosteum, the layer of connective tissue surrounding the bone, is vital to bone growth and health and mediates the necessary cell signalling required for bone growth and remodelling. The periosteum is composed of two layers: an outer, fibrous layer composed of fibroblasts and mesenchymal cells and an inner, osteogenic layer, which includes mesenchymal stem cells, osteoblasts and endothelial pericytes. ${ }^{153}$ Mesenchymal stem cells are able to differentiate into osteoblasts during bone formation or healing ${ }^{154,155}$ and express high levels of periostin during fracture healing. ${ }^{110}$

Bone growth or remodeling is largely driven by the Wnt/LRP5/ $\beta$-catenin signaling pathway within osteoblasts. ${ }^{156}$ Although this pathway can be inhibited by sclerostin 
expression, ${ }^{157}$ its function can be preserved by the expression of periostin, which inhibits sclerostin release from osteocytes. ${ }^{110}$ This signaling pathway stimulates the release of bone growth factors, such as TGF- $\beta$, fibroblast growth factor and BMPs, which are upregulated during times of growth or repair. ${ }^{105,158}$

Murine studies in periostin-deficient mice suggest that periostin does not seem to be integral to embryonic development of bone with no differences between periostin-null embryos and their wildtype equivalents. However, up to $14 \%$ of the periostin-null newborn pups die shortly after birth and all periostin-null mice demonstrate severe growth retardation throughout life, suggesting periostin is required for bone health. ${ }^{117}$ In normal mice, immunochemistry studies using anti-periostin anti-serum to evaluate periostin expression in bone tissue, periostin is strongly expressed in the periosteum but not in bone matrix or endosteum. Furthermore the staining pattern suggests that periostin is concentrated in the extracellular matrix, rather than being anchored to a cell's surface. ${ }^{104}$ This supports the theory that the periosteum contributes significantly to the growth and development of bone and, as periostin is expressed so strongly within the periosteum, it too has an important role in osteogenesis.

Periostin also has a role in bone strength and fracture healing. Mechanical loading or exercise is one way of increasing bone strength and can reduce one's fracture risk by stimulating bone formation along the periosteal surfaces. ${ }^{156,159,160}$ After fractures, periostin expression is rapidly upregulated within the periosteum, which suggests that it acts as a conduit to concentrate biomechanical signals to where they are most needed. ${ }^{110}$ This theory is supported by murine models, which demonstrate that 
periostin-null mice have lower bone mineral density and bone strength, particularly in load-bearing long bones, such as the femur or tibia, ${ }^{110}$ which are prone to fracture. This is likely due to disrupted microarchitecture resulting in shorter long bones and abnormal formation of the cartilaginous growth plate. ${ }^{117}$ The weakness is not improved after exercise in periostin-null mice, when compared with their wild-type counterparts who demonstrate improvements in bone mineral density and strength. ${ }^{110}$

Following a fracture periostin mRNA is upregulated significantly within pre-osteoblastic cells and mesenchymal stem cells close to the fracture site, in murine models. This over-expression of periostin persists for up to seven days post-fracture, with high levels seen within both soft and hard calluses forming over the periosteum. Levels of periostin start to fall after day 14 in mice, when the hard callus has thickened and endochondral ossification starts. ${ }^{119}$ This upregulation of periostin at the fracture site further supports the role of serum periostin in bone healing and remodeling.

\subsection{What we know so far about periostin in dental health}

The PDL sits in between the tooth and the alveolar bone and helps anchor the teeth to the bone during mastication. It consists of two lines of periodontal fibroblasts: connective tissue fibroblasts and osteoblast-like fibroblasts, ${ }^{161}$ which are versatile during injury and can differentiate into many cells. ${ }^{162}$ Periostin was originally found to be highly expressed within the PDL, particularly within fibroblasts, suggesting it had a role in the function of the PDL. ${ }^{104}$ 
Once again, much of what is known about periostin's function in dental health comes from murine models. In the developing foetus, periostin-deficiency does not seem to result in any gross abnormalities of dentition. In the newborn period, periostin-null mouse pups have normal developing teeth and periodontia with periostin expression concentrated around developing teeth at the epithelial-mesenchymal junctions. ${ }^{163}$ However, by four weeks abnormalities start to show with widening of the PDL and resorption of bone occurring at tooth roots. At three months, periostin-null mice develop a periodontitis-like disorder characterised by alveolar bone destruction and external root absorption with high osteoclastic activity. ${ }^{117}$ Upon eruption of the teeth, incisors in particular have severe enamel defects with an irregular pattern which are prone to breaking, resulting in poor feeding by the mice and subsequent malnourishment and poor weight gain. Even when the forces of mastication were somewhat ameliorated by the introduction of soft or pureed food, mice still demonstrate features of this periodontitis-like disorder, suggesting that periostin is required to mediate the stressors placed on teeth and jaw attachments. ${ }^{117}$

Experimental tooth movement in rats causes increased expression of periostin in the PDL at the pressure site, ${ }^{164}$ along with an increased concentration of dead cells at the point of stress in periostin-null mice. ${ }^{113}$ Taken together, this supports previous evidence that periostin plays an important role in integrity of the PDL in response to stress, but also that it also prevents apoptosis of cells when under pressure. 
The functions of periostin in bone and dental health and disease are becoming clearer. However, there remains a paucity of data in human subjects. In addition, much of what has been studied in vivo has referred to localised periostin, not serum concentrations. Given the potential use of periostin as a non-invasive biomarker of Type 2 asthma, it would be important to understand what other factors other than those related to Type 2 inflammation and its treatment, can affect serum periostin levels. This is an important consideration as periostin is a matricellular protein which influences tissue development, remodeling and repair in humans. ${ }^{165}$ 


\subsection{Study One: Change in serum periostin after joint replacements, long bone fractures or short bone fractures}

\subsubsection{Introduction}

Periostin plays an integral role in the growth, remodelling and repair of bone and is significantly upregulated following fracture. However, there are no studies conducted in humans describing the changes in serum periostin following bone fracture. Changes to serum periostin under these conditions would affect how levels are interpreted if serum periostin was to be used as a biomarker clinically in asthma.

\subsubsection{Hypothesis}

It was hypothesised that serum periostin would increase following bone injury, and then fall to stable levels within a six month period. The magnitude and duration of periostin increase would be greater in those with fractures in larger bones.

\subsubsection{Objectives}

To determine:

- The magnitude of change in serum periostin following a large joint replacement, long bone fracture and short bone fracture;

- The difference in magnitude of change between the three groups; 
- The time taken for serum periostin to return to pre-operative levels in the joint replacement group, as this was the only group to have a pre-operative measurement. 


\subsubsection{Methods}

This cohort study recruited 102 patients aged 18 to 75 years from the emergency department, orthopaedic fracture clinic, and pre-surgical assessment clinic at Wellington Regional Hospital, Wellington, New Zealand. Three groups of participants were recruited: those with short bone fractures, long bone fractures, and those scheduled for hip or knee arthroplasty. 'Short bones' included carpal, metacarpal, tarsal, metatarsal, or vertebral fractures. 'Long bones' included tibia, fibula, femur, humerus, radial, ulnar, or rib fractures. Participants were excluded from the study if they had conditions with the theoretical potential to affect serum periostin levels. These included: a doctor's diagnosis of asthma, bronchitis or chronic obstructive pulmonary disease (COPD); wheeze or use of respiratory inhalers in the previous 12 months; hospital admission, significant surgery (including dental surgery), bone fracture, or use of systemic corticosteroids, all within the three months prior to enrolment. Participants were also not recruited if they were pregnant or breastfeeding.

Ethical approval was given by the Central Regional Ethics Committee of New Zealand (13/NTB/186). The trial was prospectively registered with the Australia New Zealand Trials Registry (ACTRN12614000151639) and written informed consent was obtained from all participants prior to testing. The full information sheet and protocol are included in Appendices I and II. 
Participants attended the Medical Research Institute of New Zealand (MRINZ) outpatient facility over a 26-week period. Participants with fractures were enrolled within 48 hours of their injury and then attended for visits at times of one, two, four, eight, 12 and 26 weeks after the fracture. Participants undergoing joint replacement surgery had an additional visit before the surgical procedure to obtain a sample for pre-operative serum periostin, and then were followed-up with the same visit schedule as the fracture groups.

At enrolment, participants completed a General Health Questionnaire (Appendix IX), which was based on questions from the American Thoracic Society (ATS) Division of Lung Diseases-78 Questionnaire (DLD-78) ${ }^{166}$ and measurement of their body mass index (BMI). Serum periostin was measured at every study visit, including the enrolment visit. Serum periostin was measured using the clinical trial version of the Elecsys ${ }^{\circledR}$ Periostin Immunoassay (Roche Diagnositics, Penzbery, Germany) described previously. ${ }^{115}$

Sample size

The clinically important difference in serum periostin is not known. The sample size of 34 participants in each sub-group was based on detecting a paired difference in periostin of 0.5 of a standard deviation ( $80 \%$ power, Type I error rate $5 \%$, two-sided), which can be considered a 'medium' effect size. For comparison between long and short bone fractures groups, a sample size of 68 (34 in each group) was based on $90 \%$ 
power to detect a 0.8 standard deviation difference, which can be considered a 'large' effect size.

\section{Statistical methods}

The distribution of serum periostin was right skewed and therefore the logarithmic data transformation was applied on the values for analysis. The inverse transformation of a difference in logarithms is equivalent to a ratio of geometric means. Periostin values were plotted against time and a locally weighted scatter plot smoother (LOESS) with $90 \%$ confidence limits was used to illustrate the pattern of variation with time. Comparison between patient groups used mixed linear models with the individual participant as a random effect and a spatial exponential covariance matrix, using the time between measurements, to estimate the correlation between repeated measurements. Two sets of comparisons were estimated. Firstly, within group models were used to estimate the change from the reference measurement for each patient group; secondly an interaction between patient group and time was assessed. In the fracture groups, as a pre-fracture serum sample could not be obtained from participants, the initial analysis was performed using the first periostin measurement (taken within 48 hours) as the reference measurement. However when it was shown that serum periostin levels fell within 48 hours of bone injury associated with joint replacement surgery, the 48 hour periostin levels could not be used as the reference measurement in the fracture groups. For this reason, a post hoc analysis was conducted where within group comparisons were estimated using the 26 week values as the reference measurement. 
SAS version 9.4 was used.

\subsubsection{Results}

A total of 102 participants were recruited after 415 people were screened. Common reasons for screen failures included being outside of the designated age range, sustaining a fracture more than 48 hours prior to screening, declining to participate, and having a doctor's diagnosis of asthma or COPD. A single participant was misallocated to the short bone fracture group with an iliac fracture and so was excluded from analysis. The characteristics of study participants are shown in Table 2.1. The participants in the joint replacement group were older and had a higher BMI than those in the fracture groups. 
Table 2.1: Baseline participant characteristics for all participants in Study One

\begin{tabular}{|c|c|c|c|}
\hline & $\begin{array}{l}\text { Joint } \\
\text { Replacement }\end{array}$ & $\begin{array}{l}\text { Long Bone } \\
\text { Fractures }\end{array}$ & Short Bone Fractures \\
\hline \multicolumn{4}{|c|}{ Continuous variables: $\mathrm{N}=34$} \\
\hline Age $^{1}:$ mean (SD) & $60.6(10.1)$ & $47.9(17.4)$ & $39.8(15.7)$ \\
\hline $\mathrm{BMI}^{2}$ : mean (SD) & $31.5(8.3)$ & $26.2(3.7)$ & $27.4(4.9)$ \\
\hline \multicolumn{4}{|c|}{ Categorical variables $\mathrm{N}=34$} \\
\hline Female (\%) & $16(47)$ & $21(62)$ & $12(35)$ \\
\hline Atopy $^{3}(\%)$ & $17(50)$ & $18(53)$ & $16(47)$ \\
\hline Non-smoker (\%) & $31(91)$ & $29(85)$ & $27(79)$ \\
\hline \multicolumn{4}{|l|}{ Ethnicity } \\
\hline European (\%) & $24(71)$ & $27(79)$ & $28(82)$ \\
\hline Maori (\%) & $6(18)$ & $5(15)$ & $3(8)$ \\
\hline Pacific (\%) & $2(6)$ & $0(0)$ & $1(3)$ \\
\hline Asian (\%) & $1(3)$ & $1(3)$ & $1(3)$ \\
\hline Other (\%) & $1(3)$ & $1(3)$ & $1(3)$ \\
\hline
\end{tabular}

Steroid medication ${ }^{4}$

$\begin{array}{llll}\text { Oral steroids (\%) } & 1(3) & 0(0) & 0(0)\end{array}$

Intranasal steroids (\%) 1 (3) $\quad 0$ (0) $4(12)$

$\begin{array}{llll}\text { Topical (\%) } & 0(0) & 0(0) & 1(3)\end{array}$

1 Age: years
2 BMI: $\mathrm{kg} / \mathrm{m} 2$
3 Atopy defined as a history of seasonal rhinoconjunctivitis and/or eczema
4 Participants who took corticosteroid containing medication for any reason during the study period
SD: standard deviation, IQR: interquartile range, BMI: body mass index, ng: nanogram, ml: millilitre. 
Joint replacement group

In this group, there were 18 participants who underwent hip replacement surgery and 16 who had knee replacement surgery, with complete data available on 31 participants. The mean (SD) length of time between the pre-operative reference measurement and the surgery was 46.6 (36.9) days (range 0 to 170 days). The preoperative mean (SD) serum periostin was $54.2(18.0) \mathrm{ng} / \mathrm{ml}$.

Within 48 hours of surgery, serum periostin levels fell to a mean (SD) of 43.5 (12.5) ng/ $\mathrm{ml}$, represented by a ratio of geometric mean periostin of $0.8, \mathrm{P}<0.001$ (Table 2.2). Serum periostin levels returned to baseline one week post-operatively and then progressively increased, with a maximum mean (SD) of $101.3(31.2) \mathrm{ng} / \mathrm{ml}$, (difference $46.9 \mathrm{ng} / \mathrm{ml}$, ratio of geometric mean periostin $1.89, \mathrm{P}<0.001)$ at 8 weeks. The periostin level then decreased, but remained above the pre-operative reference level at week 26, when the mean (SD) periostin level was $68.3(20.7) \mathrm{ng} / \mathrm{ml}$, difference $13.8 \mathrm{ng} / \mathrm{ml}$, ratio of geometric mean periostin 1.27, $\mathrm{P}<0.001$ (Figure 2.1). 
Table 2.2: Serum periostin levels at time points in joint replacement group, and ratio of geometric means compared with pre-operative reference baseline value

\begin{tabular}{|c|c|c|c|c|c|}
\hline Visit & $\mathbf{N}$ & $\begin{array}{l}\text { Periostin } \\
\text { Mean (SD) }\end{array}$ & $\begin{array}{l}\text { Change } \\
\text { from } \\
\text { Baseline } \\
\text { Mean (SD) }\end{array}$ & $\begin{array}{l}\text { Ratio of geometric } \\
\text { means }(95 \% \mathrm{Cl})\end{array}$ & $\mathbf{P}$ \\
\hline $\begin{array}{l}\text { Pre-operative } \\
\text { (reference) }\end{array}$ & 34 & $54.2(18.0)$ & - & - & - \\
\hline $\begin{array}{c}\text { Within } 48 \\
\text { hours }\end{array}$ & 31 & $43.5(12.5)$ & $-11.5(9.7)$ & $0.80(0.75$ to 0.86$)$ & $<0.001$ \\
\hline Week 1 & 33 & $55.5(17.8)$ & $1.4(12.4)$ & $1.03(0.97$ to 1.10$)$ & 0.37 \\
\hline Week 2 & 32 & $79.0(27.0)$ & $24.0(17.3)$ & $1.44(1.35$ to 1.54$)$ & $<0.001$ \\
\hline Week 4 & 32 & $97.3(29.2)$ & $43.6(1.9)$ & $1.83(1.72$ to 1.96$)$ & $<0.001$ \\
\hline Week 8 & 31 & $101.3(31.2)$ & $46.9(22.6)$ & 1.89 (1.77 to 2.02 ) & $<0.001$ \\
\hline Week 12 & 31 & $89.8(26.6)$ & $35.3(16.3)$ & 1.67 (1.57 to 1.79$)$ & $<0.001$ \\
\hline Week 26 & 31 & $68.3(20.7)$ & $13.8(12.8)$ & $1.27(1.19$ to 1.36$)$ & $<0.001$ \\
\hline
\end{tabular}


Figure 2.1: Time course of serum periostin levels over 26 weeks in participants who underwent large joint replacements (including pre-operative reference periostin values). The red solid line denotes the mean and the red dotted lines denote the $90 \%$ confidence intervals.

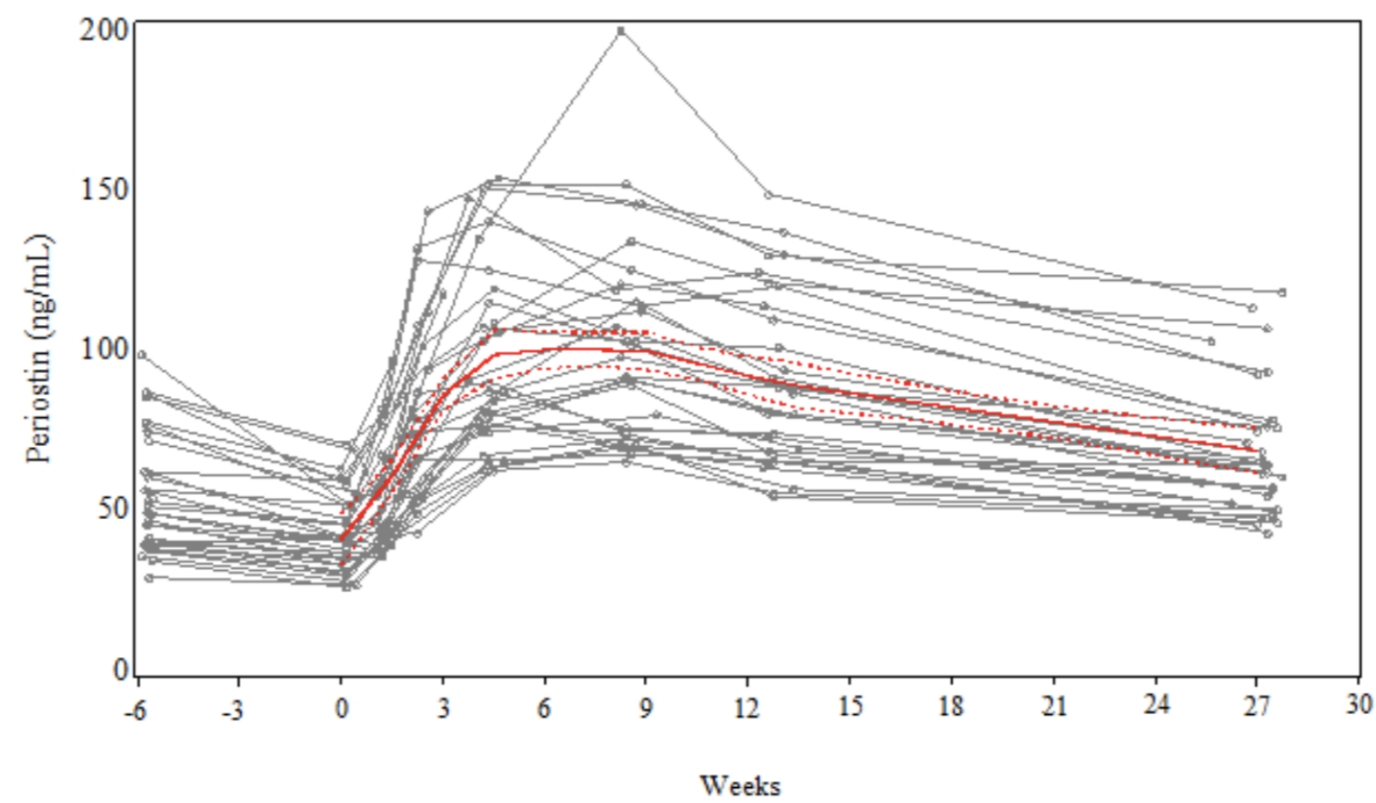


Long bone fracture group

In this group, 22 participants had sustained lower limb long bone fractures and 12 sustained upper limb long bone fractures. In 13 participants there were two or more fractures, and there was complete data in 30 participants. The mean (SD) length of time between fracture and the first periostin sample was 1.1 (0.59) days, (range 0 to 2 days). Given the fall in serum periostin level within 48 hours after joint arthroplasty, a post hoc analysis was conducted using the 26 week value as the reference measurement. The mean (SD) periostin at 26 weeks was $56(16.3) \mathrm{ng} / \mathrm{ml}$.

Compared to the 26 week reference value, the mean (SD) periostin level at 48 hours after fracture was lower at $42.7(10.6) \mathrm{ng} / \mathrm{ml}$, (difference $-13.4 \mathrm{ng} / \mathrm{ml}$, ratio of geometric mean periostin 0.76, $\mathrm{P}<0.001$ ) (Table 2.3). Periostin levels at week two were similar to the 26 week reference values, and then increased progressively to a maximum of $66.6(25.1) \mathrm{ng} / \mathrm{ml}$ at week 8 (difference $11 \mathrm{ng} / \mathrm{ml}$, ratio of geometric mean periostin 1.15, $\mathrm{P}<0.001$ ) (Figure 2.2). 
Table 2.3: Serum periostin levels at time points in long bone fracture group, and ratio of geometric means compared with week 26 reference level

\begin{tabular}{|c|c|c|c|c|c|}
\hline Visit & $\mathbf{N}$ & $\begin{array}{l}\text { Periostin } \\
\text { Mean (SD)* }\end{array}$ & $\begin{array}{l}\text { Change from } \\
\text { Baseline } \\
\text { Mean (SD)* }\end{array}$ & $\begin{array}{c}\text { Ratio of } \\
\text { geometric } \\
\text { means }(95 \% \mathrm{CI})\end{array}$ & $\mathbf{P}$ \\
\hline $\begin{array}{l}\text { Within } 48 \\
\text { hours }\end{array}$ & 34 & $42.7(10.6)$ & $-13.4(12.4)$ & $\begin{array}{c}0.76(0.71 \text { to } \\
0.83)\end{array}$ & $<0.001$ \\
\hline Week 1 & 34 & 45.7 (11.9) & $-11.2(10.9)$ & $\begin{array}{c}0.81(0.76 \text { to } \\
0.88)\end{array}$ & $<0.001$ \\
\hline Week 2 & 34 & $52.5(13.0)$ & $-3.7(8.2)$ & $\begin{array}{c}0.94(0.87 \text { to } \\
1.02)\end{array}$ & 0.12 \\
\hline Week 4 & 33 & $62.0(18.2)$ & $-5.8(10.9)$ & $\begin{array}{c}1.10(1.02 \text { to } \\
1.18)\end{array}$ & 0.015 \\
\hline Week 8 & 34 & $66.6(25.1)$ & $-11.0(16.1)$ & $\begin{array}{c}1.15(1.06 \text { to } \\
1.23)\end{array}$ & $<0.001$ \\
\hline Week 12 & 32 & $63.9(21.9)$ & $-7.7(10.9)$ & $\begin{array}{c}1.11(1.04 \text { to } \\
1.20)\end{array}$ & 0.004 \\
\hline $\begin{array}{l}\text { Week } 26 \\
\text { (reference) }\end{array}$ & 30 & $56.0(16.3)$ & - & - & - \\
\hline
\end{tabular}


Figure 2.2: Time course of serum periostin levels over 26 weeks in participants who sustained a long bone fracture. The red solid line denotes the mean and the red dotted lines denote the $90 \%$ confidence intervals.

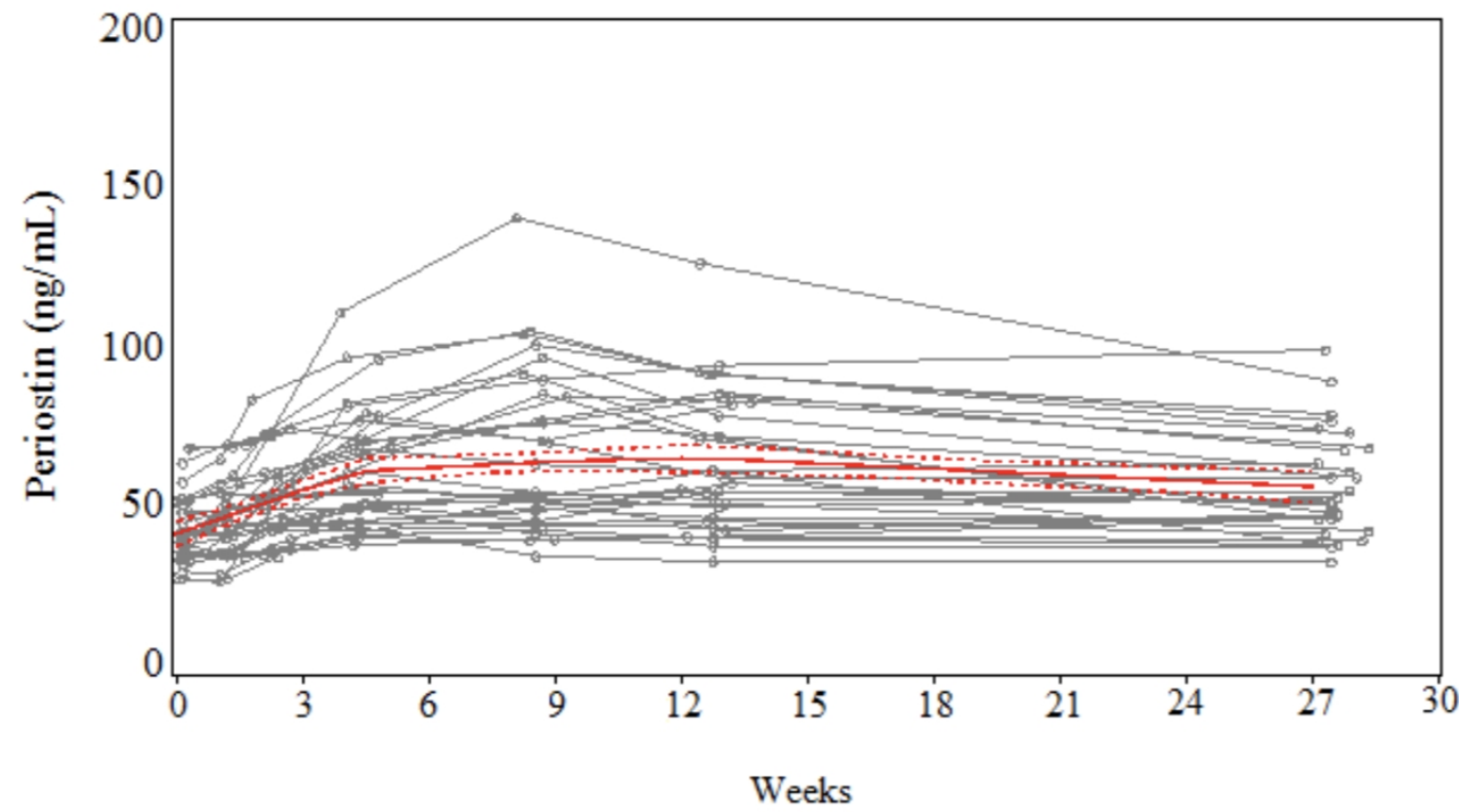


Short bone fracture group

Of the 34 participants recruited, 24 had complete data. Within this group: nine had sustained a metacarpal or carpal fracture, five had a metatarsal or tarsal fracture, 16 people had fractured phalanges (11 in the hand, five in the foot), two people had calcaneus fractures and one person fractured their talus. Three participants had sustained two or more fractures. The mean (SD) length of time between fracture and the first periostin sample was 1.29 (0.68) days, (range 0 to 2 days). The mean (SD) periostin at 26 weeks was $53(14.8) \mathrm{ng} / \mathrm{ml}$.

Compared with the 26 week reference value, the periostin level at 48 hours was lower, with a mean (SD) of $46.1(10) \mathrm{ng} / \mathrm{ml}$ (difference $-5.7 \mathrm{ng} / \mathrm{ml}$, ratio of geometric mean periostin 0.9, $\mathrm{P}<0.001$ ) (Table 2.4). Periostin levels at week two were similar to the 26 week measurements (mean $49.9 \mathrm{ng} / \mathrm{ml}$, difference $-2.4 \mathrm{ng} / \mathrm{ml}$, ratio of geometric mean periostin $0.97, \mathrm{P}=0.37$ ) and did not change significantly after this time point (Figure 2.3). 
Table 2.4: Serum periostin levels at time points in short bone fracture group, and ratio of geometric means compared with week 26 reference level

\begin{tabular}{|c|c|c|c|c|c|}
\hline Visit & $\mathbf{N}$ & $\begin{array}{l}\text { Periostin } \\
\text { Mean } \\
\text { (SD) }{ }^{*}\end{array}$ & $\begin{array}{c}\text { Change from } \\
\text { Baseline } \\
\text { Mean (SD) }\end{array}$ & $\begin{array}{l}\text { Ratio of geometric } \\
\text { means }(95 \% \mathrm{Cl})\end{array}$ & $\mathbf{P}$ \\
\hline $\begin{array}{l}\text { Within } 48 \\
\text { hours }\end{array}$ & 34 & $46.1(10.0)$ & $-5.7(14.8)$ & $0.90(0.85$ to 0.95$)$ & $<0.001$ \\
\hline Week 1 & 32 & $47.8(9.6)$ & $-3.5(11.2)$ & 0.93 (0.88 to 0.99$)$ & 0.021 \\
\hline Week 2 & 29 & $49.9(12.1)$ & $-2.4(7.8)$ & 0.97 (0.93 to 1.03$)$ & 0.37 \\
\hline Week 4 & 28 & $51.5(14.2)$ & $0.5(7.3)$ & $1.00(0.95$ to 1.06$)$ & 0.87 \\
\hline Week 8 & 27 & $50.6(15.7)$ & $-1.0(6.9)$ & $0.97(0.92$ to 1.02$)$ & 0.24 \\
\hline Week 12 & 27 & $51.6(12.1)$ & $-0.3(6.9)$ & $1.00(0.94$ to 1.05$)$ & 0.99 \\
\hline $\begin{array}{l}\text { Week } 26 \\
\text { (reference) }\end{array}$ & 24 & $53.0(14.8)$ & - & - & - \\
\hline
\end{tabular}


Figure 2.3: Time course of serum periostin levels over 26 weeks in participants who sustained a small bone fracture. The red solid line denotes the mean and the red dotted lines denote the $90 \%$ confidence intervals.

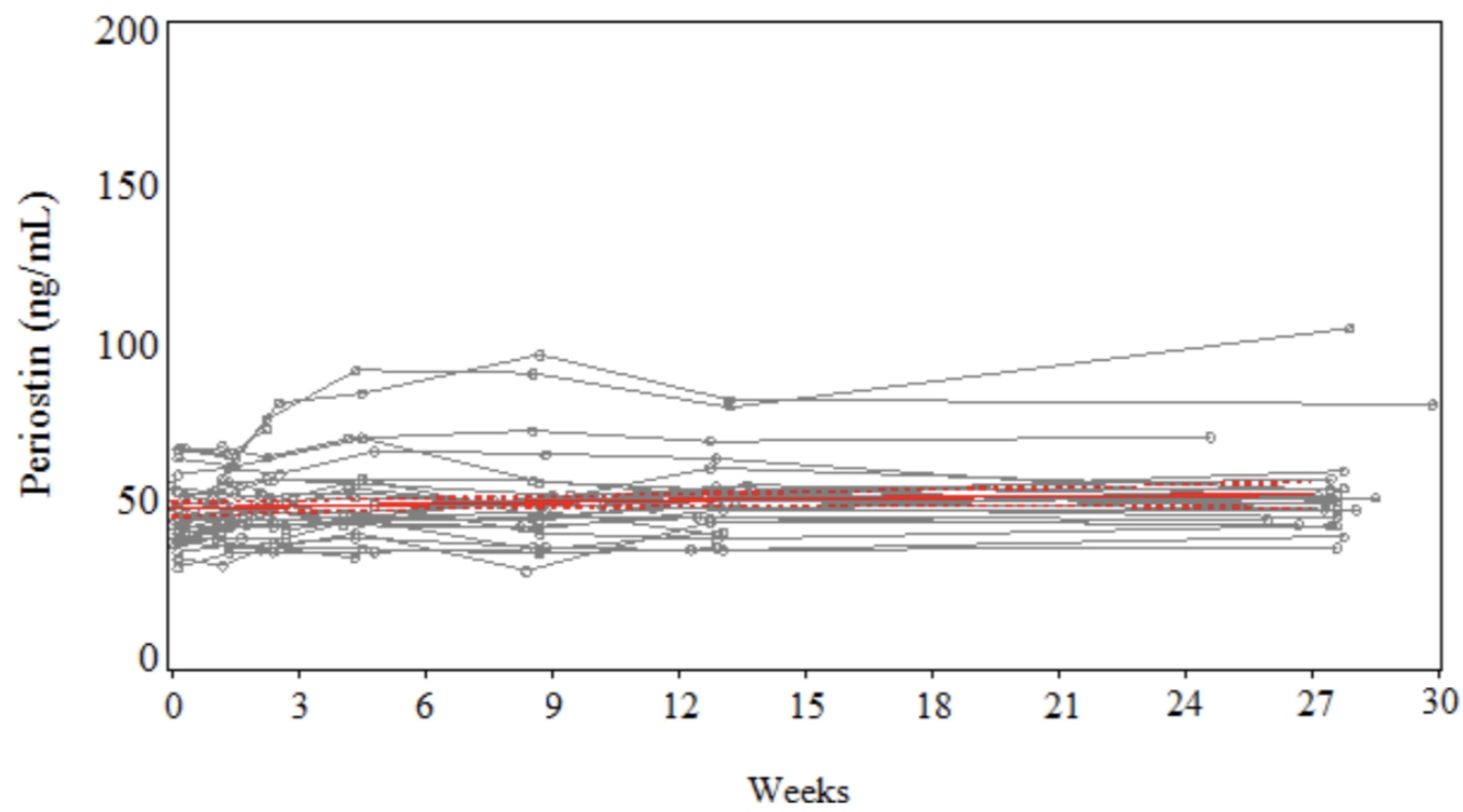


Comparison between the groups

The periostin levels were similar between the joint replacement and long fracture groups at 48 hours, but the joint replacement group had higher periostin levels at all subsequent time points to week 26 (Table 2.5).

The periostin levels were similar between the long bone fracture and short bone fracture groups at 48 hours to week 2. The long bone fracture group had higher periostin levels at weeks 4,8 and 16 , but there was no difference between the two fracture groups at week 26 (Table 2.5). Raw serum periostin values, for all three groups and all visits, are shown in Tables 2.6, 2.7 and 2.8. 
Table 2.5: Change in serum periostin over time: differences between the three groups

Joint replacement minus Long bone fracture

\begin{tabular}{llll}
\hline Visit & $\begin{array}{l}\text { Difference in Logarithm } \\
\text { Periostin }(95 \% \mathrm{Cl})\end{array}$ & $\begin{array}{c}\text { Ratio of geometric mean } \\
\text { periostin }(95 \% \mathrm{Cl})\end{array}$ & P \\
\hline Within 48 hours & $-0.01(-0.14$ to 0.12$)$ & $0.99(0.87$ to 1.13$)$ & 0.90 \\
Week 1 & $0.18(0.05$ to 0.31$)$ & $1.20(1.05$ to 1.37$)$ & 0.007 \\
Week 2 & $0.37(0.24$ to 0.51$)$ & $1.45(1.27$ to 1.66$)$ & $<0.001$ \\
Week 4 & $0.47(0.34$ to 0.60$)$ & $1.59(1.40$ to 1.82$)$ & $<0.001$ \\
Week 8 & $0.45(0.32$ to 0.58$)$ & $1.57(1.37$ to 1.79$)$ & $<0.001$ \\
Week 12 & $0.36(0.23$ to 0.49$)$ & $1.43(1.25$ to 1.63$)$ & $<0.001$ \\
Week 26 & $0.19(0.06$ to 0.33$)$ & $1.22(1.06$ to 1.39$)$ & 0.004 \\
\hline
\end{tabular}

Long bone fracture minus Short Bone fracture

\begin{tabular}{lccc}
\hline Visit & $\begin{array}{l}\text { Difference in Logarithm } \\
\text { Periostin }(95 \% \mathrm{Cl})\end{array}$ & $\begin{array}{c}\text { Ratio of geometric mean } \\
\text { periostin }(95 \% \mathrm{Cl})\end{array}$ & \\
\hline Within 48 hours & $-0.09(-0.22$ to 0.04$)$ & $0.92(0.80$ to 1.04$)$ & 0.18 \\
Week 1 & $-0.05(-0.18$ to 0.08$)$ & $0.95(0.84$ to 1.08$)$ & 0.45 \\
Week 2 & $0.06(-0.07$ to 0.19$)$ & $1.06(0.93$ to 1.21$)$ & 0.35 \\
Week 4 & $0.19(0.06$ to 0.32$)$ & $1.21(1.06$ to 1.38$)$ & 0.005 \\
Week 8 & $0.28(0.14$ to 0.41$)$ & $1.32(1.15$ to 1.50$)$ & $<0.001$ \\
Week 12 & $0.21(0.07$ to 0.34$)$ & $1.23(1.08$ to 1.40$)$ & 0.002 \\
Week 26 & $0.11(-0.03$ to 0.24$)$ & $1.11(0.97$ to 1.27$)$ & 0.11 \\
\hline
\end{tabular}


Table 2.6. Raw serum periostin (ng/ml) values for Joint Replacement Group

\section{Visit number}

\begin{tabular}{|c|c|c|c|c|c|c|c|c|}
\hline & Pre- & & & & & & & \\
\hline ID & $\begin{array}{l}\text { operative } \\
(\mathrm{N}=34)\end{array}$ & $1(\mathrm{~N}=31)$ & $2(\mathrm{~N}=33)$ & $3(\mathrm{~N}=32)$ & $4(\mathrm{~N}=32)$ & $5(\mathrm{~N}=31)$ & $6(N=31)$ & $7(\mathrm{~N}=31)$ \\
\hline 1 & 77.24 & 62.75 & 96.42 & 143.2 & 153.5 & 145.5 & 136.6 & 92.17 \\
\hline 2 & 85.09 & 68.58 & 54.37 & 81.39 & 134.5 & 199.8 & 148.4 & 113 \\
\hline 3 & 49.13 & 39.58 & 36.37 & 56.98 & 86.27 & 114.9 & 91.22 & 61.52 \\
\hline 4 & 61.97 & 59.16 & 81.57 & 131.6 & 139.9 & 124.9 & 109.3 & 77.95 \\
\hline 5 & 49.14 & 37.53 & 44.52 & 66.45 & 74.1 & 79.48 & 69.05 & 56.97 \\
\hline 6 & 39.26 & & 36.21 & 65.69 & 65.42 & 67.17 & 64.79 & 45.19 \\
\hline 7 & 45.19 & & 39.12 & 54.11 & 80.37 & 90.39 & 79.68 & 64.32 \\
\hline 8 & 48.46 & 40.39 & 50.41 & 91.55 & 106.9 & 102.6 & 79.9 & 61.54 \\
\hline 9 & 35.41 & 27.47 & 41.65 & 42.67 & 62.23 & 64.81 & 55.16 & 47.76 \\
\hline 10 & 52.71 & 45.66 & 64.99 & 78.15 & 86.98 & 75.61 & 72.55 & 49.77 \\
\hline 11 & 46.55 & 32.78 & 38.2 & 65.39 & 74.71 & 90.9 & 80.63 & 65.54 \\
\hline 12 & 74.48 & 59.88 & 83.1 & 111.9 & 147.2 & 118.5 & 123.9 & 93.05 \\
\hline 13 & 38.55 & 42.5 & 42.75 & 55.07 & 80.94 & 69.45 & 68.41 & 56.28 \\
\hline 14 & 55.69 & 40.7 & 45.36 & 73.81 & 76.4 & 73.64 & 64.97 & 64.13 \\
\hline 15 & 39.57 & 32.78 & 38.42 & 51.79 & 66.87 & 72.51 & 64.78 & 51.95 \\
\hline 16 & 61.4 & 41.1 & 47.33 & 71.31 & 108.1 & 133.8 & 120.8 & 106.6 \\
\hline 17 & 37.44 & 30.66 & 44.91 & 52.65 & 77.63 & 69.28 & 63.09 & 46.89 \\
\hline 18 & 45.32 & 36.27 & 65.79 & 100.9 & 118.9 & 102.6 & 100.5 & 63.65 \\
\hline
\end{tabular}




\begin{tabular}{|c|c|c|c|c|c|c|c|c|}
\hline 19 & 71.47 & 55.06 & 86.19 & 117.5 & & & & \\
\hline 20 & 39.26 & 40.75 & 53.91 & 55.21 & 90.74 & 107.3 & 86.27 & 54.3 \\
\hline 21 & 34.01 & 25.78 & 39.02 & 52.55 & 78.79 & 88.98 & 67.36 & 42.72 \\
\hline 22 & 37.15 & 29.3 & 45.83 & 73.71 & 114.5 & 101.4 & 89.55 & 60.25 \\
\hline 23 & 51.08 & 44.94 & 71.75 & 93.62 & 105.7 & 112.1 & 93.43 & 71.09 \\
\hline 24 & 60.01 & 46.93 & 67.05 & 79.14 & 103 & 120.5 & 113.6 & 74.63 \\
\hline 25 & 28.84 & 26.41 & 42.16 & & 65.06 & & & \\
\hline 26 & 56.27 & 51.26 & 57.18 & 72.6 & 83.97 & 91.54 & 88.22 & 75.96 \\
\hline 27 & 85.66 & 57.95 & 76.45 & 107.5 & 151.7 & 151.4 & 129.4 & 117.8 \\
\hline 28 & 86.83 & 70.28 & 78.49 & 104.8 & 150.5 & 145.1 & 129.7 & 102.7 \\
\hline 29 & 53.57 & & & & & & & \\
\hline 30 & 37.58 & 34.83 & 35.58 & 48.92 & 63.57 & 70.48 & 56.43 & 49.99 \\
\hline 31 & 41.05 & 29.66 & 40.75 & 53.34 & 62.43 & 70 & 54.23 & 45.74 \\
\hline 32 & 41 & 34.8 & 45.25 & 60.28 & 87.17 & 97.71 & 87.5 & 68.15 \\
\hline 33 & 76.91 & 51.32 & 57.88 & 86.39 & 89.64 & 74.55 & 73.74 & 58.75 \\
\hline 34 & 98.3 & 50.78 & 84.05 & 127.9 & 124.8 & 113.6 & 119.8 & 75.61 \\
\hline
\end{tabular}


Table 2.7. Raw serum periostin (ng/ml) values for Long Bone Fracture Group

\begin{tabular}{|c|c|c|c|c|c|c|c|}
\hline \multicolumn{8}{|c|}{ Visit number } \\
\hline ID & $1(\mathrm{~N}=34)$ & $2(\mathrm{~N}=34)$ & $3(\mathrm{~N}=34)$ & $4(\mathrm{~N}=33)$ & $5(\mathrm{~N}=34)$ & $6(\mathrm{~N}=32)$ & $7(\mathrm{~N}=30)$ \\
\hline 1 & 44.78 & 51.26 & 63.05 & 68.79 & 84.41 & 82.62 & 68.28 \\
\hline 2 & 50.47 & 57.12 & 60.8 & 67.92 & 63.33 & 61.25 & 63.69 \\
\hline 3 & 51.44 & 54.24 & 53.18 & 54.66 & 53.35 & 54.23 & 54.78 \\
\hline 4 & 32.94 & 43.08 & 49.47 & 57.27 & 51.39 & 55.32 & \\
\hline 5 & 33.68 & 53.57 & 52.71 & 53.91 & & & \\
\hline 6 & 37.99 & 35.1 & 36.62 & 40.15 & 39.59 & 40.34 & 46.13 \\
\hline 7 & 68.4 & 69.26 & 74.49 & 70.6 & 76.12 & 84.37 & 73.15 \\
\hline 8 & 52 & 56.91 & 59.2 & 66.06 & 76.74 & 85.61 & 67.29 \\
\hline 9 & 34.56 & 36.52 & 45.81 & 51.22 & & 50.71 & 42.28 \\
\hline 10 & 39.93 & 45.29 & 44.43 & 44.73 & 44.96 & 45.41 & 41.3 \\
\hline 11 & 38.73 & 43.72 & 53.97 & 69.46 & 76.29 & 71.99 & 59.18 \\
\hline 12 & 31.74 & 34.94 & 44.57 & 43.22 & 34.28 & 32.74 & 32.4 \\
\hline 13 & 29.43 & 28.43 & 36.54 & 41.12 & 42.53 & 39.76 & 37.9 \\
\hline 14 & 34.56 & 35.12 & 34.81 & 37.77 & 39.44 & 40.43 & 39.24 \\
\hline 15 & 33.37 & 34.8 & 42.81 & 49.26 & 41.61 & 37.45 & 36.91 \\
\hline 16 & 42.37 & 41.27 & 57 & 78.04 & 100.7 & 91.69 & 76.68 \\
\hline 17 & 42.38 & 49.14 & 60.84 & 70.63 & 96.54 & 78.52 & 60.92 \\
\hline 18 & 27.33 & 27.12 & 33.86 & 44.71 & 48.46 & 57.08 & 50.46 \\
\hline 19 & 41.69 & 46.77 & 49.18 & 49.45 & 45.42 & 54.47 & 51.24 \\
\hline
\end{tabular}




\begin{tabular}{|c|c|c|c|c|c|c|c|}
\hline 20 & 48.5 & 48.76 & 52.35 & 55.43 & 52.78 & 49.59 & 52.62 \\
\hline 21 & 37.39 & 40.49 & 42.39 & 42.15 & 44.41 & 43.3 & 47.36 \\
\hline 22 & 68.09 & 68.69 & 72.05 & 76.73 & 70.3 & 81.79 & \\
\hline 23 & 35.9 & 34.57 & 35.81 & & 52.57 & 61.18 & 38.63 \\
\hline 24 & 47.57 & 46.76 & 52.95 & 60.48 & 54.41 & 52.19 & 51.6 \\
\hline 25 & 63.56 & 69.31 & 70.54 & 96 & 104.9 & 91.43 & 78.77 \\
\hline 26 & 39.68 & 50.34 & 72.77 & 110.8 & 140.5 & 126.2 & 89.06 \\
\hline 27 & 41 & 40.77 & 57.79 & 79.09 & 70.1 & 58.43 & 58.93 \\
\hline 28 & 47.92 & 39.8 & 50.55 & 60.54 & 85.2 & 70.92 & 47.81 \\
\hline 29 & 27.75 & 26.34 & 43.02 & 82.45 & 91.59 & 71.67 & 45.8 \\
\hline 30 & 40.81 & 41.56 & 43.6 & 49.9 & 52.36 & 42.56 & 46.95 \\
\hline 31 & 34.58 & 33.79 & 39.72 & 45.15 & 49.4 & 46.36 & 46.34 \\
\hline 32 & 41.47 & 44.59 & 43.56 & 49.57 & 54.26 & & \\
\hline 33 & 52.41 & 59.8 & 72.3 & 81.33 & 89.65 & 94.17 & 98.97 \\
\hline 34 & 57.71 & 64.86 & 83.4 & 96.72 & 103.8 & 92 & 74.5 \\
\hline
\end{tabular}


Table 2.8. Raw periostin (ng/ml) values for Short Bone Fracture Group

\begin{tabular}{|c|c|c|c|c|c|c|c|}
\hline \multicolumn{8}{|c|}{ Visit number } \\
\hline ID & $1(\mathrm{~N}=33)$ & $2(\mathrm{~N}=31)$ & $3(\mathrm{~N}=28)$ & $4(N=27)$ & $5(N=26)$ & $6(\mathrm{~N}=26)$ & $7(\mathrm{~N}=25)$ \\
\hline 1 & 58.94 & 61.29 & 58.91 & 66.46 & 65.58 & 64.18 & 50.41 \\
\hline 2 & 34.34 & 36.36 & 37.13 & 38.74 & 36.1 & 35.18 & 35.79 \\
\hline 3 & 51.49 & 56.86 & 57.06 & 57.26 & 56.94 & 52.26 & 59.96 \\
\hline 4 & 40.4 & & & & & & \\
\hline 5 & 46 & 46.81 & 50.31 & 57.7 & 45.8 & 48.75 & 45.48 \\
\hline 6 & 64.32 & 61.75 & & 70.61 & 56.46 & 53.77 & 54.68 \\
\hline 7 & 37.98 & 42.97 & 42.43 & 45.26 & 52.35 & 53.72 & 57.77 \\
\hline 8 & 47.56 & & & & & & \\
\hline 9 & 43 & 41.55 & 46.6 & 46.72 & 49.81 & 52.31 & \\
\hline 10 & 55.42 & 52.57 & 52.4 & 53.39 & 50.48 & 55.73 & 51.99 \\
\hline 11 & 42.5 & 42.09 & 42.89 & 45.24 & 47.27 & 39.94 & 43.59 \\
\hline 12 & 37.4 & 45.3 & 49.99 & 45.89 & 50.8 & 53.23 & 49.87 \\
\hline 13 & 29.43 & 33.92 & 34.58 & 34.43 & 35.69 & 35.04 & 39.29 \\
\hline 14 & 44.52 & 44.64 & 47.61 & 54.64 & 48.93 & 50.53 & 47.92 \\
\hline 15 & 32.46 & 30.11 & 36.11 & 32.62 & & & \\
\hline 16 & 38.93 & 38.43 & 35.13 & 40.17 & 28.58 & 43.99 & 43.17 \\
\hline 17 & 66.79 & 67.77 & 64.35 & 70.55 & 72.9 & 69.74 & 70.98 \\
\hline 18 & 50.46 & 48.94 & 42.85 & 42.46 & 45.5 & 44.75 & \\
\hline 19 & 55.51 & 50.79 & 51.52 & 49.07 & 50.67 & 51.48 & \\
\hline
\end{tabular}




\begin{tabular}{|c|c|c|c|c|c|c|c|}
\hline 20 & 67.35 & 65.26 & 73.51 & & & & \\
\hline 21 & 48.4 & 44.71 & & & & & \\
\hline 22 & 37.5 & 43.84 & 53.31 & & & & \\
\hline 23 & 42.09 & 46.78 & 41.39 & 47.23 & 40.27 & 38.33 & 42.77 \\
\hline 24 & 38.29 & 40.84 & 48 & 44.55 & 45.51 & 45.23 & 42.62 \\
\hline 25 & 67.2 & 64.15 & 81.87 & 84.82 & 97.02 & 82.74 & 81.15 \\
\hline 26 & 37.68 & 35.91 & 35.98 & 35.51 & 33.95 & 35.61 & \\
\hline 27 & 52.1 & 50.42 & 57.72 & 56.09 & 52.43 & 61.17 & 54.1 \\
\hline 28 & 40.64 & 42.48 & & & & & \\
\hline 29 & 45.28 & 45.31 & 43.05 & 43.88 & 42.07 & 47.83 & 47.81 \\
\hline 30 & 53.42 & 52.94 & 47.11 & 44.57 & 44.93 & 48.14 & 50.1 \\
\hline 31 & 37.76 & 38.68 & 38.69 & 44.87 & 35.11 & 40.92 & \\
\hline 32 & 45.31 & 47.89 & 49.39 & 49.4 & 49.51 & 55.39 & 47.71 \\
\hline 33 & 44.52 & 45.04 & 44.41 & 45.77 & 44.49 & 51.77 & 52.34 \\
\hline
\end{tabular}




\subsubsection{Discussion}

This study has shown that serum periostin levels are influenced by bone injury. A biphasic response is apparent in which the periostin level fell in the first 48 hours of injury, and then progressively increased over the following weeks. The magnitude and duration of this increase in periostin levels was determined by the severity of the bone injury and associated soft tissue damage, with the periostin level increasing almost two-fold eight weeks after joint replacement surgery, and still remaining above presurgery reference levels at 26 weeks. The clinical significance of these findings is that a history of recent bone injury needs to be considered if serum periostin is to be used to predict response to monoclonal antibody therapy in asthma.

It was not hypothesised that there would be a biphasic pattern with the initial drop of serum periostin within 48 hours of the index event, or the long duration of the subsequent progressive increase. However, these findings may be explained by the mechanisms of bone healing. Bone healing is a complex process involving a cascade of events and changes in the expression of several thousand genes. The physiological processes involved in fracture healing occurs in three stages: inflammatory, proliferative and reparative, and remodelling. The inflammatory phase occurs immediately, involving haematoma and granulation tissue formation. It is during this phase where pro-inflammatory molecules such as tumour necrosis factor- $\alpha$ (TNF- $\alpha$ ), interleukin (IL)-1 and IL-6, are secreted promoting angiogenesis and proliferation of osteoblasts and osteoclasts. ${ }^{167}$ The acute inflammatory response peaks within the first 
24 hours and is complete after seven days. The proliferative and reparative phase involves the proliferation and transformation of periosteal cells, production of a cartilagenous callus followed by resorption and replacement with new trabecular bone. During this phase osteogenic molecules, such as BMP-2, up-regulate periostin synthesis within the periosteum and the soft callus. ${ }^{107}$ Murine models report high concentrations of periostin mRNA within immature osteoblasts in periosteal tissues within the first three days of fracture healing. ${ }^{119}$ This creates a periostin-rich environment resulting in the proliferation, differentiation and adhesion of osteoblasts. ${ }^{116}$ The final process is remodelling, occurring from roughly six to eight weeks after a fracture, whereby trabecular bone is resorbed by osteoclasts, and then compact bone is deposited within the resorption pit. During this phase, in mouse models, the periosteum's metabolic activity slows, ${ }^{119}$ resulting in a fall in periostin.

It is possible that the immediate fall in serum periostin is due to the 'mopping up' of periostin as part of the inflammatory response. Alternatively, early periostin isoforms secreted by the periosteum may exert paracrine effects and not be measurable in serum, similar to that observed in cutaneous wound repair, ${ }^{168}$ resulting in lower serum periostin values. The later rise in serum periostin may be due to ongoing up-regulation of periostin synthesis, through the proliferative and reparative phases, resulting in excess production of periostin and its release into the circulation. These phases last for six to eight weeks, consistent with the time course of the peak serum periostin in the joint arthroplasty and long bone fracture groups occurring eight weeks after bone injury, after which the levels gradually reduced during the final remodelling phase. The widespread use of non-steroidal anti-inflammatory (NSAIDs) analgesics intra and post 
operatively, and also after the fractures, may have contributed to the patterns observed. Through inhibition of prostaglandins, NSAIDs impair or delay bone healing and decrease the integrity of the healing bone, ${ }^{169}$ an effect which may contribute to the slow and progressive rise in periostin levels.

There are a number of methodological issues that are relevant to the interpretation of the study findings. Three bone injury groups were studied to enable differing types and magnitudes of bone and associated soft tissue injury to be examined. The contribution of soft tissue injury to the changes in periostin levels may have been significant, as periostin is also secreted by tissue fibroblasts in response to injury. ${ }^{165}$ Whether the bone was weight bearing was also likely to be a contributing factor, as mechanical stress in weight bearing bones is associated with periostin levels. ${ }^{170}$ Although 16 participants fractured more than one bone, each participant was allocated to the group depending on the largest bone fractured, under the assumption that this would have the greatest influence on serum periostin values.

Joint replacement surgery, which was chosen as the model of the greatest magnitude of bone and soft tissue damage, also provided the advantage of allowing a pre-injury periostin measurement to be obtained as a reference baseline. As it was not possible to obtain a pre-fracture sample from those who had sustained a fracture, the original intention was to use the 48 hour periostin level as the reference baseline for the fracture groups, on the assumption that the periostin level would not have changed from pre-injury reference levels so soon after an injury. However the $25 \%$ reduction in periostin level at this time point in the joint replacement group indicated that this may 
not be the case. It was for this reason that a post hoc decision was made to use the 26 week value as the reference measurement in both fracture groups.

This study recruited non-asthmatic participants, to minimise confounding of Type 2 inflammation on serum periostin levels, if there were exacerbations during the study period, which are known to influence periostin levels. ${ }^{171}$ However, these results should be generalisable to asthmatics as there are no systematic differences in serum periostin between asthmatic and non-asthmatic adults. ${ }^{100}$ Although inhaled and systemic steroids may have a modest short term effect on serum periostin levels, they were used in only $5 \%$ and $1 \%$ of participants respectively, and as long term treatment, and so this treatment is unlikely to have influenced the results.

In summary, serum periostin was influenced by bone and soft tissue injury, with a biphasic response, characterised by an initial fall in periostin levels in the first one to two weeks, followed by a progressive increase in serum periostin which peaked at eight weeks, and in the case of bone and soft tissue injury associated with joint replacement surgery, was present for at least 26 weeks. These findings are clinically relevant if a pre-defined cut-point of serum periostin is used to determine treatment eligibility to monoclonal antibody therapy directed against IL-4Ra, IL-13 and IgE in asthma. 


\subsection{Study Two: Change in serum periostin after tooth extraction}

\subsubsection{Introduction}

Periostin is significantly upregulated at pressure sites after experimental tooth movement in rats. However it remains unclear if there is a change in serum levels in humans in response to dental insults. Variations to serum periostin levels after tooth extractions would be an important consideration if serum periostin was to be used as a clinical biomarker in asthma.

\subsubsection{Hypothesis}

Given that serum periostin levels were not significantly affected by short bone fractures, it was hypothesised that it was likely to change following a surgical tooth extraction but not after a simple extraction.

\subsubsection{Objectives}

To determine:

- The magnitude of change in serum periostin following a simple or surgical tooth extraction;

- The difference in magnitude of change between the two groups;

- The time taken for serum periostin to return to pre-operative levels in both groups. 


\subsubsection{Methods}

This cohort study recruited 68 participants aged 18 to 75 years, from the Wellington region who were scheduled to have a dental extraction. Participants were recruited via the Wellington hospital dental department as well as participating private dental surgeries.

Participants were divided into two groups, depending on the type of surgery they received: simple extraction group and surgical extraction group. A simple tooth extraction included any procedure resulting in the removal of the tooth without having to drill into the mandible. A surgical tooth extraction included any surgery which required cutting into bone to remove the tooth. Participants were not recruited if they had conditions expected to affect their serum periostin levels. These included: a Doctor's diagnosis of asthma, chronic bronchitis or COPD; wheeze or the use of respiratory inhalers in the past 12 months; hospital admission, significant surgery (including dental surgery), use of systemic corticosteroids or bone fracture all within three months prior to enrolment. Participants were not recruited if they were pregnant or breastfeeding.

Ethical approval was given by the Central Ethics Committee of New Zealand (13/NTB/197). The trial was prospectively registered with the Australia New Zealand Trials registry (ACTRN12614000152628) and written informed consent was obtained 
from all participants prior to testing. For the full information sheet and study protocol, see Appendices III and IV.

Participants attended the MRINZ outpatient facility over a 12 week period. The enrolment visit occurred up to one week prior to the scheduled extraction date (baseline measurement), followed by visits scheduled for one, two, three, four, six and 12 weeks after the dental extraction. At the initial visit, participants completed a General Health Questionnaire (Appendix IX), which was based on questions from the American Thoracic society (ATS) Division of Lung Diseases-78 Questionnaire (DLD-78) ${ }^{166}$ and measurement of their BMI. Serum periostin was measured at every study visit and was determined using the clinical trial version of the Elecsys ${ }^{\circledast}$ Periostin Immunoassay (Roche Diagnostics, Penzbery, Germany) described previously. ${ }^{115}$

Sample size

The clinically important difference in serum periostin is not known. Therefore the sample size of 68 participants was based on $90 \%$ power to detect a 0.8 standard deviation difference, which can been considered a 'large' effect size difference.

\section{Statistical methods}

The distribution of periostin was right skewed and was logarithm transformed for analysis. The analysis of the differences in periostin measurements was carried out on the logarithm transformed scale. The exponent of a difference in logarithms can be interpreted as the ratio of geometric means. Mixed linear models were used to 
estimate the difference from baseline in logarithm periostin from the measurement, with individual participants as random effects. In a model to assess the effect of extraction type, an initial model with an interaction term was fitted. In the event there was no evidence of an interaction, logarithm periostin averaged over all measurement times was estimated.

SAS version 9.4 was used. 


\subsubsection{Results}

Participant characteristics are described in Table 2.9. 68 participants scheduled for tooth extraction were recruited. However one patient withdrew during the first visit, after enrolment, on finding out a tooth extraction was not necessary and so baseline data was not collected. Three other participants were withdrawn by study investigators after the first visit due to their extractions being cancelled, two patients requested withdrawal from the study after the first visit and two patients were lost to follow up. 
Table 2.9: Summary of participant characteristics in Study Two

\begin{tabular}{|c|c|c|c|c|}
\hline \multirow[b]{3}{*}{ Age $^{1}:$ mean (SD) } & \multicolumn{2}{|c|}{ Simple extraction group } & \multicolumn{2}{|c|}{ Surgical extraction group } \\
\hline & \multicolumn{4}{|c|}{ Continuous variables } \\
\hline & $N=41$ & $44.9(14.6)$ & $N=26$ & $32.7(13.3)$ \\
\hline \multirow[t]{2}{*}{$\mathrm{BMI}^{2}:$ mean (SD) } & $N=40$ & $27.5(5.4)$ & $N=24$ & $26.8(5.3)$ \\
\hline & \multicolumn{4}{|c|}{ Categorical variables } \\
\hline Female (\%) & $N=41$ & $23(56.1)$ & $N=26$ & $19(73.1)$ \\
\hline Atopy ${ }^{3}(\%)$ & $N=41$ & $14(34.2)$ & $N=26$ & $18(69.3)$ \\
\hline Non-smoker (\%) & $N=41$ & $20(49)$ & $N=26$ & $16(62)$ \\
\hline Ethnicity & $N=41$ & & $N=26$ & \\
\hline European (\%) & & 34 (82.9) & & $18(69.2)$ \\
\hline Maori (\%) & & $4(9.8)$ & & $2(7.7)$ \\
\hline Pacific (\%) & & $0(0)$ & & $1(3.9)$ \\
\hline Other (\%) & & $2(4.9)$ & & $1(3.9)$ \\
\hline Steroid medication ${ }^{4}$ & $N=41$ & $5(12.2)$ & $N=26$ & $0(0)$ \\
\hline
\end{tabular}

\begin{abstract}
1 Age: years
2 BMl: $\mathrm{kg} / \mathrm{m} 2$

3 Atopy defined as a history of seasonal rhinoconjunctivitis and/or eczema

4 Participants who took corticosteroid containing medication for any reason during the study period

SD: standard deviation, IQR: interquartile range, BMI: body mass index, ng: nanogram, ml: millilitre.
\end{abstract}


Simple tooth extraction group

There were 41 participants in the simple extraction group. The mean age was 44.9 years, ranging from 20.1 to 72.1 years. The mean BMI was $27.5 \mathrm{~kg} / \mathrm{m}^{2}$. Over half of this group were female (23/41; 56.1\%) and smoked (21/41; 52.2\%). 14 participants gave a history of atopic conditions such as eczema or rhinoconjunctivitis. Five people reported taking a steroid containing medication during the study for eczema, rhinitis and a 'blocked ear'.

Pre-extraction, the mean (SD) serum periostin was $46.4(10.9) \mathrm{ng} / \mathrm{ml}$, ranging from 21.1 to $74.3 \mathrm{ng} / \mathrm{ml}$. Post-extraction, this climbed to a mean (SD) of $48.3(9.4) \mathrm{ng} / \mathrm{ml}$ two weeks later (Figure 2.4). 12 weeks after the tooth extraction, mean (SD) periostin was 47.6 (10.5) ng/ml (Table 2.10). 
Table 2.10: Periostin values for the simple and surgical dental extraction groups

\begin{tabular}{|c|c|c|}
\hline Visit & $\mathbf{N}$ & Periostin mean (SD) \\
\hline \multicolumn{3}{|c|}{ Simple tooth extraction group } \\
\hline 1 week pre-operative (baseline) & 41 & $46.4(10.9)$ \\
\hline Week 1 & 39 & $46.3(10.4)$ \\
\hline Week 2 & 34 & $49.0(10.1)$ \\
\hline Week 3 & 36 & $48.3(10.5)$ \\
\hline Week 4 & 38 & $48.0(10.1)$ \\
\hline Week 6 & 38 & $48.0(10.4)$ \\
\hline Week 12 & 38 & $47.9(10.9)$ \\
\hline \multicolumn{3}{|c|}{ Surgical tooth extraction group } \\
\hline 1 week pre-operative (baseline) & 24 & $48.5(9.9)$ \\
\hline Week 1 & 21 & $44.8(9.5)$ \\
\hline Week 2 & 23 & $47.7(9.0)$ \\
\hline Week 3 & 21 & $48.6(10.2)$ \\
\hline Week 4 & 23 & $47.0(8.4)$ \\
\hline Week 6 & 22 & $47.3(9.1)$ \\
\hline Week 12 & 20 & $45.6(7.7)$ \\
\hline
\end{tabular}


Figure 2.4: Box-plot diagram illustrating serum periostin values by week for those who had a simple tooth extraction

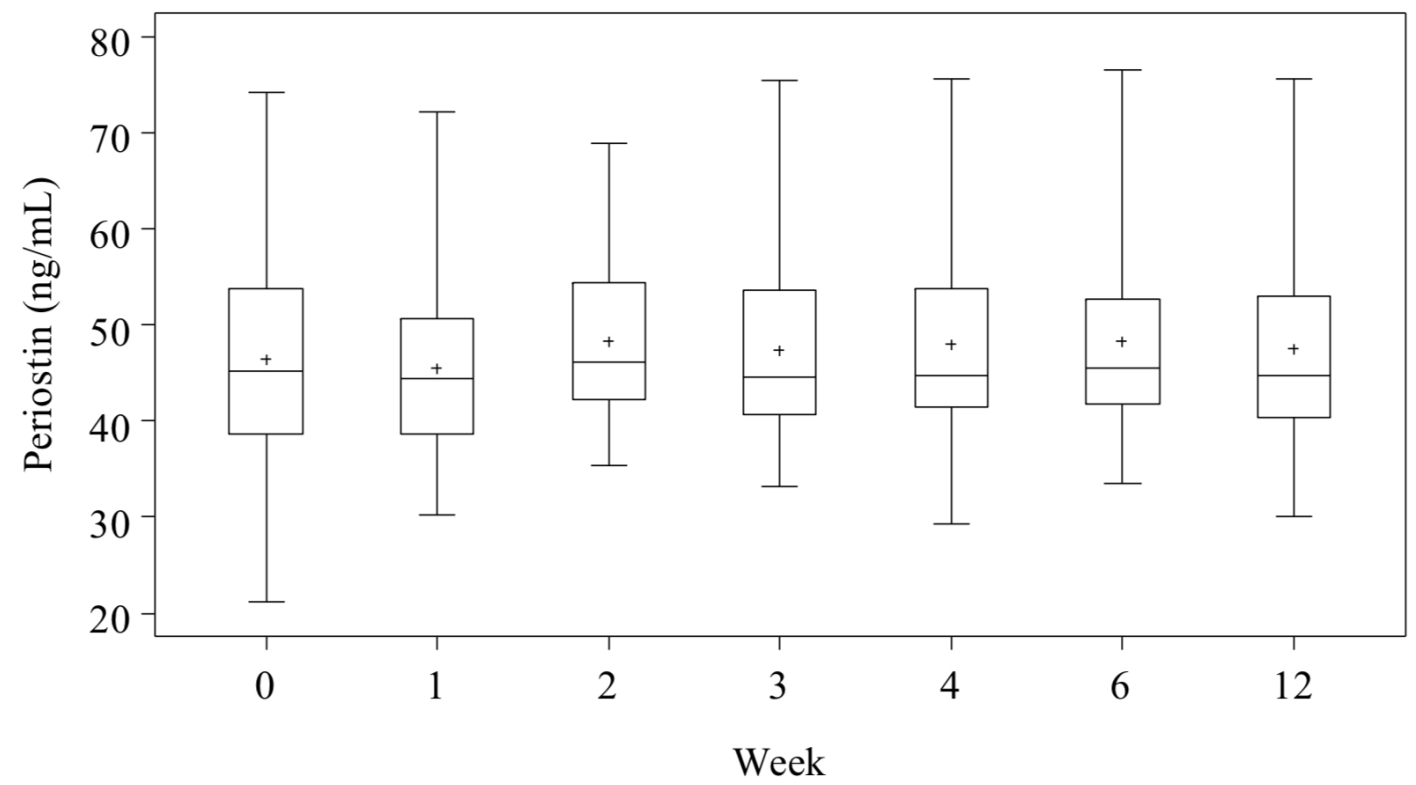


Surgical tooth extraction group

There were 26 participants in the surgical extraction group. The mean age was 32.7 years, ranging from 20.1 to 67.1 years. The mean BMI was $26.8 \mathrm{~kg} / \mathrm{m}^{2}$. Over half of this group were female (19/26; 73.1\%); $10 / 26$ (38.5\%) were current smokers; and no participant reported taking and steroid-containing medication for the duration of the study.

Pre-extraction, the mean (SD) serum periostin was $48.5(9.9) \mathrm{ng} / \mathrm{ml}$, ranging from 34.2 to $68.0 \mathrm{ng} / \mathrm{ml}$. Post-extraction, it peaked to a mean (SD) of $48.6(10.2) \mathrm{ng} / \mathrm{ml}$ at three weeks (Figure 2.5). At 12 weeks after the tooth extraction, the mean (SD) serum periostin was $45.6(7.9) \mathrm{ng} / \mathrm{ml}$ (Table 2.10).

Figure 2.5: Box-plot diagram illustrating serum periostin values by week for those who had a surgical tooth extraction

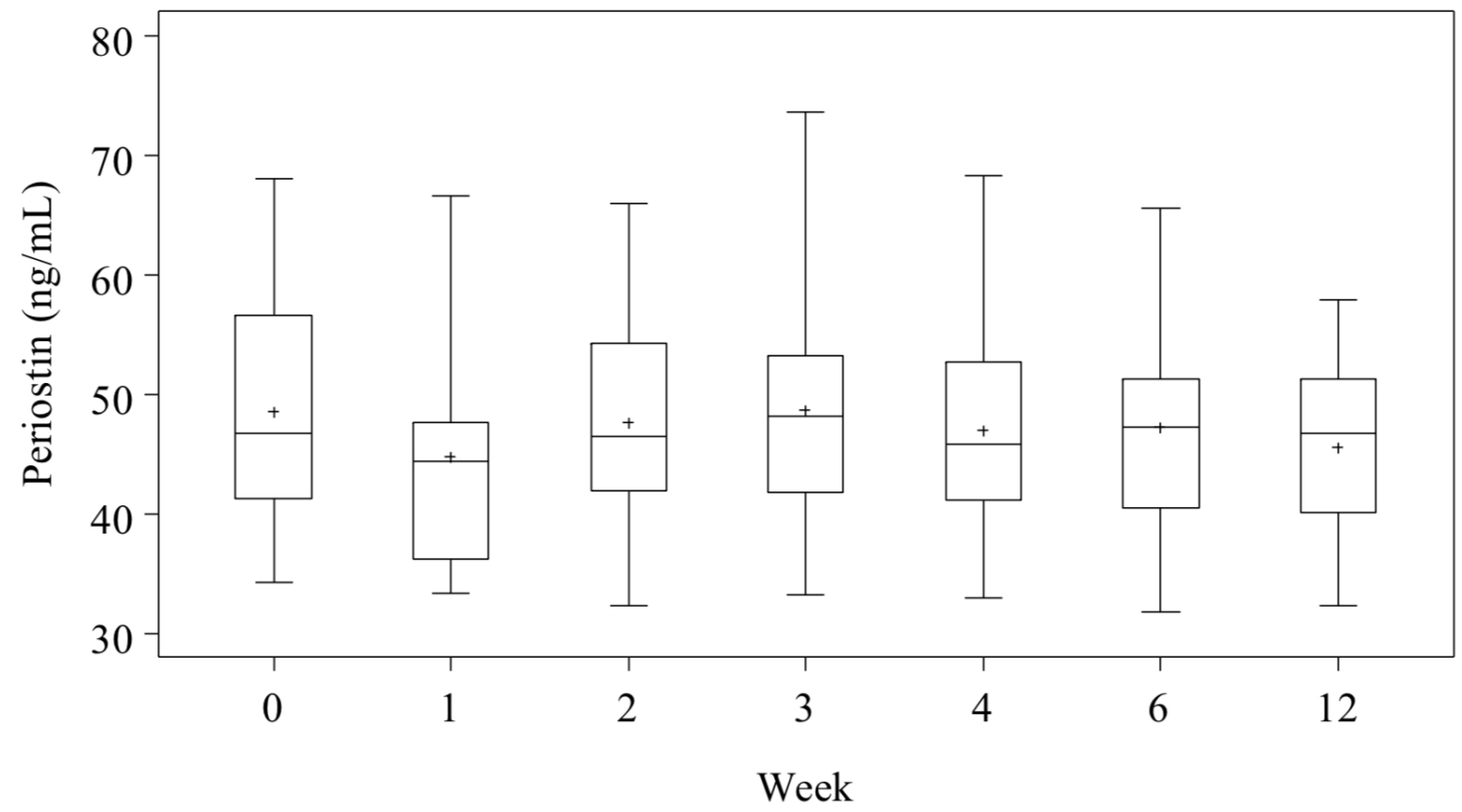


Comparison between surgical and simple extraction groups

Comparison between the two groups revealed no difference in serum periostin, represented by a ratio of geometric mean periostin (95\% Cl) of 1.00 (0.96 to 1.04), $\mathrm{P}=0.85$. Additionally, there was no evidence of an interaction between the type of extraction and time $(P=0.92)$. Given that there was no difference between the surgical extraction group and the simple extraction group, analysis estimating a difference in serum periostin between the weeks was done on all participants (Table 2.11). There was no statistical difference between pre-extraction periostin values and any postextraction measurement, represented by a maximal ratio of geometric mean periostin $(95 \% \mathrm{Cl})$ of $1.02(0.95$ to 1.10$)$, with all with an overall $\mathrm{P}=0.70$.

Table 2.11: Estimates of change of serum periostin from baseline for both groups

\begin{tabular}{lccc}
\hline Visit & Difference in Log Periostin & Ratio of Geometric Mean & P \\
& $(95 \% \mathrm{Cl})$ & Periostin $(95 \% \mathrm{Cl})$ & \\
\hline Week 1 & $-0.036(-0.107$ to 0.035$)$ & $0.96(0.9$ to 1.04$)$ & 0.32 \\
Week 2 & $0.023(-0.049$ to 0.096$)$ & $1.02(0.95$ to 1.10$)$ & 0.53 \\
Week 3 & $0.021(-0.051$ to 0.093$)$ & $1.02(0.95$ to 1.10$)$ & 0.57 \\
Week 4 & $0.017(-0.054$ to 0.088$)$ & $1.02(0.95$ to 1.09$)$ & 0.64 \\
Week 6 & $0.02(-0.051$ to 0.091$)$ & $1.02(0.95$ to 1.10$)$ & 0.58 \\
Week 12 & $0.002(-0.069$ to 0.074$)$ & $1.00(0.93$ to 1.08$)$ & 0.95 \\
\hline
\end{tabular}




\subsubsection{Discussion}

The finding that serum periostin levels were not affected by tooth extractions, regardless of the type of extraction, contrast with those relating to major bone and soft tissue injury, in which a biphasic response is observed (Study One). They suggest that, although periostin is a matricellular protein that responds to mechanical stress and is increased at pressure sites within the PDL, ${ }^{164}$ the magnitude of the local injury is insufficient to cause an increase in the serum periostin levels.

These results may be due, in part, to participants having concurrent periodontal inflammation, which results in down-regulation of periostin expression in the PDL in rat models. ${ }^{172} \mathrm{~A}$ limitation of this study was the fact that a detailed dental history was not taken from subjects at enrolment. However, from these results, it is clear that localised changes to periostin at the PDL in rats are not replicated in serum levels in humans, suggesting that concurrent periodontal inflammation may not have been a significant confounder in this study. Another limitation was that the effect of multiple tooth extractions was not accounted for. However, given the lack of change in serum periostin shown overall, further subdivision of the data would likely result in Type II error from multiple statistical testing. This study was conducted in non-asthmatic adults. However it has previously been demonstrated that serum periostin does not differentiate between asthma and non-asthma, ${ }^{100}$ therefore these findings are applicable to an asthmatic population. Finally, our participants were predominantly New Zealand European and so these results may not be generalisable to other races. 
In conclusion, serum periostin levels did not vary following tooth extraction in nonasthmatic adults, which is noteworthy if it is to be used as a biomarker in predicting responsiveness to monoclonal antibody therapy in asthma. 


\section{Chapter 3: Periostin as a biomarker}

Potential uses of periostin as a biomarker in asthma could be:

- For use as a diagnostic marker in Type 2 asthma;

- To differentiate between asthmatics of varying severity, and;

- To identify potential responders to asthma treatment.

To date, there is conflicting evidence with regards to serum periostin being used as a diagnostic marker in asthma. Study groups in Japan have found that asthmatics have higher mean serum periostin levels than those with no respiratory disease. ${ }^{97,173}$ Furthermore, those with increased serum periostin levels were more likely to have severe disease, on a higher treatment step and suffer with a greater rate of severe exacerbations. ${ }^{97}$ These findings have not been replicated in studies in predominantly Caucasian populations ${ }^{100,101,174}$ who have found that serum periostin was not useful in the diagnosis of asthma, nor could it discriminate between those with more severe forms of the disease from those without. Asthmatics in randomised controlled trials, designed to examine the efficacy of agents directed against the Type 2 pathway, have demonstrably similar mean serum periostin levels regardless of how severe their asthma, or their phenotype. ${ }^{53,69}$ It remains unclear why these findings are so conflicting. 
Serum periostin is currently measured using assays which have yet to be clinically validated. There are multiple assays available, each employing a slightly different monoclonal antibody (or combination of monoclonal antibodies) that target different epitopes of periostin in a sandwich immunoassay either using electrochemiluminescence technology ${ }^{115}$ or an enzyme-lined immunoabsorbent assay (ELISA). ${ }^{16}$ Whilst these tests have been shown to have good reproducibility $(1.7 \%$ to $3.1 \%$ and $1.5 \%$ to $3.3 \%$, respectively), it is not clear if there are differences in their limits of quantification of serum periostin, as there have been no head to head studies comparing the assays. Alternatively, ethnicity may play a role in determining serum periostin level, where those of European descent have lower levels than their East Asian counterparts, regardless of asthma state.

Recently, two large cross-sectional studies reported reference ranges for serum periostin both in an adult group without asthma ${ }^{174}$ and in an adult group with symptomatic airflow obstruction. ${ }^{100}$ In both these studies, participants who identified as being 'Asian' had a trend towards higher serum periostin levels than their Caucasian counterparts. However, the interpretation of these findings was difficult, as in both studies the proportion of people from an Asian background was small, comprising $34 / 480(7 \%)$ and $9 / 386(2 \%)$ of the clinical cohorts, respectively, and the origin of the Asian participants was not further defined. As yet, no studies have investigated whether serum periostin differs between ethnicities.

The intra-participant variability of serum periostin over time would be an important characteristic to define if periostin was to be used as a biomarker in asthma. The other 
Type 2 biomarkers have been well characterised in this sense with blood eosinophils demonstrating diurnal variation, ${ }^{175-179}$ FeNO varying up to $20 \%$ within an individual ${ }^{53}$ and serum IgE levels changing in response to multiple environmental ${ }^{180}$ and seasonal ${ }^{181}$ factors. A desirable feature of periostin would be to have little intra-participant variability, in the absence of unstable asthma.

Periostin has been shown to be a useful marker of identifying responders to monoclonal antibody therapy directed against IL-13, ${ }^{53,54}$ IL4-R $\alpha^{52}$ and IgE. ${ }^{69}$ Those with periostin levels of greater than $50 \mathrm{ng} / \mathrm{ml}$ showed an almost $10 \%$ improvement in their lung function whilst on treatment with anti-IL-1353 and a $30 \%$ reduction in exacerbation rate whilst receiving anti-IgE therapy. ${ }^{69}$ However, given that mean serum periostin levels in a non-asthmatic population is $51.2 \mathrm{ng} / \mathrm{ml},{ }^{174}$ further research needs to be undertaken to identify potential cut-points for initiating treatment with monoclonal antibody therapy.

The main questions being addressed with the following studies are:

- Is serum periostin higher in Chinese adults?

- Does serum periostin vary within an individual over time, whilst their asthma is well controlled?

- Is the currently employed arbitrary cut-point of $50 \mathrm{ng} / \mathrm{ml}$ suitable for initiating monoclonal antibody therapy in asthmatics with poorly controlled asthma? 


\subsection{Study Three: Serum periostin levels in adults of Chinese descent}

\subsubsection{Introduction}

Reference ranges for serum periostin have been defined for non-asthmatic and asthmatic groups in which a trend of higher periostin levels in Asians was observed. The clinical utility of periostin measurements depends on better understanding of factors that may affect serum periostin levels, such as race.

\subsubsection{Hypothesis}

The hypothesis was that serum periostin levels would be significantly higher in Chinese participants that in their Caucasian counterparts.

\subsubsection{Objectives}

To determine:

- A reference range for serum periostin in adult Chinese participants both with and without asthma

- Whether periostin levels differed in Chinese with and without asthma

- Whether country of birth influenced serum periostin levels in the Chinese groups. 


\subsubsection{Methods}

This was a two-centre, cross-sectional study which recruited Chinese adults, aged 18 to 75 years, from the Greater Wellington and Auckland regions. To be eligible for inclusion, participants were required to self-report both their own, as well as their parents', race as Chinese. The study consisted of 188 Chinese participants, divided into non-asthma and asthma groups. For each Chinese group there was a comparator Caucasian group comprising participants who self-reported their race as New Zealand European, derived from previous studies. ${ }^{100,174}$

For both Chinese groups and the Caucasian non-asthmatic group, participants were excluded if they were current smokers, or former smokers with a smoking history of greater than 10 pack years; underwent surgery (including dental surgery), were admitted to hospital, sustained a bone fracture or received systemic corticosteroids within three months of enrolment; were pregnant or breastfeeding; or had an active (within three weeks prior to the study visit) respiratory tract infection, as these scenarios might influence serum periostin levels.

Non-asthmatic group: 120 Chinese participants, without a doctor's diagnosis of asthma or COPD, with at least 20 participants (10 male and 10 female) recruited to each of the following age bands: $18-30,31-45,46-60$ and $61-75$ years. 420 Caucasian adults, without a doctor's diagnosis of asthma or COPD, aged 18-75 years, were derived from 
a previous study identifying reference ranges of periostin in an adult non-asthmatic population. ${ }^{174}$

Asthmatic group: 68 Chinese participants with a doctor's diagnosis of asthma whose current asthma treatment was either of: (i) short-acting beta agonist (SABA) only, or (ii) SABA and at least one controller. 170 Caucasian adults, with a doctor's diagnosis of asthma and aged between 18-75 years were derived from a previous study of an adult population with symptomatic airflow obstruction, ${ }^{100}$ and were stratified as above, based on their current asthma treatment.

Ethical approval was given by the Central Regional Ethics Committee of New Zealand (13/NTB/190). The trial was prospectively registered with Australian New Zealand Clinical Trials Registry (ACTRN12614000122651) and written informed consent was obtained from all participants prior to testing. For the full information sheet and protocol, see Appendicies V and VI. Participants attended the nearest research facility for a single visit for assessment of medical history, completion of a genogram to document race and country of birth, measurement of spirometry and FeNO, and blood sampling for measurement of full blood count (FBC), creatinine and electrolytes, serum IgE and serum periostin. Asthmatic participants answered additional validated respiratory health questionnaires, Asthma Control Questionnaire (ACQ-5) ${ }^{182}$ and Asthma Quality of Life Questionnaire with Standardised Activities (AQLQ-S),, ${ }^{183}$ (Appendices $\mathrm{X}$ and $\mathrm{XI}$ ) to establish their current asthma control. The ACQ-5 is a five point questionnaire, validated by Juniper and group in 2005, after surveying 1323 people (aged 12 years and older) with asthma, and comparing points scored to the 
'gold standard' composite scoring system based on international guidelines defining well controlled and poorly controlled asthma. The psychometric properies of this questionnaire was found to be concordant with the 'gold standard'. Similarly, the same research group proposed the use of the AQLQ in 1996 as a tool for use in clincal trials as a questionnaire measuring quality of life among asthmatics. This was validated among 40 adult asthmatics with symptoms in a nine week observational study in 1996. Both questionnaires are widely used in cinical trials when meauring asthma control and functional activity. Medication history for all participants was recorded.

\section{Spirometry and FeNO}

Spirometry was performed for measurement of $\mathrm{FEV}_{1}$ and FVC using a Masterscreen Pneumo (Masterscreen Version 2.0, Carefusion, Germany) in accordance with the ATS guidelines. ${ }^{184} \mathrm{FEV}_{1}$ percent predicted values were calculated using the Global Lung Initiative equations. ${ }^{185}$ FeNO was assessed using a nitric oxide monitor (NiOX, Aerocrine AB, Sweden) according to ATS guidelines. ${ }^{89}$

\section{Blood samples}

All participants, including from the previous cohorts, underwent venepuncture for measurement of serum periostin, which was determined using the Elecsys ${ }^{\circledast}$ Periostin immunoassay (Roche Diagnostics, Penzberg, Germany) described previously. ${ }^{115}$ Blood samples were coagulated, centrifuged and serum aliquots stored at $-80^{\circ} \mathrm{C}$ prior to analysis. 
FBC and differential (Sysmex platform, Mundelein, USA), urea and electrolytes (Roche, Cobas 501, NZ) and IgE (Roche modular, Indianapolis, USA) were performed immediately in local laboratories.

\section{Study Power}

The sample size of 120 non-asthmatic Chinese adults (60 male; 60 female) was based on the recommendations of the Clinical and Laboratory Standards Institute, ${ }^{186}$ to allow $90 \%$ confidence intervals to be computed by non-parametric methods if normal distribution assumptions were not met. Based on the standard deviation (SD) of logarithm periostin of 0.22 , a sample size of 120 Chinese participants and 420 Caucasian participants had $90 \%$ power with alpha $5 \%$ to detect a difference in mean logarithm periostin of 0.074 which is equivalent to a ratio of mean periostin of 1.08 .

\section{Statistical Methods}

Data descriptions for continuous variables were by mean, median and minimum to maximum ranges. Some of the variables, such as serum IgE, FeNO and serum periostin, with skewed distributions were better analysed on the logarithm transformed scale. For univariate comparison of continuous variables by dichotomous variables, t-tests were used, based on a logarithm transformation when needed. For completeness, for those variables analysed on the logarithm transformed scale, the univariate association by a Mann-Whitney test and Hodges-Lehmann estimator of location shift were also shown. Univariate comparison of categorical variables was by estimation of relative risk. Where a logarithm transformation of a response variable was carried out, 
the exponent of this was shown and was interpreted as the ratio of geometric means. Estimates of the mean and median periostin levels and $90 \%$ confidence intervals for prediction were determined for the Chinese non-asthma group with an analysis of variance (ANOVA). The sex and age adjusted reference range for periostin was estimated by analysis of co-variance (ANCOVA). We performed the analysis on the logarithm transformed scale with a back transformation to establish the $90 \%$ confidence interval for prediction. An estimate of the difference between the Chinese and Caucasian groups was calculated by a general linear model (ANOVA). An exploratory analysis was done to examine the effects of country of birth on serum periostin levels. Finally, ANOVA and ANCOVA were used to examine the association between periostin (using logarithm periostin as the response variable) and the interaction between race and asthma status without (ANOVA) and with (ANCOVA) adjustment of the continuous co-variates $\mathrm{BMI}$ and $\mathrm{FEV}_{1} \%$ predicted.

SAS version 9.4 was used. 


\subsubsection{Results}

The flow of Chinese participants through the study is shown in Figure 3.1. Participants were recruited between May and November 2015 from both sites. A total of 182 people were screened, of which nine were excluded for either not taking medication or declining to participate. 12 participants, who self-identified as Chinese from a previous study ${ }^{174}$ were included in the Chinese non-asthma group, and were part of the final analysis. A total of 185 Chinese participants had complete data, which was analysed and is presented here.

Chinese non-asthma and Caucasian non-asthma groups

Participant characteristics are described in Table 3.1. The Chinese non-asthmatic group had more females $(77 / 118,68 \%$ of total) and a lower mean BMI than the Caucasian group. Atopic conditions, such as seasonal rhinoconjunctivitis or eczema, were less prevalent in the Chinese group, $32.2 \%$, compared to $48.3 \%$ in the Caucasian group. The distribution of serum IgE and FeNO, like periostin, were highly skewed and better analysed on the logarithm transformed scale. Serum IgE was higher in the Chinese non-asthmatic group compared to the Caucasian group. There was no difference between the groups with respect to peripheral blood eosinophils or FeNO. 
Figure 3.1: Flow of Chinese participants through the study

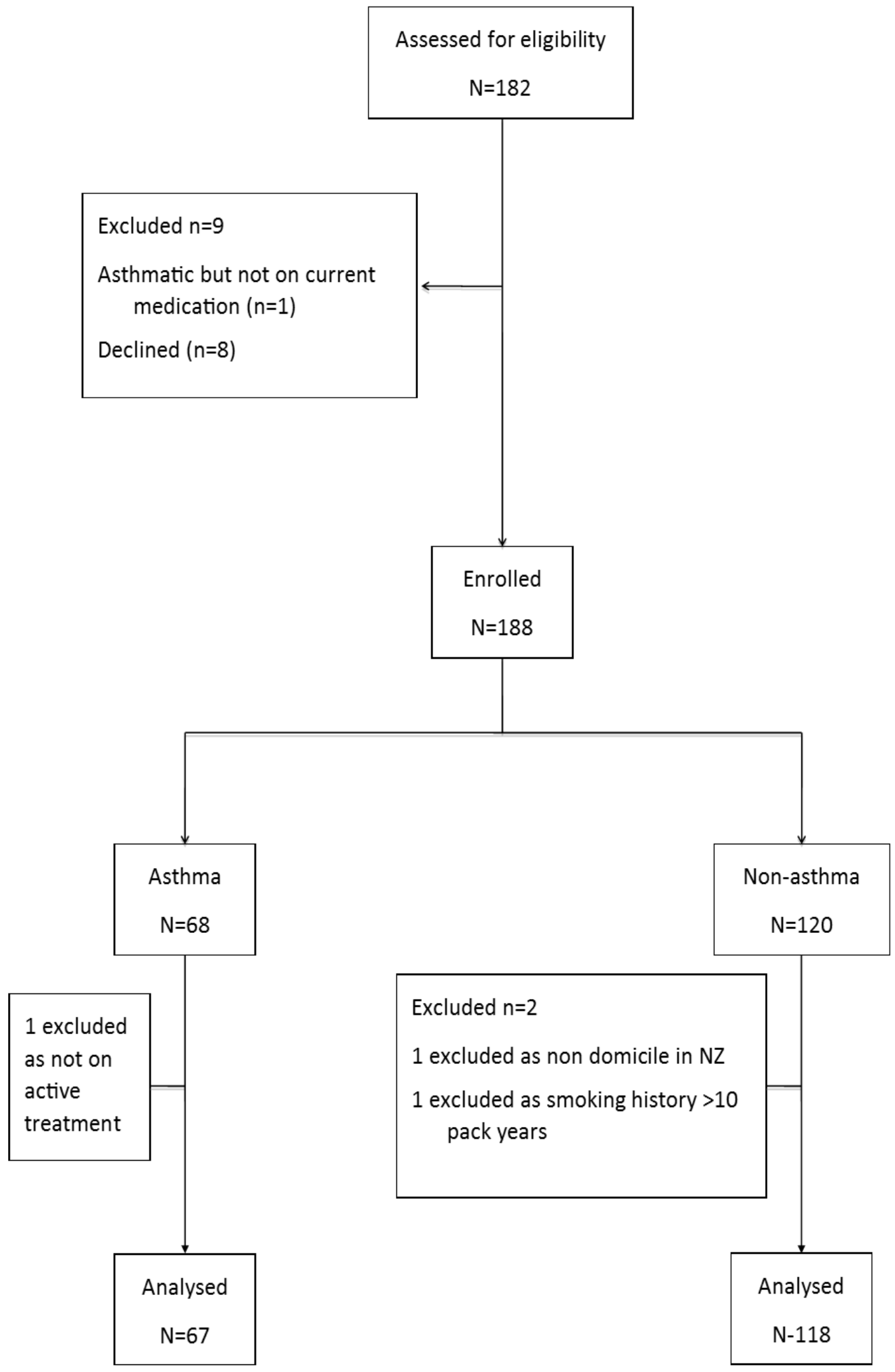


Table 3.1: Participant Characteristics of Non-Asthma groups

\begin{tabular}{|c|c|c|c|c|c|c|c|c|}
\hline \multirow[b]{2}{*}{ VARIABLE } & \multicolumn{3}{|c|}{ CHINESE } & \multicolumn{3}{|c|}{ CAUCASIAN } & \multirow{2}{*}{$\begin{array}{l}\text { Difference } \\
(95 \% \mathrm{Cl})^{*}\end{array}$} & \multirow[b]{2}{*}{$\mathbf{P}$} \\
\hline & $\mathrm{N}$ & Mean (SD) & Min to Max & $\mathrm{N}$ & Mean (SD) & Min to Max & & \\
\hline Age (years) & 118 & $42.2(17.6)$ & 18 to 73 & 420 & $46.4(16.9)$ & 18 to 74 & $-4.3(-7.8$ to -0.8$)$ & 0.017 \\
\hline BMI $\left(\mathrm{kg} / \mathrm{m}^{2}\right)$ & 118 & $23.4(3.5)$ & 17.6 to 33.6 & 420 & $26.1(4.7)$ & 18.2 to 57.5 & $-2.7(-3.6$ to -1.8$)$ & $<0.001$ \\
\hline $\mathrm{FEV}_{1} / \mathrm{FVC}$ ratio $^{\ddagger}$ & 118 & $0.83(0.08)$ & 0.61 to 1.26 & 419 & $0.77(0.07)$ & 0.48 to 0.99 & 0.05 (0.04 to 0.07$)$ & $<0.001$ \\
\hline $\mathrm{FEV}_{1} \%$ & 117 & $104.9(13.7)$ & 64.0 to 135.9 & 419 & $103.7(12.4)$ & 71.3 to 148.5 & $1.25(-1.36$ to 3.85$)$ & 0.35 \\
\hline $\operatorname{Serum}_{\text {periostin }}{ }^{\dagger}(\mathrm{ng} / \mathrm{mL})$ & 118 & $59.6(15.4)$ & 22.1 to 132.1 & 420 & $50.9(12.1)$ & 28.1 to 136.4 & 8.8 (6.1 to 11.4$)$ & $<0.001$ \\
\hline $\mathrm{FeNO}^{\dagger}(\mathrm{ppb})$ & 118 & $28.8(34.2)$ & 5.0 to 300.0 & 420 & $23.3(14.1)$ & 2.5 to 99.0 & 5.5 (1.36 to 9.64$)$ & 0.009 \\
\hline Serum $\operatorname{IgE}^{\dagger}(\mathrm{U} / \mathrm{L})$ & 118 & $270.9(717.8)$ & 2.0 to 5888 & 420 & $94.3(247.3)$ & 0.5 to 2608 & 176.6 (94.7 to 258.6 ) & $<0.001$ \\
\hline Blood eosinophils & 117 & $0.15(0.12)$ & 0 to 0.72 & 419 & $0.16(0.12)$ & 0 to 0.8 & 0.1 (0.1 to 0.2 ) & \\
\hline x10 1 L (units) & & & & & & & & \\
\hline
\end{tabular}

${ }^{*}$ Comparison of variables using t-test: Chinese minus Caucasian means

† Better analysed on the logarithm transformed scale

$\ddagger$ Based on pre-bronchodilator spirometry

Abbreviations: SD: Standard deviation; IQR: Interquartile range; $95 \% \mathrm{Cl}$ : $95 \%$ confidence intervals; BMI: Body mass index

FEV1: Forced expiratory volume in one second; FVC: Forced vital capacity; FeNO: Fractional exhaled nitric oxide; IgE: Immunoglobulin E 
The median (interquartile range; IQR) serum periostin level was higher in Chinese nonasthmatics, 57.0 (50.3 to 67.9) $\mathrm{ng} / \mathrm{ml}$, than in Caucasian non-asthmatics, 49.7 (42.8 to 56.5) $\mathrm{ng} / \mathrm{ml}$. The Hodges-Lehmann estimate $(95 \% \mathrm{Cl}$ ) of the difference was 8.2 (5.8 to 10.6), $P<0.001$. Figure 3.2 shows comparative frequency histograms of logarithm transformed serum periostin in Chinese and Caucasian asthmatics.

In the Chinese non-asthma group, periostin levels were sex-, but not age-dependent, with females having higher periostin levels. Using the mean age in this group (42.2years), the back-transformed individual predicted $(90 \% \mathrm{Cl})$ reference range for periostin in females was $61.1 \mathrm{ng} / \mathrm{ml}(41.6$ to 89.8$) \mathrm{ng} / \mathrm{ml}$ and in males was $53.2 \mathrm{ng} / \mathrm{ml}$ (36.1 to 78.3) $\mathrm{ng} / \mathrm{ml}$. There was an inverse relationship between logarithm serum periostin and $\mathrm{BMI}(\mathrm{r}=-0.28, \mathrm{P}=0.002)$ in the Chinese non-asthma group.

\section{Chinese asthma and Chinese non-asthma groups}

Participant characteristics for asthmatic participants are shown in Table 3.2. The Chinese asthma group had a higher proportion of males, $37 / 67(55 \%)$, and were younger than the Chinese non-asthmatics. A history of atopic conditions was more prevalent in Chinese asthmatics, 48/67 (71.6\%). Mean levels of peripheral blood eosinophils $\left(0.29 \times 10^{9} / \mathrm{L}\right)$, FeNO (64.4ppb) and serum IgE $(537.6 \mathrm{U} / \mathrm{L})$, were higher in Chinese asthmatics than non-asthmatic Chinese $\left(0.15 \times 10^{9} / \mathrm{L}, 28.8 \mathrm{ppb}\right.$ and $270.0 \mathrm{U} / \mathrm{L}$, respectively). There was no significant difference in serum periostin between the Chinese asthma and non-asthma groups, median $56.8 \mathrm{ng} / \mathrm{ml}$ and $57.0 \mathrm{ng} / \mathrm{ml}$ respectively, with a Hodges-Lehmann estimate of $(95 \% \mathrm{Cl})-0.1(-4.2$ to 4.2$), \mathrm{P}=0.94$. 
Figure 3.2: Histograms illustrating the differences between distribution of serum periostin between Chinese $(n=118)$ and Caucasians $(n=420)$ without asthma. Median periostin levels were higher in Chinese non-asthmatics compared to Caucasian nonasthmatics.

A: Chinese frequency histogram

B: Caucasian frequency histogram

$A$

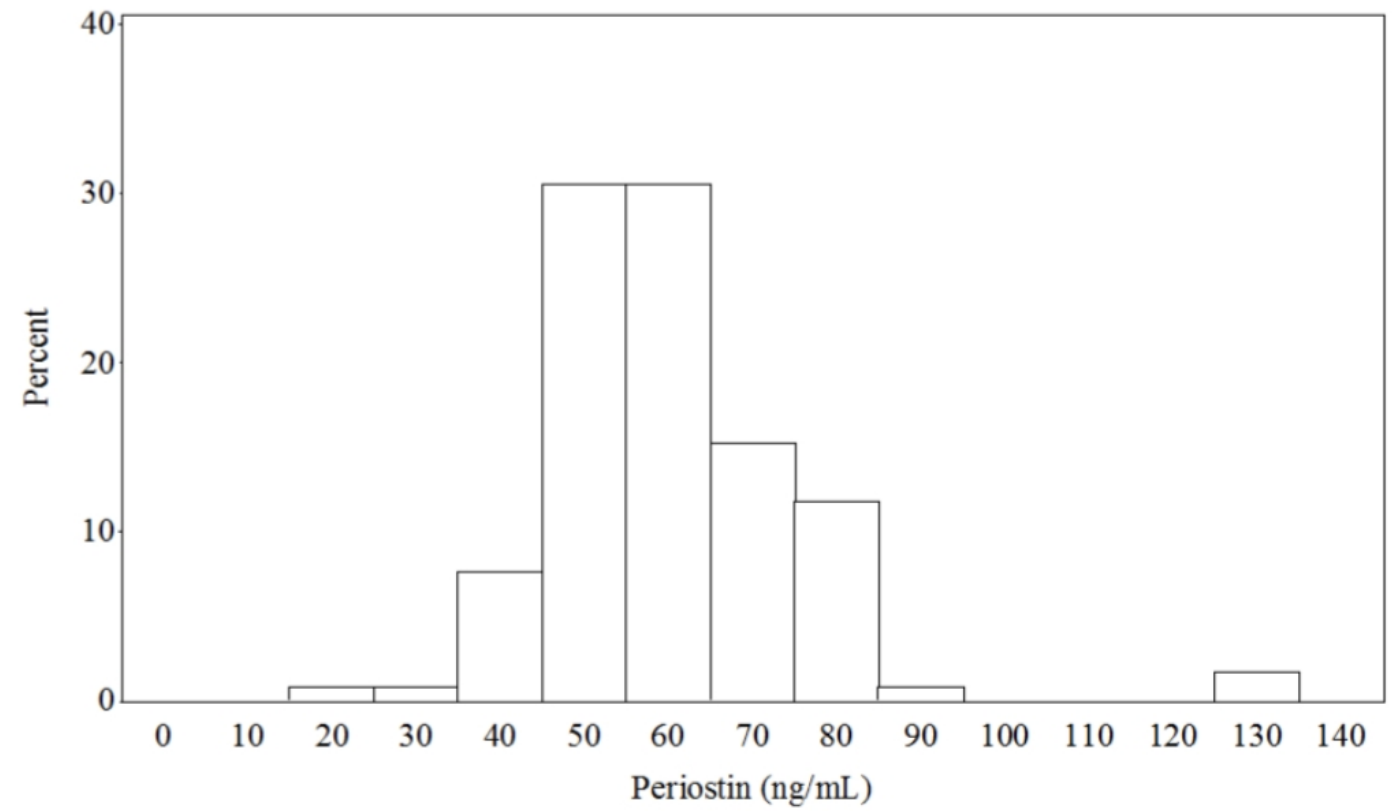

$B$

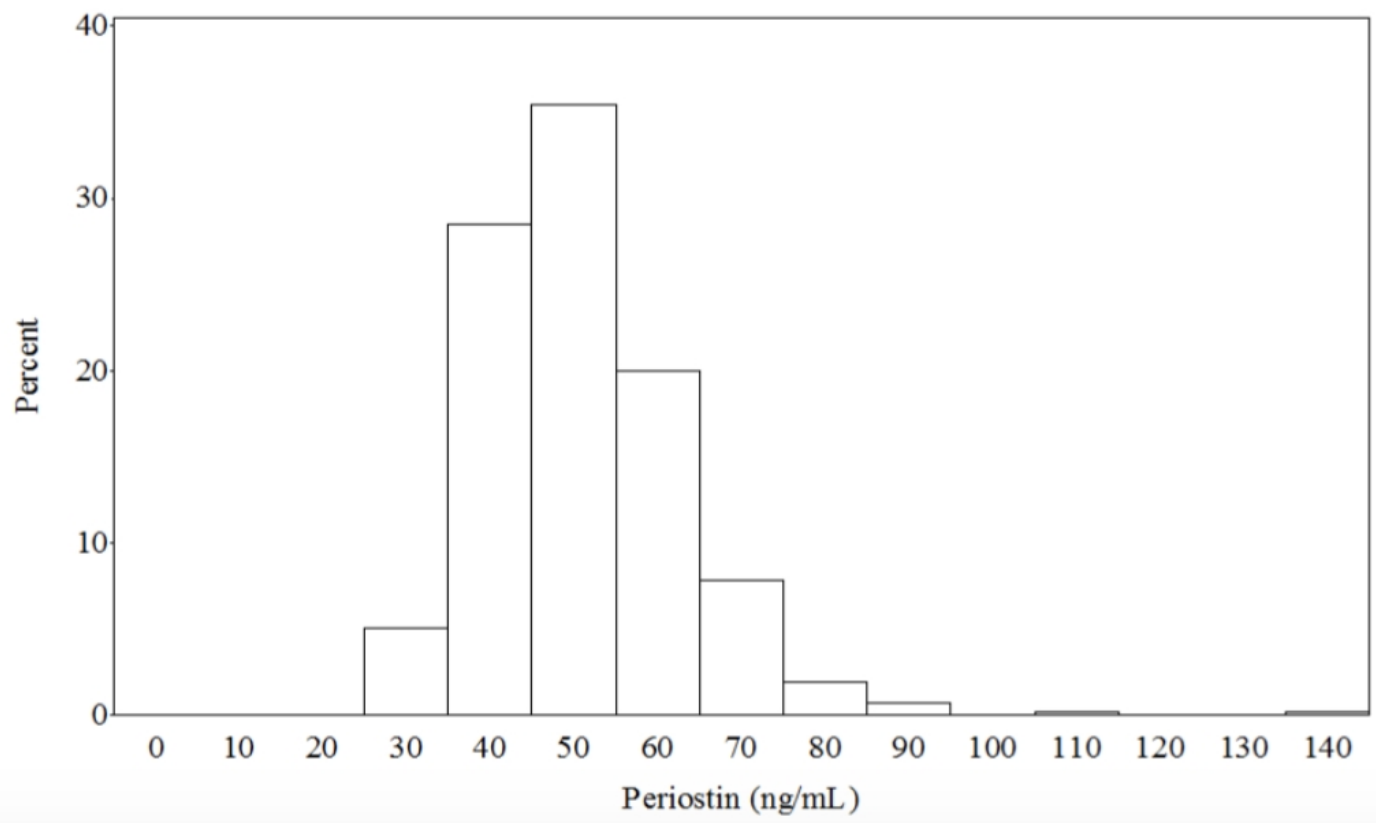


Table 3.2: Participant Characteristics of Asthma Groups

\begin{tabular}{|c|c|c|c|c|c|c|}
\hline \multirow[t]{2}{*}{ VARIABLE } & \multicolumn{3}{|c|}{ CHINESE } & \multicolumn{3}{|c|}{ CAUCASIAN } \\
\hline & $\mathrm{N}$ & $\begin{array}{l}\text { Mean } \\
\text { (SD) }\end{array}$ & $\begin{array}{l}\text { Min to } \\
\text { Max }\end{array}$ & $\mathrm{N}$ & Mean (SD) & $\begin{array}{l}\text { Min to } \\
\text { Max }\end{array}$ \\
\hline Age (years) $N=67$ & 67 & $\begin{array}{l}37.3 \\
(16.3)\end{array}$ & $\begin{array}{l}18.4 \text { to } \\
72.4\end{array}$ & 170 & $46.0(14.5)$ & 19 to 75 \\
\hline BMI $\left(\mathrm{kg} / \mathrm{m}^{2}\right)$ & 67 & $\begin{array}{l}24.9 \\
(3.9)\end{array}$ & $\begin{array}{l}17.6 \text { to } \\
37.4\end{array}$ & 170 & $27.7(6.7)$ & $\begin{array}{l}15.7 \text { to } \\
57.1\end{array}$ \\
\hline $\mathrm{FEV}_{1} / \mathrm{FVC}$ ratio* & 67 & $\begin{array}{l}0.74 \\
(0.11)\end{array}$ & $\begin{array}{c}0.43 \text { to } \\
0.96\end{array}$ & 170 & $0.72(0.11)$ & $\begin{array}{c}0.38 \text { to } \\
0.95\end{array}$ \\
\hline $\mathrm{FEV}_{1} \%$ predicted & 67 & $\begin{array}{l}95.3 \\
(16.6)\end{array}$ & $\begin{array}{l}50.1 \text { to } \\
130.5\end{array}$ & 170 & $83.1(16.3)$ & $\begin{array}{l}32.6 \text { to } \\
121.8\end{array}$ \\
\hline $\begin{array}{l}\text { Serum } \\
(\mathrm{ng} / \mathrm{mL})\end{array}$ & 67 & $\begin{array}{l}59.9 \\
(15.3)\end{array}$ & $\begin{array}{l}36.4 \text { to } \\
128.2\end{array}$ & 170 & $58.9(19.9)$ & $\begin{array}{l}15.0 \text { to } \\
148\end{array}$ \\
\hline $\mathrm{FeNO}^{\dagger}(\mathrm{ppb})$ & 67 & $\begin{array}{r}64.4 \\
(54.8)\end{array}$ & $\begin{array}{l}7.0 \text { to } \\
242\end{array}$ & 170 & $39.3(33.2)$ & $\begin{array}{l}2.7 \text { to } \\
194.1\end{array}$ \\
\hline Serum $\operatorname{IgE}^{\dagger}(\mathrm{U} / \mathrm{L})$ & 66 & $\begin{array}{l}537.6 \\
(632.9)\end{array}$ & $\begin{array}{l}7.0 \text { to } \\
3454\end{array}$ & 170 & $\begin{array}{c}372.8 \\
(1429.0)\end{array}$ & $\begin{array}{c}1 \text { to } \\
18083\end{array}$ \\
\hline $\begin{array}{l}\text { Blood eosinophils } x \\
10^{9} / L \text { (units) }\end{array}$ & 67 & 0.29 & $\begin{array}{l}0.02 \text { to } \\
0.78\end{array}$ & 170 & $0.27(0.22)$ & 0 to 1.5 \\
\hline
\end{tabular}

*Based on pre-bronchodilator measurements

† Better analysed on the logarithm transformed scale

Abbreviations: SD: Standard deviation; IQR: Interquartile range; $95 \% \mathrm{Cl}$ : 95\% confidence intervals; BMI: Body mass index;

FEV1: Forced expiratory volume in one second; FVC: Forced vital capacity; FeNO: Fractional exhaled nitric oxide; IgE:

Immunoglobulin E 
Of the 185 Chinese participants with periostin data, 93 participants were born in New Zealand and 92 participants were immigrants to New Zealand. All participants had lived in New Zealand for at least one year prior to enrolment into the study. As there was no difference in serum periostin between the Chinese asthma and non-asthma groups, this analysis was performed on all Chinese participants, irrespective of their asthma status. There was no difference in periostin levels between those who were born in New Zealand and those born elsewhere, with a Hodges-Lehmann estimate (95\% Cl) of -1.3 (-5.1 to 2.6), $\mathrm{P}=0.51$, (Figure 3.3).

Figure 3.3: Box-plot of serum periostin by country of birth for both non-asthmatic and asthmatic Chinese participants $(n=185)$. The symbol is the mean, the horizontal lines are the 25th percentile, median and 75th percentile, and the whiskers extend from the minimum to maximum values. There was no difference in serum periostin levels between Chinese born in New Zealand versus Chinese born elsewhere.

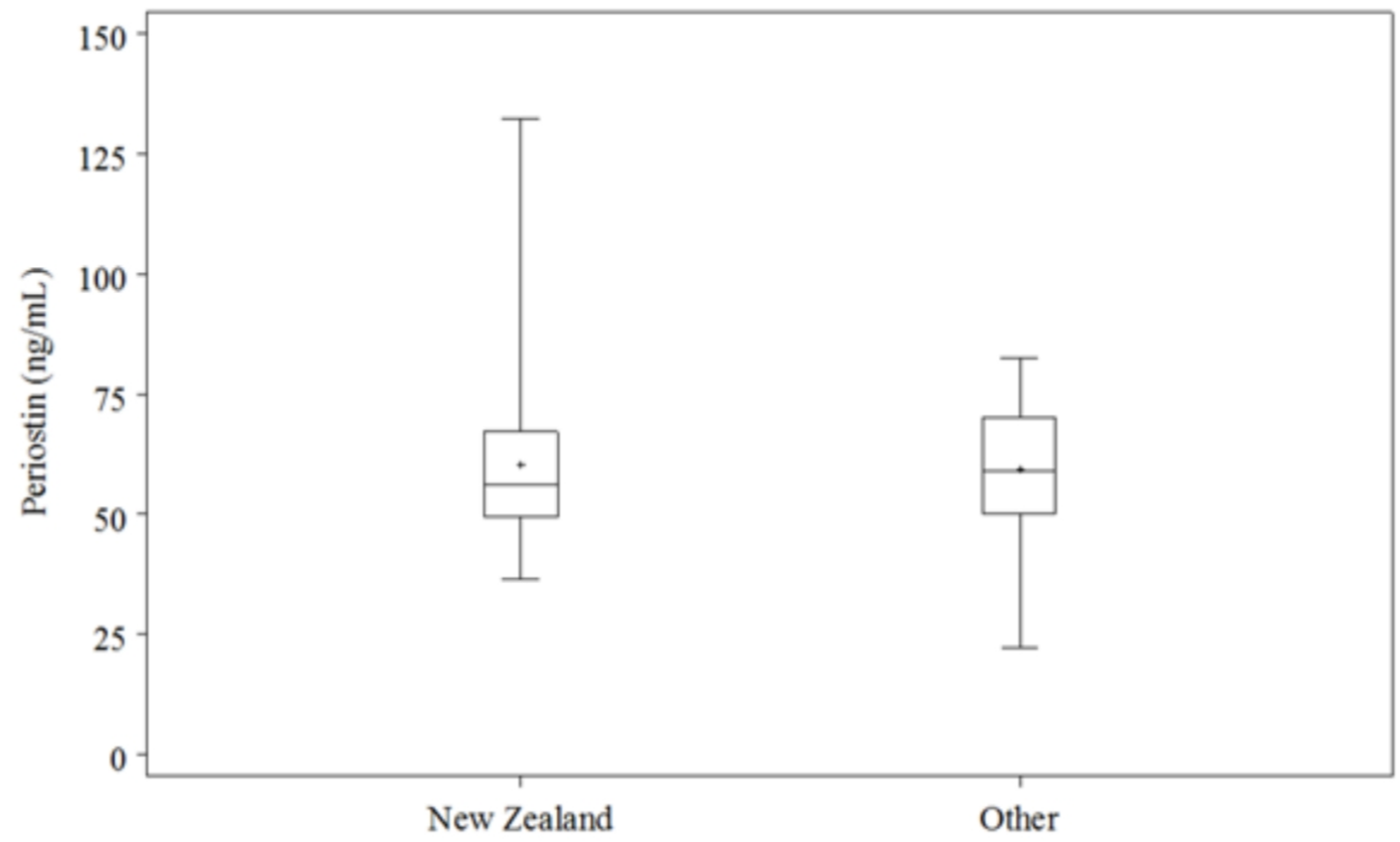


Chinese asthma and Caucasian asthma groups

The Chinese asthma group had a lower BMI than the Caucasian group and a higher mean $\mathrm{FEV}_{1} / \mathrm{FVC}$ ratio and higher $\mathrm{FEV}_{1} \%$ predicted values. Spirometric differences were consistent with a lower proportion of Chinese participants being on Global Initiative for Asthma (GINA) treatment Step 2 or higher; 33/67 (49\%) in the Chinese group and $106 / 170(62 \%)$ in the Caucasian group. There was evidence of modest interaction between race and asthma status on serum periostin levels with an unadjusted value $\mathrm{P}=0.01$, and a value of $\mathrm{P}=0.024$ after adjustment for $\mathrm{BMI}$ and $\mathrm{FEV}_{1} \%$ predicted.

With respect to biomarkers of Type 2 asthma, median serum periostin levels between the two groups were similar $(56.8 \mathrm{ng} / \mathrm{ml}$ in Chinese and $54.9 \mathrm{ng} / \mathrm{ml}$ in Caucasians). Blood eosinophils were similar with means (SD) of $0.29(0.19) \times 10^{\%} / \mathrm{L}$ and $0.27(0.22)$ $\mathrm{x} 10^{\circ} / \mathrm{L}$ in Chinese and Caucasian asthmatics. However, FeNO and IgE were higher in Chinese asthmatics with a mean (SD) FeNO of 64.4 (54.8) ppb and IgE 537.6 (632.9) U/L compared to 39.3 (33.2) ppb and 372.8 (1429) U/L in Caucasian asthmatics. 


\subsubsection{Discussion}

The main findings of this study were that there was no difference in periostin levels between Chinese adults with and without asthma and that serum periostin levels were higher in non-asthmatic Chinese compared to non-asthmatic Caucasians.

Serum periostin levels were similar in the Chinese asthma and non-asthma groups with median values $56.8 \mathrm{ng} / \mathrm{ml}$ and $57.0 \mathrm{ng} / \mathrm{ml}$ respectively. This finding is in agreement with previous studies undertaken in predominantly Caucasian participants ${ }^{100,174}$ where periostin could not discriminate between asthmatic and non-asthmatic groups, suggesting that measurement of periostin is not useful in establishing a diagnosis of asthma.

Serum periostin levels were higher in Chinese, irrespective of the participant's country of birth. This finding is consistent with previous studies ${ }^{100,174,187}$ which have found higher serum periostin levels in those of Asian origin. Together with studies that described polymorphisms of the POSTN gene influencing serum periostin levels, ${ }^{99}$ this suggests that genetic background plays a key role in determining levels of serum periostin. The clinical relevance is that race may be an important factor to consider when interpreting serum periostin values.

In the Chinese non-asthma population periostin levels were sex-dependent, with females having higher periostin levels. This was an unexpected finding as it was not 
observed in the previous study of a predominantly Caucasian population without asthma, ${ }^{174}$ which was a larger study defining a reference range for non-asthmatic adults. It is possible that differences in key environmental or personal characteristics that influence serum periostin levels may differ between Chinese and Caucasian people, based on sex. However, the most likely explanation for this difference is chance. To clarify the uncertainty, further robust data describing sex-dependent reference ranges in Chinese and other populations defined by racial background will be useful.

The observation that serum periostin was similar between the Chinese and Caucasian asthma groups is difficult to interpret given that the two groups were recruited using different methodology. The Caucasian asthmatics were recruited from the electoral roll and were not excluded if they underwent surgery or dental procedures, or sustained bone fractures prior to enrolment and had less severe asthma, as periostin was not the main focus of this study. ${ }^{100}$ Consequently, formal statistical analysis was not performed between these asthma groups, as any meaningful conclusion would be difficult to interpret given the different approaches to recruitment between the two groups of participants. However, the serum periostin was processed in the same way, utilising the same assay. ${ }^{115}$

The finding that different patterns for other Type 2 biomarkers between the groups were observed supports previous observations that these biomarkers may identify different aspects of Type 2 mediated inflammation. ${ }^{174,188-190}$ Between non-asthmatics, the periostin and serum IgE were higher in Chinese compared with Caucasians, 
whereas there was no significant difference in FeNO or blood eosinophils. In the Chinese population those with asthma had higher levels of FeNO, IgE and blood eosinophils, but not periostin, when compared with those without asthma.

The observation of a higher serum IgE in Chinese compared with Caucasian populations is consistent with previous findings identifying differing serum IgE levels between races and socio-economic groups ${ }^{191-193}$ and specifically a trend towards higher serum IgE in Chinese compared with 'White' people in England. ${ }^{193}$ Smoking, which is associated with elevated levels of serum $\operatorname{IgE}^{194}$ was not a confounding factor in our study as current smokers, or former smokers with a pack year history of over ten years, were excluded. Whilst IgE is a marker of atopy, there are also likely to be genetic factors affecting the expression of serum IgE which may explain the differences seen between races. The finding that blood eosinophil levels were similar in Chinese and Caucasians without asthma is consistent with the previous observation that blood eosinophil levels are comparable between ethnicities, including 'Orientals' (defined as those who were from South East Asia, or who identified as Chinese). ${ }^{195}$ Our finding of similar FeNO levels between Chinese and Caucasians without asthma adds to the literature of inconsistent findings between FeNO and race. ${ }^{196-199}$ Although some groups have reported significantly elevated FeNO in Asians versus their Caucasian peers in both asthmatic ${ }^{197}$ and non-asthmatic ${ }^{199}$ children, the National Health and Nutrition Examination Survey (NHANES) cohort ${ }^{196}$ found only a difference between races in children, but not in adults, in a population free of respiratory diagnoses. In summary, each Type 2 biomarker had different patters between races, which highlights inherent difficulties with surrogate biomarkers, in that they may reflect an inflammatory 
process, but they are also subject to influence by other factors such as race and environment. In this study, race may play a more important role with level of Type 2 biomarkers than asthma status.

Some of the associations we have found should be interpreted cautiously. As this was an exploratory study investigating a potential difference in serum periostin levels between Chinese and Caucasian populations, performing multiple statistical tests may have resulted in Type I error inflation. Secondly, we recruited people who self-reported their race as Chinese. To mitigate against issues around race identity, participants completed a genogram in which both parents were required to identify as Chinese. Thirdly all participants were resident in New Zealand for at least a year prior to enrolment, in order to minimise potential confounding of environmental factors, such as indoor or outdoor air pollution, on serum periostin levels. Consequently, these results may not be generalisable to Chinese people domiciled outside of New Zealand. Despite data from different participant cohorts being used in this study, serum for periostin levels were processed and stored using the same methodology as described previously. ${ }^{174}$ In addition, periostin was measured using the same assay in a central laboratory for all participants. ${ }^{115}$ The inter- and intra-assay coefficients (CVs) range from 1.5 to $3 \%$ and 2.8 to $5.3 \%$, respectively across a broad range of periostin concentrations in human serum. ${ }^{101}$ Finally, the Chinese asthmatics had less severe asthma as measured by $\mathrm{FEV}_{1} \%$ predicted and asthma medication use, although this is unlikely to be of significance, as serum periostin levels are not related to asthma severity. ${ }^{100,101}$ 
In conclusion, serum periostin levels are higher in a Chinese non-asthmatic population compared with a Caucasian non-asthmatic population, suggesting that genetic background may influence serum periostin levels. Further study is recommended to determine serum periostin reference ranges in different racial groups, and whether or not cut-points, which determine responsiveness to monoclonal antibody therapy against IL-13, IL-4Ra and IgE, should be adjusted to take race into account. 


\subsection{Study Four: Longitudinal variation of serum periostin in adults with stable asthma}

\subsubsection{Introduction}

A good biomarker has minimal variability when the biomarker-related disease is stable. Serum periostin is a biomarker of responsiveness to monoclonal antibody therapy directed against IL-13, IL-4R $\alpha$ and IgE in asthma. A characteristic that needs to be established, if periostin is to be used as a biomarker in asthma, is its day-to-day variability over prolonged periods of time in patients with stable asthma.

\subsubsection{Hypothesis}

It was hypothesised that, in the absence of an asthma exacerbation, there would be little intra-participant variation in periostin levels.

\subsubsection{Objectives}

To determine:

- The variation in serum periostin levels in adults with stable asthma on longterm ICS and LABA therapy;

- The possibility of seasonal variation of periostin, and; 
- The proportion of participants who changed between the classification of 'high' periostin and 'low' periostin during the course of the study, using the cut-point of $50 \mathrm{ng} / \mathrm{ml}$.

\subsubsection{Methods}

This prospective cohort study recruited 60 adults aged between 18 and 75 years from the MRINZ volunteer database and local advertising in the Wellington area. Inclusion criteria were a doctor's diagnosis of asthma and prescription of maintenance ICS and LABA therapy at a stable dose for a minimum of three months. Exclusion criteria included scenarios that might reasonably be expected to influence periostin levels, such as unstable asthma (as determined by the investigator and/or a change in asthma medication within the preceding three months), systemic corticosteroid use or hospital admission within three months, major surgery requiring general anaesthetic, tooth extractions or bone fracture occurring within the preceding three months, active (current, or within the preceding three weeks) upper or lower respiratory tract infection, known pregnancy, significant co-morbidity, or, any safety concern at the investigator's discretion.

Ethical approval was given by the New Zealand Health and Disability Ethics Committee (13/NTB/185). The trial was prospectively registered with Australia New Zealand Trial Registry (ACTRN126140000235606) and written informed consent was obtained from 
all participants prior to any study procedures being undertaken. For the full information sheet and study protocol, see Appendicies VII and VIII.

Following telephone pre-screening to assess eligibility to participate in the study, participants attended the MRINZ outpatient facility for a total of 11 visits over 59 days. There were daily visits for the first five days, followed by a sixth visit at day 10 , then weekly visits for four weeks, and a final visit three weeks later. Study procedures were carried out as detailed in the Study Plan (Table 3.3). The recruitment period was over an eleven month period from $10^{\text {th }}$ March 2014 to $16^{\text {th }}$ Feburary 2015.

\section{Questionnaires}

Three health questionnaires were administered (see Appendices IX, X and XI):

i. A General Health Questionnaire was used to ascertain the current health status of participants, implementing questions from the ATS Division of Lung Diseasees-78 Questionnaire (DLD-78). ${ }^{166}$

ii. The AQLQ (S), a validated 32 question self-administered questionnaire that assessed quality of life in the two weeks before the first visit. ${ }^{183}$

iii. The ACQ-5, to determine asthma severity in the week prior to Visit $1 .{ }^{182}$

\section{Lung function and FeNO}

Spirometry was performed with a Masterscreen Pneumo device (Masterscreen Version 2·0, Carefusion, Hoechberg, Germany) in accordance with ATS guidelines. ${ }^{184}$ FeNO was 
measured using a nitric oxide monitor (NIOX MINO; Aerocrine AB, Solna, Sweden) according to ATS guidelines. ${ }^{89}$

Blood samples

Full blood count, with white blood cell differential, and serum IgE (Sysmex platform, Mundelein, USA) were processed immediately. The remaining blood samples were centrifuged and serum aliquots were stored at $-80^{\circ} \mathrm{C}$, prior to analysis of serum periostin. Serum periostin levels were determined using the clinical trial version of the Elecsys $^{\circledast}$ Periostin Immunoassay (Roche Diagnostics, Penzbery, Germany). This assay is an automated electrochemiluminescence immunoassay, based on the sandwich principle utilising the same antibodies reported by Jia and colleagues. ${ }^{98}$

Medication use during the study period

All participants were instructed to continue taking their asthma medication as usual for the duration of the study period. The use of any additional medication or a change to regular medication during the study period was recorded.

\section{Recording exacerbations}

In the event participants suffered with a severe exacerbation, requring systemic corticosteroids, medication changes were recorded at the next clinical visit with the following details recorded: date started, dose taken, duration of prescription. 
Sample size

The sample size of 60 participants was chosen to ensure that even if there was $20 \%$ attrition, 50 participants would remain in the study which would give high precision for the estimate of variances.

\section{Statistical Methods}

As with previous work with periostin, its distribution was right skewed and better treated on the logarithm scale for analysis. ${ }^{100,174,200}$ However, in this group of participants the distribution was not particularly skewed and therefore analyses for limits of agreement were shown both on the raw and logarithm transformed scale. The exploration of differences between month, season, and exacerbation status was performed on the raw scale. Differences in logarithms, where these were presented, were equivalent with exponentiation, to the ratio of geometric means.

For illustrative purposes Bland-Altman limits of agreement plots were shown for differences from baseline however a mixed linear model was used to estimate the variance components for the between and within participant variation to use all the information from the repeated measurements. In these models an exponential power correlation structure was used for the variance-covariance matrix. The intra-class correlation coefficient (ICC) is the ratio of the between participant variance to the sum of the between and within participant variances. A number close to one means most of the variation is between participants. The $95 \%$ prediction limits for an individual value was plus or minus the within participant standard deviation times 1.96 and the $90 \%$ 
limits plus or minus 1.69 times the within participant standard deviation. In order to compare these data with that summarised from previous research where a coefficient of variation was used to illustrate measurement variability, a coefficient of variation (standard deviation divided by the mean expressed as a percentage) was calculated for each participant and the mean coefficient of variation and a $95 \%$ confidence interval for this was estimated, based on a one sample t-test.

Mixed linear models as described above were used to compare months and seasons, treating these as fixed effects. The model-based, least squares means, for each month and season, were also shown as these take into account the repeated measures.

A post hoc analysis estimated the change in periostin around the time of a severe asthma exacerbation, which was defined as worsening asthma symptoms requiring systemic corticosteroids. There were five participants who had a severe exacerbation. For the comparison of periostin before, during (defined as whilst on systemic corticosteroids), and after an exacerbation, the mixed linear model described above was used with fixed effects for whether the measurement was made under one of the exacerbation conditions. The overall $\mathrm{P}$ value for these analyses examined any evidence of a difference between the three time periods in relation to exacerbation. The comparisons shown were for: in exacerbation, defined as using systemic corticosteroids, compared to pre-exacerbation, and after systemic corticosteroids were no longer used, compared to pre-exacerbation. 
For the cut-point analysis the paired proportions of participants above or below a serum periostin level of $50 \mathrm{ng} / \mathrm{ml}$ were compared using McNemar's test and an appropriate estimate of the difference in marginal proportions for the paired contingency table.

SAS version $9 \cdot 3$ was used. 
Table 3.3: Study plan for Study Four

\begin{tabular}{|c|c|c|c|c|c|c|c|c|c|c|c|c|}
\hline Visit No. & $1 \mathrm{a}$ & $1 \mathrm{~b}$ & 2 & 3 & 4 & 5 & 6 & 7 & 8 & 9 & 10 & 11 \\
\hline Time Point & $\begin{array}{l}\text { Up to } 14 \\
\text { days pre- } \\
\text { Day } 1\end{array}$ & $\begin{array}{l}\text { Day } 1 \\
+/-1 \\
\text { day }\end{array}$ & $\begin{array}{l}\text { Day } 2 \\
+/-1 \\
\text { day }\end{array}$ & $\begin{array}{l}\text { Day } 3 \\
+/-1 \\
\text { day }\end{array}$ & $\begin{array}{l}\text { Day } 4 \\
+/-1 \\
\text { day }\end{array}$ & $\begin{array}{l}\text { Day } 5 \\
+/-1 \\
\text { day }\end{array}$ & $\begin{array}{l}\text { Day } 10 \\
+/-1 \\
\text { day }\end{array}$ & $\begin{array}{l}\text { Day } 17 \\
+/-1 \\
\text { day }\end{array}$ & $\begin{array}{l}\text { Day } 24 \\
+/-1 \\
\text { day }\end{array}$ & $\begin{array}{l}\text { Day } 31 \\
+/-1 \\
\text { day }\end{array}$ & $\begin{array}{l}\text { Day } 38 \\
+/-1 \\
\text { day }\end{array}$ & $\begin{array}{l}\text { Day } 59 \\
+/-1 \\
\text { day }\end{array}$ \\
\hline Written Informed Consent & $x$ & & & & & & & & & & & \\
\hline Enrolment & & $x$ & & & & & & & & & & \\
\hline Measurement of BMI & & $x$ & & & & & & & & & & \\
\hline $\begin{array}{l}\text { General Health } \\
\text { Questionnaire }\end{array}$ & & $x$ & & & & & & & & & & \\
\hline ACQ-5 & & $x$ & & & & & $\mathrm{x}$ & $x$ & $x$ & $x$ & $\mathrm{x}$ & $x$ \\
\hline AQLQ & & $x$ & & & & & & & & & & \\
\hline Periostin sample & & $x$ & $x$ & $x$ & $x$ & $x$ & $x$ & $x$ & $\mathrm{x}$ & $x$ & $x$ & $x$ \\
\hline FBC sample & & $x$ & $x$ & $x$ & $x$ & $x$ & $x$ & $x$ & & & & \\
\hline IgE sample & & $x$ & & & & & & & & & & \\
\hline FeNO & & $x$ & & & & & & & & & & \\
\hline Spirometry (FVC + FEV 1 ) & & $x$ & & & & & & & & & & \\
\hline
\end{tabular}

ACQ-5: Asthma Control Questionnaire; AQLQ: Asthma Quality of Life Questionnaire; FBC: full blood count; IgE: immunoglobulin E; FeNO: Fractional exhaled nitric oxide; FVC: forced vital capacity; FEV ${ }_{1}$ : Forced expiratory volume in 1 second 


\subsubsection{Results}

\section{Characteristics of participants}

A total of 60 participants were recruited and the flow of participants in the study is shown in Figure 3.4. Baseline characteristics are shown in Table 3.4. Participants were taking a median ICS dose of 500mcg fluticasone propionate-equivalent per day, together with LABA therapy, and had well-controlled asthma with a mean $\mathrm{FEV}_{1}$ 101\% predicted, a mean ACQ score of 0.89 and, a mean FeNO 24.6ppb. 
Figure 3.4: Patient flow through Study Four

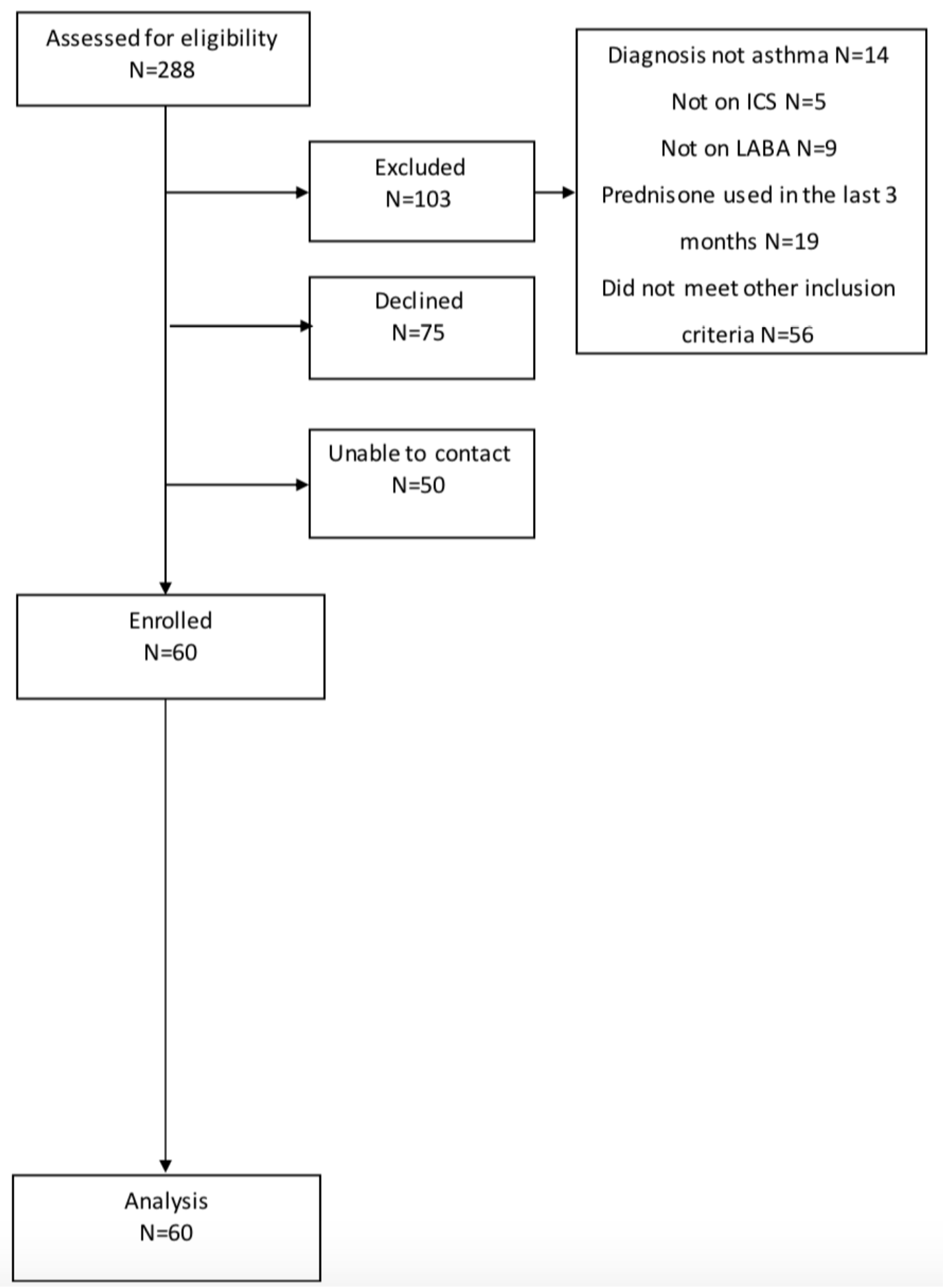


Table 3.4: Participant characteristics for Study Four

\begin{tabular}{|c|c|c|c|}
\hline Continuous variables $(\mathrm{N}=60)$ & Mean (SD) & Median (IQR) & Min to Max \\
\hline Age (years) & $49.8(15.7)$ & 48.8 (37.7 to 63.8) & 21.0 to 75.5 \\
\hline BMI $\left(\mathrm{kg} / \mathrm{m}^{2}\right)$ & $28.9(7.7)$ & 27.8 (24.2 to 32.4 ) & 18.3 to 68.9 \\
\hline $\mathrm{FEV}_{1} \%$ predicted & $101.5(13.8)$ & 100.4 (92.6 to 110.2 ) & 73.0 to 129.7 \\
\hline ACQ-5 & $0.89(0.88)$ & $0.8(0.1$ to 1.2$)$ & 0 to 3.8 \\
\hline AQLQ(S) & $6.1(1.0)$ & 6.5 (5.8 to 6.7$)$ & 2.1 to 7.0 \\
\hline Mean ICS dose (FP & $478(205)$ & 500 (400 to 500$)$ & 100 to 1000 \\
\hline \multicolumn{4}{|l|}{ equivalent/day) } \\
\hline Eosinophil count (x 10\%/L) & $0.27(0.18)$ & $0.20(0.15$ to 0.30$)$ & 0 to 0.8 \\
\hline FeNO (ppb) & $24.6(16.6)$ & 19.0 (13.0 to 29.5$)$ & 5.0 to 75.0 \\
\hline Logarithm FeNO (ppB) & $3.0(0.62)$ & 2.94 (2.56 to 3.38 ) & 1.61 to 4.33 \\
\hline Periostin (ng/ml) & $52.2(16.4)$ & 48.9 (41.6 to 60.3$)$ & 23.2 to 106.6 \\
\hline Logarithm Periostin (ng/ml) & $3.91(0.30)$ & 3.89 (3.73 to 4.10$)$ & 3.15 to 4.67 \\
\hline Categorical Variables & $\mathrm{N} / 60(\%)$ & & \\
\hline Female & $34(56.7)$ & & \\
\hline \multicolumn{4}{|l|}{ Ethnicity: } \\
\hline European & $48(80)$ & & \\
\hline Maori & $5(8.3)$ & & \\
\hline Pacific & $2(3.3)$ & & \\
\hline Asian & $3(5)$ & & \\
\hline Other & $2(3.3)$ & & \\
\hline Current smoker & $4(6.7)$ & & \\
\hline Rhinosinusitis & $46(76.7)$ & & \\
\hline
\end{tabular}

SD: standard deviation, IQR: interquartile range, BMI: body mass index, FEV1: forced expiratory volume in one second, ACQ: asthma control questionnaire, AQLQL(S): standardised asthma quality of life questionnaire, ICS: inhaled corticosteroid, FP: fluticasone propionate, FeNO: Fraction of exhaled nitric oxide, ppb: parts per billion, ng; nanogram, ml: millilitre. 
Periostin values and variability

Individual periostin values per visit are shown in Table 3.5, at the end of this section. The range of periostin values was $23 \cdot 2 \mathrm{ng} / \mathrm{ml}$ to $106 \cdot 6 \mathrm{ng} / \mathrm{ml}$, with a mean (SD) $52 \cdot 2 \mathrm{ng}$ / $\mathrm{ml}(16.4)$ and median $48.9 \mathrm{ng} / \mathrm{ml}$. Individual participant mean periostin levels were ranked and are depicted in Figure 3.5.

Figure 3.5: Individual participant mean periostin plus or minus one standard deviation ranked by mean periostin.

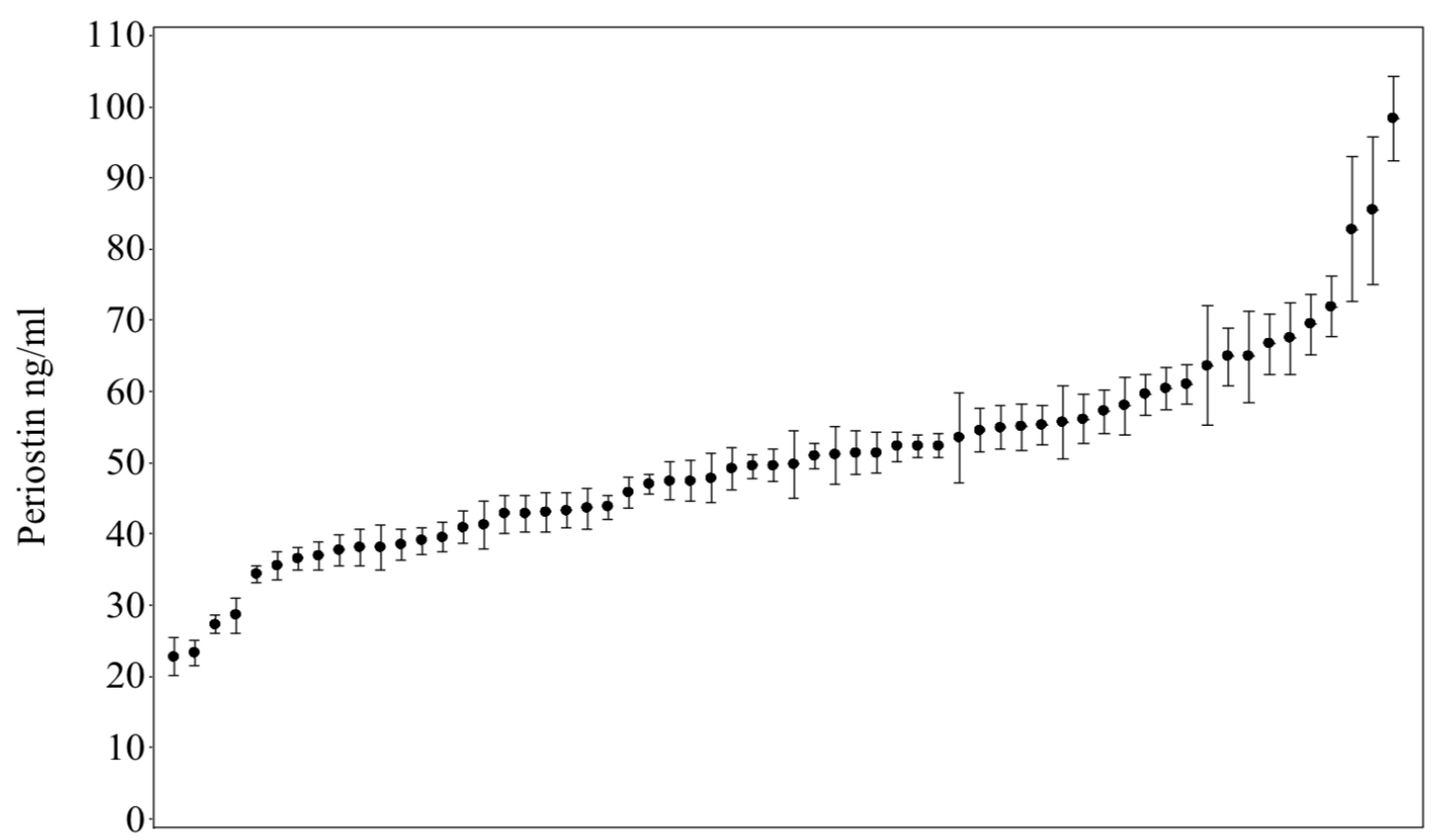

Sequential Bland-Altman plots (Figure 3.6) illustrate examples of between-participant variability of periostin at each visit compared to their baseline value, and the results are summarised in Table 3.6 corresponding to these plots but also for the comparison between consecutive visits (plots not shown). The estimate of inter-participant variance in periostin, based on the raw-scale measurements was $193 \cdot 6$, which was large in comparison to the intra-participant variance of $15 \cdot 3$, with an estimated ICC of 0.93 . This is consistent with low variability of periostin within individuals in a clinical 
sample representing a well-controlled adult asthmatic population. The $95 \% \mathrm{Cl}$ for individual predictions of periostin levels were plus or minus $7 \cdot 7 \mathrm{ng} / \mathrm{ml}$. The mean coefficient of variation $(95 \% \mathrm{Cl})$ for the periostin for the 60 participants was $6.3(5.7$ to $7 \cdot 0) \%$. 
Table 3.6: Limits of agreement for Periostin (raw values) for a particular visit minus baseline and for adjacent visits

\begin{tabular}{|c|c|c|c|c|}
\hline \multirow[b]{2}{*}{ From Baseline Visit } & \multirow[b]{2}{*}{ Bias } & \multirow[b]{2}{*}{ Paired SD } & \multicolumn{2}{|c|}{ Limits of agreement } \\
\hline & & & Lower & Upper \\
\hline 2 & -0.68 & 3.81 & -8.3 & 6.94 \\
\hline 3 & -2 & 4.35 & -10.7 & 6.7 \\
\hline 4 & -1.49 & 4.76 & -11.01 & 8.03 \\
\hline 5 & -1.73 & 5.53 & -12.79 & 9.33 \\
\hline 6 & -1.77 & 6.05 & -13.87 & 10.33 \\
\hline 7 & -2.28 & 6.35 & -14.98 & 10.42 \\
\hline 8 & -2.02 & 6.48 & -14.98 & 10.94 \\
\hline 9 & -1.56 & 5.92 & -13.4 & 10.28 \\
\hline 10 & -2.58 & 5.94 & -14.46 & 9.3 \\
\hline 11 & -2.88 & 5.93 & -14.74 & 8.98 \\
\hline \multicolumn{5}{|c|}{ Adjacent visit comparison } \\
\hline 3 and 2 & -1.4 & 3.23 & -7.86 & 5.06 \\
\hline 4 and 3 & 0.42 & 3.14 & -5.86 & 6.7 \\
\hline 5 and 4 & -0.24 & 3.69 & -7.62 & 7.14 \\
\hline 6 and 5 & 0.04 & 4.49 & -8.94 & 9.02 \\
\hline 7 and 6 & -0.72 & 4.40 & -9.52 & 8.08 \\
\hline 8 and 7 & 0.49 & 5.62 & -10.75 & 11.73 \\
\hline 8 and 9 & 0.46 & 5.08 & -9.7 & 10.62 \\
\hline 10 and 9 & -1.04 & 5.18 & -11.4 & 9.32 \\
\hline 11 and 10 & -0.35 & 4.18 & -8.71 & 8.01 \\
\hline
\end{tabular}

Note: Visit 2 versus 1 is the same as Visit 2 versus baseline 
Figure 3.6: Sequential Bland-Altman plots depicting Limits of Agreement, illustrating between participant variability at consecutive visits compared to baseline.

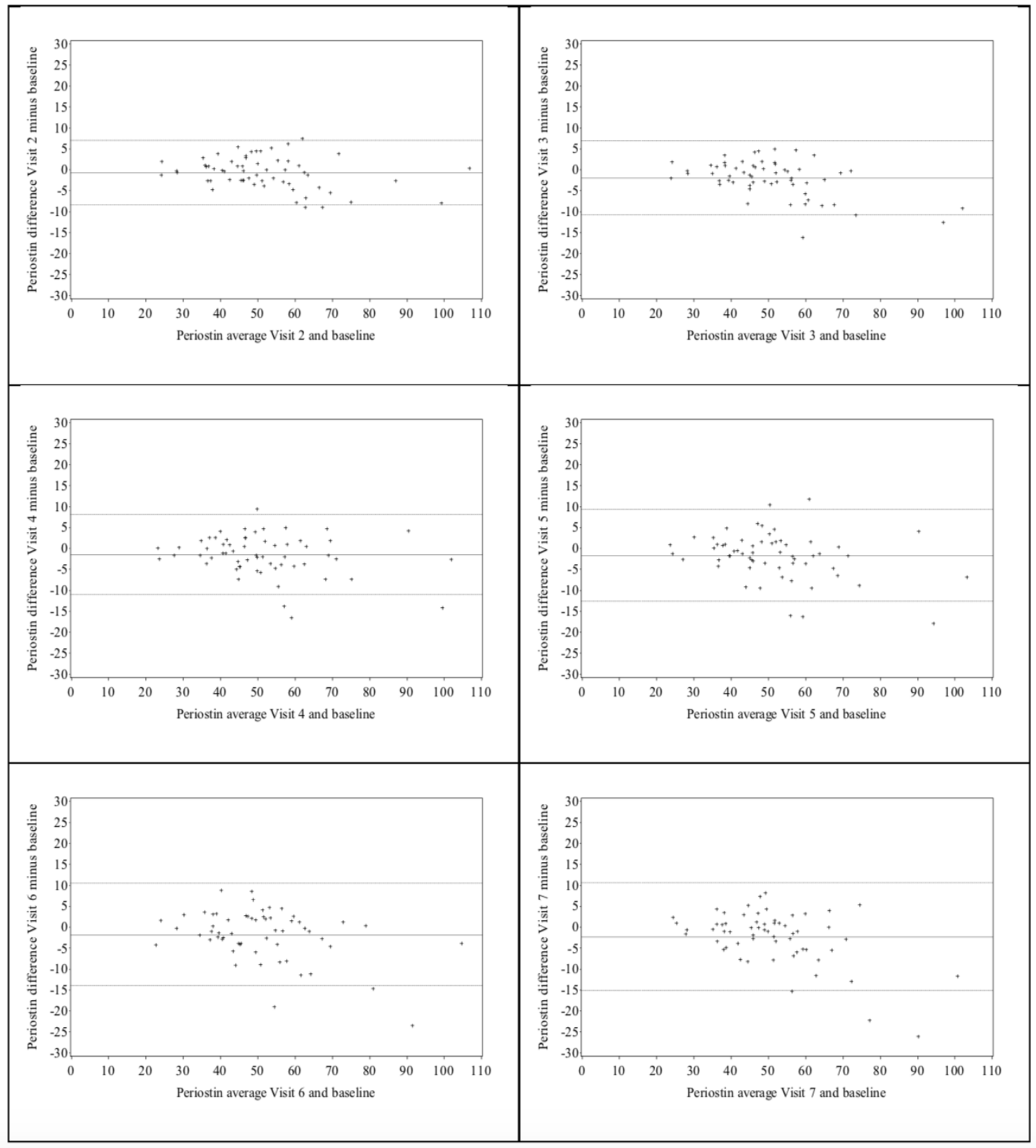




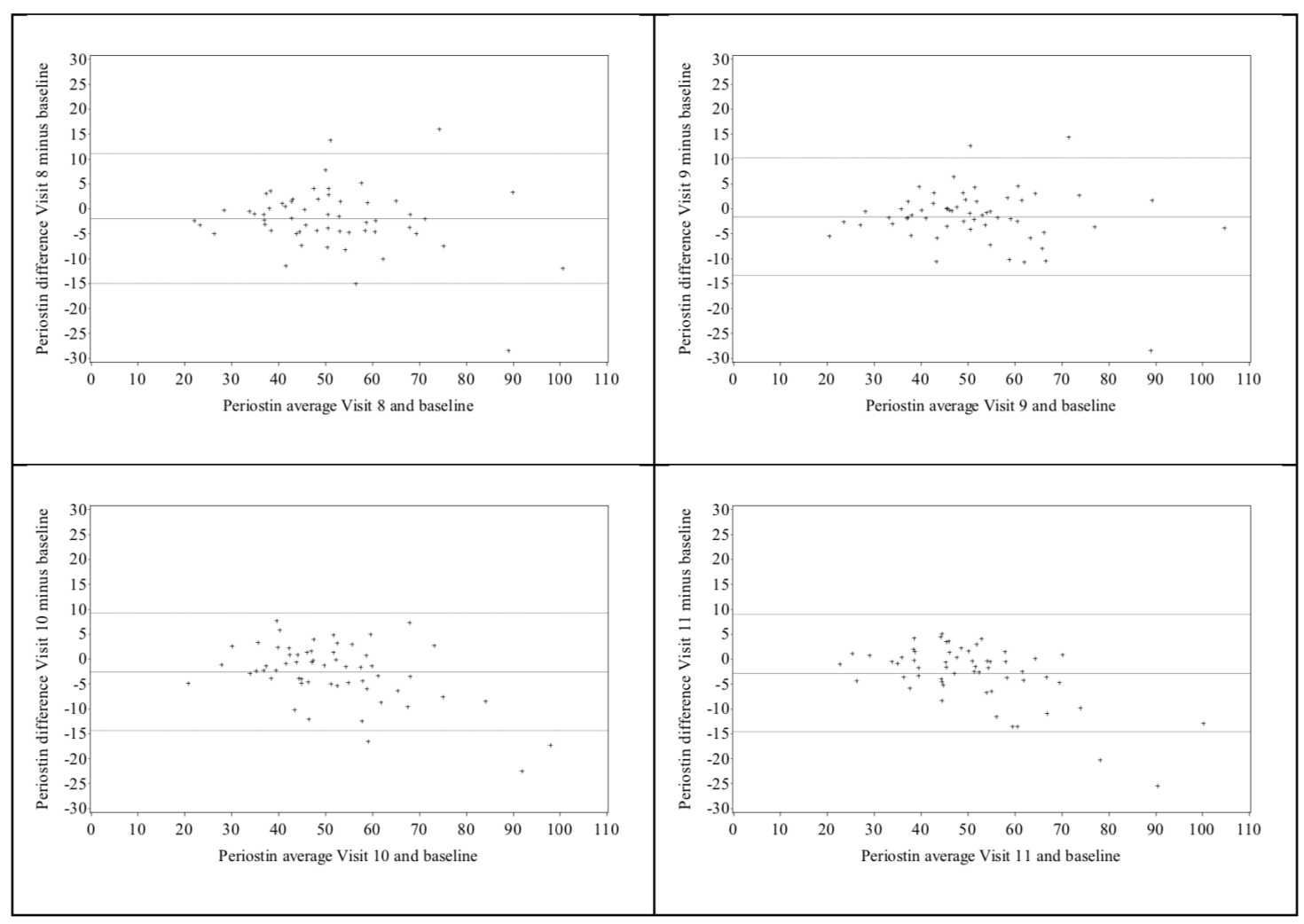

Seasonal and Monthly variability

There was no evidence of monthly, $\mathrm{P}=0 \cdot 56$, or seasonal, $\mathrm{P}=0 \cdot 90$, variation (Figures 3.7 and 3.8). The least squares means of periostin by month and season, which take into account the repeated measurements on the same participants, are shown in Tables 3.7 and 3.8. 
Figure 3.7: Box plot of mean periostin by month, the horizontal lines are the first quartile, the median and third quartiles, the whiskers extend from maximum to the minimum values and the symbol is the mean.

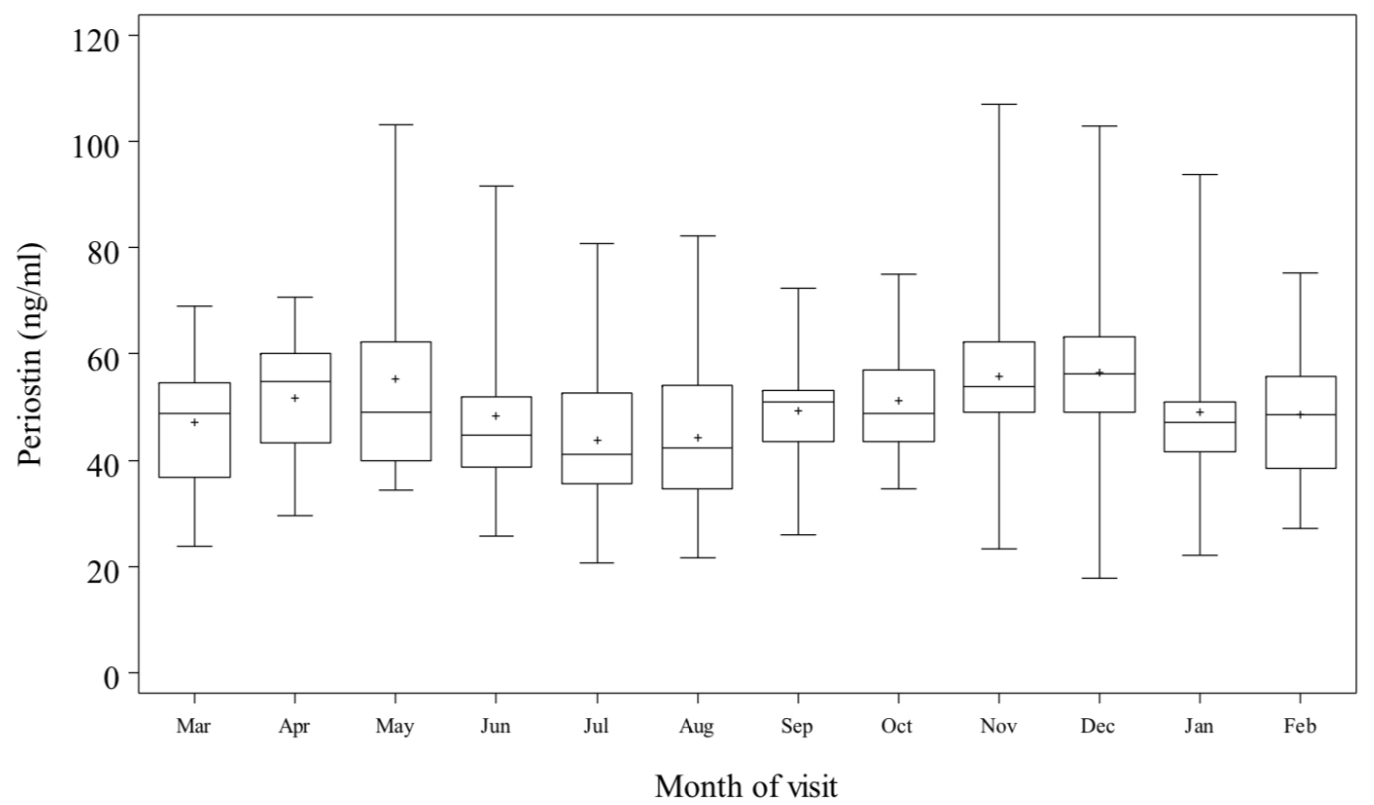

Figure 3.8: Box plot of mean periostin by season, the horizontal lines are the first quartile, the median and third quartiles, the whiskers extend from maximum to the minimum values and the symbol is the mean.

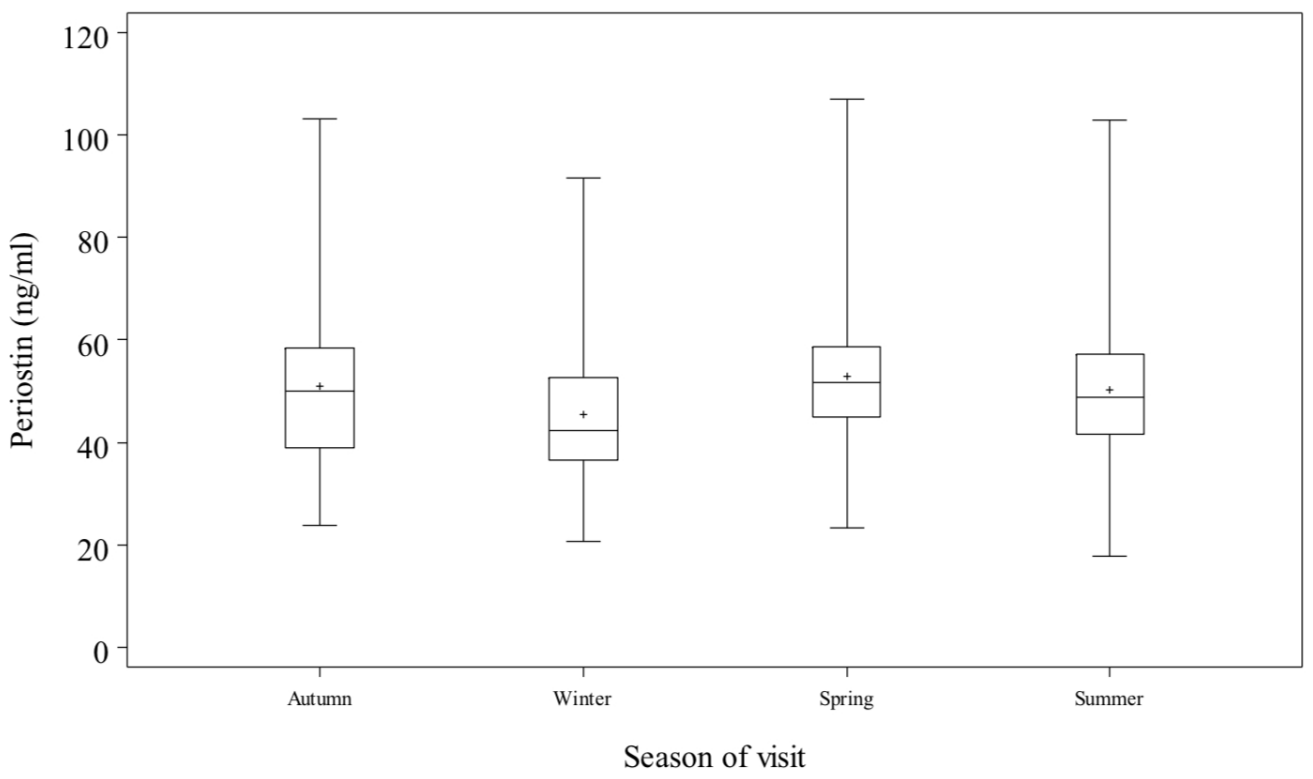


Table 3.7: Least squares mean estimates for Periostin $(\mathrm{ng} / \mathrm{ml})$ by month

\begin{tabular}{|c|c|}
\hline Month & Least squares mean estimate $(95 \% \mathrm{Cl})$ \\
\hline January & 50.1 (46.1 to 54.1$)$ \\
\hline February & 49.6 (45.6 to 53.6$)$ \\
\hline March & 49.3 (45.3 to 53.4$)$ \\
\hline April & 50.5 (46.4 to 54.7$)$ \\
\hline May & 51.3 (46.9 to 55.6$)$ \\
\hline June & 50.8 (46.3 to 55.2$)$ \\
\hline July & 49.8 (45.4 to 54.2 ) \\
\hline August & 49.8 (45.0 to 54.6$)$ \\
\hline September & 49.8 (45.4 to 54.2 ) \\
\hline October & 50.6 (46.5 to 54.7$)$ \\
\hline November & 51.6 (47.5 to 55.6$)$ \\
\hline December & $51.0(46.9$ to 55.1$)$ \\
\hline
\end{tabular}

Table 3.8: Least squares mean estimates for Periostin $(\mathrm{ng} / \mathrm{ml})$ by season

\begin{tabular}{|c|c|}
\hline Season & Least squares mean estimate $(95 \% \mathrm{Cl})$ \\
\hline Autumn & 50.3 (46.6 to 54.1$)$ \\
\hline Winter & 49.8 (45.8 to 53.8 ) \\
\hline Spring & 50.7 (47.0 to 54.5$)$ \\
\hline Summer & 50.3 (46.6 to 54.0$) \mid$ \\
\hline
\end{tabular}


Periostin changes in relation to severe asthma exacerbations

Five participants had a severe asthma exacerbation during the study and required a course of systemic corticosteroids (Figure 3.9) with a mean dose of 35mg/day (range 20-60mg/day, courses lasting from one day to 16 days. The mixed linear model estimate $(95 \% \mathrm{Cl})$ of the difference in periostin between being on systemic corticosteroids and the pre-exacerbation measurements for these five participants was $-5 \cdot 4 \mathrm{ng} / \mathrm{ml}(-8 \cdot 3$ to $-2 \cdot 6), \mathrm{P}=0 \cdot 002$. The difference $(95 \% \mathrm{Cl})$ in periostin between the post- and pre-exacerbation levels was $-4.5 \mathrm{ng} / \mathrm{ml}(-7 \cdot 0$ to $-1 \cdot 9), P<0.001$ which is smaller than the $95 \% \mathrm{Cl}$ for an individual prediction of periostin.

Figure 3.9: Joined line plot by time of the raw periostin levels in the 5 participants that had a severe exacerbation, with prednisone use marked as an event. An arrow marks a periostin measurement taken when prednisone was being used for an exacerbation.

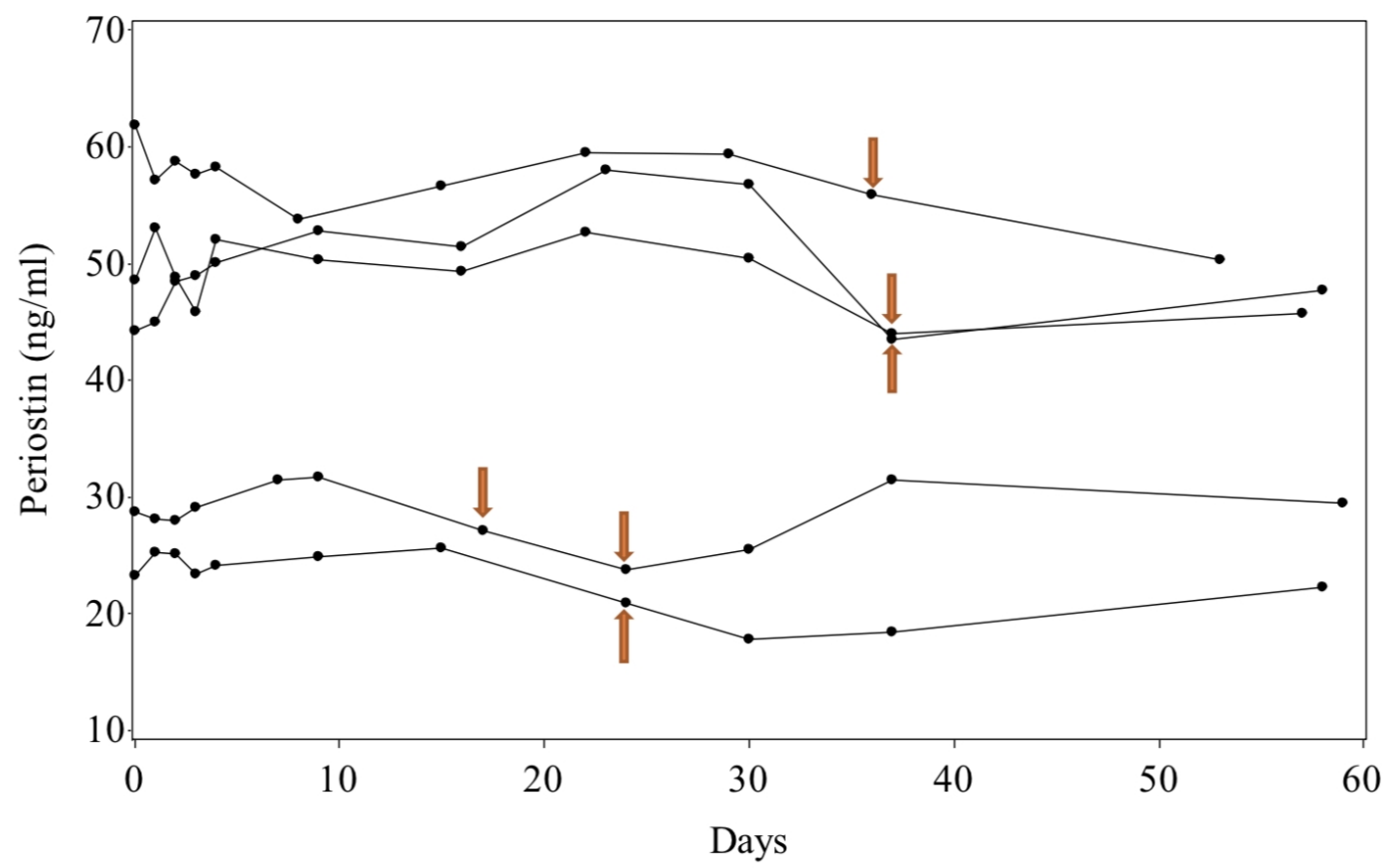


Cut-point analysis

By Day 5, 3/29 participants (10.3\%) changed classification from 'high' periostin levels to 'low' periostin levels and 5/31 (16.1\%) participants changed from 'low' periostin levels to 'high' periostin levels. At Day 24, 4/27 participants (14.8\%) changed classification from 'high' to 'low' and the same proportion changed from 'low' to 'high'.

On Day 59, no participant changed from 'high' to 'low' and 1/30 participant (3.3\%) changed from 'low' to 'high', when compared with Day 1. These changes were not statistically significant (Table 3.9). 
Table 3.9. Cut point analysis of periostin levels $(\mathrm{ng} / \mathrm{ml})$, Days 5,24 and 59 versus baseline. Periostin $>50 \mathrm{ng} / \mathrm{ml}$ is 'High'.

\section{Day 5 periostin $(\mathrm{N}=60)$}

\begin{tabular}{lcccc}
\hline & \multicolumn{5}{c}{ Difference in proportions } \\
\hline Day 1 periostin & High & Low & $\%(95 \% \mathrm{Cl})$ & P Value \\
\hline High & 26 & 3 & $3.3(-2.9$ to 12.5$)$ & 0.48 \\
Low & 5 & 26 & & \\
\hline
\end{tabular}

Day 28 periostin $(\mathrm{N}=57)$

\begin{tabular}{|c|c|c|c|c|}
\hline \multirow[b]{2}{*}{ Day 1 periostin } & \multicolumn{4}{|c|}{ Difference in proportions } \\
\hline & High & Low & $\%(95 \% \mathrm{Cl})$ & P Value \\
\hline \multirow[t]{2}{*}{ High } & 23 & 4 & \multirow{3}{*}{$0(-9.7$ to 9.7$)$} & \multirow{3}{*}{0.99} \\
\hline & & & & \\
\hline \multirow[t]{3}{*}{ Low } & 4 & 26 & & \\
\hline & Day 5 & eriosti & 58) & \\
\hline & \multicolumn{4}{|c|}{ Difference in proportions } \\
\hline Day 1 periostin & High & Low & $\%(95 \% \mathrm{Cl})$ & P Value \\
\hline \multirow[t]{2}{*}{ High } & 28 & 0 & \multirow{3}{*}{$1.7(-1.6$ to 5.7$)$} & \multirow{3}{*}{0.31} \\
\hline & & & & \\
\hline Low & 1 & 29 & & \\
\hline
\end{tabular}


Table 3.5: Periostin measurement by participant and day (Visit Day 1 is the baseline measurement

\begin{tabular}{|c|c|c|c|c|c|c|c|c|c|c|c|}
\hline & Serum & eriostin & $(\mathrm{ng} / \mathrm{ml})$ & & & & & & & & \\
\hline & 1 & 2 & 3 & 4 & 5 & 10 & 17 & 24 & 31 & 38 & 59 \\
\hline 1 & 35.77 & 36.54 & 36.51 & 38.32 & 36.72 & 44.64 & 36.55 & 38.87 & 35.81 & 43.53 & 36.16 \\
\hline 2 & 60.57 & 61.56 & 64.04 & 62.41 & 62.14 & 61.81 & 54.64 & 56.26 & 62.25 & 59.20 & NA \\
\hline 3 & 43.70 & 41.40 & 43.04 & 43.14 & 42.45 & 42.24 & 39.89 & 41.8 & 50.2 & 44.52 & 47.18 \\
\hline 4 & 58.30 & 55.39 & 54.84 & 54.39 & 54.77 & 60.88 & 57.35 & 50.09 & 51.06 & 56.63 & 51.82 \\
\hline 5 & 52.54 & 49.90 & 49.15 & 47.16 & 43.07 & 46.53 & 50.26 & 48.63 & 48.47 & 40.46 & 50.10 \\
\hline 6 & 37.37 & 41.26 & 39.14 & 40.01 & 38.12 & 40.55 & 37.94 & 36.22 & 41.86 & 43.18 & 39.37 \\
\hline 7 & 88.31 & 85.67 & NA & 92.51 & 92.43 & 73.62 & 66.05 & 91.63 & 90.11 & 79.87 & 68.07 \\
\hline 8 & 38.66 & 36.04 & 35.13 & 36.48 & 34.48 & 35.65 & 37.68 & 35.54 & 37.40 & 41.06 & 38.46 \\
\hline 9 & 103.20 & 95.24 & 90.69 & 100.60 & 85.36 & 79.77 & 77.09 & 74.82 & 74.78 & 80.71 & 77.73 \\
\hline 10 & 46.31 & 46.05 & 44.64 & 43.17 & 45.30 & 40.55 & 38.58 & 41.33 & 40.53 & 42.43 & 42.36 \\
\hline 11 & 28.42 & 28.12 & 28.14 & 26.84 & 25.83 & 28.14 & 27.78 & 28.23 & 27.92 & 27.33 & 24.08 \\
\hline 12 & 48.56 & 52.99 & 48.77 & 45.86 & 52.00 & 50.34 & 49.32 & 52.72 & 50.45 & 43.94 & 45.66 \\
\hline 13 & 40.59 & 40.42 & 38.13 & 42.72 & 38.84 & 38.17 & 35.30 & 36.27 & 35.23 & 38.39 & 34.70 \\
\hline 14 & 40.30 & 35.57 & 38.81 & 41.27 & 38.61 & 38.88 & 39.24 & 41.38 & 40.01 & 36.42 & 38.57 \\
\hline 15 & 49.26 & 50.80 & 54.27 & 54.01 & 53.85 & 53.40 & 48.62 & 52.12 & 53.57 & 54.09 & 50.87 \\
\hline 16 & 24.87 & 23.59 & 22.87 & 22.40 & 23.61 & 20.68 & 25.89 & 21.62 & 22.21 & NA & 25.96 \\
\hline 17 & 66.24 & 59.52 & 63.84 & 70.96 & 56.84 & NA & 66.25 & 82.27 & 60.40 & 57.58 & 52.67 \\
\hline 18 & 51.12 & 56.33 & 52.49 & 52.92 & 52.70 & 53.13 & 52.15 & 50.03 & 52.64 & 52.43 & 50.69 \\
\hline 19 & 41.97 & 47.49 & 43.95 & 42.88 & 43.92 & 39.54 & 44.97 & 43.51 & 40.13 & 41.15 & 46.48 \\
\hline
\end{tabular}




\begin{tabular}{|c|c|c|c|c|c|c|c|c|c|c|c|}
\hline 20 & 37.90 & 35.33 & 38.84 & 42.00 & 38.91 & 38.14 & 34.61 & 38.01 & 36.00 & 35.62 & 39.38 \\
\hline 21 & 60.10 & 56.78 & 51.79 & 51.01 & 52.38 & 51.73 & 53.21 & 57.42 & 58.11 & 55.79 & 56.38 \\
\hline 22 & 72.26 & 66.81 & 71.97 & 69.82 & 70.51 & 73.49 & 69.39 & 70.25 & 75.05 & 62.68 & 61.36 \\
\hline 23 & 36.44 & 37.29 & 39.98 & 36.5 & 41.27 & 39.53 & 39.95 & 40.06 & 37.99 & 34.04 & 40.64 \\
\hline 24 & 54.26 & 56.47 & 54.32 & 54.99 & 55.16 & 58.72 & 54.61 & 46.59 & 53.53 & 57.2 & 53.83 \\
\hline 25 & 41.98 & 44.00 & 39.01 & 40.92 & 41.45 & 39.08 & 47.14 & 44.02 & 43.16 & 42.83 & 47.07 \\
\hline 26 & 64.29 & 56.48 & 57.10 & 60.62 & 63.02 & 63.36 & 68.29 & 65.87 & 78.76 & 71.57 & 64.46 \\
\hline 27 & 46.15 & 50.51 & 46.72 & 46.62 & 43.93 & 48.70 & 47.45 & 53.97 & 45.86 & 47.71 & 44.55 \\
\hline 28 & 67.32 & 58.34 & 51.17 & 50.75 & 51.01 & 55.88 & 59.48 & 57.29 & 56.67 & 50.81 & 53.75 \\
\hline 29 & 47.40 & 44.91 & 44.38 & 43.08 & 44.44 & 43.61 & 47.23 & 44.19 & 47.76 & 47.21 & 49.65 \\
\hline 30 & 68.57 & 64.3 & 60.05 & 70.38 & 69.00 & 65.82 & 57.00 & 67.43 & 63.87 & 62.26 & 64.94 \\
\hline 28 & 67.32 & 58.34 & 51.17 & 50.75 & 51.01 & 55.88 & 59.48 & 57.29 & 56.67 & 50.81 & 53.75 \\
\hline 29 & 47.40 & 44.91 & 44.38 & 43.08 & 44.44 & 43.61 & 47.23 & 44.19 & 47.76 & 47.21 & 49.65 \\
\hline 30 & 68.57 & 64.30 & 60.05 & 70.38 & 69.00 & 65.82 & 57.00 & 67.43 & 63.87 & 62.26 & 64.94 \\
\hline 31 & 52.34 & 52.38 & 51.56 & 50.30 & 54.24 & 54.63 & 53.37 & 53.85 & 50.24 & 52.20 & 50.79 \\
\hline 32 & 45.41 & 48.23 & 46.37 & 47.97 & 46.07 & 48.19 & 45.25 & 49.55 & 45.50 & 46.79 & 46.75 \\
\hline 33 & 53.63 & 49.75 & 50.77 & 47.89 & 52.72 & 51.08 & 50.24 & 52.09 & 52.44 & 48.62 & 51.07 \\
\hline 34 & 57.17 & 59.24 & 55.23 & 52.44 & 50.33 & 56.26 & 54.39 & NA & 55.44 & 62.14 & 58.68 \\
\hline 35 & 47.37 & 51.80 & 49.31 & 51.35 & 49.03 & 49.48 & 51.70 & 49.35 & 50.56 & 46.73 & 47.78 \\
\hline 36 & 61.86 & 57.17 & 58.75 & 57.66 & 58.20 & 53.73 & 56.62 & 59.50 & 59.32 & 55.84 & 50.34 \\
\hline 37 & 71.82 & 62.91 & 63.56 & 64.54 & 65.41 & 67.17 & 77.13 & 66.82 & 61.34 & 74.61 & 67.14 \\
\hline 38 & 62.82 & 62.18 & 57.06 & 63.37 & 61.03 & 62.60 & 57.50 & 58.26 & 65.88 & 59.52 & 60.35 \\
\hline
\end{tabular}




\begin{tabular}{|c|c|c|c|c|c|c|c|c|c|c|c|}
\hline 39 & 106.60 & 107.00 & 97.42 & 92.45 & 99.74 & 102.70 & 94.92 & 94.70 & 102.8 & 89.31 & 93.69 \\
\hline 40 & 23.23 & 25.27 & 25.06 & 23.40 & 24.08 & 24.83 & 25.58 & 20.82 & 17.77 & 18.38 & 22.19 \\
\hline 41 & 78.81 & 71.17 & 68.01 & 71.51 & 69.99 & 79.17 & 65.84 & 71.42 & 75.22 & 71.29 & 69.05 \\
\hline 42 & 33.99 & 36.85 & 35.16 & 35.83 & 36.59 & 37.59 & 38.33 & 33.48 & 32.28 & 37.32 & 33.52 \\
\hline 43 & 46.81 & 44.33 & 43.14 & 41.82 & 44.14 & 42.91 & 44.89 & 42.20 & 46.39 & 42.80 & 42.19 \\
\hline 44 & 50.35 & 49.08 & 47.64 & 48.77 & 51.57 & 52.80 & 49.42 & 45.96 & 47.90 & 49.05 & 53.28 \\
\hline 45 & 48.58 & 46.58 & 40.47 & 41.26 & 39.36 & 39.46 & 40.38 & 41.24 & 38.01 & 38.34 & 40.23 \\
\hline 46 & 41.15 & 40.84 & 41.55 & 40.07 & 40.47 & 42.93 & 36.29 & 41.68 & 44.44 & 43.35 & 37.86 \\
\hline 47 & 45.54 & 46.39 & 44.34 & 47.98 & 51.04 & 52.16 & 48.86 & 45.44 & 45.60 & 49.46 & 44.98 \\
\hline 48 & 47.31 & 44.82 & 42.68 & 42.97 & 42.76 & 43.20 & 44.55 & 35.90 & 43.78 & 42.49 & 42.08 \\
\hline 49 & 50.85 & 47.40 & 52.60 & 48.80 & 47.33 & 55.58 & 52.46 & NA & 50.02 & 54.11 & 54.98 \\
\hline 50 & 38.12 & 38.40 & 35.48 & 34.51 & 35.35 & 37.08 & 39.02 & 35.91 & 36.33 & 36.70 & 34.57 \\
\hline 51 & 28.73 & 28.10 & 27.92 & 29.04 & 31.47 & 31.73 & 27.12 & 23.76 & 25.51 & 31.40 & 29.46 \\
\hline 52 & 44.17 & 45.02 & 48.44 & 48.93 & 50.09 & 52.76 & 51.45 & 58.01 & 56.81 & 43.54 & 47.76 \\
\hline 53 & 58.29 & 65.74 & 58.40 & 56.27 & 55.78 & 59.76 & 61.55 & 59.52 & 62.94 & 59.01 & 57.76 \\
\hline 54 & 35.36 & 36.51 & 34.55 & 33.77 & 35.48 & 33.55 & 34.91 & 34.34 & 32.40 & 32.49 & 34.55 \\
\hline 55 & 55.25 & 53.31 & 54.91 & 51.59 & 50.60 & 46.37 & 47.41 & 50.74 & 52.02 & 49.86 & 53.49 \\
\hline 56 & 55.03 & 61.25 & 59.71 & 59.95 & 66.78 & 54.33 & 57.83 & 60.20 & 54.55 & 53.55 & 54.54 \\
\hline 57 & 69.76 & 73.66 & 68.96 & 68.12 & 64.99 & 58.64 & 64.33 & 66.08 & 61.79 & 66.34 & 70.57 \\
\hline 58 & 45.14 & 48.45 & 49.64 & 54.52 & 55.59 & NA & 53.28 & NA & NA & NA & NA \\
\hline 59 & 57.32 & 57.33 & 54.87 & 58.36 & 55.52 & 53.17 & 55.78 & 52.59 & 59.50 & 52.54 & 50.64 \\
\hline 60 & 64.01 & 62.74 & 55.86 & 50.18 & 47.94 & 45.03 & 48.74 & 48.98 & 53.77 & 51.59 & 59.75 \\
\hline
\end{tabular}




\subsubsection{Discussion}

This longitudinal study investigating periostin variability in stable adult asthmatics, treated with ICS and LABA therapy, found little variability in periostin measurements within-individuals over a 59 day period. The within-participant variance was $15 \cdot 3$ with the $95 \%$ confidence interval for an individual measured repeatedly of plus or minus $7 \cdot 7 \mathrm{ng} / \mathrm{ml}$. Variance between participants was much larger than the within participant variation in this relatively homogenous asthmatic population.

These findings are consistent with studies performed in populations with severe asthma ${ }^{53,98}$ over a period of 5 and 2 weeks respectively. These studies reported a coefficient of variation, rather than variance components, with a point estimate of $5.3 \%$ and $5 \cdot 0 \%$, respectively, compared to our estimate of $6 \cdot 3 \%$. This is consistent with the low intra-participant variability in periostin measurements observed in this study.

The distribution of serum periostin levels in our study sample was relatively wide, ranging from 23.2 to $106.6 \mathrm{ng} / \mathrm{ml}$, with a median of $48 \cdot 9 \mathrm{ng} / \mathrm{ml}$ and mean $52 \cdot 2 \mathrm{ng} / \mathrm{ml}$. These levels are similar to those found in a sample representative of a population without respiratory disease (range $28 \cdot 1$ to $136 \cdot 4 \mathrm{ng} / \mathrm{ml}$, median $50 \cdot 1 \mathrm{ng} / \mathrm{ml}$ ) ${ }^{174}$ and, in a sample of adults with symptomatic obstructive airways disease (range $15 \cdot 0$ to $164 \cdot 7 \mathrm{ng}$ / $\mathrm{ml}$, median $54 \cdot 0 \mathrm{ng} / \mathrm{ml})^{100}$ and those reported in a population of severe asthmatics who

received monoclonal antibody therapy against IgE (median 50ng/ml) ${ }^{69}$ and $\mathrm{IL}-13$ (median $50.2 \mathrm{ng} / \mathrm{ml}$ ). ${ }^{53}$ Together, these findings suggest that serum periostin levels do not differentiate patients with asthma from individuals without asthma and nor does it 
distinguish between different levels of asthma severity for those that have asthma. However, within the asthma population, it has been reported to identify patients who might benefit from targeted therapies to IL-13, ${ }^{53,54} \mathrm{IL}-4 \mathrm{R} \alpha^{52}$ and IgE. ${ }^{69}$

There was no evidence of a monthly or seasonal fluctuation in serum periostin in this population who were recruited over a 12 month period. The lack of seasonal variation in periostin levels is consistent with the findings of Kanemitsu and colleagues who found no seasonal variation in a sample of 224 asthmatic patients who averaged $16 \cdot 2$ measurements of periostin over 8 years. ${ }^{97}$ Thus it is not necessary to consider seasonality when assessing periostin levels in candidates for treatment with asthma biologic therapies.

In the five participants who suffered a severe exacerbation of asthma during the study, there was a reduction in periostin of $4.5 \mathrm{ng} / \mathrm{ml}$ after the exacerbation and after treatment with a course of systemic corticosteroid. This reduction is smaller than the $95 \% \mathrm{Cl}$ for an individual prediction in those with clinically stable asthma. It has previously been shown that periostin levels fall by a similar amount $(-4 \cdot 8 \mathrm{ng} / \mathrm{ml})$ in hitherto steroid naïve patients with symptomatic obstructive airways disease after the initiation of 12 weeks of ICS therapy. ${ }^{100}$ Together, these findings indicate the serum periostin levels are reduced by corticosteroids, although the modest $10 \%$ decrease after administration of both inhaled and systemic corticosteroids suggests that periostin release is primarily a consequence of physiological processes rather than Type 2 inflammation. This contrasts with FeNO, in which inhaled or systemic corticosteroid treatment result in around a 20\% reduction in FeNO levels. ${ }^{201}$ 
The periostin cut-point of $\geq 50 \mathrm{ng} / \mathrm{ml}$ was originally proposed to identify patients with moderately severe asthma, on ICS and LABA therapy, who could potentially derive enhanced benefit from monoclonal antibody treatment directed against IL-13. ${ }^{10}$ This cut-point was also reported as a predictor of responsiveness to monoclonal antibody therapy directed against $\mathrm{IL}-13^{10}$ and IgE. ${ }^{11}$ Using this cut-point, $10 \%$ and $16 \%$ of participants classified as 'high' at Day 1 had changed to 'low' on Days 5 and 28 respectively, while $16 \%$ and $16 \%$ classified as 'low' at Day 1, changed to 'high' at Days 5 and 28. This proportion of changing between categories of 'high' and 'low', indicates differentiated responsiveness to monoclonal antibody therapy, is perhaps not surprising given the median periostin level in this sample was $48.9 \mathrm{ng} / \mathrm{ml}$.

There are some methodological issues relevant to the interpretation of the study findings. The participants in the study were all taking regular ICS and LABA therapy in an attempt to investigate a population in whom monoclonal antibody therapy may be used. However the subjects had well controlled asthma and as a result, the findings of this study may not be generalisable to those with unstable asthma or severe airflow obstruction. Nor would the findings be generalisable to smokers, in whom the periostin levels have shown to be lower. ${ }^{174}$ In a more general clinical population where there are additional causes of variation in periostin, such as recent surgical or dental procedures, there may be greater within-patient variability in periostin levels. Additionally the participants in this study were primarily New Zealand European (80\%) and thus our results are generalisable to Caucasian populations. 
In conclusion, the $95 \%$ individual prediction for periostin in stable asthmatics on ICS and LABA over a 59 day period is plus or minus $7 \cdot 7 \mathrm{ng} / \mathrm{ml}$. There was no evidence of seasonal fluctuation in periostin levels in this sample. Periostin levels are modestly reduced by systemic corticosteroid treatment of a severe asthma exacerbation, but the effect lies within the $95 \%$ confidence interval of serum periostin variability within an individual with stable asthma. These findings suggest that the day-to-day stability of periostin measurements supports its use as a biomarker in asthma, predicting responsiveness to biologic therapy directed against Type 2 disease. 


\section{Chapter 4: Severe exacerbations and Type}

\section{2 biomarkers in asthma}

Biological therapies for asthma target specific pro-inflammatory pathways associated with Type 2 inflammation, characterised by eosinophilic infiltration of the airways. ${ }^{52-}$ 54,69,91 However, identifying potential responders is problematic due to logistical problems obtaining direct evidence of eosinophilic airway inflammation via bronchoscopy or sputum induction, which are time consuming, labour intensive, require specialist facilities and expose the patient to risk. An alternative is to use noninvasive biomarkers, which could both identify patients likely to respond to monoclonal antibody therapy as well as monitor disease activity once on treatment.

Peripheral blood eosinophils, FeNO, serum periostin and serum IgE are all markers of Type 2 inflammation in asthma. Peripheral blood eosinophils, FeNO and serum periostin are predictors of response to treatment with monoclonal antibody therapy directed against $\operatorname{lgE},{ }^{69} \mathrm{IL} 4-\mathrm{Ra},{ }^{52} \mathrm{IL}-5^{202}$ and $\mathrm{IL}-13,,^{53,54}$ whilst monoclonal antibody therapy directed against IgE is recommended in patients within a target serum IgE range. ${ }^{68,203}$ Knowledge of the time course changes of these biomarkers during and after a severe exacerbation of asthma would have clinical utility in identifying requirement and/or eligibility for monoclonal antibody therapies directed against IgE, IL-4Ra, IL-5 and IL-13. 
Severe exacerbations of asthma are a significant public health burden, leading to over 400,000 hospitalisations per year in the United States, costing about half the total of $\$ 11.5$ billion spent on asthma-related healthcare. ${ }^{204}$ Severe asthma exacerbations are associated with accelerated progression of disease, increased morbidity and risk of mortality. ${ }^{205}$ Predictors of severe exacerbations have clinical utility in identifying patients at risk of these outcomes. Clinical features that have been associated with an increased risk of severe exacerbation include poor symptom control, more than one severe exacerbation in the previous year, exposure to tobacco smoke, poor lung function, obesity, major psychosocial problems and high allergen exposures. ${ }^{206}$

Other potential predictors of exacerbations in patients with severe asthma are Type 2 biomarkers, including peripheral blood eosinophils, FeNO, serum periostin and serum IgE. Randomised controlled trials of monoclonal antibody therapies directed against Type 2 asthma have reported that higher levels of blood eosinophils, ${ }^{207} \mathrm{FeNO}^{69}$ and serum periostin ${ }^{52,69}$ are associated with higher exacerbation rates in populations with severe eosinophilic asthma. Serum IgE is another Type 2 biomarker which is associated with an increased risk of exacerbations in asthmatic children. ${ }^{208}$ While these Type 2 biomarkers predict the likelihood of future exacerbations in adults with severe, uncontrolled Type 2 asthma, it is unclear if these associations would extend to a general population of adult asthmatics, across a range of severity. 
From reviewing the literature, it was clear that there was insufficient knowledge regarding the potential for some of these biomarkers to predict future exacerbations. I therefore wished to answer the following questions with Studies Five and Six:

- What effect, if any, does a severe exacerbation and its treatment have on the four Type 2 biomarkers?

- Can these biomarkers potentially predict the risk of future exacerbation of asthma? 


\subsection{Study Five: Change in biomarkers of Type 2 inflammation following severe exacerbations of asthma}

\subsubsection{Introduction}

Blood eosinophils, FeNO, serum periostin and serum IgE are biomarkers of Type 2 inflammation in asthma. Knowledge of their time course during and after severe exacerbations would have clinical utility in identifying eligibility for monoclonal antibody therapy directed against IgE, IL-4Ra, IL-5 and IL-13.

\subsubsection{Hypothesis}

The hypothesis was that the time course of changes in response to the exacerbation and/or its treatment may differ between the biomarkers.

\subsubsection{Objectives}

To describe the change of blood eosinophils, FeNO, serum periostin and serum IgE over 12 weeks after a severe exacerbation of asthma. 


\subsubsection{Methods}

This cohort study recruited 34 adults aged between 18 and 75 years, from Wellington Hospital Emergency Department and participating General Practitioners in the Greater Wellington Region. Participants presented within 24 hours of commencing systemic corticosteroid therapy for a severe exacerbation of asthma. A severe exacerbation was identified in accordance with the American Thoracic Society (ATS) and European Respiratory Society (ERS) criteria ${ }^{209}$, requiring the use of systemic corticosteroids (tablets, suspension or injection), or an increase from a stable maintenance dose, for at least three days or a hospitalisation or Emergency Department visit because of asthma requiring systemic corticosteroids. Courses of corticosteroids separated by more than a week were treated as separate severe exacerbations. Participants were excluded if they had a severe exacerbation in the three months before recruitment or had started systemic corticosteroids more than 24 hours prior to screening. Other exclusion criteria included pregnancy, hospital admission (for a condition other than asthma), surgery requiring general anaesthetic, bone fracture, or dental surgery occurring within three months of enrolment.

Ethical approval was given by the New Zealand Health and Disability Ethics Committee (13/NTB/191). The trial was prospectively registered with the Australia New Zealand Trial Registry (ACTRN12614000443695). Written informed consent was obtained from all participants prior to any study procedures being undertaken. 
Study Procedures

At enrolment, participants completed written informed consent; a General Health Questionnaire; Asthma Control Questionnaire-5 (ACQ-5) and Asthma Quality of Life Questionnaire with Standardised Activities (AQLQ-S); measurement of BMI, $\mathrm{FEV}_{1}$ and FVC, FeNO and blood samples for measurement of full blood count (FBC), creatinine, urea and electrolytes, serum IgE and serum periostin. Further visits were scheduled one, two, four, eight and 12 weeks after commencing systemic corticosteroids, with repeat measurements undertaken as outlined in the study plan (Table 4.1). 
Table 4.1: Study Plan for Study Five

\begin{tabular}{|c|c|c|c|c|c|c|}
\hline Visit No. & 1 & 2 & 3 & 4 & 5 & 6 \\
\hline \multirow[t]{2}{*}{ Time Point } & Day 1 & Week 1 & Week 2 & Week 4 & Week 8 & Week 12 \\
\hline & & \pm 3 days & \pm 3 days & \pm 3 days & \pm 3 days & \pm 3 days \\
\hline Written informed consent & $x$ & & & & & \\
\hline Enrolment & $x$ & & & & & \\
\hline Measurement of BMI & $x$ & & & & & \\
\hline \multicolumn{7}{|l|}{ General Health } \\
\hline & $x$ & & & & & \\
\hline \multicolumn{7}{|l|}{ Questionnaire } \\
\hline ACQ-5 & $x$ & & & & & $x$ \\
\hline AQLQ & $x$ & & & & & $x$ \\
\hline Periostin sample & $x$ & $x$ & $x$ & $x$ & $x$ & $x$ \\
\hline FBC sample & $x$ & $x$ & $x$ & $x$ & $\mathrm{X}$ & $x$ \\
\hline IgE sample & $x$ & $x$ & $x$ & $x$ & $x$ & $x$ \\
\hline FeNO & $x$ & $x$ & $x$ & $x$ & $x$ & $x$ \\
\hline Spirometry (FVC + FEV 1 ) & $x$ & $x$ & $x$ & $x$ & $x$ & $x$ \\
\hline
\end{tabular}


Lung function and FeNO

Spirometry was performed with measurement of $\mathrm{FEV}_{1}$ and FVC using a Masterscreen Pneumo (Masterscreen Version 2·0, Carefusion, Hoechberg, Germany) in accordance with ATS guidelines ${ }^{184}$. FeNO was measured with an online nitric oxide monitor (NiOX, Aerocrine AB, Solna, Sweden) as per ATS guidelines ${ }^{89}$.

Blood samples

The FBC with white cell differential (Sysmex platform, Mundelein, USA), creatinine, urea and electrolytes and serum IgE (Roche, Cobas 501, NZ) were performed immediately in a local laboratory. For serum periostin, blood samples were coagulated, centrifuged and serum aliquots stored at $-80^{\circ} \mathrm{C}$, prior to analysis. Serum periostin levels were measured using the clinical trial version of the Elecsys ${ }^{\circledast}$ Periostin immunoassay (Roche Diagnositics, Penzberg, Germany) described previously ${ }^{115}$.

\section{Study Power}

The sample size of 34 participants had $80 \%$ power, with a type 1 error rate of $5 \%$, to detect a paired difference in periostin of 0.5 standard deviations. This arbitrary difference was used as the clinically meaningful change in serum periostin was uncertain. The study was not powered to detect a pre-specified paired difference in the other Type 2 biomarkers. 


\section{Statistical Methods}

For the purposes of analysis and data description, severe exacerbation onset was marked as the day of commencement of systemic corticosteroids and denoted as 'week zero'. Time was based on the number of weeks after the most recent severe exacerbation so that participants with multiple severe exacerbations have multiple week zero data-points and shorter subsequent follow-up periods than those with only one severe exacerbation.

FeNO, serum periostin and serum IgE have strongly skewed distributions and normality assumptions, for analyses for these variables, were better met on a logarithm transformed scale. The exponent of a difference in logarithms can be interpreted as a ratio of geometric means. Mixed linear models were used to compare the reference 12 week biomarker measurement with those at each previous visit, under the assumption that biomarker levels would return to stable levels by the end of the study, providing there were no further severe exacerbations. A type I error rate of 0.05 and associated 95\% confidence intervals are shown, for individual statistical tests.

SAS $9 \cdot 4$ was used. 


\subsubsection{Results}

The flow of participants is shown in Figure 4.1. A total of 34 participants enrolled in the study as described in Table 4.2. At enrolment, participants' mean $\mathrm{FEV}_{1}$ was $80 \%$ predicted and the mean ACQ-5 score was 3.7. Participants were taking an average of $450 \mu \mathrm{g}$ of fluticasone propionate-equivalent per day with $26 / 34(76 \%)$ on a concurrent long-acting beta agonist (LABA). The average length of systemic corticosteroid prescription was 10.9 days (range one to 28 days) with participants taking an average dose of $36 \mathrm{mg}$ of prednisone per day (range 20 to $60 \mathrm{mg}$ per day). Most participants had a single severe exacerbation over the 12 weeks (25/34, 74\%), with six, two and one participant having a two, three and four severe exacerbations, respectively. 
Figure 4.1: Flow of participants through Study Five

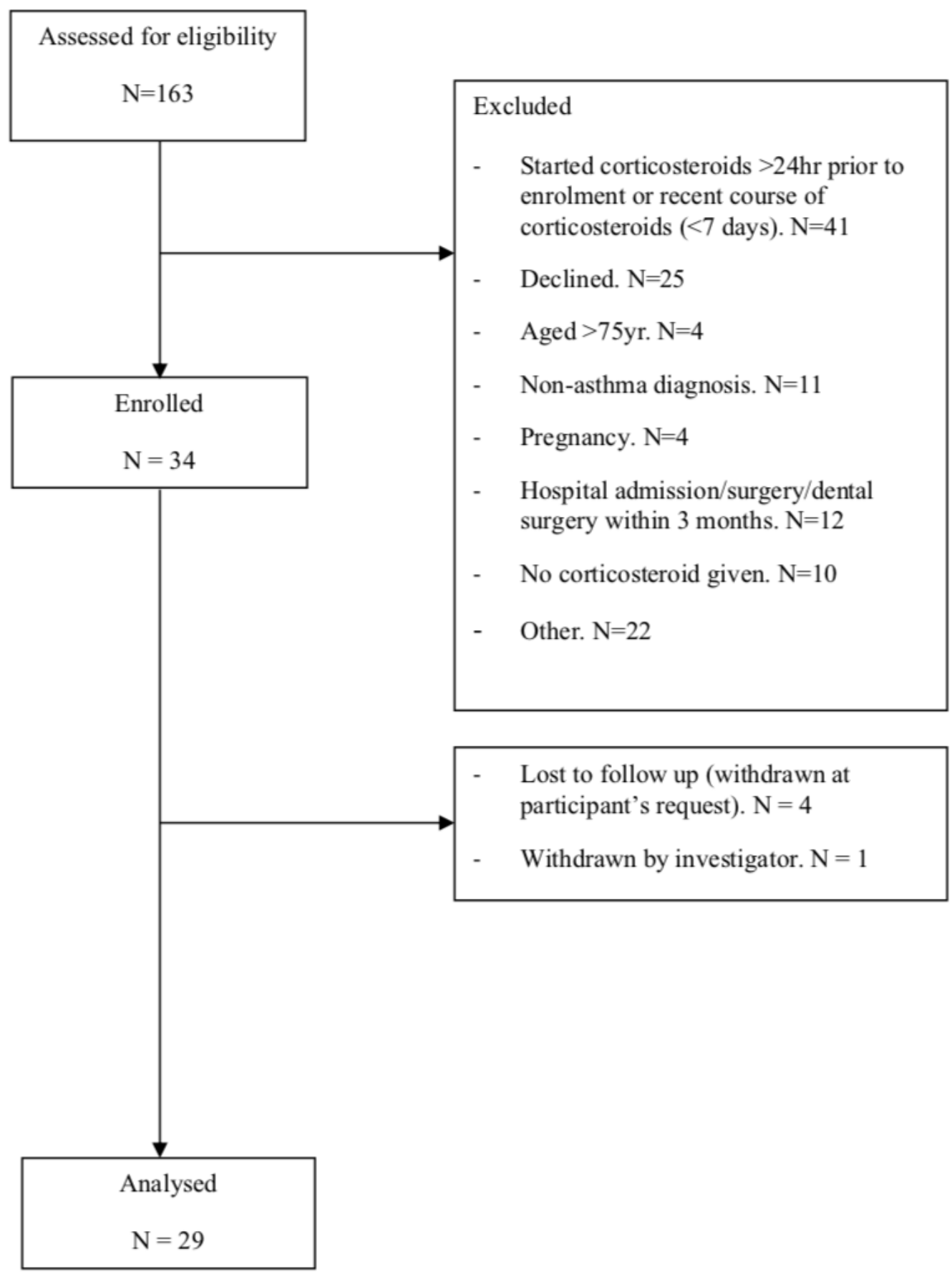


Table 4.2: Description of participants at enrolment

\begin{tabular}{|c|c|c|c|}
\hline Continuous Variable & Mean (SD) & Median (IQR) & Min to Max \\
\hline Age (years) $N=34$ & $39 \cdot 3(16 \cdot 7)$ & 35 (26 to 53$)$ & 18 to 70 \\
\hline BMI $\left(\mathrm{kg} / \mathrm{m}^{2}\right) \mathrm{N}=34$ & $34 \cdot 2(10 \cdot 8)$ & $30 \cdot 8(27 \cdot 1$ to $38 \cdot 6)$ & $20 \cdot 2$ to $67 \cdot 8$ \\
\hline $\mathrm{FEV}_{1}(\mathrm{~L}) \mathrm{N}=32$ & $2 \cdot 2(0.9)$ & $2.0(1.5$ to 2.8$)$ & 0.8 to 4 \\
\hline $\mathrm{FEV}_{1} \%$ predicted $\mathrm{N}=32$ & $77 \cdot 9(35 \cdot 1)$ & $75 \cdot 2(45 \cdot 4$ to 102$)$ & $20 \cdot 7$ to $149 \cdot 4$ \\
\hline $\mathrm{FVC}(\mathrm{L}) \mathrm{N}=32$ & $3 \cdot 3(1 \cdot 1)$ & $3.4(2.5$ to 3.9$)$ & 1.4 to 5.6 \\
\hline $\mathrm{FEV}_{1} / \mathrm{FVC}(\%) \mathrm{N}=32$ & $0.6(0.1)$ & $0.7(0.6$ to 0.7$)$ & 0.4 to 0.9 \\
\hline ACQ $-5 \mathrm{~N}=34$ & $3 \cdot 7(1 \cdot 2)$ & $3.8(3$ to $4 \cdot 6)$ & 1.6 to 5.8 \\
\hline $\mathrm{ICS}^{*}(\mu \mathrm{g}) \mathrm{N}=34$ & $450 \cdot 7(463 \cdot 3)$ & 400 (200 to 500$)$ & 0 to 2000 \\
\hline Periostin (ng/L) N=34 & $54 \cdot 6(21 \cdot 5)$ & $48 \cdot 6(42 \cdot 2$ to $62 \cdot 6)$ & 31.8 to 154.5 \\
\hline Eosinophil count $\left(\times 10^{9} / L\right) N=33$ & $0.07(0.12)$ & 0.01 (0 to 0.05$)$ & 0 to 0.46 \\
\hline $\operatorname{IgE}(\mathrm{U} / \mathrm{L}) \mathrm{N}=32$ & $660 \cdot 3(862 \cdot 5)$ & $341.5(122.5$ to 816$)$ & 4 to 3274 \\
\hline FeNO (ppb) N=32 & $48 \cdot 0(46 \cdot 1)$ & $33 \cdot 5$ (18 to 56$)$ & 7 to 195 \\
\hline Categorical Variable & N/34 (\%) & & \\
\hline Atopy & $27(79.4)$ & & \\
\hline Female & $23(67 \cdot 7)$ & & \\
\hline Current Smoker & $4(11 \cdot 8)$ & & \\
\hline European & $19(55 \cdot 9)$ & & \\
\hline Maori & $9(26 \cdot 5)$ & & \\
\hline Samoan & $2(5 \cdot 9)$ & & \\
\hline Other & $4(11 \cdot 8)$ & & \\
\hline
\end{tabular}

*Fluticasone-propionate equivalent dose

$\mathrm{SD}=$ standard deviation; $\mathrm{IQR}=$ interquartile range; $\mathrm{min}$ to $\mathrm{max}=$ minimum to maximum range; $\mathrm{BMI}=$ body mass index; $\mathrm{FEV} 1=$ forced expiratory volume in one second; $\mathrm{FVC}=$ forced vital capacity; $\mathrm{FeNO}=$ fractional exhaled nitric oxide; $\mu \mathrm{g}=$ microgram; $\mathrm{ng}=$ nanaogram; $\mathrm{ml}=$ millilitre; $\mathrm{L}=$ litre 
Peripheral blood eosinophils

Changes in peripheral blood eosinophils are shown in Figure 4.2. Mean (SD) peripheral blood eosinophils at week zero was $0.07(0.12) \times 10^{9} / \mathrm{L}$, which was significantly lower than at week 12 , mean $0.33(0.21) \times 10^{\%} / \mathrm{L}$, difference $(95 \% \mathrm{CI}) 0.25(0.15$ to $0 \cdot 36) \times 10^{9} / \mathrm{L}$, $\mathrm{P}<0.001$ (Table 4.3). There were no significant differences in peripheral blood eosinophils after week one compared with week 12 .

Figure 4.2: Boxplot of peripheral blood eosinophils by week after an exacerbation

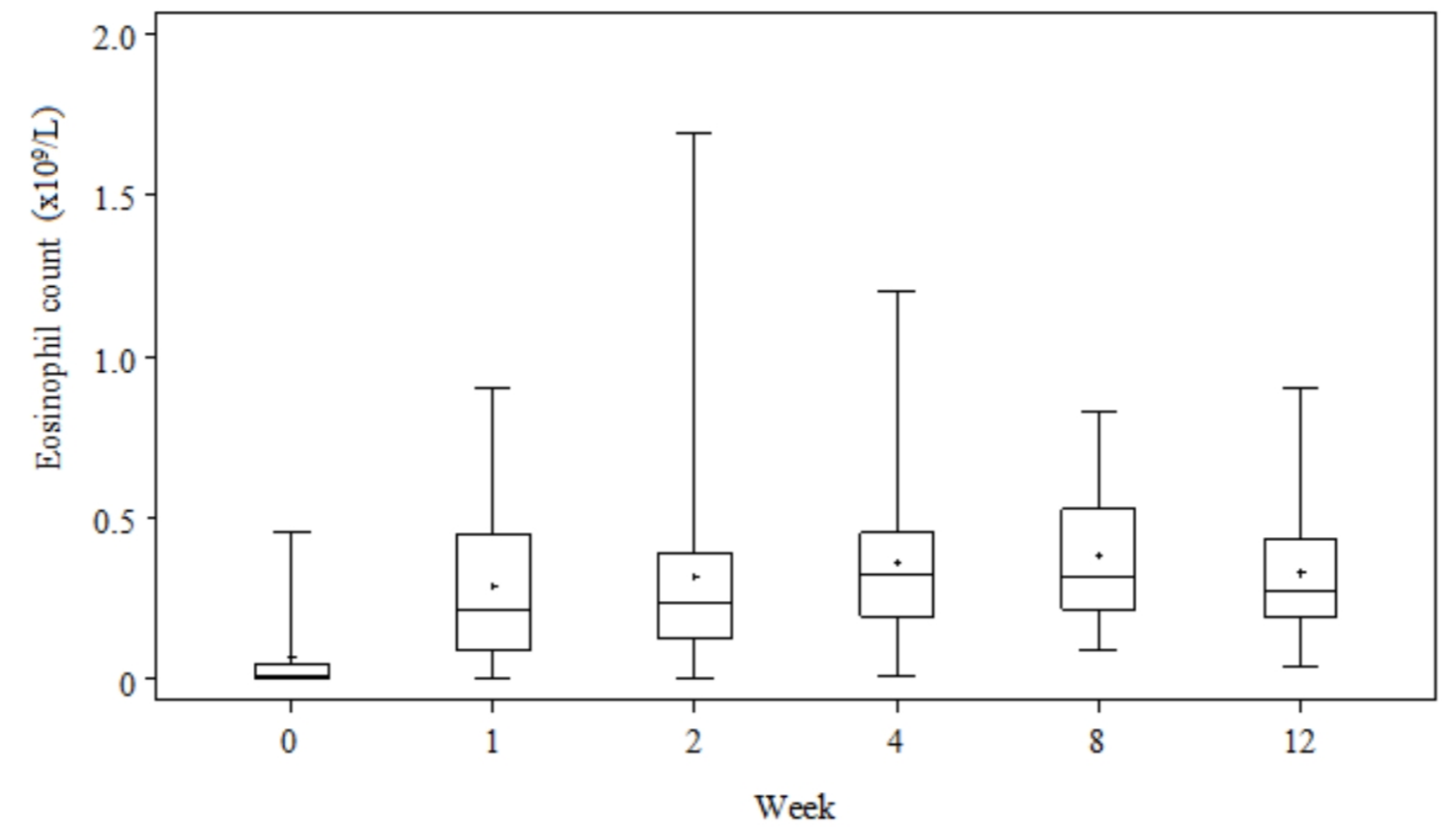

Symbol is the mean, the horizontal lines are the 25th, median, and 75th percentiles, and the whiskers extend from the minimum to maximum.

FeNO

Changes in FeNO are shown in Figure 4.3. The median (range) FeNO at week zero was 32 (7 to 195)ppb, similar to week 12, when the median (range) FeNO was 33 (10 to 188)ppb. The FeNO was about $30 \%$ lower at weeks one, two and four, with medians of 
22, 23 and 23ppb, respectively however these differences were not significant (Table 4.3). There were no significant differences in FeNO after week four compared with week 12.

Figure 4.3: Boxplot of Fractional exhaled Nitric Oxide (FeNO) by week after an exacerbation

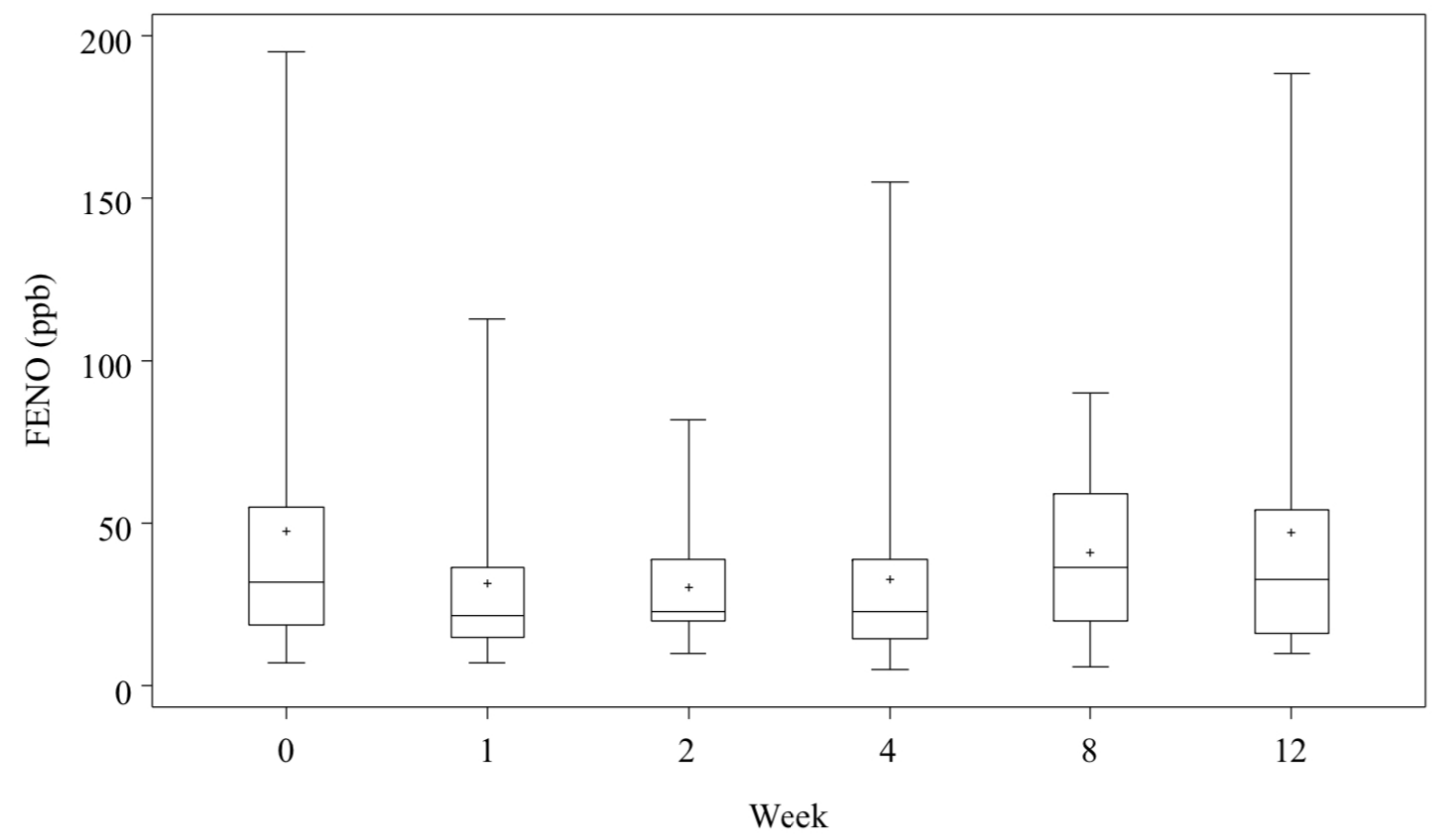

Symbol is the mean, the horizontal lines are the 25 th, median, and 75 th percentiles, and the whiskers extend from the minimum to maximum.

\section{Serum Periostin}

Changes in serum periostin are shown in Figure 4.4. The median (range) serum periostin at week zero was $48.9(31.8$ to 154.5$) \mathrm{ng} / \mathrm{ml}$, similar to week 12 , when the median (range) was $50.9(35.3$ to 98.3$) \mathrm{ng} / \mathrm{ml}$. At week one, the serum periostin level was lower than at week 12 , with a median (range) of $45.9(24.9$ to $146 \cdot 7) \mathrm{ng} / \mathrm{ml}$, ratio of 
geometric mean periostin $(95 \% \mathrm{Cl}) 0.86(0.82$ to 0.92), $\mathrm{P}<0.001$ (Table 4.3). After week four, serum periostin levels were not significantly different from week 12.

Figure 4.4: Boxplot of serum periostin by week after an exacerbation

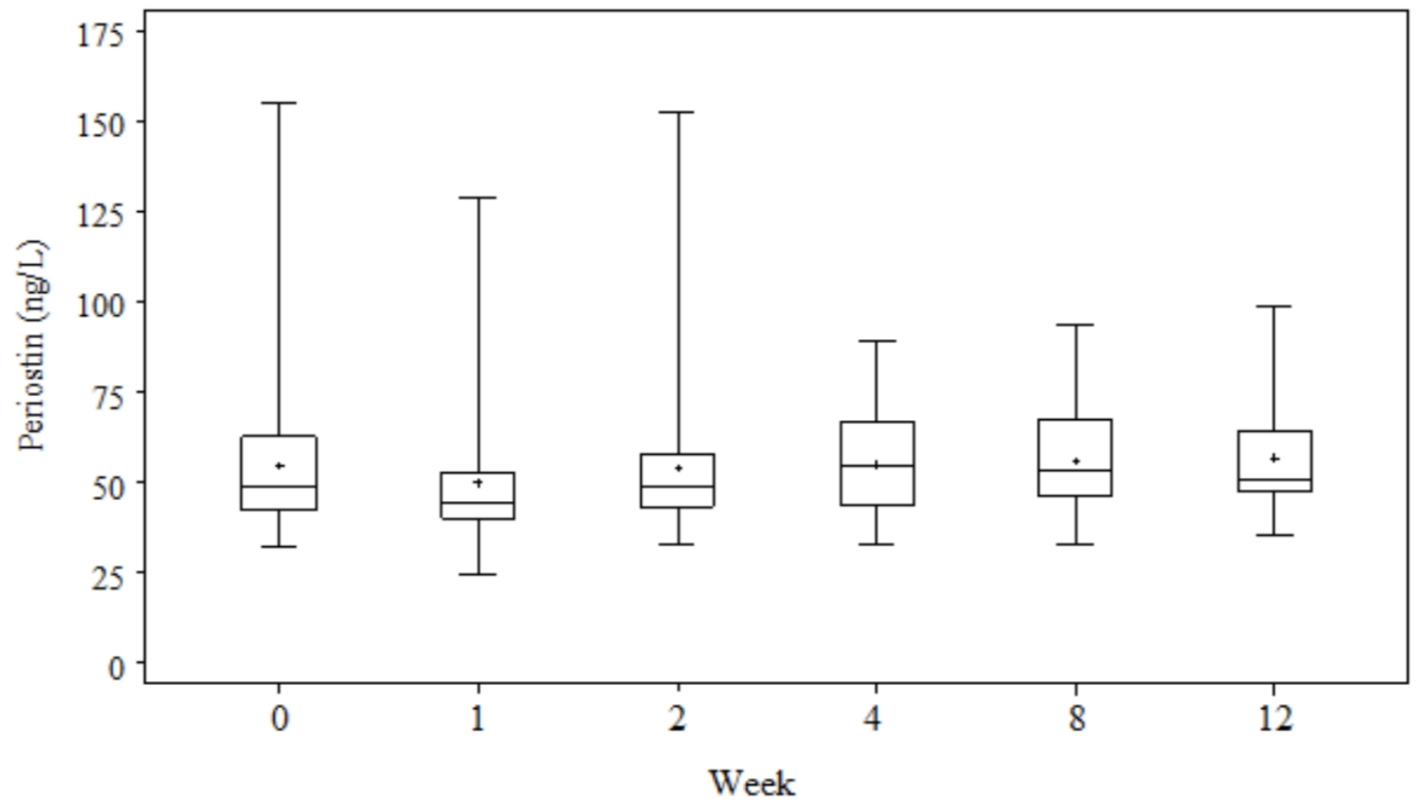

Symbol is the mean, the horizontal lines are the 25th, median, and 75th percentiles, and the whiskers extend from the minimum to maximum. 
Serum IgE

Changes in serum IgE are shown in Figure 4.5. Serum IgE levels were highest at week zero when the median (range) was 339 (4 to 3274)U/L, compared to week 12, when the median (range) was 249 ( 3 to 2451$) \mathrm{U} / \mathrm{L}$, ratio of geometric mean IgE ( $95 \% \mathrm{Cl}$ ) 1.32 (1.22 to 1.43), $\mathrm{P}<0.001$ (Table 4.3). After week two, serum IgE levels were not significantly different from week 12.

Figure 4.5: Boxplot of serum IgE by week after an exacerbation

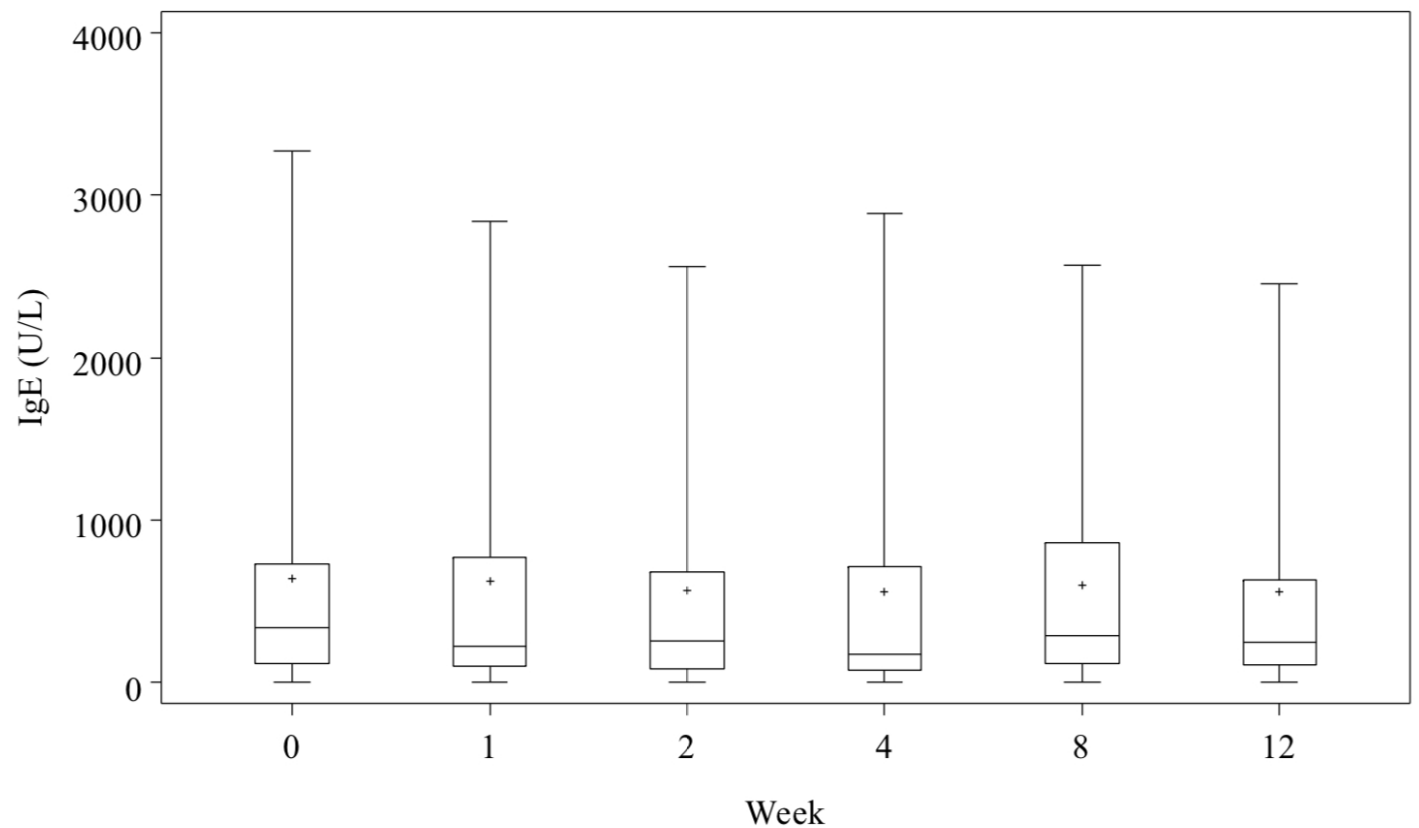

Symbol is the mean, the horizontal lines are the 25th, median, and 75th percentiles, and the whiskers extend from the minimum to maximum. 
Table 4.3: Comparison of Type 2 biomarkers with levels at week 12

\begin{tabular}{|c|c|c|c|c|c|}
\hline Type 2 variable & Weeks post & Mean (SD) & Min to Max & Difference from & $\mathbf{P}$ \\
\hline & exacerbation & & & week 12 (95\% CI) & \\
\hline \multirow[t]{6}{*}{$\begin{array}{l}\text { Blood eosinophils } \\
\left(\times 10^{9} / \mathrm{L}\right)\end{array}$} & $0 n=34$ & $\begin{array}{l}0.07 \\
(0 \cdot 12)\end{array}$ & 0 to 0.46 & $\begin{array}{l}0.25 \\
(0.15 \text { to } 0.36)\end{array}$ & $<0.001$ \\
\hline & $1 n=36$ & $\begin{array}{l}0.29 \\
(0 \cdot 24)\end{array}$ & 0 to 0.9 & $\begin{array}{l}0.06 \\
(-0.05 \text { to } 0.16)\end{array}$ & 0.31 \\
\hline & $2 n=29$ & $\begin{array}{l}0.32 \\
(0.34)\end{array}$ & 0 to 1.69 & $\begin{array}{l}-0.01 \\
(-0.12 \text { to } 0.11)\end{array}$ & 0.92 \\
\hline & $4 n=36$ & $\begin{array}{l}0.36 \\
(0 \cdot 25)\end{array}$ & 0.01 to 1.2 & $\begin{array}{l}-0.04 \\
(-0.15 \text { to } 0.06)\end{array}$ & 0.43 \\
\hline & $8 n=22$ & $\begin{array}{l}0 \cdot 38 \\
(0 \cdot 23)\end{array}$ & 0.09 to 0.83 & $\begin{array}{l}-0.07 \\
(-0.18 \text { to } 0.05)\end{array}$ & 0.25 \\
\hline & $12 n=21$ & $\begin{array}{l}0.33 \\
(0 \cdot 21)\end{array}$ & 0.04 to 0.9 & & \\
\hline Type 2 variable & $\begin{array}{l}\text { Weeks post } \\
\text { exacerbation }\end{array}$ & Median (IQR) & Min to Max & $\begin{array}{l}\text { Ratio of geometric } \\
\text { means }(95 \% \mathrm{Cl})\end{array}$ & $\mathbf{P}$ \\
\hline FeNO (ppb) & $0 n=33$ & $\begin{array}{l}32 \\
\text { (19 to } 55)\end{array}$ & 7 to 195 & $\begin{array}{l}1.13 \\
(0.90 \text { to } 1.43)\end{array}$ & $0 \cdot 28$ \\
\hline & $1 n=36$ & $\begin{array}{l}22 \\
(15 \text { to } 36 \cdot 5)\end{array}$ & 7 to 113 & $\begin{array}{l}0.82 \\
(0.65 \text { to } 1.03)\end{array}$ & 0.09 \\
\hline & $2 n=27$ & $\begin{array}{l}23 \\
\text { (20 to } 39)\end{array}$ & 10 to 82 & $\begin{array}{l}0.79 \\
(0.63 \text { to } 1.01)\end{array}$ & 0.06 \\
\hline
\end{tabular}


(Table 4.3 continued)

\begin{tabular}{|c|c|c|c|c|c|}
\hline & $4 n=36$ & $23 \cdot 0$ & 5 to 155 & $0 \cdot 87$ & $0 \cdot 21$ \\
\hline & & (14.5 to 39 ) & & (0.69 to 1.09 ) & \\
\hline & $8 n=22$ & $\begin{array}{l}36 \cdot 5 \\
(20 \text { to } 59)\end{array}$ & 6 to 90 & $\begin{array}{l}1.00 \\
(0.78 \text { to } 1.28)\end{array}$ & 0.99 \\
\hline & $12 n=22$ & $\begin{array}{l}33 \\
\text { (16 to } 54)\end{array}$ & 10 to 188 & & \\
\hline $\begin{array}{l}\text { Serum periostin } \\
\text { (ng/ml) }\end{array}$ & $0 \mathrm{n}=35$ & $\begin{array}{l}48 \cdot 9 \\
(42 \cdot 2 \text { to } 66 \cdot 6)\end{array}$ & $\begin{array}{l}31.8 \text { to } \\
154.5\end{array}$ & $\begin{array}{l}0.95 \\
(0.89 \text { to } 1.00)\end{array}$ & 0.06 \\
\hline & $1 n=36$ & $\begin{array}{l}45 \cdot 9 \\
40 \cdot 6 \text { to } 57 \cdot 1 \text { ) }\end{array}$ & $\begin{array}{l}24.9 \text { to } \\
146.7\end{array}$ & $\begin{array}{l}0.86 \\
(0.82 \text { to } 0.92)\end{array}$ & $<0.001$ \\
\hline & $2 n=29$ & $\begin{array}{l}48 \cdot 7 \\
(43 \cdot 3 \text { to } 56 \cdot 8)\end{array}$ & $\begin{array}{l}32 \cdot 6 \text { to } \\
152 \cdot 2\end{array}$ & $\begin{array}{l}0.93 \\
(0.87 \text { to } 0.98)\end{array}$ & 0.008 \\
\hline & $4 n=36$ & $\begin{array}{l}52 \cdot 9 \\
(43 \cdot 1 \text { to } 67 \cdot 6)\end{array}$ & $32 \cdot 1$ to $90 \cdot 4$ & $\begin{array}{l}0.98 \\
(0.93 \text { to } 1.04)\end{array}$ & 0.49 \\
\hline & $8 n=23$ & $\begin{array}{l}52 \cdot 9 \\
(45 \cdot 9 \text { to } 65)\end{array}$ & $32 \cdot 9$ to $93 \cdot 9$ & $\begin{array}{l}1.02 \\
(0.96 \text { to } 1.08)\end{array}$ & 0.57 \\
\hline & $12 n=21$ & $\begin{array}{l}50 \cdot 9 \\
(47 \cdot 7 \text { to } 64 \cdot 3)\end{array}$ & $35 \cdot 3$ to $98 \cdot 3$ & & \\
\hline
\end{tabular}


(Table 4.3 continued)

\begin{tabular}{|c|c|c|c|c|c|}
\hline \multirow[t]{6}{*}{ Serum IgE (U/L) } & $0 n=33$ & $\begin{array}{l}339 \\
\text { (120 to } 729)\end{array}$ & 4 to 3274 & $\begin{array}{l}1 \cdot 32 \\
(1 \cdot 22 \text { to } 1 \cdot 43)\end{array}$ & $<0.001$ \\
\hline & $1 n=35$ & $\begin{array}{l}227 \\
\text { (98 to } 772 \text { ) }\end{array}$ & 5 to 2841 & $\begin{array}{l}1 \cdot 22 \\
(1 \cdot 14 \text { to } 1 \cdot 32)\end{array}$ & $<0.001$ \\
\hline & $2 n=28$ & $\begin{array}{l}254 \\
\text { (82 to } 677 \text { ) }\end{array}$ & 4 to 2561 & $\begin{array}{l}1.04 \\
(0.96 \text { to } 1.12)\end{array}$ & $0 \cdot 30$ \\
\hline & $4 n=36$ & $\begin{array}{l}175 \cdot 5 \\
(80 \text { to } 712 \cdot 5)\end{array}$ & 4 to 2887 & $\begin{array}{l}1.00 \\
(0.93 \text { to } 1.09)\end{array}$ & 0.91 \\
\hline & $8 n=22$ & $\begin{array}{l}285 \cdot 5 \\
\text { (119 to } 862 \text { ) }\end{array}$ & 4 to 2572 & $\begin{array}{l}1.04 \\
(0.96 \text { to } 1.12)\end{array}$ & $0 \cdot 31$ \\
\hline & $12 n=21$ & $\begin{array}{l}249 \\
\text { (109 to 629) }\end{array}$ & 3 to 2451 & & \\
\hline
\end{tabular}


Lung function

The mean (SD) $\mathrm{FEV}_{1}$ percent predicted at week zero was $77.9(35 \cdot 1) \%$, significantly lower than at week 12, when the mean (SD) was 90.4 (35)\%, difference (95\% Cl) 13.2 (8 to 18.3), $\mathrm{P}<0 \cdot 001$. After one week there was no significant difference in $\mathrm{FEV}_{1}$ percent predicted when compared with 12 weeks after the exacerbation. (Table 4.4).

Table 4.4: Comparison of FEV1 \% predicted values with Week 12

\begin{tabular}{llll}
\hline Week & $\begin{array}{l}\text { Least squares mean } \\
(95 \% \mathrm{Cl})\end{array}$ & Week 12 Comparison & P value \\
\hline 0 & $79.1(65.3$ to 93.0$)$ & $13.2(8.0$ to 18.3$)$ & $<0.001$ \\
1 & $87.8(73.5$ to 102.1$)$ & $4.5(-0.6$ to 9.7$)$ & 0.083 \\
2 & $90.5(76.6$ to 104.5$)$ & $1.8(-3.5$ to 7.0$)$ & 0.51 \\
4 & $88.1(74.2$ to 102.1$)$ & $4.2(-0.9$ to 9.2$)$ & 0.11 \\
8 & $88.1(74.0$ to 102.2$)$ & $4.2(-1.3$ to 9.6$)$ & 0.13 \\
12 & $92.3(78.2$ to 106.4$)$ & & \\
\hline
\end{tabular}




\subsubsection{Discussion}

This study found that the time course of the changes in peripheral blood eosinophils, FeNO, serum periostin and serum IgE all varied during and after treatment for a severe exacerbation of asthma. Peripheral blood eosinophils were the first to fall after treatment with systemic corticosteroids (within 24 hours), followed by serum periostin (after one week), and FeNO (after two weeks). In contrast the initial serum IgE measurement was higher than the reference week 12 value. All the biomarkers achieved stable levels within four weeks after the start of a severe exacerbation, indicating there is a four week period during and after a severe exacerbation where biomarker measurements are influenced by the event and its treatment. This is of clinical relevance if these biomarkers are to be used to identify responders to monoclonal antibody therapy directed against IgE, IL4Ra, IL-5 and IL-13 in asthma.

Peripheral blood eosinophils were almost fully suppressed within 24 hours of commencing systemic corticosteroids for an exacerbation, with a mean value of $0.07 \times 10^{9} / \mathrm{L}$, compared with the week 12 measurement of $0.33 \times 10^{9} / \mathrm{L}$. It is likely that their levels were suppressed by treatment with systemic corticosteroid, an effect which has been observed within five hours in non-asthmatic adults, with levels returning to pre-treatment levels within 24 hours of stopping corticosteroid therapy. ${ }^{210}$ This latter observation is consistent with our finding that blood eosinophil levels did not differ from the reference week 12 measurements after week two, as the average length of the course of corticosteroids was 11 days. In the USA, one of the current indications for use of mepolizumab, a monoclonal antibody directed against IL-5, is a 
blood eosinophil count of greater than $0 \cdot 3 \times 10^{9} /$ L. ${ }^{211}$ In our cohort, there were $12 / 29$ (41\%) with week 12 measurements who would have met this criteria, compared with only $3 / 34(9 \%)$ at the initial 24 hour measurement. The marked suppression of blood eosinophils during systemic corticosteroid treatment for a severe exacerbation suggests that reference should not be made to blood eosinophil levels taken during this period, for the purpose of assessing potential response to monoclonal antibody therapy directed against IL-5.

There was a trend for FeNO levels to fall by about $30 \%$ at week one, where they remained at this level until week four. This is consistent with previous studies which demonstrated that FeNO is suppressed by treatment with both inhaled ${ }^{212,213}$ and systemic corticosteroids. ${ }^{214}$ In steroid naïve, mild asthmatics, the use of ICS have been shown to cause a dose-dependent fall in FeNO of up to $30 \%$ one week after the start of treatment, ${ }^{212}$ similar to the effect in severe asthmatics on high dose ICS treated with systemic corticosteroids. ${ }^{214}$ In the study of mild asthmatics, FeNO levels returned to baseline two weeks after stopping ICS treatment, a time course similar to our findings in relation to systemic corticosteroid treatment. The use of FeNO is not currently recommended as a predictor of response to monoclonal antibody therapy directed against IL-5 or IgE. However, FeNO is a useful maker of responsiveness to inhaled corticosteroid therapy, ${ }^{91}$ and reference to values taken within four weeks of a severe exacerbation may underestimate the potential benefit of an increase in maintenance inhaled corticosteroid dose. 
There was a modest $10 \%$ fall in serum periostin at weeks one and two, similar in magnitude to the response seen previously to treatment with inhaled ${ }^{100}$ and systemic ${ }^{171}$ corticosteroids in adults with stable asthma. Periostin levels greater than the proposed $50 \mathrm{ng} / \mathrm{ml}$ cut point (using the Elecsys ${ }^{\circledR}$ Periostin assay) have been shown to predict response to monoclonal antibody therapy directed against IL-13 $3^{53}$ and IgE. ${ }^{69}$ However, serum periostin was suppressed to a median level of $45.9 \mathrm{ng} / \mathrm{ml}$ at week one, compared with the median baseline level of $50.9 \mathrm{ng} / \mathrm{ml}$ at week 12 , and remained below the $50 \mathrm{ng} / \mathrm{ml}$ cut point until four weeks after the start of the exacerbation. Therefore it would be preferable not to use serum periostin levels measured in the first four weeks after an exacerbation of asthma for predicting responsiveness to the monoclonal antibody therapies mentioned above.

A different pattern was observed with serum IgE, which was about $25 \%$ higher at week zero before falling to the reference level at week two. There are a number of potential mechanisms that might contribute to this pattern. Firstly it may relate to the use of systemic corticosteroids which cause a paradoxical stimulation of polyclonal IgE release from B-lymphocytes, ${ }^{215}$ an effect which has been demonstrated in asthmatics in vivo. ${ }^{216}$ Alternatively beta-agonist therapy administered during the exacerbation may have increased IgE production through stimulation of IL-4. ${ }^{217}$ Another possibility is that IgE levels were elevated as a result of allergen exposure which may have contributed to some of the exacerbations. ${ }^{218}$ Although IgE levels are not predictive of response to anti-lgE therapy, ${ }^{96}$ a total serum IgE range of between 30 and $700 \mathrm{U} / \mathrm{L}^{219}$ or 30 and $1500 \mathrm{U} / \mathrm{L}^{203}$ is required before initiating treatment in the UK and EU respectively. As a 
result, a delay of at least two weeks after treatment for an exacerbation is preferred to assess for potential eligibility for anti IgE therapy in asthma.

The different time courses of the changes in biomarkers was not surprising, due to their different nature, ranging from a specific protein, antibody and cell in circulating blood to an expired gas. The biomarkers have different pathways for synthesis and migration between vascular and tissue compartments, lifespans and responses to steroid treatment.

In terms of methodological limitations, our study was designed to detect a difference in serum periostin and it is likely that the sample size was too small to detect a meaningful change with respect to FeNO, which has greater intra-participant variability compared with periostin (mean coefficient of variation of $19 \cdot 8 \%$ vs $5 \cdot 0 \%$ ). ${ }^{53}$ We have carried out a large number of statistical tests and some of the statistically significant associations may be a result of Type 1 error rate inflation. Baseline measurements of the Type 2 biomarkers and lung function during a stable disease phase prior to the exacerbation were not obtained, due to the study design. We therefore compared all periostin levels to those taken 12 weeks after the exacerbation under the assumption that stable levels would be attained by then. As no previous research has been conducted assessing the variability of Type 2 biomarkers during an exacerbation, further robust clinical studies are required to corroborate our findings. Finally, the underlying cause of the asthma exacerbations were heterogeneous. Different triggers (viral, allergen or bacterial) can initiate different inflammatory cascades, ${ }^{18}$ but this 
study was not powered to analyse the difference in response between these subgroups.

In conclusion, peripheral blood eosinophils, FeNO, serum periostin and serum IgE all have different time courses and magnitude of change during and following treatment for a severe exacerbation of asthma. Therefore we suggest a delay of up to four weeks following a severe exacerbation is required if these biomarkers are used to determine eligibility for, or predicting responsiveness to, monoclonal antibody therapy. 


\subsection{Study Six: Type 2 biomarkers and prediction of future exacerbations in asthma}

\subsubsection{Introduction}

The Type 2 biomarkers blood eosinophils, FeNO, serum periostin and serum IgE are predictors of responsiveness to monoclonal antibody therapy directed against IL-4R $\alpha$, IL-5, IL-13 and IgE. In studies primarily undertaken in patients with severe eosinophilic asthma, higher levels of blood eosinophils, FeNO and serum periostin have also been shown to predict an increased risk of severe exacerbations. Whether blood eosinophils, FeNO or serum periostin predict severe exacerbations in a broader asthma population is not known.

\subsubsection{Hypothesis}

High levels of Type 2 biomarkers are predictive of future severe exacerbations.

\subsubsection{Objectives}

To describe the association between the Type 2 biomarkers, blood eosinophils, FeNO, serum periostin, serum IgE and time to a severe exacerbation in a broad asthma population. 


\subsubsection{Methods}

\section{Study Population}

This study followed-up participants from two previous observational studies conducted by the MRINZ. The New Zealand Respiratory Health Survey (NZRHS) was a three-phase cross-sectional study, that enrolled 451 subjects from a randomly selected population of adult asthmatics between November 2010 and August 2012, from which 285 had a doctor's diagnosis of asthma with complete data. ${ }^{220}$ The second study enrolled 60 adults with stable asthma, on ICS and LABA therapy, recruited between March 2014 and February 2015. ${ }^{171} \mathrm{~A}$ total of 15 participants were enrolled in both studies, so the baseline data used was from their first visit with complete data. For each study, participants had baseline measures of lung function, FeNO levels, blood eosinophil count, serum periostin levels and total serum IgE. Participants were invited to reattend the outpatient facility at the MRINZ for a single follow up visit, at least 12 months after their baseline measurements, which took place from May 2015 to June 2016.

Inclusion and Exclusion criteria

Follow up visits were rescheduled if participants reported sustaining a bone fracture, undergoing major general or dental surgery, or taking systemic corticosteroids within three months of the study visit. Additionally, visits were rescheduled if participants reported an upper or lower respiratory tract infection in the three weeks prior to the 
study visit. Participants were excluded from follow up if they were pregnant or breastfeeding.

Ethical approval for this study was given by the New Zealand Ethics Committee (15/CEN/33). The study was prospectively registered with the Australia New Zealand Trial Registry (ACTRN12615000503527). All participants provided written informed consent prior to participation.

\section{Clinical Data}

Respiratory history

All participants were asked about severe exacerbations since the baseline visit. A severe exacerbation was identified in accordance with the American Thoracic Society (ATS) and European Respiratory Society (ERS) criteria, ${ }^{209}$ requiring the use of systemic corticosteroids (tablets, suspension or injection), or an increase from a stable maintenance dose, for at least three days or a hospitalisation or Emergency Department visit because of asthma requiring systemic corticosteroids. Courses of corticosteroids separated by more than a week were treated as separate severe exacerbations. Patient-reported exacerbations, including date, treatment provided and healthcare provider details, were recorded in an investigator-led questionnaire and then corroborated with general practioner (GP) and hospital electronic medical records. 
A patient-reported severe exacerbation was counted if there was a prescription of systemic corticosteroids for three or more days for the treatment of asthma in the patient's medical records, in concordance with the date provided by the patient. In the case of patient-reported self-medication, where patients followed an existing acute management plan of taking systemic corticosteroids from a home rescue pack, GP medical records were checked for appointments or prescriptions where the rescue pack medication would have been replaced. In both cases, if there was no evidence of prescriptions for systemic corticosteroid, the patient-reported event was discounted. Regarding occasions where the severe exacerbation occurred abroad and no supporting documentation was accessible, the patient-reported exacerbations were counted. In the event that GP or hospital records were not available, all patientreported exacerbations were counted.

The remaining questionnaires used were the Asthma Control Questionnaire (ACQ) and the Asthma Quality of Life Questionnaire for Standardised activities (AQLQ-S) to establish respiratory health status and disease control.

\section{Lung function}

Spirometry was performed using a Masterscreen Pneumo (Masterscreen Version 2.0, Carefusion, Hoechberg, Germany), in accordance ATS guidelines ${ }^{184}$. Global Lung Initiative reference ranges were used ${ }^{185}$. 


\section{Biomarkers}

Blood was drawn for full blood count and differential (Sysmex platform, Mundelein, USA), creatinine and electrolytes and serum IgE (Roche, Cobas 501, NZ), which were performed immediately in the local laboratory. Blood for serum periostin measurement was coagulated, centrifuged and serum aliquots stored at $-80^{\circ} \mathrm{C}$ for analysis using the Elecysys ${ }^{\circledast}$ periostin immunoassay (Roche Diagnostics, Penzberg, Germany) described previously ${ }^{115}$. FeNO was measured using a NIOX MINO monitor (Aerocrine AB, Solna, Sweden) in accordance with ATS guidelines ${ }^{89}$.

\section{Study Size}

In total, 330 participants were invited to take part in the study where the assumed attrition rate would be in the order of $30 \%$, anticipating a total of 230 participants would be enrolled. We estimated that this sample size would have $95 \%$ confidence intervals for a proportion of $20 \%$ (the anticipated proportion of participants with a severe exacerbation) of plus or minus $5 \%$.

\section{Statistical Methods}

Multivariate models, adjusting for the participant's original cohort and whether or not they were on ICS at baseline, were used to estimate the relative differences in time to a severe exacerbation by estimating the Hazard Ratio between the Type 2 biomarkers and time to severe exacerbation using the Cox Proportional Hazards model. In the analysis lower hazard ratio corresponds with a longer time to a severe exacerbation. 
Comparison of Type 2 biomarkers was made between those who had a severe exacerbation and those who did not, using t-tests. The predictor variables FeNO, serum periostin and serum IgE required logarithm transformation to better meet normal distribution. A ratio of geometric means was used to describe the exponent of these variables. A multiplier of 0.693 represents a doubling of the geometric mean.

Linear regression models were used to describe the relationship between baseline Type 2 biomarkers and change in $\mathrm{FEV}_{1}$, including multivariate analysis adjusting for age, sex, ICS use and smoking status at baseline. A significant interaction term suggested that the change in $\mathrm{FEV}_{1}$ with time was dependent of the Type 2 biomarker.

SAS 9.4 was used. 


\subsubsection{Results}

A total of 212 out of the potential 330 participants returned for testing, of which 177 (84\%) were from the New Zealand Health Respiratory Survey (NZRHS). ${ }^{220}$ The median (range) time between baseline and follow up visits was 3.8 (1.1 to 5.3) years, with a total of 642 patient years of follow-up. The flow of participants through the study is shown in Figure 4.6. All $(212 / 212 ; 100 \%)$ hospital electronic medical records were reviewed to corroborate hospital attendances and prescriptions of systemic corticosteroids. A total of 22/212 (10\%) participants did not have GP electronic records reviewed by an investigator as permission was not granted by GP and 4/212 (2\%) participants had severe exacerbations outside of New Zealand, which could not be corroborated locally. There were $67 / 212$ (32\%) participants with at least one severe exacerbation of asthma. There were a total of 189 severe exacerbations, representing a rate of 0.29 severe exacerbations per patient year. The participants had well controlled asthma of mild to moderate severity (Table 4.5). At baseline/follow-up, there were $41 / 38$ participants prescribed no inhalers, $16 / 37$ participants prescribed a short-acting beta-agonist (SABA) only, 63/36 prescribed an ICS with SABA inhaler and 92/101 participants prescribed an ICS with a LABA. No participants were prescribed maintenance systemic corticosteroids for their asthma, although at follow-up three people reported the chronic use of oral corticosteroids for non-asthma related conditions. The mean scores at baseline for the ACQ-5 and AQLQ-S were 0.91 and 6.13 , respectively. The mean $\mathrm{FEV}_{1}$ percent predicted was $88.9 \%$ and $87.3 \%$ at baseline and follow up visits, respectively. 
Figure 4.6: Flow of participants through Study Six

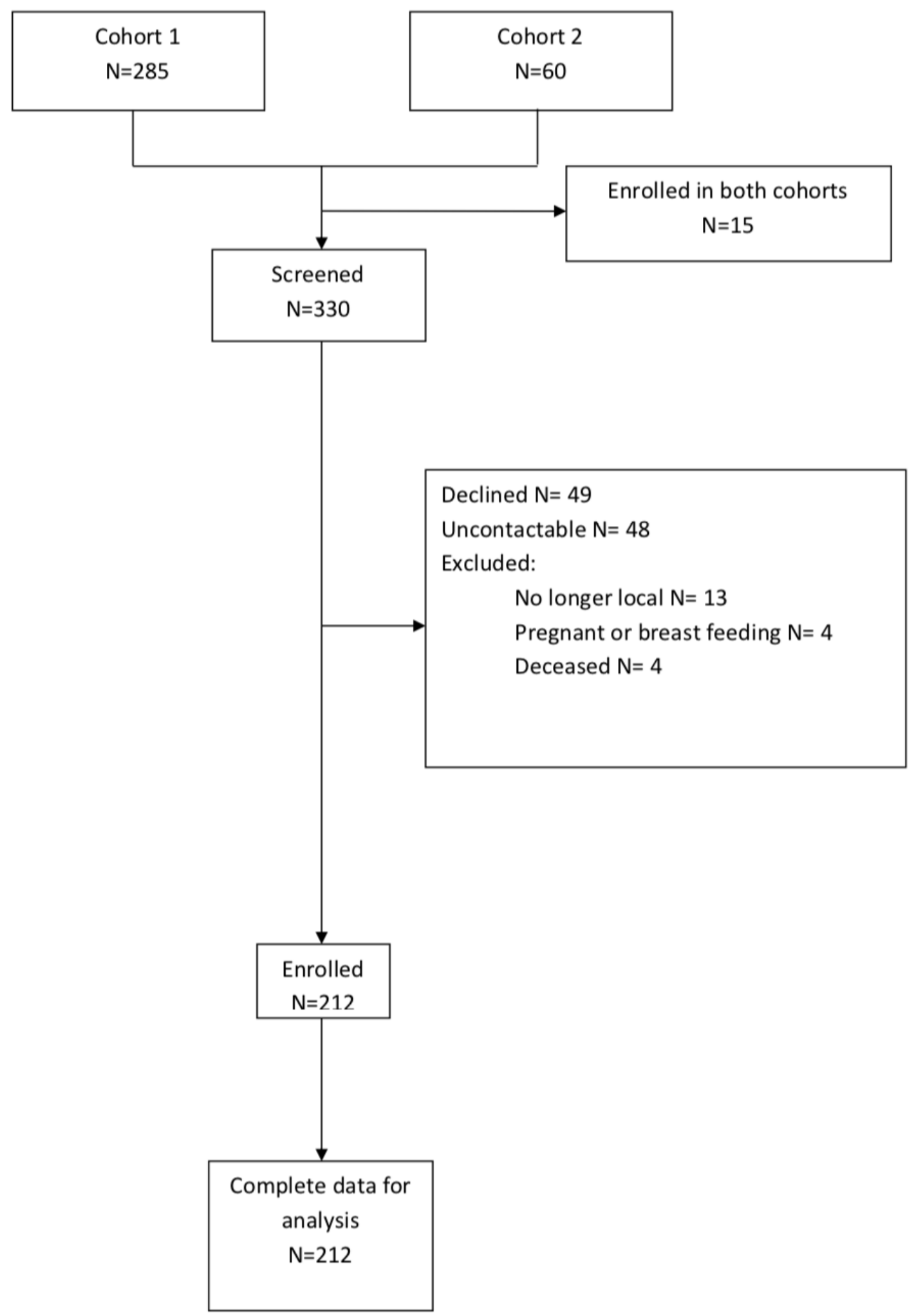


Table 4.5: Participant characteristics for Study Six

\begin{tabular}{|c|c|c|c|}
\hline \multirow[t]{2}{*}{ Continuous Variable } & Mean (SD) & Mean (SD) & Difference* (SD) \\
\hline & Baseline & Follow up & \\
\hline Age (years) & $50.5(13.2)$ & & \\
\hline BMI (kg/m2) & $29(6.1)$ & & \\
\hline ACQ-5 & $0.91(0.74)$ & $0.73(0.76)$ & $-0.17(0.71)$ \\
\hline AQLQ-S & $6.13(0.92)$ & $6.23(0.81)$ & $0.09(0.75)$ \\
\hline FEV1 (L) & $2.90(0.91)$ & $2.77(0.87)$ & $-0.14(0.34)$ \\
\hline FEV1 \% predicted (\%) & $88.9(19.8)$ & $87.3(18.7)$ & $-1.6(10.2)$ \\
\hline $\begin{array}{l}\text { Blood eosinophils } \\
\left(\times 10^{9} / \mathrm{L}\right)\end{array}$ & $0.25(0.19)$ & $0.24(0.18)$ & $-0.01(0.15)$ \\
\hline $\mathrm{FeNO}(\mathrm{ppb})^{+}$ & 22.5 (13.6 to 44.4$)$ & 22 (15 to 33 ) & $-0.4(-9.5$ to 5$)$ \\
\hline Serum periostin $(\mathrm{ng} / \mathrm{ml})^{+}$ & $52.8(45$ to 65.5$)$ & 52.6 (43.7 to 61.7 ) & $-2.24(-8.1$ to 4.4$)$ \\
\hline Serum IgE $(I U / L)^{+}$ & 123 (32 to 262.5 ) & 103 (23 to 243 ) & $-8(-74.8$ to 8$)$ \\
\hline Categorical variables & N/212 (\%) & $\mathrm{N} / 212(\%)$ & Difference $(\mathrm{N})$ \\
\hline \multicolumn{4}{|l|}{ Ethnicity } \\
\hline Asian & $8(3.8)$ & $8(3.8)$ & \\
\hline European & $189(89.2)$ & $189(89.2)$ & \\
\hline Maori & $8(3.8)$ & $8(3.8)$ & \\
\hline Pacific & $5(2.4)$ & $5(2.4)$ & \\
\hline Other & $2(0.9)$ & $2(0.9)$ & \\
\hline Female & 109 (51.4) & 109 (51.4) & \\
\hline Current smoker & $13(6.1)$ & $13(6.1)$ & 0 \\
\hline ICS use & 155 (74.1) & $136(64.1)$ & -19 \\
\hline
\end{tabular}

${ }^{*}$ Calculated as Follow up visit value minus baseline visit value. † Medians (IQR) given here as variables skewed. 
Association between Type 2 variables and time to severe exacerbation

The associations between Type 2 biomarkers and time to severe exacerbation are shown in Table 4.6. The multivariate associations are adjusted for which recruitment cohort the participants were from and for use of ICS or not. There was no statistically significant evidence of an association between the cohort the participants were from and time to severe exacerbation in any of the multivariate analyses. In the univariate analysis the hazard ratio $(95 \% \mathrm{Cl})$ for time to exacerbation was consistent with the larger original cohort being less at risk: 0.40 (0.21 to 0.85 ). However in all the multivariate models this estimate of association, once adjusted for whether on ICS or not and the individual Type 2-related measurement, the point estimates for associations had increased to 0.55 with wide confidence intervals ( 0.28 to 1.17 ). 
Table 4.6: Association between Type 2 biomarkers and time to severe exacerbation

\begin{tabular}{|c|c|c|c|c|}
\hline \multirow[t]{2}{*}{ Type 2 biomarkers } & \multicolumn{2}{|l|}{ Univariate } & \multicolumn{2}{|l|}{ Multivariate } \\
\hline & $\begin{array}{l}\text { Hazard Ratio (95\% } \\
\mathrm{Cl)}\end{array}$ & $P$ value & $\begin{array}{l}\text { Hazard Ratio (95\% } \\
\mathrm{Cl)}\end{array}$ & $P$ value \\
\hline $\begin{array}{l}\text { Per } 0.1 \text { unit } \\
\text { increase in blood } \\
\text { eosinophils }\end{array}$ & $0.92(0.79$ to 1.07$)$ & 0.28 & 0.89 (0.76 to 1.05$)$ & 0.17 \\
\hline $\begin{array}{l}\text { Per } 0.693 \text { unit } \\
\text { increase of log } \\
\text { FeNO }\end{array}$ & 0.64 (0.52 to 0.79$)$ & $<0.001$ & 0.65 (0.52 to 0.81$)$ & $<0.001$ \\
\hline $\begin{array}{l}\text { Per } 0.693 \text { unit } \\
\text { increase of log } \\
\text { serum periostin }\end{array}$ & 0.59 (0.33 to 1.07$)$ & 0.08 & 0.62 (0.35 to 1.09$)$ & 0.10 \\
\hline $\begin{array}{l}\text { Per } 0.693 \text { unit } \\
\text { increase of log } \\
\text { serum IgE }\end{array}$ & $0.93(0.83$ to 1.04$)$ & 0.20 & 0.89 (0.80 to 1.00$)$ & 0.05 \\
\hline
\end{tabular}

Peripheral blood eosinophils

Mean (SD) blood eosinophil counts were $0.25(0.19) \times 10^{\%} / \mathrm{L}$ and $0.24(0.18) \times 10^{\%} / \mathrm{L}$, at baseline and follow up visits respectively. There was no significant association between baseline blood eosinophil count and time to severe exacerbation. The adjusted Hazard Ratio $(95 \% \mathrm{Cl})$ for association between higher eosinophil count and time to exacerbation was 0.89 (0.76 to 1.05$)$ per $0.1 \times 10^{9} / \mathrm{L}$ eosinophil count increase, $\mathrm{P}=0.17$ (Figure 4.7A).

FeNO

Median (interquartile range; IQR) FeNO levels were 22.5 (13.6 to 44.4) ppb and 22 (15 to 33) ppb, at baseline and follow up visits respectively. The analysis of FeNO was on the logarithm transformed scale. The adjusted Hazard Ratio $(95 \% \mathrm{Cl})$ for association 
between baseline log FeNO and time to severe exacerbation was 0.65 ( 0.52 to 0.81 ), per $0.693 \mathrm{ppb}$ of log FeNO increase, $\mathrm{P}<0.001$ (Figure 4.7B).

Serum periostin

Median (IQR) serum periostin levels were 52.8 (45 to 65.5$) \mathrm{ng} / \mathrm{ml}$ and 52.6 (43.7 to 61.7) $\mathrm{ng} / \mathrm{ml}$, at baseline and follow up visits respectively. The analysis of serum periostin was on the logarithm transformed scale. The adjusted Hazard Ratio $(95 \% \mathrm{Cl})$ for association between baseline log serum periostin and time to severe exacerbation was 0.62 (0.35 to 1.09 ) per $0.693 \mathrm{ng} / \mathrm{ml}$ of log periostin increase, $\mathrm{P}=0.1$ (Figure $4.7 \mathrm{C}$ ).

Serum IgE

Median (IQR) serum IgE levels were 123 (32 to 262.5) IU/L and 103 (28 to 243) IU/L, at baseline and follow up visits respectively. The analysis of serum IgE was on the logarithm transformed scale. The adjusted Hazard Ratio $(95 \% \mathrm{Cl})$ for association between baseline log serum IgE and time to severe exacerbation was 0.89 (0.80 to 1.00) per $0.693 \mathrm{IU} / \mathrm{L}$ of log IgE increase, $\mathrm{P}=0.05$ (Figure 4.7D).

ICS use at baseline

There was strong evidence of an association between ICS use at baseline and time to severe exacerbation. In all the models ICS use was associated with an increased risk of exacerbation. The univariate hazard ratio $(95 \% \mathrm{Cl})$ was $3.86(1.76$ to 8.48$)$ and the adjusted hazard ratio $(95 \% \mathrm{Cl})$ was 3.66 (1.65 to 8.08$)$. 
Figure 4.7: Kaplan-Meier survival curves for quartiles of blood eosinophil count (A), $\log$ FeNO (B), log serum periostin (C) and log serum IgE (D)

A

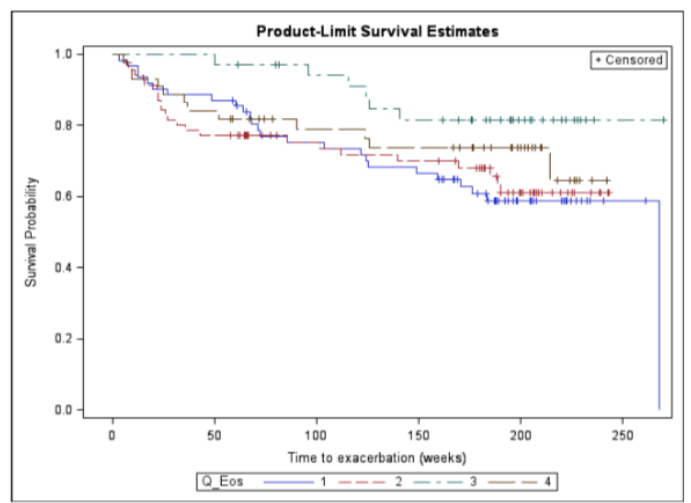

B

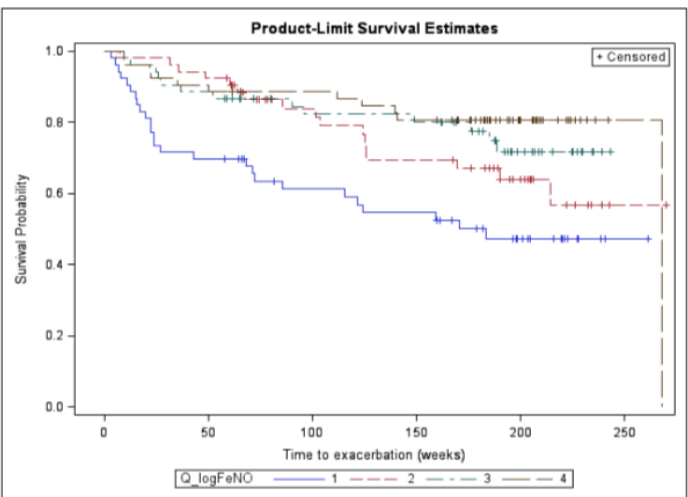

D
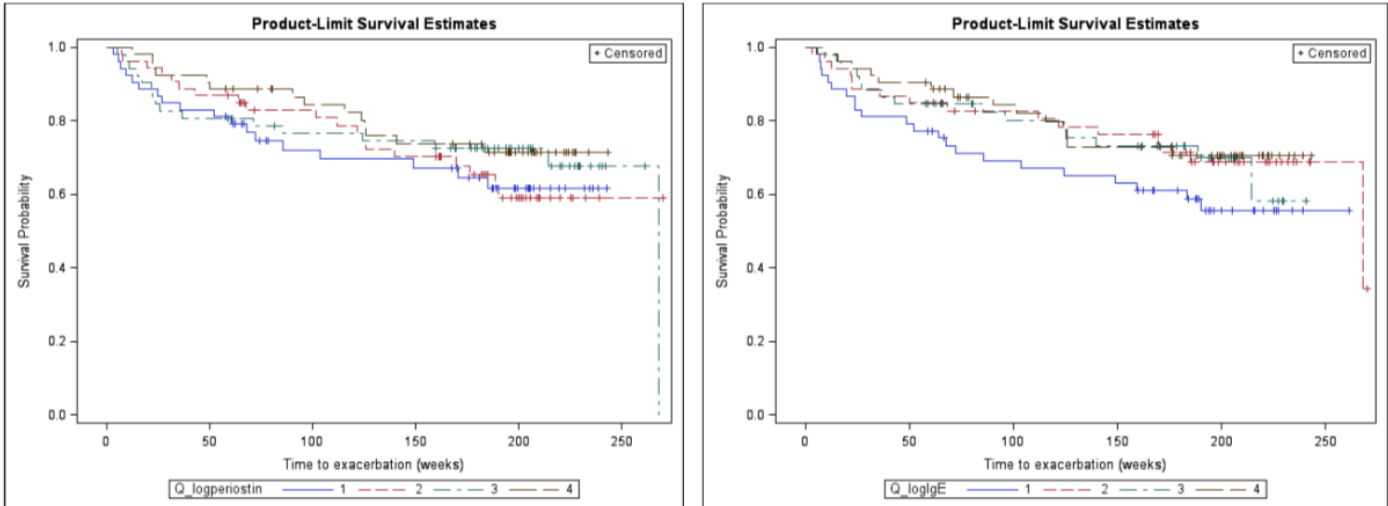

Quartile 1 is the lowest quartile and quartile 4 is the highest quartile.

ICS use at baseline

There was strong evidence of an association between ICS use at baseline and time to exacerbation with a univariate Hazard Ratio (95\% Cl) of 3.86 (1.76 to 8.48$), P<0.001$. 
Change in $\mathrm{FEV}_{1}$ over time

There was no evidence of an association between the change in $\mathrm{FEV}_{1}$ over time and baseline FeNO, serum periostin or serum IgE, (difference $(95 \% \mathrm{Cl})$ of $0.02(-0.02$ to $0.06),-0.01(-0.12$ to 0.09$)$ and $0.01(-0.01$ to 0.04$)$ in $\mathrm{FEV}_{1}$ per 0.693 increase in log FeNO, log periostin and log IgE respectively). In a multivariate model, with adjustments made for age, sex, ICS use and smoking status at baseline, there was strong evidence of an interaction between change in $\mathrm{FEV}_{1}$ between the follow up and baseline visits, and blood eosinophil count at baseline $(P<0.001)$. This was best illustrated by plotting predicted change against time, where the blood eosinophil counts of 0.1 and $0.3 \times 10^{9} / \mathrm{L}$ represented the $1^{\text {st }}$ and $3^{\text {rd }}$ quartiles, respectively (Figure 4.8). Where the baseline blood eosinophil count was 'low', the decrease in FEV 1 was more rapid with time, however 'high' baseline blood eosinophil counts were associated with an increase in $\mathrm{FEV}_{1}$ with time. The multivariate model estimated the cut-point between 'high' and 'low' to be $0.21 \times 10^{9} / \mathrm{L}$. 
Figure 4.8: Change in FEV1 with time associated with baseline eosinophil count

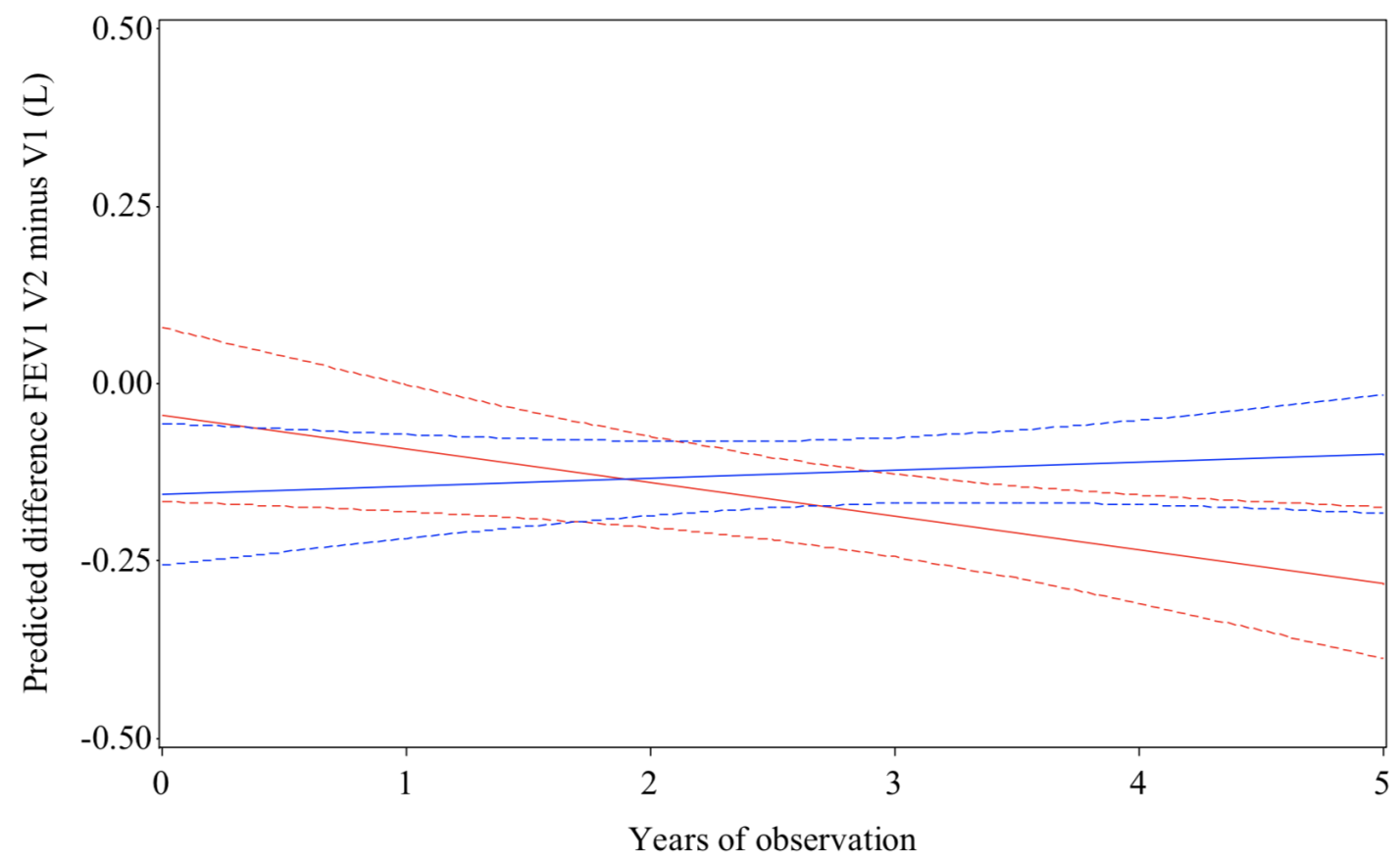

The red lines (mean prediction solid upper and lower confidence limits for the mean prediction dotted) is the predicted rate of change with time for an eosinophil count of 0.1 at baseline and the blue lines that for a baseline eosinophil count of 0.3 ; both from the model without the other co-variates. 


\subsubsection{Discussion}

This study found a statistically significant association between high baseline FeNO and serum IgE levels and a lower risk of a severe exacerbation in adults with predominantly intermittent asthma of mild to moderate severity. Those who had at least one severe exacerbation had lower baseline FeNO levels than those who did not exacerbate. The association between high baseline peripheral blood eosinophils and serum periostin levels and time to a severe exacerbation was not statistically significant. A greater decline in $\mathrm{FEV}_{1}$ was associated with a baseline peripheral blood eosinophil count of less than $0.21 \times 10^{9} / \mathrm{L}$.

These results do not confirm findings from previous randomised controlled trials of the efficacy of monoclonal antibody therapies targeting components of the Type 2 inflammatory pathways, in populations with severe asthma $52,69,202,207$ or observational cohorts with more severe persistent asthma than those in our study. ${ }^{221-223}$ This may be due to differences in the population studied, as outlined below. Although we could not identify a statistically significant association between time to severe exacerbation and blood eosinophil or serum periostin levels, the width of the confidence intervals means that we can't rule out substantial and possibly meaningful associations between time to severe exacerbation and the Type 2 measurements. For example, for blood eosinophils the lower $95 \%$ confidence limit was 0.76 which could be consistent with a strong association. 
The most important consideration in this study is confounding by ICS therapy, which is more likely to be prescribed to patients with more severe persistent disease, ${ }^{206}$ as it reduces peripheral blood eosinophil, FeNO and serum periostin levels ${ }^{213,220,224}$ and reduces the risk of severe exacerbation ${ }^{225,226}$ with clinical efficacy greater in those with raised blood or sputum eosinophils or FeNO levels. ${ }^{227,228}$ Our finding that ICS use was associated with an increased risk of severe exacerbations suggests that it was likely to be a marker of more severe disease. Furthermore, the observation that low FeNO was associated with both high inhaled fluticasone dose and shorter time to severe exacerbation may suggest that the FeNO was reduced by high dose inhaled fluticasone therapy, but that other factors associated with severe asthma may confound the association.

The other main difference is that we studied an adult population with predominantly intermittent asthma of mild to moderate severity, with only two thirds taking ICS therapy, and just less than half received concomitant LABA therapy. In contrast the randomised controlled studies of monoclonal antibody therapy which demonstrated that the Type 2 biomarkers predicted severe exacerbation risk had severe refractory asthma, despite high dose ICS therapy, with or without maintenance oral corticosteroids, and required additional controller medications. ${ }^{52,69,202,207}$

Contrasting results have been reported in observational studies such as the NHLBI Severe Asthma Program, in which there was a positive association between severe exacerbations of asthma and blood eosinophils, but not FeNO, and low serum IgE was associated with severe exacerbation risk. ${ }^{205}$ 
Our population may also explain the difference from others in our findings of an association between low blood eosinophils levels and a greater decline in lung function, and the lack of an association for either FeNO or periostin. In contrast, studies in more severe asthma populations have either shown no consistent association between accelerated decline in lung function and either high blood eosinophils, ${ }^{229} \mathrm{FeNO}^{230}$ or periostin. ${ }^{97}$

Limitations of our study include multiplicity of analyses, which may have resulted in Type 1 error but also for some of the associations assessed, the confidence intervals were quite wide. There may also have been other factors affecting an individual's risk of severe exacerbation not adjusted for in the analyses, such as baseline ICS dose, previous exacerbation history, particulate or environmental exposures, treatment adherence or socioeconomic factors, as this data was not collected at baseline. We did not investigate whether the severe exacerbations were of viral and/or allergic etiology and so differential associations based on exacerbation type could not be undertaken, additionally, this study was not designed to investigate potential mechanisms relating to the pathways associated with the Type 2 biomarkers studied, however, the positive results for FeNO and IgE and negative results for eosinophils, might indicate that the exacerbations in our population could be driven by IL-13 rather than IL-5. With respect to lung function, we did not standardise the bronchodilator withdrawal criteria before lung function testing in either the initial or the follow up study, which would have been preferable for an analysis of lung function decline. The follow up rate of $64 \%$ was lower 
than anticipated and may have resulted in selection bias in our relatively small population.

The patient's reports of severe exacerbations of asthma were corroborated with medical records where possible. All electronic records of severe exacerbations were confirmed by the investigator, regardless of whether the event was reported by the patient. Episodes where there was concordance between the patient-reported date of severe exacerbation and medical records, were confirmed by the investigator. Where the dates of severe exacerbations were not concordant with medical records, the patient-reported severe exacerbation was not counted by the investigator, unless the medical records could not be accessed such as severe exacerbations that occurred outside of New Zealand $(n=4)$ or where permission to access medical records was declined by the primary healthcare provider $(n=22)$. Given these small numbers, we do not anticipate this resulted in significant recall bias with the data.

This study comprised of two asthmatic cohorts from previous studies, which were recruited using different methodologies. The first cohort consisted of randomly selected participants from the electoral role, resulting in the inclusion of patients with mild to moderate asthma. The second cohort consisted of asthmatics on ICS and LABA therapy, to include those with more severe disease. Despite differences in recruitment methodology between the cohorts, baseline measurements for both studies were undertaken using the same guidelines and laboratory assays. In addition, all participants underwent baseline testing having been free from upper or lower 
respiratory tract infections for a minimum of three weeks, ensuring stability of disease immediately before measurements were taken.

Whereas Type 2 'high' asthma has been well characterised and described, Type 2 'low' disease is relatively under-studied. It is likely to align with the broad group of noneosinophilic asthma which is an important clinical entity comprising of multiple phenotypes with differing inflammatory profiles. ${ }^{231}$ Type 2 'low' asthmatics may have predominantly neutrophilic airway inflammation, which is the most common inflammatory phenotype in severe exacerbations. ${ }^{232}$ Significantly, Type 2 'low' asthma is relatively resistant to treatment with corticosteroid therapy, ${ }^{31,227,228,233}$ and may be the predominant mechanism in sudden-onset fatal asthma. ${ }^{234}$ Taken together, it may explain why those with low baseline blood eosinophils were more likely to have a greater decline in lung function at their follow up visit. Our findings suggest Type 2 'low' asthma may be highly prevalent among mild to moderate asthmatics in the general population which has important clinical implications, given its relative steroid insensitivity and lack of alternative treatment strategies available.

We conclude that the positive association between Type 2 biomarkers and risk of severe exacerbations in populations with severe refractory asthma may not extend to mild and moderate asthma. This study highlights the importance of Type 2 'low' asthma as a phenotype associated with an increased risk of severe exacerbations in mild to moderate asthmatics and which warrants further study. 


\section{Chapter 5: Conclusions}

\subsection{Conclusions}

Asthma is a common chronic disease with a significant healthcare burden. Despite widespread use of inhaled corticosteroid, up to $10 \%$ of asthmatics remain poorly controlled on conventional treatment. The emergence of biological therapies targeting the Type 2 inflammatory pathway has highlighted the potential role surrogate biomarkers have in identifying potential responders within a heterogeneous population. Periostin is a potential biomarker for such a use, however there was a lack of knowledge regarding its epidemiology both in non-asthmatic and asthmatic populations.

In this thesis, I have presented a series of studies regarding the epidemiology of serum periostin in non-asthmatic and asthmatic populations to establish whether or not it would be useful in a clinical setting, compared to more established Type 2 biomarkers, such as peripheral blood eosinophils, FeNO and serum IgE.

In summary, I found that serum periostin demonstrated a biphasic response to bone injury, the magnitude and duration of which was proportional to bone size. Serum levels remained elevated for up to six months after large joint replacement surgery. However, in contrast, serum periostin did not change in response to simple or surgical 
tooth extractions. Although both these studies were conducted in non-asthmatic adults, the findings are applicable to asthmatics as there is no difference in serum periostin between asthmatic and non asthmatic populations. ${ }^{100,101}$ These two studies confirm that serum periostin can vary in response to conditions other than Type 2 asthma, and that the effects on serum levels can last over six months after the event. This is of clinical relevance if serum periostin is to be used as a biomarker to identify responders to monoclonal antibody therapy in asthma.

In stable asthma, serum periostin did not vary over eight weeks, showing little intraparticipant variability. However, there was demonstrably large inter-participant variability in a relatively homogenous population with asthma. The lack of variability in serum periostin in people with stable asthma is a desirable characteristic, if it is to be used as a clinical measure of asthma control. However, the large variability in between participants suggests that there may be significant intrinsic factors which can influence serum levels. This was evident in Study 4 where serum periostin was higher in people of Chinese ethnicity than their Caucasian counterparts, irrespective of asthma status. If serum periostin is to be used in a clinical setting, race-dependent reference ranges will be required, particularly if cut-points of serum periostin determine eligibility for treatment with monoclonal antibody therapy in asthma.

Serum periostin changed in response to a severe exacerbation of asthma and its treatment and remained low for up to four weeks after the start of the exacerbation. This trend was in broad agreement with the time course of change of the other Type 2 biomarkers (peripheral blood eosinophils, FeNO and serum IgE), although the patterns 
of change between the biomarkers did not completely overlap. This suggests that they all represent different components of the Type 2 inflammatory pathway. The fact that changes of the biomarkers lasted up to four weeks post-severe exacerbation is an important consideration, particularly if these biomarkers are used to predict eligibility to monoclonal antibody therapy in asthma.

The risk of a severe exacerbation of asthma was strongly associated with low baseline FeNO levels and weakly associated with low baseline serum IgE levels. There was no association between the risk of exacerbation and peripheral blood eosinophils or serum periostin. The trend toward an inverse association between time to exacerbation and Type 2 biomarkers was a surprising finding. The most likely explanation is the high prevalence of non-Type 2 inflammation in the general population, which has been previously suggested in the literature to be between 50 and $80 \% .^{231,235}$ This is clinically important given the difference in treatment responsiveness and disease progression between Type 2 and non-Type 2 asthma.

The aim of this research was to establish the epidemiology of periostin in adults with and without asthma and to try and establish whether or not it was a good biomarker in asthma. It has previously been suggested the hallmarks of a good biomarker are to be 'minimally invasive, easily measurable and reproducible'. Periostin meets these criteria, and, from the wider literature, there is certainly a correlation between high levels of periostin and Type 2 inflammation. However, the ubiquity of a protein involved in a multitude of disease processes makes it difficult to delineate what a clinically meaningful change in serum periostin is, as demonstrated in the bone injury 
study. In addition, periostin levels are affected by ethnicity, unstable asthma and corticosteroid use, making the identification of suitable candidates for biological therapy problematic. Finally, other biomarkers, such as blood eosinophils, are just as good as periostin in identifying Type 2 asthma and come with cheaper, more readily available assays.

Before I started this body of research, periostin was emerging as potential diagnostic biomarker of asthma, identifying those with severe disease who would benefit from monoclonal antibiody therapy directed against IL-13. However, I have found periostin to be less discriminating as a surrogate biomarker: I have found that it does not distinugish between asthma and non-asthma, nor does it usefully discriminate between adults with severe and non-severe disease. This reseach has highlighted the importance of non-asthma factors which contribute toward changes in biomarkers, such as race or concurrent bone injury or use of corticosteroid. Larger observational population-based studies such as ARIETTA ${ }^{65}$, U-BIOPRED ${ }^{236}$ and ADEPT $^{237}$, looking specifically at biomarkers and disease-related health outcomes will hopefully shed some light on the increasingly complex world of surrogate biomarkers.

Asthma is such a heterogenous condition that it is unlikely that one biomarker alone will be sufficient in diagnosing or managing difficult to treat adults with asthma. I suspect that composite biomarker systems will be developed to help stratify those with asthma into categories based on severity, endotypes and help modify treatment regimens, moving ever closer to individualised medicine. 


\section{Appendix I: Participant Information}

\section{Sheet for Study One}

Participant Information Sheet

Study title: $\quad$ Serum periostin level in patients with bone fractures (PER04)

Locality: $\quad$ Medical Research Institute of Ethics committee ref.: 13/NTB/186

New Zealand

Lead Prof. Richard Beasley Contact phone number: 048050147

investigator:

You are invited to take part in a study to determine the levels of periostin, a protein in serum, over 6 months following a bone fracture, or hip or knee surgery, in a normal population of adults aged $18-75$ years. Whether or not you take part is your choice. If you don't want to take part, you don't have to give a reason, and it won't affect the care you receive. If you do want to take part now, but change your mind later, you can pull out of the study at any time.

You will not be eligible to take part in this study if any of the following applies to you:

- You have asthma, bronchitis, or COPD

- You have had wheezing or used inhalers for your breathing in the last 12 months.

- You are known to be pregnant

- You have been admitted to hospital or had surgery in the last 3 months

- You have had oral or systemic steroids in the last 3 months.

This Participant Information Sheet will help you decide if you'd like to take part. It sets out why we are doing the study, what your participation would involve, what the benefits and risks to you might be, and what would happen after the study ends. We 
will go through this information with you and answer any questions you may have. We expect this will take about 20 minutes. You may also want to talk about the study with other people, such as family, whānau, friends, or healthcare providers. Feel free to do this. The first blood sample we take (see below) will need to be within 48 hours of your fracture occurring, so please let us know if you would like to participate before this.

If you agree to take part in this study, you will be asked to sign the Consent Form on the last page of this document. You will be given a copy of both the Participant Information Sheet and the Consent Form to keep.

This document is 7 pages long, including the Consent Form. Please make sure you have read all the pages. If you require an interpreter, this will be arranged.

\section{Why are we doing the study?}

The protein periostin has been found to be present in a number of medical conditions, including asthma, early bone healing, progression of various cancers and kidney disease related to high blood pressure. It is easily measured (by a blood test) and in the future it may be used to determine the status of a person's disease, including its severity, if it is progressing or if it is no longer present. Proteins like periostin that can allow doctors to assess a disease are known as biomarkers. This study is being conducted to investigate the blood levels of periostin over 6 months following a bone fracture to provide a more complete picture of periostin production.

We aim to have a total of 102 participants involved in this study.

The Sponsor of this study is the Medical Research Institute of New Zealand. The study is being funded by Genentech Inc, a member of the Roche Group. The study has been approved by the Northern B Health and Disability Ethics Committee, reference number 13/NTB/186.

\section{What would your participation involve?}


The study is being conducted at the Medical Research Institute of New Zealand, based at Wellington Hospital. If you agree to be a part of the study you will answer a general health questionnaire and have a blood test taken after signing the consent form. You will then attend MRINZ for a further 6 visits to have a blood test taken.

\section{Visit 1 Outline}

After reading this information sheet and signing the informed consent form, the study investigator provide you with a questionnaire which you will fill out in their presence. The study investigator will then discuss your medical history and current medications so that a full picture is gained of your current health. If required we will contact your GP (with your permission) to clarify aspects of your medical history or current medications. We will then take a small blood sample to measure serum periostin level (this will be within 48 hours of your fracture occurring). This visit may take up to an hour to complete.

\section{Pre-operation Visit}

If you are undergoing hip or knee replacement surgery and agree to be part of the study (informed consent, as above), we will take a blood sample for measurement of periostin before your operation. We will then take a blood sample as detailed above at visit 1 , up to 48 hours after your surgery.

\section{Visits 2-7}

You will then be asked to attend the MRINZ at one, two, four, eight, 12 weeks and 6 months after visit 1 , to have a blood test to measure your periostin level. These visits will take no more than half an hour to complete.

\section{What are the possible benefits and risks to you of participating?}

\section{Benefits}

There will be no direct benefit to you in participating in this study, you will however be part of research which may benefit yourself and others in the future. 


\section{Blood Samples}

You may experience some discomfort during the taking of a blood sample and there is always the risk of bleeding, swelling and bruising at the site of the needle during sampling. All samples will be taken by trained staff. Around a tablespoon of blood will be taken at each study visit. In some cases we may require extra samples, for example to re-do a test that could not be analysed.

Periostin will be analysed at a central laboratory outside of New Zealand, by Genentech. This laboratory is:

Covance Central Laboratory Services

8211 Scicor Drive

Indianapolis, Indiana 46214

USA

Your blood samples will be coded with a unique subject number and your name will not be used to identify the samples. The samples will be stored for 5 years after the study, by Genentech.

You may hold beliefs about a sacred and shared value of all or any tissue samples removed. The cultural issues associated with sending your samples overseas and/or storing your tissue should be discussed with your family/whānau as appropriate. There are a range of views held by Māori around these issues; some iwi disagree with storage of samples and advise their people to consult prior to participation in research where this occurs. However it is acknowledged that individuals have the right to choose.

\section{Cost}

There will be no cost to you to take part in this study and you will be compensated $\$ 20$ for your time and travel expenses to attend your study visits.

\section{General Health Care}

Your general health care remains with your GP throughout the time you are in the study and if you experience any problems you should contact your usual health care 
provider. As part of the informed consent process we will ask for your permission to inform your GP of any unexpected clinically significant findings.

As part of the study you will be seen by study investigators, study doctors and trained staff who will conduct all the study specific assessments.

\section{What would happen if you were injured in the study?}

If you were injured as a result of treatment given as part of this study, which is unlikely, you won't be eligible for compensation from ACC. However, compensation would be available from the study's sponsor, (Medical Research Institute of New Zealand), in line with current guidelines. We can give you a copy of these guidelines if you wish. You would be able to take action through the courts if you disagreed with the amount of compensation provided.

If you have private health or life insurance, you may wish to check with your insurer that taking part in this study won't affect your cover.

\section{What are the rights of participants in the study?}

Your participation is entirely voluntary (your choice). You do not have to take part in this study, and if you choose not to take part it will in no way affect your future health care. If you do agree to take part you are free to withdraw from the study at any time, without having to give a reason.

\section{Confidentiality and Data Privacy}

If you decide to participate, the study doctor and relevant Institute staff will collect medical and personal information about you, as part of doing the study. By agreeing to take part in this research, you will allow your medical information and results to be seen by people who check that the research was done properly, for example the ethics committee and study monitor. Nothing which could personally identify you will be used in any reports on this study, or provided as part of future studies. Your personal information (for example name, sex, age and medical conditions) and other information will be identified by a study specific patient identification number (i.e. coded). The study records will be stored securely during the course of the study and 
once it is completed. After all participants have completed the study and the data has been monitored, the records will be archived for a minimum of 10 years as per New Zealand regulations. The records will then be securely destroyed.

\section{What will happen after the study ends, or if you pull out?}

Once the study has finished and the data has been analysed the results will be made available to you on your request. The sponsor may stop the study at any time. You may be asked to leave the trial, for the following reasons:

- In the Investigator's opinion it would not be in your best interest to continue in the study

- Any safety concerns

- You do not follow instructions during the study visits

Where can you go for more information about the study, or to raise concerns or complaints?

If you have any questions, concerns or complaints about the study at any stage, you can contact the study investigator:

Name: $\quad$ Dr Rachel Varughese

Phone: $\quad$ (04) 8050236 / 0278630361

E-mail: $\quad$ rachel.varughese@mrinz.ac.nz

For Maori health support, please contact:

Whānau Care Services, Cultural Care Centre, Level 2, Wellington Hospital

Phone: $\quad 0800999442$ or 048060948

Email: $\quad$ wcs@ccdhb.org.nz

If you want to talk to someone who isn't involved with the study, you can contact an independent health and disability advocate on:

Phone: $\quad 0800555050$

Fax: $\quad 08002$ SUPPORT (0800 2787 7678)

Email:_advocacy@hdc.org.nz 
You can also contact the health and disability ethics committee (HDEC) that approved this study on:

Phone: $\quad 08004$ ETHICS (0800 4384 427)

Email: $\quad$ hdecs@moh.govt.nz 


\section{Consent Form}

Study title: Serum periostin levels in patients with bone fractures (PER04)

Participant Number:

Please tick to indicate you consent to the following:

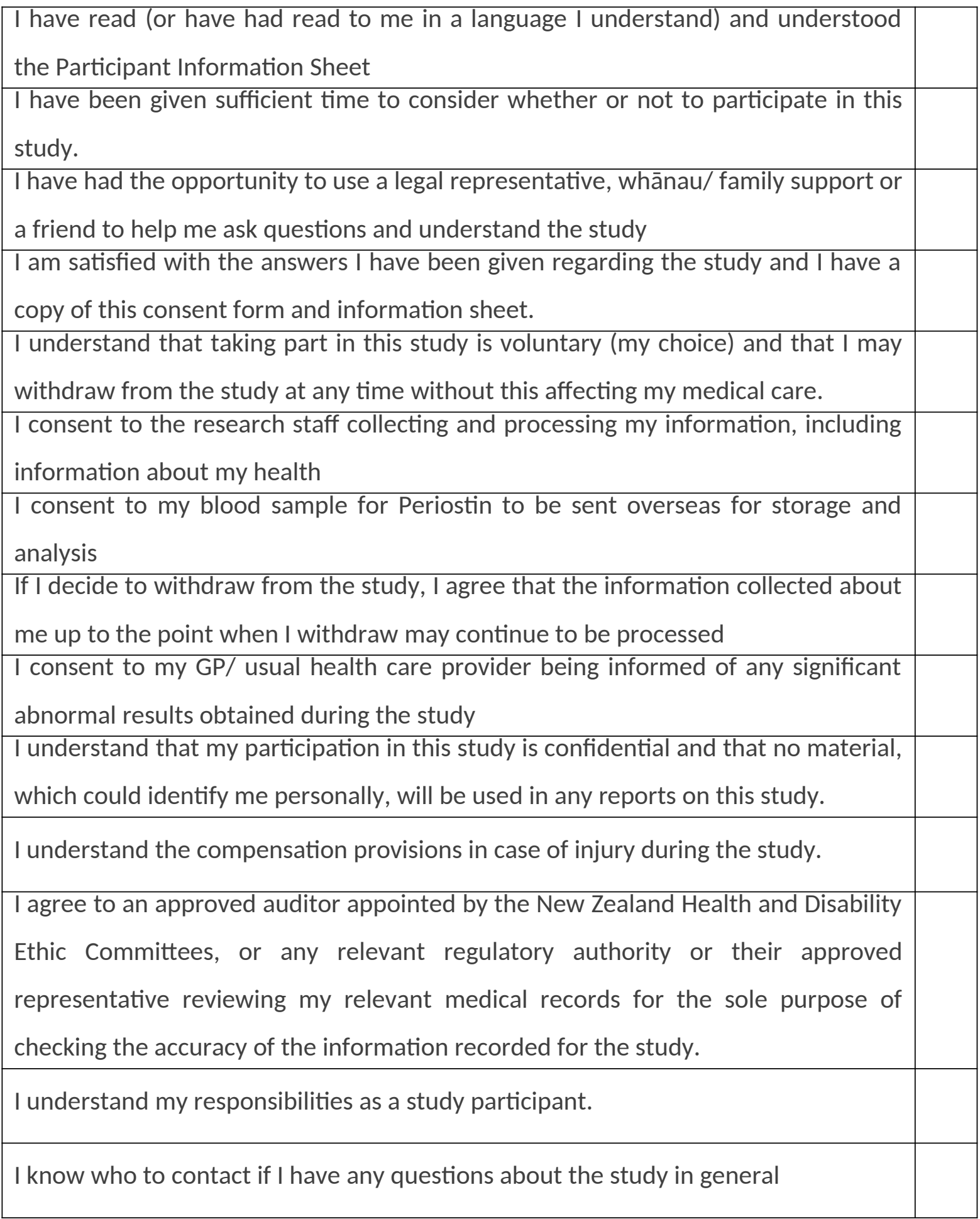




\begin{tabular}{|l|l|l|}
\hline I wish to receive a summary of the results from the study. & & \\
\hline $\begin{array}{l}\text { I give consent for study investigators to contact my GP/ usual health care } \\
\text { provider to clarify my medical history or current medications, if required }\end{array}$ & & \\
\hline $\begin{array}{l}\text { I give consent for study investigators to inform my GP/ usual health care } \\
\text { provider of my participation in this study }\end{array}$ & & \\
\hline
\end{tabular}

Declaration by participant:

I hereby consent to take part in this study

Participant name (print)

Participant signature

Date

Declaration by member of research team:

I have given a verbal explanation of the research project to the participant, and have answered the participant's questions about it.

I believe that the participant understands the study and has given informed consent to participate.

\footnotetext{
Name of person conducting informed consent discussion

(print)
}

Signature of person conducting informed consent
discussion




\section{Appendix II: Protocol for Study One}

Serum periostin level in patients with bone fractures

Protocol No.: PER04

UTN: U1111-1150-0576

ANZCTRN: ACTRN12614000151639

\section{Rationale}

Periostin, initially named osteoblast-specific factor $2,{ }^{110}$ is a matricellular protein encoded by the POSTN gene (an IL-4/ IL-13 inducible gene) thought to be an adhesion molecule because it possesses four fasciclin I domains. ${ }^{16}$ Periostin has been shown to be present in the periosteum and periodontal ligament being critical for the formation and maintenance of bone and teeth. ${ }^{238}$ Increased expression of the POSTN gene has been measured following bone fracture and has been suggested as a specific marker for preosteoblasts and periosteal callus formation in early fracture healing. ${ }^{119}$

Levels of POSTN gene expression following fracture have been measured but as serum periostin levels have not been measured following bone fractures it is difficult to determine the clinical significance of increased POSTN gene expression. This study endeavours to measure the serum periostin levels in participants with small bone and large bone fractures and in participants undergoing hip or knee joint replacements to determine if there are clinically significant changes in periostin levels following a fracture.

This study has been initiated and designed by the Medical Research Institute of New Zealand in collaboration with Genentech, Inc (a member of the Roche group). The Institute is the Sponsor of this study and undertakes Sponsor responsibilities for the purposes of ethical and any applicable regulatory requirements. Genentech are fully funding the study. 


\section{Objectives}

To estimate the magnitude and time course changes in serum periostin following a fracture.

\section{Outcomes:}

Primary outcome measure:

Serum periostin levels over a six month period following a fracture.

Secondary outcome measures:

Investigation of potential associations between serum periostin and the following:

Non-asthmatic health conditions (as captured by general health questionnaire e.g. diabetes)

\section{Study Design}

Participants:

102 participants aged 18 to 75 will be studied including:

- 34 participants with a short-bone or phalangeal fracture

- 34 participants with a long bone fracture

- 34 participants undergoing hip or knee joint replacement surgery, or open reduction internal fixation (ORIF) surgery

\section{Inclusion Criteria}

- $\quad$ Aged between 18 to 75 years

- Able to provide informed consent

- Experienced a fracture to short or long bone less than 48 hours prior, or undergoing hip or knee joint replacement (as described above).

\section{Exclusion Criteria}

- Doctor's diagnosis of Asthma, Bronchitis or COPD

- Wheezing or use of respiratory inhalers in the past 12 months

- Known pregnancy* 
- Significant co morbidities (determined at investigator discretion)

- Hospital admission within last 3 months

- Surgery within last 3 months, determined at investigator discretion (this includes all major surgery requiring general anaesthetic, dental extractions and root canal procedures. This does not include minor procedures, including but not limited to mole or wart removal, dental fillings etc).

- Systemic Corticosteroids within the last 3 months

- Any other safety concern at the investigator's discretion

* If a potential subject is aware they are currently pregnant, or actively trying to conceive, they will be excluded from the study as pregnancy can affect periostin expression. No pregnancy test will be performed on women of childbearing potential as there are no safety concerns relating to this study.

\section{Study Procedures}

Study Visits

Participants with fractures will be approached in fracture clinic or the emergency department and offered information about the study. Participants who have been treated and discharged outside of usual working hours will also be offered information about the study.

They will then complete the following:

- Written Informed Consent

- Completion of General Health Questionnaire

- Measurement of Body Mass Index (BMI)

- Laboratory samples for measurement of:

- Serum periostin level (within 48 hours of fracture, or time of surgery for those undergoing hip/knee replacement or ORIF)

Participants will then attend MRINZ at one, two, four, eight, twelve weeks and six months following the fracture to have periostin levels taken (7 visits in total). Participants 
undergoing surgery will be studied as above, except there will be an additional visit (and blood test) pre-operation. For visits $2-6$ there will be a visit window of $+/-3$ days. If a visit is delayed the calculated schedule of visit dates from baseline (visit 1) shall be maintained. Any delays greater than $+/-3$ days will be recorded as protocol deviations, and included in the final analysis.

\section{Informed Consent}

Written Informed consent will be obtained according to ethical and GCP guidelines, and will take place in either the fracture clinic or emergency department. Each participant will sign an informed consent form at the visit, prior to conducting the procedures listed above.

\section{General Health Questionnaire}

The health questionnaire will used to obtain the current health status of participants, including a history of cancer, cardiovascular disease, gastro-oesophageal reflux (GORD), rhinitis, sinusitis, and eczema (see appendix 1). As part of the assessment of general health participants will undergo height and weight measurement to obtain their BMI.

\section{Laboratory Samples}

All laboratory samples will be taken by the MRINZ study team, who are appropriately trained in phlebotomy procedures. The first sample will be taken in the fracture clinic or emergency department and the others will be taken at MRINZ.

Samples for the following tests will be sent to Genentech (or designee) to be analysed:

- Serum periostin level

- Serum periostin samples will be stored by Genentech for 5 years after the study.

\section{Safety Monitoring}

\section{Adverse Event Reporting}

For the purposes of this study, the study duration is considered to start once the informed consent form has been signed by the participant. The study period will be 
deemed to have ended when the study participant completes their last study visit and leaves MRINZ premises.

\section{Adverse Events}

An adverse event is any untoward medical occurrence in a study subject temporally associated with the testing as part of the study. An adverse event can therefore be any unfavourable and unintended sign, symptom or disease temporally associated with the testing carried out in the study. Adverse event data will be collected by investigators during the study visit and after reviewing the test results.

\section{Serious Adverse Events (SAEs)}

For the purposes of this study the following events will be considered to be SAEs:

- Death

- Life-threatening event

- Permanently disabling or incapacitating event

- Hospitalisation. Hospitalisation for the purposes of SAE reporting is defined as an admission to hospital and does not include a presentation to the Emergency Department followed by discharge without admission or an admission for elective reasons

- Any other significant medical event considered serious by the study investigator

All SAEs will be reported to the NZ HDEC and regulatory authorities as per current guidelines. SAEs and AEs will be followed up until resolution, or judged to be permanent. All SAEs will be recorded by the MRINZ (as study sponsor) within 24 hours of the study investigators becoming aware of the event.

\section{Data Safety Monitoring Committee}

Internal safety monitoring and review of safety events (adverse events and serious adverse events) will be conducted by a Medical Research Institute non-study Researcher. 


\section{Data Handling/ Statistical Methods}

\section{Study Power}

34 participants are sufficient to detect a paired difference in periostin of 0.5 of a standard deviation with $80 \%$ power. The conventional interpretation of an effect size of 0.5 of a standard deviation is of a medium effect size. For a comparison between large and small bone fractures a sample size of 68 has $90 \%$ power to detect a 0.8 standard deviation difference, a large effect size difference.

\section{Statistical analysis}

The periostin levels will be plotted against time and a non-parametric scatter plot smoother will be used to determine if there is a pattern of variation with time that might be usefully explored with parametric regression (e.g. linear, quadratic, cubic time terms). Paired t-test and mixed linear models will be used to compare the six month periostin level with measurements at the other times to formally compare the change with time.

If a highly skew distribution is found we may need to perform the analyses on the log transformed scale. 


\section{Appendix III: Participant Information}

\section{Sheet for Study Two}

Participant Information Sheet

Study title: Serum periostin levels in participants undergoing dental extractions (PER05)

Locality: $\quad$ Medical Research Institute of New

Ethics committee

13/NTB/187

Zealand

ref.:

Lead Prof. Richard Beasley

Contact phone

04805

investigator:

number:

0147

You are invited to take part in a study to determine the levels of periostin, a protein in serum, over 12 weeks following dental surgery, in a normal population of adults aged 18-75 years. Whether or not you take part is your choice. If you don't want to take part, you don't have to give a reason, and it won't affect the care you receive. If you do want to take part now, but change your mind later, you can pull out of the study at any time.

You will not be eligible to take part in this study if any of the following applies to you:

- You have asthma, bronchitis, or COPD

- You have had wheezing or used inhalers for your breathing in the last 12 months.

- You are known to be pregnant

- You have been admitted to hospital or had surgery in the last 3 months

- You have had oral or systemic steroids in the last 3 months.

This Participant Information Sheet will help you decide if you'd like to take part. It sets out why we are doing the study, what your participation would involve, what the benefits and risks to you might be, and what would happen after the study ends. We 
will go through this information with you and answer any questions you may have. We expect this will take about 20 minutes. You may also want to talk about the study with other people, such as family, whānau, friends, or healthcare providers. Feel free to do this.

If you agree to take part in this study, you will be asked to sign the Consent Form on the last page of this document. You will be given a copy of both the Participant Information Sheet and the Consent Form to keep.

This document is 7 pages long, including the Consent Form. Please make sure you have read all the pages. If you require an interpreter, this will be arranged.

\section{Why are we doing the study?}

The protein periostin has been found to be present in a number of medical conditions, including asthma, early bone healing, progression of various cancers and kidney disease related to high blood pressure. It is easily measured (by a blood test) and in the future it may be used to determine the status of a person's disease, including its severity, if it is progressing or if it is no longer present. Proteins like periostin that can allow doctors to assess a disease are known as biomarkers. This study is being conducted to investigate the blood levels of periostin over 12 weeks following dental surgery to provide a more complete picture of periostin production.

We aim to have a total of 68 participants involved in this study.

The Sponsor of this study is the Medical Research Institute of New Zealand. The study is being funded by Genentech Inc, a member of the Roche Group. The study has been approved by the Northern B Health and Disability Ethics Committee, reference number 13/NTB/187.

\section{What would your participation involve?}

The study is being conducted at the Medical Research Institute of New Zealand and dental clinics based in the Wellington Region. If you agree to be a part of the study you 
will answer a general health questionnaire and have a blood test taken after signing the consent form. We will need to take the blood test within 1 week of your tooth extraction, so please let us know if you decide to take part before this. You will then attend MRINZ, the dental clinic, or an MRINZ approved laboratory for a further 6 visits to have a blood test taken.

\section{Visit Outline}

After reading this information sheet and signing the informed consent form, the study investigator will provide you with a questionnaire which you will fill out in their presence. The study investigator will then discuss your medical history and current medications so that a full picture is gained of your current health. If required we will contact your GP (with your permission) to clarify aspects of your medical history or current medications. We will take a small blood sample to measure serum periostin level

\section{Visits 2-7}

You will then be asked to attend the MRINZ at one, two, three, four, six and 12 weeks after visit 1 , to have a blood test to measure your periostin level. This should take no more than half an hour.

\section{What are the possible benefits and risks to you of participating?}

\section{Benefits}

There will be no direct benefit to you in participating in this study, you will however be part of research which may benefit yourself and others in the future.

\section{Study Procedure Risks/ Side Effects}

Blood Samples

You may experience some discomfort during the taking of a blood sample and there is always the risk of bleeding, swelling and bruising at the site of the needle during sampling. All samples will be taken by trained staff. Around a tablespoon of blood will be taken at each study visit. In some cases we may require extra samples, for example 
to re-do a test that could not be analysed. Periostin will be analysed at a central laboratory outside of New Zealand, by Genentech. This laboratory is:

Covance Central Laboratory Services

8211 Scicor Drive

Indianapolis, Indiana 46214

USA

Your blood samples will be coded with a unique subject number and your name will not be used to identify the samples. The samples analysed locally will be destroyed by the laboratory once the results are reported. The samples sent to Genentech will be stored for 5 years after the study. You may hold beliefs about a sacred and shared value of all or any tissue samples removed. The cultural issues associated with sending your samples overseas and/or storing your tissue should be discussed with your family/whānau as appropriate. There are a range of views held by Māori around these issues; some iwi disagree with storage of samples citing whakapapa and advise their people to consult prior to participation in research where this occurs. However it is acknowledged that individuals have the right to choose.

\section{Cost}

There will be no cost to you to take part in this study and you will be compensated for your time to attend your study visits. Any reasonable travel expenses incurred travelling to Wellington Hospital from outside the Wellington Central area, will be reimbursed if the money received for study visits does not cover your costs. You will receive $\$ 70$ for the first visit and $\$ 30$ for each visit thereafter.

\section{General Health Care}

Your general health care remains with your GP throughout the time you are in the study and if you experience any problems you should contact your usual health care provider. As part of the informed consent process we will ask for your permission to inform your GP of any unexpected clinically significant findings. As part of the study you will be seen by study investigators, study doctors and trained staff who will conduct all the study specific assessments. 


\section{What would happen if you were injured in the study?}

If you were injured as a result of treatment given as part of this study, which is unlikely, you won't be eligible for compensation from ACC. However, compensation would be available from the study's sponsor, (Medical Research Institute of New Zealand), in line with current guidelines. We can give you a copy of these guidelines if you wish. You would be able to take action through the courts if you disagreed with the amount of compensation provided. If you have private health or life insurance, you may wish to check with your insurer that taking part in this study won't affect your cover.

\section{What are the rights of participants in the study?}

Your participation is entirely voluntary (your choice). You do not have to take part in this study, and if you choose not to take part it will in no way affect your future health care. If you do agree to take part you are free to withdraw from the study at any time, without having to give a reason.

\section{Confidentiality and Data Privacy}

If you decide to participate, the study doctor and relevant Institute staff will collect medical and personal information about you, as part of doing the study. By agreeing to take part in this research, you will allow your medical information and results to be seen by people who check that the research was done properly, for example the ethics committee and study monitor. Nothing which could personally identify you will be used in any reports on this study, or provided as part of future studies. Your personal information (for example name, sex, age and medical conditions) and other information will be identified by a study specific patient identification number (i.e. coded). The study records will be stored securely during the course of the study and once it is completed. After all participants have completed the study and the data has been monitored, the records will be archived for a minimum of 10 years as per New Zealand regulations. The records will then be securely destroyed. 
What will happen after the study ends, or if you pull out?

Once the study has finished and the data has been analysed the results will be made available to you on your request. The sponsor may stop the study at any time. You may be asked to leave the trial, for the following reasons:

- In the Investigator's opinion it would not be in your best interest to continue in the study

- Any safety concerns

- You do not follow instructions during the study visits

Where can you go for more information about the study, or to raise concerns or complaints?

If you have any questions, concerns or complaints about the study at any stage, you can contact the study investigator:

Name: $\quad$ Ruth Semprini

Phone: $\quad 8050232$

E-mail: $\quad$ ruth.semprini@mrinz.ac.nz

For Maori health support, please contact:

Whānau Care Services, Cultural Care Centre, Level 2, Wellington Hospital

Phone: $\quad 0800999442$ or 048060948

Email: $\quad$ wcs@ccdhb.org.nz

If you want to talk to someone who isn't involved with the study, you can contact an independent health and disability advocate on:

Phone: $\quad 0800555050$

Fax: $\quad 08002$ SUPPORT (0800 2787 7678)

Email: $\quad$ advocacy@hdc.org.nz

You can also contact the health and disability ethics committee (HDEC) that approved this study on:

Phone: $\quad 08004$ ETHICS (0800 4384 427)

Email: $\quad$ hdecs@moh.govt.nz 


\section{Consent Form}

Study title: Serum periostin levels in participants undergoing major dental surgery (PER05)

Participant Number:

Please tick to indicate you consent to the following:

\begin{tabular}{|c|}
\hline $\begin{array}{l}\text { I have read (or have had read to me in a language I understand) and understood the } \\
\text { Participant Information Sheet }\end{array}$ \\
\hline I have been given sufficient time to consider whether or not to participate in this study \\
\hline $\begin{array}{l}\text { I have had the opportunity to use a legal representative, whānau/ family support or a } \\
\text { friend to help me ask questions and understand the study }\end{array}$ \\
\hline $\begin{array}{l}\text { I am satisfied with the answers I have been given regarding the study and I have a copy } \\
\text { of this consent form and information sheet }\end{array}$ \\
\hline $\begin{array}{l}\text { I understand that taking part in this study is voluntary (my choice) and that I may } \\
\text { withdraw from the study at any time without this affecting my medical care. }\end{array}$ \\
\hline $\begin{array}{l}\text { I consent to the research staff collecting and processing my information, including } \\
\text { information about my health }\end{array}$ \\
\hline I consent to my blood sample for Periostin to be sent overseas for storage and analysis \\
\hline $\begin{array}{l}\text { If I decide to withdraw from the study, I agree that the information collected about me } \\
\text { up to the point when I withdraw may continue to be processed }\end{array}$ \\
\hline $\begin{array}{l}\text { I consent to my GP or current health care provider being informed of any significant } \\
\text { abnormal results obtained during the study }\end{array}$ \\
\hline $\begin{array}{l}\text { I understand that my participation in this study is confidential and that no material, } \\
\text { which could identify me personally, will be used in any reports on this study }\end{array}$ \\
\hline I understand the compensation provisions in case of injury during the study \\
\hline $\begin{array}{l}\text { I agree to an approved auditor appointed by the New Zealand Health and Disability } \\
\text { Ethic Committees, or any relevant regulatory authority or their approved } \\
\text { representative reviewing my relevant medical records for the sole purpose of checking } \\
\text { the accuracy of the information recorded for the study }\end{array}$ \\
\hline I understand my responsibilities as a study participant \\
\hline I know n \\
\hline
\end{tabular}




\begin{tabular}{|l|l|l|}
\hline \multicolumn{1}{|c|}{ Yes } & No \\
\hline I wish to receive a summary of the results from the study & & \\
\hline $\begin{array}{l}\text { I give consent for study investigators to contact my GP/ usual health care provider } \\
\text { to clarify my medical history or current medications, if required }\end{array}$ & & \\
\hline $\begin{array}{l}\text { I give consent for study investigators to inform my GP/ usual health care provider } \\
\text { of my participation in this study }\end{array}$ & & \\
\hline
\end{tabular}

\section{Declaration by participant:}

I hereby consent to take part in this study

Participant name (print)

Participant signature

Date

\section{Declaration by member of research team:}

I have given a verbal explanation of the research project to the participant, and have answered the participant's questions about it. I believe that the participant understands the study and has given informed consent to participate.

\section{Name of person conducting informed consent discussion (print)}

Signature of person conducting informed consent
discussion




\section{Appendix IV: Protocol for Study Two}

Serum periostin levels in participants undergoing major dental surgery

Protocol No.: PER05

UTN: U1111-1150-3950

ANZCTRN: ACTRN12614000152628

\section{Rationale}

Periostin, initially named osteoblast-specific factor $2,{ }^{110}$ is a matricellular protein encoded by the POSTN gene (an IL-4/ IL-13 inducible gene) thought to be an adhesion molecule because it possesses four fasciclin I domains. ${ }^{16}$ Periostin has been shown to be present in the periosteum and periodontal ligament being critical for the formation and maintenance of bone and teeth. ${ }^{238}$

Periostin is associated with type I collagen in the periodontal ligament where it regulates fibrillogenesis and the biomechanical properties of connective tissue around the tooth. ${ }^{110}$ Increased expression of the POSTN gene has been measured in the periodontal ligament on occlusal loading, investigators concluding that periostin is therefore essential for the integrity and function of the ligament. ${ }^{110}$

The serum levels of periostin following dental surgery have not yet been investigated. This study of the magnitude and longitudinal changes in serum periostin levels in participants undergoing dental surgery endeavours to determine the clinical significance of periostin following dental surgery. Results from this study will provide a clearer picture of periostin production and further the development of periostin as a serum biomarker for asthma.

This study has been initiated and designed by the Medical Research Institute of New Zealand in collaboration with Genentech, Inc (a member of the Roche group). The Institute is the Sponsor of this study and undertakes Sponsor responsibilities for the 
purposes of ethical and any applicable regulatory requirements. Genentech are fully funding the study.

\section{Objectives}

To estimate the magnitude and time course changes in serum periostin following dental extractions, with or without surgery.

\section{Outcomes:}

Primary outcome measure:

Serum periostin levels over a 12 week period following dental extraction

Secondary outcome measures:

Investigation of potential associations between serum periostin and the following: Non-asthmatic health conditions (as captured by general health questionnaire e.g. diabetes)

\section{Study Design}

Participants:

Participants attending a dental clinic, for dental extraction/surgery.

68 participants aged 18 to 75 will be studied including participants undergoing a simple and/or surgical tooth extractions.

For the purposes of this study a 'surgical' tooth extraction requires cutting into bone to remove the tooth. Simple tooth extractions do not involve the bone. If the participant is not sure of what type of extraction they are scheduled to have, clarification should be sought by the study investigator from the Dentist performing the extraction.

\section{Inclusion Criteria}

- $\quad$ Aged between 18 to 75 years 
- Able to provide informed consent

- Scheduled for dental extraction \pm surgery

\section{Exclusion Criteria}

- Doctor's diagnosis of Asthma, Bronchitis or COPD

- Wheezing or use of respiratory inhalers in the past 12 months

- Known pregnancy*

- Significant co morbidities (determined at investigator discretion)

- Hospital admission within last 3 months

- Surgery within last 3 months, determined at investigator discretion (this includes all major surgery requiring general anaesthetic, dental extractions and root canal procedures. This does not include minor procedures, including but not limited to mole or wart removal, dental fillings etc).

- Oral or Systemic Corticosteroids within the last 3 months

- Any other safety concern at the investigator's discretion

* If a potential subject is aware they are currently pregnant, or actively trying to conceive, they will be excluded from the study.

\section{Study Procedures}

\section{Study Visits}

Participants will be identified through dental surgery lists at designated dental clinics. Participants will be offered information on the study and if they consent they will complete a general health questionnaire in the presence of a study investigator and then have a pre-surgery/extraction blood sample taken. This sample will be taken up to one week prior to the simple tooth extraction, or surgery. Participants will be approached at the dental clinic and the following will be completed:

- Written Informed Consent

- Completion of General Health Questionnaire

- Measurement of Body Mass Index (BMI)\#

- Laboratory samples for measurement of: 
- Serum periostin level

"Should it not be possible to complete the General Health Questionnaire and BMI at Visit 1 (e.g. due to participant time constraints), then this may be completed at Visit 2 (week one). Smoking and exercise history on the General Health Questionnaire will be captured up to the date of Visit 1 only. Participants will then attend MRINZ, an MRINZ designated laboratory, or the dental clinic as convenient, at one, two, three, four, six and 12 weeks following dental extraction, to have periostin levels taken (7 visits in total). If necessary, blood samples may be taken at the participant's home, in line with MRINZ health and safety policy. For visits $2-7$ there will be a visit window of $+/-3$ days. If a visit is delayed the calculated schedule of visit dates from baseline (visit 1) shall be maintained. Any delays greater than +/- 3 days will be recorded as protocol deviations, and included in the final analysis.

Visit Schedule:

\begin{tabular}{|l|l|}
\hline Visit Number & Scheduled time \\
\hline Visit 1 & Up to 1 week prior to extraction \\
\hline Visit 2 & 1 week POST extraction (+/- 3 Days) \\
\hline Visit 3 & 2 weeks POST extraction (+/- 3 days) \\
\hline Visit 4 & 3 weeks POST extraction (+/- 3 days) \\
\hline Visit 5 & 4 weeks POST extraction (+/- 3 days) \\
\hline Visit 6 & 6 weeks POST extraction (+/- 3 days) \\
\hline Visit 7 & 12 weeks POST extraction (+/- 3 days) \\
\hline
\end{tabular}

\section{Informed Consent}

Written informed consent will be obtained according to ethical and GCP guidelines, and will take place at the dental clinic. Each participant will sign an informed consent form at the visit, prior to conducting the procedures listed above.

\section{General Health Questionnaire}

The health questionnaire will be used to obtain the current health status of participants, including a history of cancer, cardiovascular disease, gastro-oesophageal reflux (GORD), rhinitis, sinusitis, and eczema (see appendix 1). As part of the assessment of general health participants will undergo height and weight measurement to obtain their BMI. 


\section{Laboratory Samples}

All laboratory samples will be taken by staff appropriately trained in phlebotomy procedures. Samples may be taken at the dental clinic, the MRINZ, or a laboratory site approved by the MRINZ, or the participant's home, as outlined in study visits.

Samples for the following tests will be sent to Genentech (or designee) to be analysed:

- Serum periostin level

- Safety Monitoring

\section{Adverse Event Reporting}

For the purposes of this study, the study duration is considered to start once the informed consent form has been signed by the participant. The study period will be deemed to have ended when the study participant completes their final study visit and leaves MRINZ premises.

\section{Adverse Events}

An adverse event is any untoward medical occurrence in a study subject temporally associated with the testing as part of the study. An adverse event can therefore be any unfavourable and unintended sign, symptom or disease temporally associated with the testing carried out in the study. Adverse event data will be collected by investigators during the study visit and after reviewing the test results.

\section{Serious Adverse Events (SAEs)}

For the purposes of this study the following events will be considered to be SAEs:

- Death

- Life-threatening event

- Permanently disabling or incapacitating event

- Hospitalisation. Hospitalisation for the purposes of SAE reporting is defined as an admission to hospital and does not include a presentation to the Emergency Department followed by discharge without admission or an admission for elective reasons 
- Any other significant medical event considered serious by the study investigator

All SAEs will be reported to the NZ HDEC and regulatory authorities as per current guidelines. SAEs and AEs will be followed up until resolution, or judged to be permanent. All SAEs will be recorded by the MRINZ (as study sponsor) within 24 hours of the study investigators becoming aware of the event.

\section{Data Safety Monitoring Committee}

Internal safety monitoring and review of safety events (adverse events and serious adverse events) will be conducted by a Medical Research Institute non-study Researcher.

\section{Data Handling/ Statistical Methods}

Study Power

While the final balance of participants between simple and surgical extractions will not be known until the end of recruitment, 34 participants in each group are sufficient to detect a paired difference in periostin of 0.5 of a standard deviation with $80 \%$ power. The conventional interpretation of an effect size of 0.5 of a standard deviation is of a medium effect size. For a comparison between large and small dental procedures a sample size of 68 has a $90 \%$ power to detect a 0.8 standard deviation difference, a large effect size difference.

\section{Statistical analysis}

The periostin levels will be plotted against time and a non-parametric scatter plot smoother will be used to determine if there is a pattern of variation with time that might be usefully explored with parametric regression (e.g. linear, quadratic, cubic time terms). Paired t-test and mixed linear models will be used to compare the 12 week periostin level with measurements at the other times to formally compare the change with time. If a highly skew distribution is found we may need to perform the analyses on the log transformed scale. 


\title{
Appendix V: Participant Information
}

\section{Sheet for Study Three}

\author{
Study title: Periostin levels in those of Chinese descent (PER07) \\ Locality: $\quad$ Medical Research Institute Ethics \\ 13/NTB/190 \\ of New Zealand c committee ref.: \\ Lead Prof. Richard Beasley $\quad$ Contact phone 048050147 \\ investigator: number:
}

You are invited to take part in a study to determine the levels of periostin, a protein in serum, in an asthmatic population of adults aged 18-75 years, of Chinese descent. Whether or not you take part is your choice. If you don't want to take part, you don't have to give a reason, and it won't affect the care you receive. If you do want to take part now, but change your mind later, you can pull out of the study at any time.

You will not be eligible to take part in this study if any of the following applies to you:

- You are pregnant

- You have been admitted to hospital or had surgery in the last 3 months

- You have had a bone fracture in the last 3 months

- Current smoker, or a former smoker ( >10 pack-years)

- You currently have (or within the 3 weeks prior to visit) a respiratory tract infection

This Participant Information Sheet will help you decide if you'd like to take part. It sets out why we are doing the study, what your participation would involve, what the benefits and risks to you might be, and what would happen after the study ends. We will go through this information with you and answer any questions you may have. We expect this will take about 20 minutes. You may also want to talk about the study with other people, such as family, whānau, friends, or healthcare providers. Feel free to do this. If you agree to take part in this study, you will be asked to sign the Consent Form 
on the last page of this document. You will be given a copy of both the Participant Information Sheet and the Consent Form to keep.

This document is 8 pages long, including the Consent Form. Please make sure you have read all the pages. If you require an interpreter, this will be arranged.

\section{Why are we doing the study?}

The protein periostin has been found to be present in a number of medical conditions, including asthma, early bone healing, progression of various cancers and kidney disease related to high blood pressure. It is easily measured (by a blood test) and in the future it may be used to determine the status of a person's disease, including its severity, if it is progressing or if it is no longer present. Proteins like periostin that can allow doctors to assess a disease are known as biomarkers.

This study is being conducted to determine the normal population reference range for periostin in adults of Chinese descent as there has been indication from previous studies that periostin levels may differ in those of Chinese heritage. This study is also looking to see if there is a difference in periostin levels in asthmatic and non-asthmatic participants of Chinese descent. We aim to recruit a total of 68 participants in this part of the study; 34 participants with mild asthma and another 34 participants with moderate/severe asthma. For this study, participants with mild and moderate/severe asthma is defined as participants treated with short-acting $\beta$-agonist (SABA) only and participants treated with SABA and inhaled corticosteroid therapy (ICS) respectively.

The Sponsor of this study is the Medical Research Institute of New Zealand. The study is being funded by Genentech Inc, a member of the Roche Group. The study has been approved by the Northern B Health and Disability Ethics Committee, reference number 13/NTB/190.

\section{What would your participation involve?}

The study is being conducted at the Medical Research Institute of New Zealand (MRINZ). If you agree to be a part of the study you will need to attend the research facility for one visit lasting approximately 100 minutes (slightly more than an hour and 
a half). The study visit will involve breathing exercises (including spirometry tests), and a blood test. You will also be asked to fill out a general health questionnaire and discuss your medical history and current medications. You will be asked to bring in your current medications and to withhold the following prior to the breathing tests:

- Withhold caffeine for 6 hours prior to your appointment.

- Withhold food for 1 hour prior to your appointment

\section{Visit Outline}

After reading this information sheet and signing the informed consent form, the study investigator provide you with two respiratory health questionnaires which you will fill out in their presence. A third questionnaire about your general health will be administered by the investigator. The study investigator will then discuss your current medications so that a full picture is gained of your current health. If required, we will contact your GP (with your permission) to clarify aspects of your medical history or current medications. You will also be asked to complete a genogram for you, your parents and your grandparents, to document ethnicity and country of birth.

We will measure nitric oxide (a gas that you normally breathe out) by asking you to breathe into a tube. To check the health and function of your lungs you will be asked to do some breathing exercises. You will then follow the instructions given to you by the investigator, for a few minutes. This test will be done so that 3 of your best measurements are recorded. This may take up to 8 measurements (or more if you agree) in total, so that we can record the best results.

We will take a small blood sample, to measure your full blood count, urea, electrolytes and creatinine as well as serum IgE, serum periostin level and other immune mediated biomarkers.

\section{Travel}

If you live in a region of New Zealand other than Auckland or Wellington, we will arrange for you to attend your study visit at MRINZ in Wellington and pay for all associated travel costs. This might involve air travel and an extra time commitment on your part besides the time required for the study visit itself. 


\section{What are the possible benefits and risks to you of participating? Benefits}

There will be no direct benefit to you in participating in this study, you will however be part of research which may benefit yourself and others in the future.

\section{Study Procedure Risks/ Side Effects}

\section{Blood Samples}

You may experience some discomfort during the taking of a blood sample and there is always the risk of bleeding, swelling and bruising at the site of the needle during sampling. All samples will be taken by trained staff. Around 2 tablespoons of blood will be taken during the study. In some cases we may require extra samples, for example to re-do a test that could not be analysed. A local laboratory will analyse your full blood count, urea, electrolytes and creatinine as well as serum IgE.

Periostin will be analysed at a central laboratory outside of New Zealand, by Genentech. This laboratory is:

Covance Central Laboratory Services

8211 Scicor Drive

Indianapolis, Indiana 46214

USA

Your blood samples will be coded with a unique subject number and your name will not be used to identify the samples. The samples analysed locally will be destroyed by the laboratory once the results are reported. The samples sent to Genentech will be stored for 5 years after the study. You may hold beliefs about a sacred and shared value of all or any tissue samples removed. The cultural issues associated with sending your samples overseas and/or storing your tissue and/or undertaking genetic analysis on them should be discussed with your family/whānau as appropriate. There are a range of views held by Māori around these issues; some iwi disagree with storage of samples and genetic testing citing whakapapa and advise their people to consult prior to participation in research where this occurs. However, it is acknowledged that individuals have the right to choose. 


\section{Breathing Exercises}

You may feel some discomfort during or after performing the breathing exercises, however this will be transient and you will be monitored constantly throughout the tests by trained technicians/ study investigators. You will be seated at all times for the tests.

Cost

There will be no cost to you to take part in this study and you will be compensated $\$ 50$ for your time and travel expenses (if you live in Wellington) to attend your study visit.

\section{General Health Care}

Your general health care remains with your GP throughout the time you are in the study and if you experience any problems you should contact your usual health care provider. As part of the informed consent process we will ask for your permission to inform your GP of any unexpected clinically significant findings.

As part of the study you will be seen by study investigators, study doctors and trained staff who will conduct all the study specific assessments.

\section{What would happen if you were injured in the study?}

If you were injured as a result of treatment given as part of this study, which is unlikely, you won't be eligible for compensation from ACC. However, compensation would be available from the study's sponsor (Medical Research Institute of New Zealand), in line with current guidelines. We can give you a copy of these guidelines if you wish. You would be able to take action through the courts if you disagreed with the amount of compensation provided. If you have private health or life insurance, you may wish to check with your insurer that taking part in this study won't affect your cover.

\section{What are the rights of participants in the study?}

Your participation is entirely voluntary (your choice). You do not have to take part in this study, and if you choose not to take part it will in no way affect your future health 
care. If you do agree to take part you are free to withdraw from the study at any time, without having to give a reason.

\section{Confidentiality and Data Privacy}

If you decide to participate, the study doctor and relevant Institute staff will collect medical and personal information about you, as part of doing the study.

By agreeing to take part in this research, you will allow your medical information and results to be seen by people who check that the research was done properly, for example the ethics committee and study monitor. Nothing which could personally identify you will be used in any reports on this study, or provided as part of future studies. Your personal information (for example name, sex, age and medical conditions) and other information will be identified by a study specific patient identification number (i.e. coded). The study records will be stored securely during the course of the study and once it is completed. After all participants have completed the study and the data has been monitored, the records will be archived for a minimum of 10 years as per New Zealand regulations. The records will then be securely destroyed.

\section{What will happen after the study ends, or if you pull out?}

Once the study has finished and the data analysed, the results will be made available to you on your request. You may also request a copy of your individual lung function results.

The sponsor may stop the study at any time. You may be asked to leave the trial, for the following reasons:

- In the Investigator's opinion it would not be in your best interest to continue in the study

- Any safety concerns

- You do not follow instructions during the study visit

Where can you go for more information about the study, or to raise concerns or complaints? 
If you have any questions, concerns or complaints about the study at any stage, you can contact the study investigator:

$\begin{array}{ll}\text { Name: } & \text { Evan Tan } \\ \text { Phone: } & 04 \text { 805-0249 } \\ \text { E-mail: } & \text { evan.tan@mrinz.ac.nz }\end{array}$

For Maori health support, please contact:

Whānau Care Services, Cultural Care Centre, Level 2, Wellington Hospital

Phone: $\quad 0800999442$ or 048060948

Email: $\quad$ wcs@ccdhb.org.nz

If you want to talk to someone who isn't involved with the study, you can contact an independent health and disability advocate on:

Phone: $\quad 0800555050$

Fax: $\quad 08002$ SUPPORT (0800 2787 7678)

Email: $\quad$ advocacy@hdc.org.nz

You can also contact the health and disability ethics committee (HDEC) that approved this study on:

Phone: $\quad 08004$ ETHICS (0800 4384 427)

Email: $\quad \underline{\text { hdecs@moh.govt.nz }}$ 


\section{Consent Form}

Study title: Periostin levels in those of Chinese descent (PER07)

Participant Number:

\section{Please tick to indicate you consent to the following:}

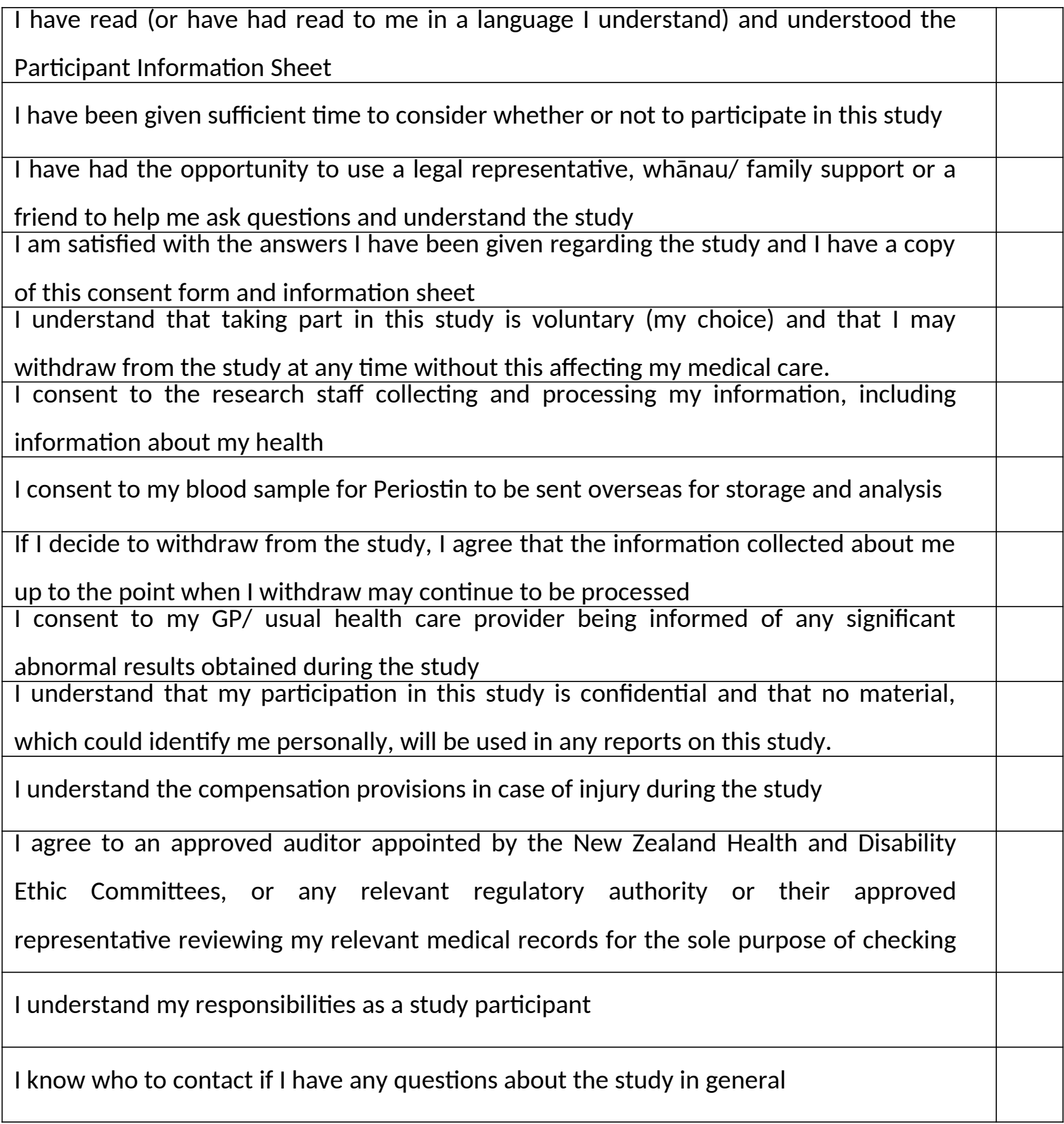




\begin{tabular}{|l|c|}
\hline & \\
\hline I wish to receive a summary of the results from the study. & \\
\hline \multicolumn{1}{|c|}{ Yes } & \multicolumn{1}{|c|}{ No } \\
\hline $\begin{array}{l}\text { I give consent for study investigators to contact my GP/ usual health care } \\
\text { provider to clarify my medical history or current medications, if required }\end{array}$ & \\
\hline
\end{tabular}

Declaration by participant:

I hereby consent to take part in this study

Participant name (print)

Participant signature

Date

\section{Declaration by member of research team:}

I have given a verbal explanation of the research project to the participant, and have answered the participant's questions about it.

I believe that the participant understands the study and has given informed consent to participate.

Name of person conducting informed consent discussion (print)

Signature of person conducting informed consent Date discussion 
Declaration by member of research team:

I have given a verbal explanation of the research project to the participant, and have answered the participant's questions about it.

I believe that the participant understands the study and has given informed consent to participate.

Name of person conducting informed consent discussion

(print)

Signature of person conducting informed consent Date

discussion 


\section{Appendix VI: Protocol for Study Three}

Protocol

Periostin levels in those of Chinese descent

Protocol No.: PER07

UTN: U1111-1150-5103

ANZCTRN: ACTRN12614000122651

\section{Rationale}

Periostin is a matricellular protein encoded by the POSTN gene (an IL-4/ IL-13 inducible gene) thought to be an adhesion molecule because it possesses four fasciclin I domains. ${ }^{16}$ Periostin has been shown to be secreted in the extracellular matrix from the basolateral surface of bronchial epithelial cells after stimulation with IL-13. ${ }^{98}$

Periostin has been studied predominantly in patients with asthma, expression levels of the POSTN gene corresponding to reticular basement membrane thickness an indicator of subepithelial fibrosis. ${ }^{98}$ Periostin is thought to enhance fibrosis in the airways by binding to other extracellular proteins collagen, fibronectin and tenascin C. ${ }^{239}$ Periostin levels correlate with eosinophilic airway inflammation and may also have a role in TH2 driven inflammation as seen in nasal polyps, eosinophilic oesophagitis, allergic rhinitis and atopic dermatitis.

Increased expression of the POSTN gene has been measured following bone fracture and has been suggested as a specific marker for preosteoblasts and periosteal callus formation in early fracture healing. ${ }^{119}$ Periostin has also been shown to be present in the periosteum and periodontal ligament being critical for the formation and maintenance of bone and teeth. ${ }^{238}$ 
Periostin has also been shown to have a role in the repair of both skin and cardiac tissue following injury ${ }^{240}$ and to be expressed by various types of cancer as well as being involved in the formation of distant metastases. ${ }^{238}$

Periostin levels have been measured in a number of tissues and disease states and recent results from studies measuring serum periostin levels have indicated that there may be a difference in serum periostin levels in patients of Chinese descent. This study endeavours to provide serum periostin levels in a $1^{\text {st }}$ and $2^{\text {nd }}$ generation healthy Chinese population which will then be compared to levels found in healthy Caucasians. Results from this study will be used to determine whether a serum periostin reference range is required for the Chinese population.

This study has been initiated and designed by the Medical Research Institute of New Zealand (MRINZ) in collaboration with Genentech, Inc (a member of the Roche group). The Institute is the Sponsor of this study and undertakes Sponsor responsibilities for the purposes of ethical and any applicable regulatory requirements. Genentech are fully funding the study.

\section{Objectives}

- To determine a serum periostin reference range for an adult Chinese population.

- To compare serum periostin levels in Caucasian and Chinese populations.

- To determine if country of birth influences periostin levels.

- To determine if there is a difference in periostin levels between Chinese adults with and without asthma.

\section{Outcomes:}

\section{Primary outcome measure:}

- Reference range (mean, median and 90\% confidence intervals) of periostin levels in a non-asthmatic adult Chinese population. 


\section{Main secondary outcome measures:}

- Comparison of the periostin levels in non-asthmatic Caucasian and Chinese populations.

- Comparison of the periostin levels in non-asthmatic and asthmatic Chinese adults.

- Determine the effects of country of birth (New Zealand and Australia vs Asia) on periostin levels.

- Comparison of the FeNO, blood eosinophil and serum IgE levels in nonasthmatic Caucasian and Chinese populations.

- Comparison of periostin levels between mild and moderate/severe asthmatics of Chinese descent.

\section{Exploratory outcome measures (RCR only):}

- Other immune mediated biomarkers

- DNA and RNA- based biomarker analyses

\section{Study Design}

\section{Participants:}

The study will consist of two groups of adult Chinese participants making up a total of 188 adults aged 18 to 75 years, and 364 Caucasian adults (already investigated).

Group 1: consists of 120 non-asthmatic participants, with at least 20 participants (10 male and 10 female) recruited to each of the following four age bands; $18-30,31-45$, 46-60 and 61-75 years.

A total of 364 Caucasian adults aged 18 to 75 years, studied in 'Periostin Study 1" (reference ranges for serum periostin in a population without asthma or COPD), that meet the smoking criteria of this study (Periostin Study 7), will be included as the Caucasian reference population. 
Group 2: consists of 68 participants with a doctor's diagnosis of asthma, aged 18 to 75 years. More specifically, the participants will include:

- 34 participants with mild asthma; participants treated with short-acting $\beta$ agonist (SABA) only

- 34 participants with moderate/severe asthma; participants treated with at least inhaled corticosteroid (ICS) therapy.

\section{Inclusion Criteria}

- Between 18 to 75 years of age

- Able to provide written informed consent

- Born in New Zealand, Australia, China, Hong Kong, Taiwan, Singapore or other countries in Asia

- Of Chinese descent, more specifically:

- Both parents that self-identify as ethnic Chinese

- Residing in New Zealand for more than one year at Visit 1

- For Group 1 (non-asthmatics ${ }^{\#)}$, at least 10 male and 10 female participants will be recruited to each of the following four age bands; 18-30, 31-45, 46-60 and 61-75 years.

- For Group 2 (asthmatics), participants will have a current diagnosis of asthma. 34 will have mild asthma (treated with a SABA) and 34 will have moderate/severe asthma (treated with an ICS).

\#Potential subjects with a historic diagnosis of asthma or bronchitis will be deemed eligible for the study. This is defined as the subject having had no symptoms of asthma or bronchitis, and has neither taken nor had prescribed any medications for the treatment of those conditions, within 10 years prior to the study visit. A prior diagnosis of COPD at any time will result in exclusion of a potential subject. This is consistent with 'Periostin Study 1' and will enable comparison between the two groups.

\section{Exclusion Criteria}

Group 1

- Doctor's diagnosis of Asthma, Chronic Bronchitis or COPD 
- Wheezing or use of respiratory inhalers in the past 12 months

- Systemic Corticosteroids within the last three months

Group 1 and 2

- Current smoker, or former smoker with a smoking history of $>10$ pack-years.

- Known pregnancy*

- First six weeks post-partum

- Significant co morbidities (determined at investigator discretion)

- Hospital admission within last three months

- Surgery within last three months, determined at investigator discretion (this includes all major surgery requiring general anaesthetic, dental extractions and root canal procedures. This does not include minor procedures, including but not limited to mole or wart removal, dental fillings etc).

- Bone fracture within the last three months

- Active (current, or within the three weeks prior to the visit) upper or lower respiratory tract infection

- Any other safety concern at the investigator's discretion

*If a potential subject is aware they are currently pregnant, or actively trying to conceive, they will be excluded from the study as pregnancy can affect FeNO, eosinophil and spirometry results. No pregnancy test will be performed on women of childbearing potential as there are no safety concerns relating to this study.

Participants may be re-screened at a suitable time should it become apparent they cannot fulfil eligibility criteria at their initial visit (for example they are experiencing a respiratory infection).

\section{Study Procedures}

\section{Study Visits}

Participants will present at the research facility (MRINZ or Optimal Clinical Trials) for a single visit, with the following procedures being completed:

- Written Informed Consent 
- Completion of General Health Questionnaire

- Completion of Genogram (to document ethnicity and country of birth of participant, participant's parents and participant's grandparents)

- Measurement of Body Mass Index (BMI)

- Spirometry assessment (Measurement of FEV ${ }_{1}$ and FVC)

- Measurement of FeNO

In participants with Asthma, two respiratory health questionnaires will be administered to determine current asthma control (ACQ-5 and AQLQ-S). Self-reported date of last use of oral steroids and exacerbation of asthma will be documented.

Laboratory samples for measurement of:

- Full blood count (including differential white cell count)

- Creatinine, urea and electrolytes

- Serum IgE

- Serum periostin level

- Serum, DNA and RNA based biomarker analyses will also be conducted on samples collected for the Roche Clinical Repository (RCR). These samples will only be collected from participants who provide consent on the RCR informed consent form which is a separate consent to the main study (see Optional samples for RCR).

\section{Informed Consent}

Written informed consent will be obtained according to ethical and Good Clinical Practice (GCP) guidelines, and will take place at the research facility. Each participant will sign an informed consent form at the visit, prior to conducting the procedures listed above.

In order to allow completion of the study in one visit, participants will be requested to avoid eating for one hour and ingesting caffeine for six hours prior to the testing being performed, provided ethical approval is given to allow the request for abstinence prior to the participant signing the informed consent form. If a participant has not abstained 
as required at their initial visit, informed consent may be obtained and the participant may return at a future date to complete the study visit in full, provided they have fulfilled the abstinence requirements at the second visit.

\section{General Health Questionnaire}

The health questionnaire will used to obtain the current health status of participants, including a history of cancer, cardiovascular disease, gastro-oesophageal reflux (GORD), rhinitis, sinusitis, and eczema (see appendix one). As part of the assessment of general health participants will undergo height and weight measurement to obtain their BMI. Participants will also complete a genogram in order to determine their ethnicity to three generations, including the country of birth of each generation.

\section{Respiratory Health Questionnaires}

The Asthma Quality of Life Questionnaire with Standardised Activities (AQLQ-S) is a validated 32 question self-administered questionnaire that will be used to obtain severity of asthma in the two weeks prior to study visit.

The Asthma Control Questionnaire (ACQ-5) is a validated 7 question self-administered questionnaire (only the first 5 questions are scored) that will be used to obtain severity of asthma in the 1 week prior to study visit.

\section{Lung Function Tests}

\section{Nitric Oxide}

Nitric oxide (FeNO) will be measured using a nitric oxide monitor (NIOX MINO; Aerocrine AB, Solna, Sweden) according to the ATS guidelines published in 2005. Seated subjects will exhale fully and then inhale ambient air through a nitric oxide scrubber to total lung capacity. Subjects will then exhale against an automatically adjusting resistance to achieve a constant exhalation flow rate of $50 \mathrm{ml} / \mathrm{s}+/-\mathrm{mls}$. Resistance will be adjusted so that an upper airway pressure of $10-20 \mathrm{~cm} \mathrm{H} 2 \mathrm{O}$ is maintained throughout exhalation, sufficient to close the velum and exclude nasal air. 
FeNO measurements will be taken from a stable plateau in exhaled nitric oxide concentration during an exhalation. Exhalations where flow rate and plateau criteria are not met will be deemed not acceptable for measurement. One acceptable exhalation will be measured and recorded at the visit. Measurements will be made before other pulmonary function testing. Participants will avoid eating for one hour, smoking tobacco for two hours, and caffeine ingestion for six hours prior to the testing being performed. Subjects will not be tested within three weeks of an upper or lower respiratory tract infection.

\section{Spirometry}

Participants will undergo pulmonary testing using a spirometer which meets the guidelines outlined by the American Thoracic Society 2005-Standardisation of spirometry.

\section{General guidelines}

All tests will be performed:

Sitting

With nose clips on

The tests will be clearly explained to the subject before they are started and positive encouragement given at all stages of the test.

\section{Acceptability criteria}

Criteria for technically unsatisfactory tests:

- Coughing during the first second of the exhalation

- Glottis closure that influences the measurement

- Effort that is not maximal throughout

- Obstructed mouthpiece e.g. tongue in front of the mouthpiece

- A leak in the system or around the mouthpiece

- Excessive hesitation at the start of expiration (extrapolated volume $<5 \%$ or $150 \mathrm{ml}$ of FVC -whichever is greater.

- Early termination of test by subject 


\section{Repeatability criteria}

- Less than $150 \mathrm{ml}$ variation in FEV1 between attempts

- Less than $150 \mathrm{ml}$ variation in FVC between attempts

Testing should be continued until three acceptable manoeuvres have been completed or the subject has performed eight manoeuvres - more can be done if the subject agrees. According to ATS guidelines subjects who are unable to produce reproducible flow volume loops (reproducible flow loops are those with $<150 \mathrm{ml}$ variation in FEV1 and FVC) will not be excluded from analysis. In subjects who were not able to produce three acceptable flow volume loops comments regarding the technical acceptability of their testing will be made.

\section{Laboratory Samples}

All laboratory samples will be taken by the study team at the research facilities, who are appropriately trained in phlebotomy procedures. Samples for the following tests will be analysed by a local laboratory:

- Creatinine, urea and electrolytes, serum IgE

- Full blood count (including differential white cell count)

Samples will be analysed as per the laboratory's standard operating procedures.

Samples for the following tests will be sent to the Genentech (or designee) to be analysed:

- Serum periostin level

- Optional samples for exploratory research (as per the RCR analysis below) will also be sent to Genentech (or their designee):

- Other immune mediated serum biomarkers

- DNA and RNA- based biomarker analyses 


\section{Optional Samples for Roche Clinical Repository (RCR)}

\section{Overview of the Roche Clinical Repository}

The Roche Clinical Repository (RCR) is a centrally administered group of facilities for the long-term storage of human biologic specimens, including body fluids, solid tissues, and derivatives thereof (e.g., DNA, RNA, proteins, peptides). The collection and analysis of RCR specimens will facilitate the rational design of new pharmaceutical agents and the development of diagnostic tests, which may allow for individualized drug therapy for patients in the future. Specimens for the RCR will be collected from patients who give specific consent to participate in this optional research.

\section{Approval by the Institutional Review Board or Ethics Committee}

Sampling for the RCR is contingent upon the review and approval of the exploratory research and the RCR specific Informed Consent Form (ICF) by the New Zealand Health and Disability Ethics Committees (HDEC).

\section{Optional RCR Sample Collection}

For sampling procedures, storage conditions, and shipment instructions, see the laboratory manual. RCR specimens will be destroyed no later than 15 years after the date of final closure of the associated clinical database. The RCR storage period will be in accordance with the HDEC - approved ICF and applicable laws (e.g., health authority requirements). The dynamic biomarker specimens will be subject to confidentiality standards, i.e. by coding each participant enrolled in the study through assignment of a unique subject identification number. This means that participant names are not included in data sets that are transmitted to any location outside of the research facility.

\section{Confidentiality}

Data generated from RCR specimens must be available for inspection upon request by representatives of national and local health authorities, and Roche/Genentech monitors, representatives, and collaborators, as appropriate. Participant medical information associated with RCR specimens is confidential and may only be disclosed 
to third parties as permitted by the ICF (or separate authorization for use and disclosure of personal health information) signed by the participant, unless permitted or required by law. Data derived from RCR specimen analysis on individual participants will generally not be provided to study investigators unless a request for research use is granted. The aggregate results of any research conducted using RCR specimens will be available in accordance with the effective Roche policy on study data publication. Any inventions and resulting patents, improvements, and/or know-how originating from the use of the RCR data will become and remain the exclusive and unburdened property of Roche, except where agreed otherwise.

\section{Consent to Participate in the Roche Clinical Repository}

There will be a separate ICF which will contain information that addresses participation in the RCR. The investigator or authorized designee will explain to each participant the objectives, methods, and potential hazards of participation in the RCR. Participants will be told that they are free to refuse to participate and may withdraw their specimens at any time and for any reason during the storage period. A separate, specific signature will be required to document a participant's agreement to provide optional RCR specimens. Participants who decline to participate will not provide a separate signature.

In the event of an RCR participant's death or loss of competence, the participant's specimens and data will continue to be used as part of the RCR research.

\section{Withdrawal from the Roche Clinical Repository}

Participants who give consent to provide RCR specimens have the right to withdraw their specimens from the RCR at any time for any reason. If a participant wishes to withdraw consent to the testing of his or her specimens, the investigator will inform the Genentech in writing of the participant's wishes. The participant will be provided with instructions on how to withdraw consent after the trial is closed. A participant's withdrawal from this study does not, by itself, constitute withdrawal of specimens from the RCR. Likewise, a participant's withdrawal from the RCR does not constitute withdrawal from this study. 


\section{Monitoring and Oversight}

RCR specimens will be tracked in a manner consistent with GCP by a quality-controlled, auditable, and appropriately validated laboratory information management system, to ensure compliance with data confidentiality as well as adherence to authorized use of specimens as specified in this protocol and in the ICF. Roche/Genentech and MRINZ monitors and auditors will have direct access to appropriate parts of records relating to patient participation in the RCR for the purposes of verifying the data provided to Roche, as necessary. The site will permit monitoring, audits, HDEC review, and health authority inspections by providing direct access to source data and documents related to the RCR samples.

\section{Safety Monitoring}

\section{Adverse Event Reporting}

For the purposes of this study, the study duration is considered to start once the informed consent form has been signed by the participant. The study period will be deemed to have ended when the study participant completes their study visit and leaves the research facility. The research facilities will be responsible for reviewing the blood tests taken during the study visit and reporting the clinically significant abnormalities to the participant's GP/ primary healthcare provider, as necessary.

\section{Adverse Events}

An adverse event is any untoward medical occurrence in a study subject temporally associated with the testing as part of the study. An adverse event can therefore be any unfavourable and unintended sign, symptom or disease temporally associated with the testing carried out in the study. Adverse event data will be collected by investigators during the study visit and after reviewing the test results.

\section{Serious Adverse Events (SAEs)}

For the purposes of this study the following events will be considered to be SAEs:

- Death

- Life-threatening event 
- Permanently disabling or incapacitating event

- Hospitalisation. Hospitalisation for the purposes of SAE reporting is defined as an admission to hospital and does not include a presentation to the Emergency Department followed by discharge without admission or an admission for elective reasons

- Any other significant medical event considered serious by the study investigator

All SAEs will be reported to the NZ HDEC and regulatory authorities as per current guidelines. SAEs and AEs will be followed up until resolution, or judged to be permanent. SAEs must be sent to the Sponsor within 24 hours of becoming aware of the event. SAEs will be recorded by the MRINZ (as study sponsor) within 24 hours of the study investigators becoming aware of the event.

\section{Abnormal Laboratory Values}

Not every laboratory abnormality qualifies as an adverse event. A laboratory test result should be reported as an adverse event if it meets any of the following criteria:

- Accompanied by clinical symptoms

- Results in a medical intervention (e.g., potassium supplementation for hypokalemia) or a change in concomitant therapy

- Clinically significant in the investigator's judgment

The investigators will review all laboratory findings. Medical and scientific judgment will be exercised in deciding whether an isolated laboratory abnormality should be classified as an adverse event. All relevant adverse event information will be communicated to a participant's primary health care provider as necessary.

\section{Data Safety Monitoring Committee}

Internal safety monitoring and review of safety events (adverse events and serious adverse events) will be conducted by a MRINZ non-study Researcher. 


\section{Statistical analysis}

For the non-asthmatic cohort, estimates of the mean and median periostin levels and $90 \%$ confidence intervals for prediction will be determined for both Chinese and Caucasian ethnic groups by a general linear model. As periostin has a highly skewed distribution, we will perform the analysis on the logarithm transformed scale with a back-transformation to establish the $90 \%$ confidence interval for prediction. An estimate of the difference between the Chinese and Caucasian ethnic groups will also be calculated by the general linear model. An exploratory analysis will be done to examine the effects of country of birth (New Zealand and Australia vs Asia).

The sample size of 120 adults is based on the recommendations of the Clinical and Laboratory Standards Institute that 120 participants should be studied to allow $90 \%$ confidence intervals to be computed by non-parametric methods if normal distribution assumptions are not met. No stratification by age or sex will be undertaken, as there is no association between these variables and serum periostin levels. Based on the SD of the logarithm periostin of 0.22 , a sample size of 120 in the Chinese group and 420 in the Caucasian group has $90 \%$ power with alpha $5 \%$ to detect a difference in mean logarithm periostin of 0.074 equivalent to a ratio of mean periostin of 1.08 .

With regards to investigating if an association exists between asthma severity and serum periostin levels in a Chinese population, 68 participants are sufficient to detect a paired difference in periostin of 0.5 of a standard deviation with $80 \%$ power. The conventional interpretation of an effect size of 0.5 of a standard deviation is of a medium effect size. For a comparison between mild and moderate/severe asthma, a sample size of 68 has $90 \%$ power to detect a 0.8 standard deviation difference, a large effect size difference. 


\title{
Appendix VII: Participant Information Sheet for Study Four
}

\author{
Participant Information Sheet
}

Study title: Longitudinal study of serum periostin levels in asthma (PER02)

Locality: Medical Research $\quad$ Ethics 13/NTB/185

Institute of New Zealand committee ref.:

Lead Prof. Richard Beasley Contact phone 048050147

investigator: number:

You are invited to take part in a study to determine the change in periostin levels over eight weeks in group of patients, aged 18-75 years, with stable asthma. Whether or not you take part is your choice. If you don't want to take part, you don't have to give a reason, and it won't affect the care you receive. If you do want to take part now, but change your mind later, you can pull out of the study at any time.

You will not be eligible to take part in this study if any of the following applies to you:

- You have had a change in asthma medication in the last 3 months

- You are known to be pregnant

- You have been admitted to hospital or had surgery in the last 3 months

- You have had a bone fracture in the last 3 months

- You have had oral or systemic steroids in the last 3 months.

This Participant Information Sheet will help you decide if you'd like to take part. It sets out why we are doing the study, what your participation would involve, what the benefits and risks to you might be, and what would happen after the study ends. We will go through this information with you and answer any questions you may have. We expect this will take about 20 minutes. You may also want to talk about the study with 
other people, such as family, whānau, friends, or healthcare providers. Feel free to do this.

If you agree to take part in this study, you will be asked to sign the Consent Form on the last page of this document. You will be given a copy of both the Participant Information Sheet and the Consent Form to keep.

This document is 8 pages long, including the Consent Form. Please make sure you have read all the pages. If you require an interpreter, this will be arranged.

\section{Why are we doing the study?}

The protein periostin has been found to be present in a number of medical conditions, including asthma, early bone healing, progression of various cancers and kidney disease related to high blood pressure. It is easily measured (by a blood test) and in the future it may be used to determine the status of a person's disease, including its severity, if it is progressing or if it is no longer present. Proteins like periostin that can allow doctors to assess a disease are known as biomarkers. This study is being conducted to determine changes in periostin levels over time in patients with asthma which will allow this disease to be better monitored and treated in the future.

We aim to have a total of 60 participants involved in this study.

The Sponsor of this study is the Medical Research Institute of New Zealand. The study is being funded by Genentech Inc, a member of the Roche Group.

The study has been approved by the Northern B Health and Disability Ethics Committee, reference number 13/NTB/185. 


\section{What would your participation involve?}

The study is being conducted at the Medical Research Institute of New Zealand, based at Wellington Hospital. If you agree to be a part of the study you will need to attend the Institute for a total of 11 visits. Visit 1 will take up to an hour and the remaining visits will be approximately fifteen minutes each.

\section{Visit Outline}

\section{Visit 1}

After reading this information sheet and signing the informed consent form, the study investigator will provide you with a general health questionnaire which you will fill out in their presence. The study investigator will then discuss your medical history and current medications so that a full picture is gained of your current health. If required we will contact your GP (with your permission) to clarify aspects of your medical history or current medications. You will be asked to bring in your current medications and to withhold the following prior to the breathing tests:

- Withhold caffeine for 6 hours prior to your appointment.

- Withhold cigarettes for 2 hours prior to your appointment

- Withhold food for 1 hour prior to your appointment

You will be given two asthma questionnaires (AQLQ and ACQ-5) which you will fill out in the presence of the study investigator.

The following tests will then be carried out:

We will measure nitric oxide (a gas that you normally breathe out) by asking you to breathe into a tube.

To check the health and function of your lungs you will be asked to do some breathing exercises. You will then follow the instructions given to you by the investigator, for a few minutes. This test will be done so that 3 of your best measurements are recorded. This may take up to 8 measurements (or more if you agree) in total, so that we can record the best results. 
We will take a small blood sample, to measure your full blood count, serum IgE and serum periostin level.

If for any reason you cannot complete the tests (e.g. due to not withholding caffeine etc) at the first visit, you can go through the consent process and can attend for visit $1 \mathrm{~b}$ and complete the testing on a different day.

Visit 2-11

You will be asked about your current health and any changes to your health since your last visit.

A blood sample will be taken to measure your periostin level at visits 2-11.

A blood sample will also be taken at visits $2-7$, so that we can measure your full blood count.

At visits 6-11 we will ask you to complete the ACQ-5 questionnaire.

What are the possible benefits and risks to you of participating?

\section{Benefits}

There will be no direct benefit to you in participating in this study, you will however be part of research which may benefit yourself and others in the future.

\section{Study Procedure Risks/ Side Effects}

\section{Blood Samples}

You may experience some discomfort during the taking of a blood sample and there is always the risk of bleeding, swelling and bruising at the site of the needle during sampling. All samples will be taken by trained staff. Around 1-2 tablespoons of blood will be taken at each visit. In some cases we may require extra samples, for example to re-do a test that could not be analysed. A local laboratory will analyse your full blood count, as well as serum IgE. Periostin will be analysed at a central laboratory outside of New Zealand, by Genentech. This laboratory is:

Covance Central Laboratory Services 


\section{Scicor Drive}

Indianapolis, Indiana 46214

USA

Your blood samples will be coded with a unique subject number and your name will not be used to identify the samples. The samples analysed locally will be destroyed by the laboratory once the results are reported. The samples sent to Genentech may be stored for 5 years after the study. You may hold beliefs about a sacred and shared value of all or any tissue samples removed. The cultural issues associated with sending your samples overseas and/or storing your tissue and/or undertaking genetic analysis on them should be discussed with your family/whānau as appropriate. There are a range of views held by Māori around these issues; some iwi disagree with storage of samples and genetic testing citing whakapapa and advise their people to consult prior to participation in research where this occurs. However it is acknowledged that individuals have the right to choose.

\section{Breathing Exercises}

You may feel some discomfort during or after performing the breathing exercises, however this will be transient and you will be monitored constantly throughout the tests by trained technicians/ study investigators. You will be seated at all times for the tests.

\section{Cost}

There will be no cost to you to take part in this study and you will be compensated for your time and travel expenses to attend your study visits ( $\$ 45$ for visit 1 and $\$ 20$ for visits 2-11).

\section{General Health Care}

Your general health care remains with your GP throughout the time you are in the study and if you experience any problems you should contact your usual health care provider. As part of the informed consent process we will ask for your permission to inform your GP of any unexpected clinically significant findings. As part of the study 
you will be seen by study investigators, study doctors and trained staff who will conduct all the study specific assessments.

\section{What would happen if you were injured in the study?}

If you were injured as a result of treatment given as part of this study, which is unlikely, you won't be eligible for compensation from ACC. However, compensation would be available from the study's sponsor (Medical Research Institute of New Zealand), in line with current guidelines. We can give you a copy of these guidelines if you wish. You would be able to take action through the courts if you disagreed with the amount of compensation provided.

If you have private health or life insurance, you may wish to check with your insurer that taking part in this study won't affect your cover.

\section{What are the rights of participants in the study?}

Your participation is entirely voluntary (your choice). You do not have to take part in this study, and if you choose not to take part it will in no way affect your future health care. If you do agree to take part you are free to withdraw from the study at any time, without having to give a reason.

\section{Confidentiality and Data Privacy}

If you decide to participate, the study doctor and relevant Institute staff will collect medical and personal information about you, as part of doing the study. By agreeing to take part in this research, you will allow your medical information and results to be seen by people who check that the research was done properly, for example the ethics committee and study monitor. Nothing which could personally identify you will be used in any reports on this study, or provided as part of future studies. Your personal information (for example name, sex, age and medical conditions) and other information will be identified by a study number (i.e. coded). 
The study records will be stored securely during the course of the study and once it is completed. After all participants have completed the study and the data has been monitored, the records will be archived for a minimum of 10 years as per New Zealand regulations. The records will then be securely destroyed.

What will happen after the study ends, or if you pull out?

Once the study has finished and the data has been analysed you will be able to find out the results from the study. You may also request a copy of your individual lung function results.

The sponsor may stop the study at any time. You may be asked to leave the trial, for the following reasons:

- In the Investigator's opinion it would not be in your best interest to continue in the study

- Any safety concerns

- You do not follow instructions during the study visits

Where can you go for more information about the study, or to raise concerns or complaints?

If you have any questions, concerns or complaints about the study at any stage, you can contact the study investigator:

$\begin{array}{ll}\text { Name: } & \text { Dr Rachel Caswell-Smith } \\ \text { Phone: } & 8050247 \\ \text { E-mail: } & \text { rachel.caswell-smith@mrinz.ac.nz }\end{array}$

For Maori health support, please contact:

Whānau Care Services, Cultural Care Centre, Level 2, Wellington Hospital

Phone: $\quad 0800999442$ or 048060948 


\section{Email: $\quad$ wcs@ccdhb.org.nz}

If you want to talk to someone who isn't involved with the study, you can contact an independent health and disability advocate on:

Phone: $\quad 0800555050$

Fax: $\quad 08002$ SUPPORT (0800 2787 7678)

Email: $\quad$ advocacy@hdc.org.nz

You can also contact the health and disability ethics committee (HDEC) that approved this study on:

Phone: $\quad 08004$ ETHICS (0800 4384 427)

Email: $\quad \underline{\text { hdecs@moh.govt.nz }}$ 


\section{Consent Form}

Study title: Longitudinal study of serum periostin levels in asthma (PER02)

Participant Number:

Please tick to indicate you consent to the following:

\begin{tabular}{|c|}
\hline $\begin{array}{l}\text { I have read (or have had read to me in a language I understand) and understood the } \\
\text { Participant Information Sheet }\end{array}$ \\
\hline I have been given sufficient time to consider whether or not to participate in this study. \\
\hline $\begin{array}{l}\text { I have had the opportunity to use a legal representative, whānau/ family support or a } \\
\text { friend to help me ask questions and understand the study }\end{array}$ \\
\hline $\begin{array}{l}\text { I am satisfied with the answers I have been given regarding the study and I have a copy } \\
\text { of this consent form and information sheet. }\end{array}$ \\
\hline $\begin{array}{l}\text { I understand that taking part in this study is voluntary (my choice) and that I may } \\
\text { withdraw from the study at any time without this affecting my medical care. }\end{array}$ \\
\hline $\begin{array}{l}\text { I consent to the research staff collecting and processing my information, including } \\
\text { information about my health }\end{array}$ \\
\hline I consent to my blood sample for Periostin to be sent overseas for storage and analysis \\
\hline $\begin{array}{l}\text { If I decide to withdraw from the study, I agree that the information collected about me } \\
\text { up to the point when I withdraw may continue to be processed }\end{array}$ \\
\hline $\begin{array}{l}\text { I consent to my GP/ usual health care provider being informed of any significant } \\
\text { abnormal results obtained during the study }\end{array}$ \\
\hline I agree to an approved auditor appointed by the New Zealand Health and Disability Ethic \\
\hline $\begin{array}{l}\text { Committees, or any relevant regulatory authority or their approved representative } \\
\text { reviewing my relevant medical records for the sole purpose of checking the accuracy of }\end{array}$ \\
\hline $\begin{array}{l}\text { I understand that my participation in this study is confidential and that no material, } \\
\text { which could identify me personally, will be used in any reports on this study. }\end{array}$ \\
\hline I understand the compensation provisions in case of injury during the study. \\
\hline I know who to contact if I have any questions about the study in general \\
\hline \\
\hline
\end{tabular}

\begin{tabular}{|l|l|l|}
\hline \multicolumn{1}{|c|}{ Yes No } \\
\hline I wish to receive a summary of the results from the study. & & \\
\hline I give consent for study investigators to contact my GP/ usual health care \\
provider to clarify my medical history or current medications, if required & & \\
\hline
\end{tabular}


I give consent for study investigators to inform my GP/ usual health care provider of my participation in this study

Declaration by participant:

I hereby consent to take part in this study

Participant name (print)

Participant signature

Date

Declaration by member of research team:

I have given a verbal explanation of the research project to the participant, and have answered the participant's questions about it.

I believe that the participant understands the study and has given informed consent to participate.

$\overline{\text { Name of person conducting informed consent discussion }}$

(print)

Signature of person conducting informed consent
discussion




\title{
Appendix VIII: Protocol for Study Four
}

\author{
Protocol \\ Longitudinal study of serum periostin levels in asthma \\ Protocol No.: PERO2 \\ UTN: U1111-1150-0071
}

ANZCTRN: ACTRN12614000235606

\section{Rationale}

Periostin is an extracellular matrix protein encoded by the POSTN gene (an IL-4/ IL-13 inducible gene) thought to be an adhesion molecule because it possesses four fasciclin I domains. ${ }^{16}$ Periostin has been shown to be secreted in the extracellular matrix from the basolateral surface of bronchial epithelial cells after stimulation with IL-13. ${ }^{98}$

Periostin has been studied in patients with asthma, expression levels of the POSTN gene corresponding to reticular basement membrane thickness an indicator of subepithelial fibrosis. ${ }^{98}$ Periostin is thought to enhance fibrosis in the airways by binding to other extracellular proteins collagen, fibronectin and tenascin C. ${ }^{241}$ Periostin may also have a role in other $\mathrm{TH} 2$ driven inflammation such as nasal polyps, eosinophilic oesophagitis, allergic rhinitis and atopic dermatitis.

A highly specific serum assay for periostin has been produced which allows levels of periostin in peripheral blood to be tested. Through use of this assay periostin has been identified as a systemic biomarker for airway eosinophilia. Periostin levels in peripheral blood correspond to eosinophilic inflammation in tissue taken from the airways of patients with asthma. ${ }^{98}$ Using periostin as a systemic biomarker of airway eosinophilia would provide a minimally invasive monitoring technique which could be used to monitor severity, response to treatment and determine treatment options for patients with asthma. ${ }^{98,241}$ In order to use serum periostin levels as a biomarker in asthma a more complete picture of periostin production in asthma needs to be 
formed. This study of the longitudinal variation of periostin in patients with stable asthma would provide further information on the production of periostin in asthma. Until now the periostin assay has only been used to determine the relationship of periostin with eosinophilic airway inflammation in patients not responding to inhaled steroid treatment and has not been studied in patients with stable, well controlled asthma. ${ }^{241}$

This study has been initiated and designed by the Medical Research Institute of New Zealand in collaboration with Genentech, Inc (a member of the Roche group). The Institute is the Sponsor of this study and undertakes Sponsor responsibilities for the purposes of ethical and any applicable regulatory requirements. Genentech are fully funding the study.

\section{Objective}

To determine the eight-week longitudinal variation in serum periostin level in a population with stable asthma.

\section{Outcomes}

Primary outcome measure:

- Longitudinal variation in serum periostin level over an eight-week period

\section{Secondary outcome measures:}

Investigation of potential associations between serum periostin, eosinophils and the following:

- AQLQ score (from respiratory health questionnaire)

- Non-asthmatic health conditions (as captured by general health questionnaire e.g. diabetes)

- Spirometry (FEV1 and FVC)

- Fractional exhaled Nitric Oxide (FeNO) level

- Serum IgE 
Investigation of potential associations between serum periostin and eosinophils

- Exploratory outcome measures

- Other asthma related biomarkers

- RNA- based biomarker analyses

- Variation of eosinophils

\section{Study design}

Participants:

60 Participants with a doctor's diagnosis of asthma on regular medication (including maintenance inhaled corticosteroid and long acting beta agonists) primarily recruited from the Medical Research Institute of New Zealand (MRINZ) databases. If required further recruitment will take place via referrals from other medical professionals, or through direct advertising.

\section{Inclusion Criteria}

- Aged between 18 to 75 years

- Able to provide written informed consent

- Doctor diagnosis of asthma

- On a stable asthma treatment regime for $\geq 3$ months prior to visit 1 , taking regular inhaled medication for asthma (including maintenance inhaled corticosteroid and long acting beta agonists)

\section{Exclusion Criteria}

- Unstable asthma (as determined by the investigator and/or a change in asthma medication in last 3 months)

- Oral or Systemic Corticosteroids within the last 3 months

- Known Pregnancy*

- Significant co morbidities (determined at investigator discretion)

- Hospital admission within last 3 months 
- Surgery within last 3 months, determined at investigator discretion (this includes all major surgery requiring general anaesthetic, dental extractions and root canal procedures. This does not include minor procedures, including but not limited to mole or wart removal, dental fillings etc).

- Bone fracture within the last 3 months

- Active (current, or within the 3 weeks prior to the visit) upper or lower respiratory tract infection

- Any other safety concern at the investigator's discretion

* If a potential subject is aware they are currently pregnant, or actively trying to conceive, they will be excluded from the study as pregnancy can affect FeNO, eosinophil and spirometry results. No pregnancy test will be performed on women of childbearing potential as there are no safety concerns relating to this study.

Participants may be re-screened at a suitable time should it become apparent they cannot fulfil eligibility criteria at their initial visit (for example they are experiencing a respiratory infection).

Following screening, 60 participants will be enrolled, from which there will be anticipated maximal $20 \%$ withdrawal or exclusion rate. As a result data will be collected on at least 50 participants.

\section{Study Procedures}

\section{Study Visits}

Participants will attend the MRINZ for a total of 11 visits.

Visits will be daily for 5 days, weekly for 4 weeks and then at 8 weeks. (Day 1, 2, 3, 4, 5, $10,17,24,31,38,59)$. There will be a visit window of $+/-1$ day. If a visit is delayed the calculated schedule of visit dates from baseline (visit $1 \mathrm{~b}$ ) shall be maintained. Any delays greater than $+/-1$ day will be recorded as protocol deviations.

Visit $1 a$ 
Written Informed Consent

Visit $1 b$

- Completion of General Health Questionnaire

- Completion of Respiratory Health Questionnaire AQLQ

- Completion of Respiratory Health Questionnaire ACQ-5

- Measurement of Body Mass Index (BMI)

- Spirometry assessment (Measurement of FEV ${ }_{1}$ and FVC)

- Measurement of FeNO

- Laboratory samples for measurement of:

- Full blood count (including differential white cell count)

- Serum lgE

- Serum periostin level

- Serum and RNA- based biomarker analyses will also be conducted on samples collected for the Roche Clinical Repository (RCR). These samples will only be collected from participants who provide consent on the RCR informed consent form which is a separate consent to the main study (see Optional samples for Roche Clinical Repository).

Visits $1 \mathrm{a}$ and $1 \mathrm{~b}$ will be combined if the participant meets the testing criteria for the FeNO testing and spirometry (outlined below - avoidance of caffeine, tobacco, and food)

Visit 2 -11

- Laboratory samples for measurement of:

- Periostin level at visits 2-11

- Serum and RNA based biomarker analyses will also be conducted on samples collected for the Roche Clinical Repository (RCR). These samples will only be collected from participants who provide consent on the RCR informed consent form which is a separate consent to the main study (see Optional samples for Roche Clinical Repository) at visits 2-11 
- A full blood count with differential cell count (for eosinophil measurement) at visits $2-7$

- ACQ-5 will be administered at visits 6-11.

\section{Informed Consent}

Informed consent will be obtained according to ethical and GCP guidelines, and will take place at the MRINZ. Each participant will sign an informed consent form at the first visit, prior to conducting the procedures listed above.

\section{Respiratory Health Questionnaires}

The Asthma Quality of Life Questionnaire with Standardised Activities (AQLQ(S)) is a validated 32 question self administered questionnaire that will be used to obtain severity of asthma in the two weeks prior to visit 1(b).

The Asthma Control Questionnaire (ACQ5) is a validated 5 question self-administered questionnaire that will be used to obtain severity of asthma in the 1 week prior to visit 1.

\section{General Health Questionnaire}

The health questionnaire will be used to obtain the current health status of participants, including a history of cancer, cardiovascular disease, gastro-oesophageal reflux (GORD), rhinitis, sinusitis, and eczema (see appendix 1). As part of the assessment of general health participants will undergo height and weight measurement to obtain their BMI.

\section{Lung Function Tests}

Nitric Oxide

Nitric oxide (FeNO) will be measured using a nitric oxide monitor (NIOX MINO; Aerocrine AB, Solna, Sweden) according to the ATS guidelines published in 2005. Seated subjects will exhale fully and then inhale ambient air through a nitric oxide scrubber to total lung capacity. Subjects will then exhale against an automatically adjusting resistance to achieve a constant exhalation flow rate of $50 \mathrm{ml} / \mathrm{s}+/-\mathrm{mls}$. Resistance will be adjusted so that an upper airway pressure of $10-20 \mathrm{~cm} \mathrm{H} 2 \mathrm{O}$ is 
maintained throughout exhalation, sufficient to close the velum and exclude nasal air. FeNO measurements will be taken from a stable plateau in exhaled nitric oxide concentration during an exhalation. Exhalations where flow rate and plateau criteria are not met will be deemed not acceptable for measurement. One acceptable exhalation will be measured and recorded at each time point. Measurements will be made before other pulmonary function testing. Participants will avoid eating for 1 hour, smoking tobacco for 2 hours, and caffeine ingestion for 6 hours. Subjects will not be tested within 3 weeks of an upper or lower respiratory tract infection.

\section{Spirometry}

Participants will undergo pulmonary testing using a spirometer which meets the guidelines outlined by the American Thoracic Society 2005-Standardisation of spirometry.

\section{General guidelines}

All tests will be performed:

Sitting

With nose clips on

The tests will be clearly explained to the subject before they are started and positive encouragement given at all stages of the test.

\section{Acceptability criteria}

Criteria for technically satisfactory tests:

- Coughing during the first second of the exhalation

- Glottis closure that influences the measurement

- Effort that is not maximal throughout

- Obstructed mouthpiece e.g. tongue in front of the mouthpiece

- A leak in the system or around the mouthpiece

- Excessive hesitation at the start of expiration (extrapolated volume $<5 \%$ or $150 \mathrm{ml}$ of FVC -whichever is greater.

- Early termination of test by subject 
Repeatability criteria

- Less than $150 \mathrm{ml}$ variation in FEV1 between attempts

- Less than $150 \mathrm{ml}$ variation in FVC between attempts

Testing should be continued until 3 acceptable manoeuvres have been completed or the subject has performed 8 manoeuvres-more can be done if the subject agrees. According to ATS guidelines subjects who are unable to produce reproducible flow volume loops (reproducible flow loops are those with $<150 \mathrm{ml}$ in variation in FEV1 and FVC) will not be excluded from analysis. In subjects who were not able to produce 3 acceptable flow volume loops comments regarding the technical acceptability of their testing will be made.

\section{Laboratory Samples}

Samples for the following tests will be analysed by a local laboratory:

- Serum IgE

- Full blood count (including differential white cell count)

Samples will be analysed as per the laboratory's standard operating procedures.

Samples for the following tests will be sent to Genentech (or designee) to be analysed:

- Serum periostin level

- Optional serum and RNA- based biomarker analyses (as per the RCR below)

\section{Optional Samples for Roche Clinical Repository (RCR)}

Overview of the Roche Clinical Repository

The Roche Clinical Repository (RCR) is a centrally administered group of facilities for the long-term storage of human biologic specimens, including body fluids, solid tissues, and derivatives thereof (e.g., DNA, RNA, proteins, peptides). The collection and analysis of RCR specimens will facilitate the rational design of new pharmaceutical agents and the development of diagnostic tests, which may allow for individualized drug therapy for patients in the future. 
Specimens for the RCR will be collected from patients who give specific consent to participate in this optional research.

\section{Approval by the Institutional Review Board or Ethics Committee}

Sampling for the RCR is contingent upon the review and approval of the exploratory research and the RCR specific Informed Consent Form (ICF) by the New Zealand Health and Disability Ethics Committees.

\section{Optional RCR Sample Collection}

For sampling procedures, storage conditions, and shipment instructions, see the laboratory manual. RCR specimens will be destroyed no later than 15 years after the date of final closure of the associated clinical database. The RCR storage period will be in accordance with the IRB/EC-approved ICF and applicable laws (e.g. health authority requirements). The dynamic biomarker specimens will be subject to confidentiality standards, i.e. by coding each participant enrolled in the study through assignment of a unique subject identification number. This means that participant names are not included in data sets that are transmitted to any location outside of the MRINZ.

\section{Confidentiality}

Data generated from RCR specimens must be available for inspection upon request by representatives of national and local health authorities, and Roche monitors, representatives, and collaborators, as appropriate.

Participant medical information associated with RCR specimens is confidential and may only be disclosed to third parties as permitted by the ICF (or separate authorization for use and disclosure of personal health information) signed by the participant, unless permitted or required by law. Data derived from RCR specimen analysis on individual participants will generally not be provided to study investigators unless a request for research use is granted. The aggregate results of any research conducted using RCR specimens will be available in accordance with the effective Roche policy on study data publication. Any inventions and resulting patents, improvements, and/or know-how originating from the use of the RCR data will become and remain the exclusive and unburdened property of Roche, except where agreed otherwise. 


\section{Consent to Participate in the Roche Clinical Repository}

There will be a separate ICF which will contain information that addresses participation in the RCR. The investigator or authorized designee will explain to each participant the objectives, methods, and potential hazards of participation in the RCR. Participants will be told that they are free to refuse to participate and may withdraw their specimens at any time and for any reason during the storage period. A separate, specific signature will be required to document a participant's agreement to provide optional RCR specimens. Participants who decline to participate will not provide a separate signature. In the event of an RCR participant's death or loss of competence, the participant's specimens and data will continue to be used as part of the RCR research.

\section{Withdrawal from the Roche Clinical Repository}

Participants who give consent to provide RCR specimens have the right to withdraw their specimens from the RCR at any time for any reason. If a participant wishes to withdraw consent to the testing of his or her specimens, the investigator will inform Genentech in writing of the participant's wishes. The participant will be provided with instructions on how to withdraw consent after the trial is closed. A participant's withdrawal from this study does not, by itself, constitute withdrawal of specimens from the RCR. Likewise, a participant's withdrawal from the RCR does not constitute withdrawal from this study.

\section{Monitoring and Oversight}

RCR specimens will be tracked in a manner consistent with Good Clinical Practice (GCP) by a quality-controlled, auditable, and appropriately validated laboratory information management system, to ensure compliance with data confidentiality as well as adherence to authorized use of specimens as specified in this protocol and in the ICF. Roche and MRINZ monitors and auditors will have direct access to appropriate parts of records relating to patient participation in the RCR for the purposes of verifying the data provided to Roche, as necessary. The site will permit monitoring, audits, IRB/EC review, and health authority inspections by providing direct access to source data and documents related to the RCR samples. 


\section{Safety Monitoring}

Adverse Event Reporting

For the purposes of this study, the study duration is considered to start once the informed consent form has been signed by the participant. The study period will be deemed to have ended when the study participant completes their final study visit (visit 12) and leaves MRINZ premises. The MRINZ will be responsible for reviewing the blood tests taken during the study visits and reporting any clinically significant abnormalities to the participant's GP/ primary healthcare provider, as necessary.

\section{Adverse Events}

An adverse event is any untoward medical occurrence in a study subject temporally associated with the testing as part of the study. An adverse event can therefore be any unfavourable and unintended sign, symptom or disease temporally associated with the testing carried out in the study. Adverse event data will be collected by investigators during the study visits and after reviewing the test results.

\section{Serious Adverse Events (SAEs)}

For the purposes of this study the following events will be considered to be SAEs:

- Death

- Life-threatening event

- Permanently disabling or incapacitating event

- Hospitalisation. Hospitalisation for the purposes of SAE reporting is defined as an admission to hospital and does not include a presentation to the Emergency Department followed by discharge without admission or an admission for elective reasons

- Any other significant medical event considered serious by the study investigator

All SAEs will be reported to the NZ HDEC and regulatory authorities as per current guidelines. SAEs and AEs will be followed up until resolution, or judged to be 
permanent. All SAEs will be recorded by the MRINZ (as study sponsor) within 24 hours of the study investigators becoming aware of the event.

\section{Abnormal Laboratory Values}

Not every laboratory abnormality qualifies as an adverse event. A laboratory test result should be reported as an adverse event if it meets any of the following criteria:

- Accompanied by clinical symptoms

- Results in a medical intervention (e.g., potassium supplementation for hypokalemia) or a change in concomitant therapy

- Clinically significant in the investigator's judgment

The investigators will review all laboratory findings. Medical and scientific judgment will be exercised in deciding whether an isolated laboratory abnormality should be classified as an adverse event. All relevant adverse event information will be communicated to a participant's primary health care provider as necessary.

\section{Data Safety Monitoring Committee}

Internal safety monitoring and review of safety events will be conducted by a Medical Research Institute non-study Researcher.

\section{Data Handling/ Statistical Methods}

\section{Study Power:}

60 Participants will be recruited to the study. Assuming at most $20 \%$ withdrawal/exclusion rates during the study this will ensure complete data on at least 50 participants. The recent three-month inhaled steroid intervention trial conducted as part of the NZRHS had a $12 \%$ withdrawal rate and few severe exacerbations. An achieved sample size of 50 has reasonable precision for estimates of variance.

\section{Statistical analysis:}

The baseline characteristics of the population will be described and the variance and mean values for serum periostin will be calculated for participants who complete the 
study without developing a severe exacerbation. Confidence intervals for the variance of periostin will be calculated using a Chi-square statistic. This analysis may need to be performed on the log transformed scale. 


\section{Appendix IX: General Health}

\section{Questionnaire}

\section{Periostin Reference Range General Health Questionnaire}

Participant ID:

Name:

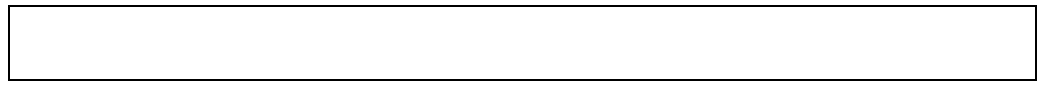

Date:

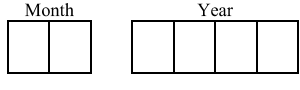

A. General Questions

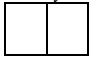

1. Date of Birth

Age
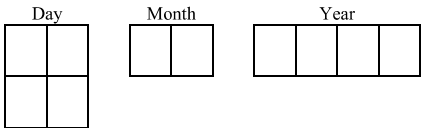

2. Which ethnic group do you belong to? Mark the space or spaces which apply to you.

\section{Maori}

New Zealand European

Samoan

Cook Island Maori

Tongan

Niuean

Chinese

Indian

Other (such as DUTCH, JAPANESE, TOKELAUAN). Please state:

3. Are you male or female?

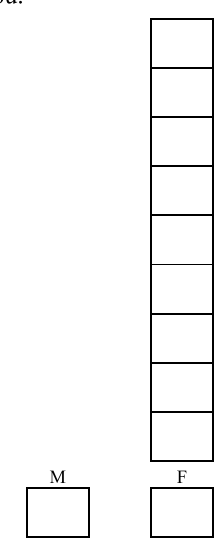

B. Smoking History

4a. Have you ever smoked cigarettes?

4b. Do you currently smoke cigarettes?

4c. If NO, date of last cigarette:
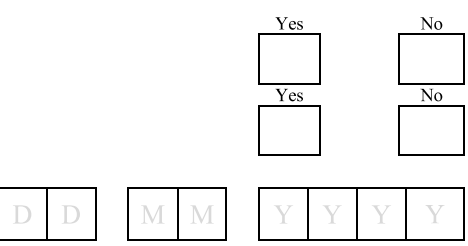

4d. Number of years as smoker

4e. Number of cigarettes smoked per day on average:

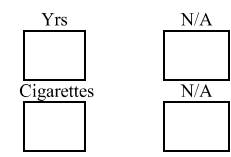

Investigator calculate pack years

PER01 General Health Questionnaire

Version $1(06 / 11 / 2013)$ 


\section{Exercise}

5. In the last 7 days have you exercised for $>30$ minutes at a time and had to breathe hard.

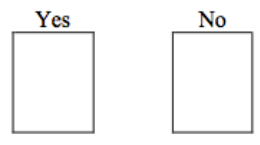

If NO, please go to question 6, if YES, proceed with this section:
5a. Date of last exercise
5b. Time of last exercise

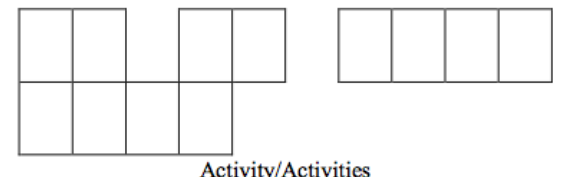

5c. Exercise Activity

\section{Respiratory}

10a. Have you ever been diagnosed with asthma, chronic bronchitis, COPD, emphysema or bronchiectasis?

10b. Have you experienced any wheezing or whistling in the chest in the past 12 months?

10c. Have you ever been admitted to hospital or required surgery for a condition relating to your lungs?

Please Specify:

\section{E. Eyes}

6a. Do you ever experience itchy or watery eyes?

Please Specify:

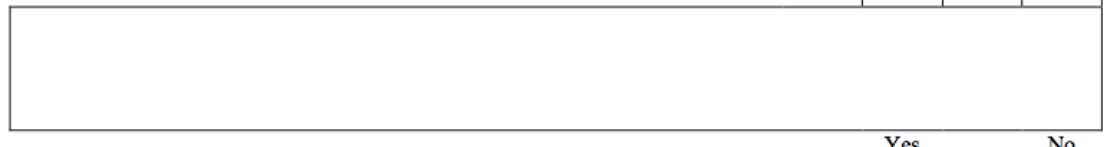

6b. Have you been diagnosed with an eye condition?

Please Specify: 


\section{F. Ear, Nose and Throat}

7a. Do you experience symptoms of Rhinitis (runny/stuffy, itchy nose,

Runny

Nose sneezing) not associated with a cold/flu? Please circle

7b. Have you been diagnosed with nasal polyps?

7c. Do you have a known tooth cavity?

7d. Have you had a dental procedure in the last 3 months?

7e. Have you been diagnosed with an ear, nose or throat condition? Please Specify:
Stuffy

Sneezing
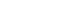

\section{G. Gastrointestinal}

8a. Do you have Gastroesophageal Reflux Disease (GORD) or reflux?

8b. Do you have eosinophilic oesophagitis?

8c. Do you have inflammatory bowel disease (crohn's disease, ulcerative colitis)? Please Specify:

8d. Have you been diagnosed with a condition relating to your digestive tract (oesophagus, stomach or bowel)?

Please Specify:

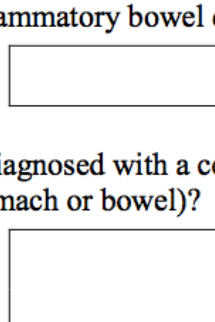




\section{H. Cardiovascular}

9a. Have you been diagnosed with high blood pressure?

9b. Have you ever been diagnosed with high cholesterol?

9c. Do you experience chest pain/angina?

9d. Have you ever had a heart attack (myocardial infarction)?

9e. Have you ever been diagnosed with heart failure?

9f. Have you ever been diagnosed with problem with your heart valve(s)?

9g. Have you ever been admitted to hospital for a heart condition or had cardiac surgery?

Please Specify:
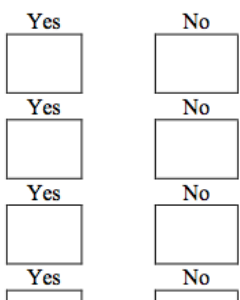

\begin{tabular}{ll} 
Yes & No \\
& \\
\hline
\end{tabular}

Yes No

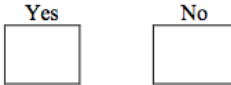

Yes No

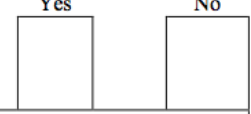

I. Genitourinary

11a. Have you ever been diagnosed with kidney disease?

Please Specify:

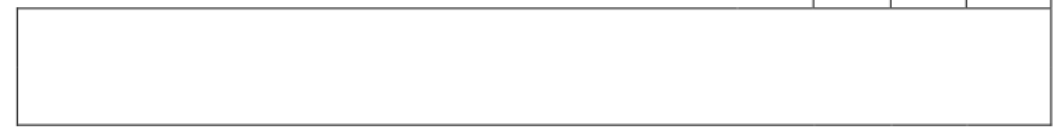

11b. FEMALES: Are you using contraception?

Please Specify:

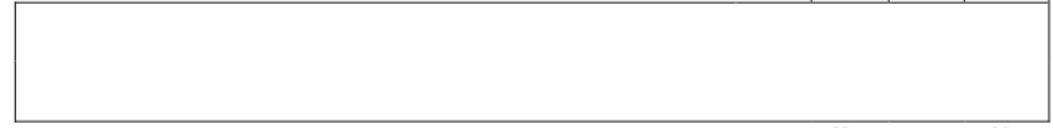

11c. FEMALES: Are you post menopausal?

Date of last Period:

PER01 General Health Questionnaire

Version $1(06 / 11 / 2013)$ 


\section{J. Musculoskeletal}

12a. Have you ever been diagnosed with osteoarthritis?

12b. Have you ever been diagnosed with inflammatory arthritis? (rheumatoid arthritis, ankylosing spondylitis, psoriatic arthritis)
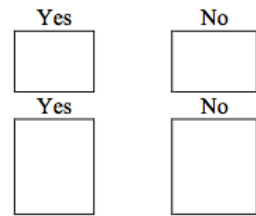

12c. Have you ever been diagnosed with osteoporosis?

12d. Have you ever broken a bone?

Date of most recent Fracture:

Site of most recent Fracture:

12e. Have you ever had a joint replacement?

Date of Replacement:

Site of Replacement:

\section{K. Skin}

13a. Have you ever been diagnosed with eczema?

13b. Have you ever been diagnosed with psoriasis?
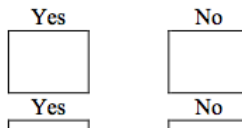

13c. Do you have a current open wound?

Date of Injury:

Site of Wound:

\section{Neurological}

14. Have you ever been diagnosed with a neurological condition?

Please Specify:

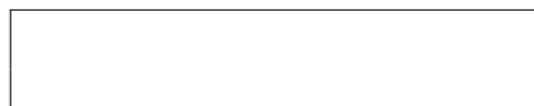




\section{Psychiatric}

15. Have you ever been diagnosed with a psychiatric or mental health condition? Please Specify:

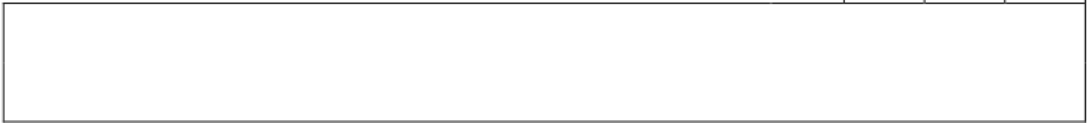

\section{N. Endocrine}

16a. Have you ever been diagnosed with a Thyroid condition? Please Specify:

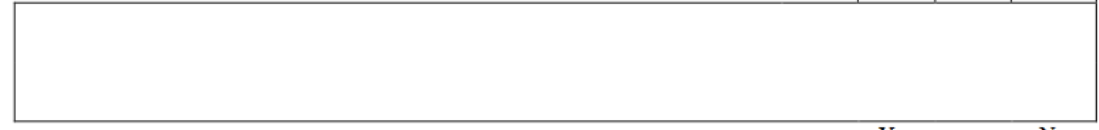

16b. Have you ever been diagnosed with diabetes?

16c. Have you ever been diagnosed with impaired glucose tolerance or prediabetes?

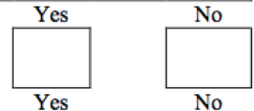

16d. Have you ever been diagnosed with any other endocrine condition?

Please Specify:

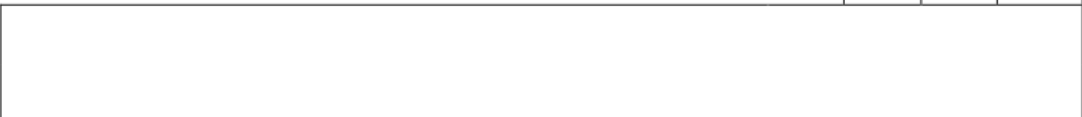

\section{O. Haematology}

17a. Have you ever been diagnosed with a blood disorder? eg anaemia, haemochromatosis, leukaemia

Please Specify:

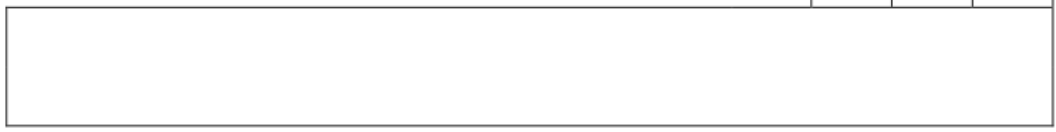

\section{P. Cancer}

18a. Have you ever been diagnosed with cancer?

Please Specify site:

Date:

Treatment: 


\section{Q. Allergies}

19. Do you have any allergies?

Please Specify:

\section{R. Medications}

Investigator:

Please document all medications in visit worksheet

Medications reconciled to medical history?

1

0

PER01 General Health Questionnaire

Version $1(06 / 11 / 2013)$ 
Appendix X: Asthma Control

Questionnaire (ACQ) 


\section{ASTHMA CONTROL QUESTIONNAIRE (ACQ)}

\section{ENGLISH VERSION FOR NEW ZEALAND}

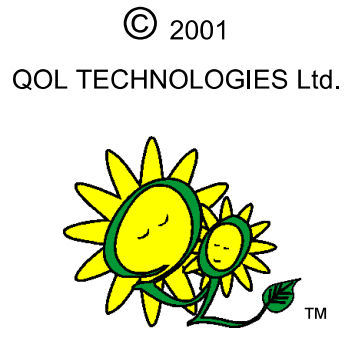

For further information:

Elizabeth Juniper, MCSP, MSc

Professor

20 Marcuse Fields

Bosham, West Sussex

P018 8NA, England

Telephone: +44 1243572124

Fax: +44 1243573680

E-mail: juniper@qoltech.co.uk

Web: http://www.qoltech.co.uk

This translation has been made possible through a grant from GLAXOSMITHKLINE Translated by MAPI RESEARCH INSTITUTE Senior Translator: Ray Kirk

(C) The Asthma Control Questionnaire is copyrighted. It may not be altered, sold (paper or electronic), translated or adapted for another medium without the permission of Elizabeth Juniper.

NOVEMBER 2001 
(ENGLISH VERSION FOR NEW ZEALAND) DATE:

\title{
INSTRUCTIONS
}

Please answer questions 1 - 6 .

Please circle the number of the response that best describes how you have been during the past week.

1. In general, during the past week how often were you woken by your asthma during the night?

0 Never

1 Hardly ever

2 A few times

3 Several times

4 Many times

5 A great many times

6 Unable to sleep because of asthma

2. In general, during the past week how uncomfortable were your asthma symptoms when you woke up in the morning?

0 No symptoms

1 Very mild symptoms

2 Mild symptoms

3 Moderate symptoms

4 Quite severe symptoms

5 Severe symptoms

6 Very severe symptoms

3. In general, during the past week, how limited were you in your activities because of your asthma?

0 Not limited at all

1 Very slightly limited

2 Slightly limited

3 Moderately limited

4 Very limited

5 Extremely limited

6 Totally limited

4. In general, during the past week, how much shortness of breath did you experience because of your asthma?

\author{
0 None \\ 1 A very little \\ 2 A little \\ 3 A moderate amount \\ 4 Quite a lot \\ 5 A great deal \\ 6 A very great deal
}


5. In general, during the past week, how much time did you wheeze?
0 Never
1 Hardly any of the time
2 A little of the time
3 A moderate amount of the time
4 A lot of the time
5 Most of the time
6 All the time

6. On average, during the past week, 0 None how many puffs/inhalations of your reliever (eg. Ventolin/Bricanyl) have you used each day? (If you are not sure how to answer this question, please ask for help)

11 - 2 puffs/inhalations most days

23 - 4 puffs/inhalations most days

$35-8$ puffs/inhalations most days

49 - 12 puffs/inhalations most days

$513-16$ puffs/inhalations most days

6 More than 16 puffs/inhalations most days

\section{To be completed by a member of the clinic staff}

7. $\mathrm{FEV}_{1}$ pre-bronchodilator:
$0>95 \%$ predicted
$195-90 \%$
$289-80 \%$
$379-70 \%$
$469-60 \%$
$5 \quad 59-50 \%$
$6<50 \%$ predicted

$\mathrm{FEV}_{1}$ predicted:

FEV $1 \%$ predicted:

(Record actual values on the dotted

lines and score the $\mathrm{FEV}_{1} \%$ predicted

in the next column) 


\section{Appendix XI: Asthma Quality of Life}

Questionnaire with Standardised Activities

$$
\text { (AQLQ-S) }
$$




\title{
ASTHMA QUALITY OF LIFE QUESTIONNAIRE WITH STANDARDISED ACTIVITIES (AQLQ(S))
}

\author{
SELF-ADMINISTERED \\ NEW ZEALAND VERSION
}

(C) ORIGINAL 1997

(C) REVISION 1996

QOL TECHNOLOGIES LTD
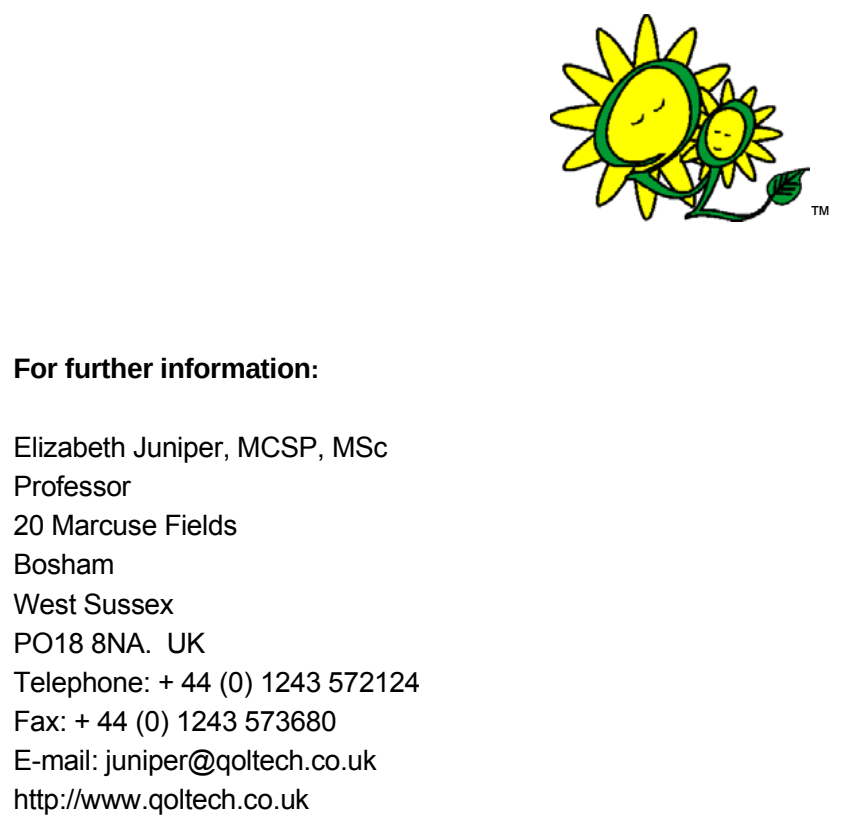

The AQLQ is copyrighted. It may not be altered, sold (paper or electronic), (C) translated or adapted for another medium without the permission of Elizabeth Juniper. 
ASTHMA QUALITY OF LIFE QUESTIONNAIRE (S)

(NEW ZEALAND VERSION)

SELF-ADMINISTERED
PATIENT ID

DATE

Page 1 of 5

Please complete all the questions by circling the number that best describes how you have been during the last 2 weeks as a result of your asthma.

HOW LIMITED HAVE YOU BEEN DURING THE LAST 2 WEEKS IN THESE ACTIVITIES AS A RESULT OF YOUR ASTHMA?

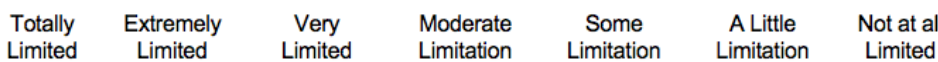

1. STRENUOUS ACTIVITIES

(such as hurrying, exercising,

running up stairs, sports)

2. MODERATE ACTIVITIES

(such as walking, housework, gardening, shopping, climbing

12

23

34

4

5

$\begin{array}{ll}6 & 7\end{array}$ stairs)

3. SOCIAL ACTIVITIES

such as talking, playing with

pets/children, visiting

friends/relatives)

4. WORK-RELATED

ACTIVITIES* (tasks you

have to do at work)

*If your are not employed or self-employed, these should be tasks you have to do most days.
5. SLEEPING
12
3
4
5
$6 \quad 7$

HOW MUCH DISCOMFORT OR DISTRESS HAVE YOU FELT DURING THE LAST 2 WEEKS?

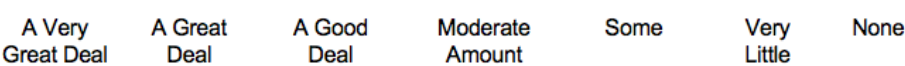

6. How much discomfort or distress have you felt over the last 2 weeks as a result of CHEST TIGHTNESS?

2

3

4

56

7 
IN GENERAL, HOW MUCH OF THE TIME DURING THE LAST 2 WEEKS DID YOU:

\begin{tabular}{|c|c|c|c|c|c|}
\hline $\begin{array}{l}\text { All of the } \\
\text { Time }\end{array}$ & $\begin{array}{c}\text { Most of } \\
\text { the Time }\end{array}$ & $\begin{array}{l}\text { A Good } \\
\text { Bit of the } \\
\text { Time }\end{array}$ & $\begin{array}{l}\text { Some of } \\
\text { the Time }\end{array}$ & $\begin{array}{c}\text { A Little } \\
\text { of the } \\
\text { Time }\end{array}$ & $\begin{array}{l}\text { Hardly Any } \\
\text { of the Time }\end{array}$ \\
\hline
\end{tabular}

7. Feel CONCERNED ABOUT HAVING ASTHMA?

$\begin{array}{llll}1 & 2 & 3 & 4\end{array}$

$5-6$

7

8. Feel SHORT OF BREATH as a result of your asthma?

$\begin{array}{lllllll}1 & 2 & 3 & 4 & 5 & 6 & 7\end{array}$

9. Experience asthma symptoms as a RESULT OF BEING EXPOSED TO CIGARETTE SMOKE?

10. Experience a WHEEZE in your chest?

$\begin{array}{lllllll}1 & 2 & 3 & 4 & 5 & 6 & 7\end{array}$

11. Feel you had to AVOID A SITUATION OR ENVIRONMENT BECAUSE OF CIGARETTE SMOKE?

HOW MUCH DISCOMFORT OR DISTRESS HAVE YOU FELT DURING THE LAST 2 WEEKS?

12. How much discomfort or distress have you felt over the last 2 weeks as a result $\begin{array}{cccccc}\text { A Very } & \text { A Great } & \text { A Good } & \text { Moderate } & \text { Some } & \text { Very } \\ \text { Great Deal } & \text { Deal } & \text { Deal } & \text { Amount } & & \text { Little }\end{array}$ of COUGHING?

IN GENERAL, HOW MUCH OF THE TIME DURING THE LAST 2 WEEKS DID YOU:

\begin{tabular}{|c|c|c|c|c|c|c|}
\hline $\begin{array}{l}\text { All of the } \\
\text { Time }\end{array}$ & $\begin{array}{l}\text { Most of } \\
\text { the Time }\end{array}$ & $\begin{array}{l}\text { A Good Bit } \\
\text { of the } \\
\text { Time }\end{array}$ & $\begin{array}{l}\text { Some of } \\
\text { the Time }\end{array}$ & $\begin{array}{l}\text { A Little of } \\
\text { the Time }\end{array}$ & $\begin{array}{l}\text { Hardly Any } \\
\text { of the Time }\end{array}$ & $\begin{array}{c}\text { None of the } \\
\text { Time }\end{array}$ \\
\hline
\end{tabular}

13. Feel FRUSTRATED as a result of your asthma?

$\begin{array}{lllllll}1 & 2 & 3 & 4 & 5 & 6 & 7\end{array}$

14. Experience a feeling of CHEST HEAVINESS?

$\begin{array}{lllllll}1 & 2 & 3 & 4 & 5 & 6 & 7\end{array}$


IN GENERAL, HOW MUCH OF THE TIME DURING THE LAST 2 WEEKS DID YOU:

15. Feel CONCERNED ABOUT THE NEED TO USE MEDICATION for your asthma?

16. Feel the need to CLEAR YOUR THROAT?

$$
\begin{aligned}
& \text { All of the } \\
& \text { Time }
\end{aligned}
$$

23

4

5

6

7

. Experience asthma symptoms as a RESULT OF BEING EXPOSED TO DUST?

18. Experience DIFFICULTY BREATHING OUT as a result of your asthma?

$\begin{array}{lllllll}1 & 2 & 3 & 4 & 5 & 6 & 7\end{array}$

$\begin{array}{llllllll}1 & 2 & 3 & 4 & 5 & 6 & 7\end{array}$

9. Feel you had to AVOID A SITUATION OR ENVIRONMENT BECAUSE OF DUST?

20. WAKE UP IN THE MORNING WITH ASTHMA SYMPTOMS?

1

2

3

4

5

$6 \quad 7$

21. Feel AFRAID OF NOT HAVING YOUR ASTHMA MEDICATION AVAILABLE?

22. Feel bothered by HEAVY BREATHING?

23. Experience asthma symptoms as a RESULT OF THE WEATHER OR AIR POLLUTION/POLLEN OUTSIDE?

24. Were you WOKEN AT NIGHT by your asthma?

25. AVOID OR LIMIT GOING OUTSIDE BECAUSE OF THE WEATHER OR AIR POLLUTION/POLLEN?

$\begin{array}{lllllll}1 & 2 & 3 & 4 & 5 & 6 & 7 \\ 1 & 2 & 3 & 4 & 5 & 6 & 7 \\ 1 & 2 & 3 & 4 & 5 & 6 & 7 \\ 1 & 2 & 3 & 4 & 5 & 6 & 7 \\ 1 & 2 & 3 & 4 & 5 & 6 & 7 \\ 1 & 2 & 3 & 4 & 5 & 6 & 7\end{array}$


IN GENERAL, HOW MUCH OF THE TIME DURING THE LAST 2 WEEKS DID YOU:

All of the Most of the A Good Bit Some of Time Time of the Time the Time

A Little of the Hardly Any None of Time of the Time the Time

26. Experience asthma symptoms as a RESULT OF BEING EXPOSED TO STRONG SMELLS OR PERFUME?

27. Feel AFRAID OF GETTING OUT OF BREATH?

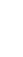

$\begin{array}{lllllll}1 & 2 & 3 & 4 & 5 & 6 & 7\end{array}$

$\begin{array}{lllllll}1 & 2 & 3 & 4 & 5 & 6 & 7\end{array}$

28. Feel you had to AVOID A SITUATION OR ENVIRONMENT BECAUSE OF STRONG SMELLS OR PERFUME?

29. Has you asthma INTERFERED WITH GETTING A GOOD NIGHT'S SLEEP?

30. Have the feeling of FIGHTING FOR BREATH?

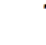

1

2

3

$$
4
$$

5

6

7

1

1

1

3

4

5

6

7

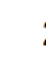

HOW LIMITED HAVE YOU BEEN DURING THE LAST 2 WEEKS?

\section{Most Not}

Several

Not Done

1 OF ACTIVITIES that you would have liked to have done during the last 2 weeks? How much has your range of activities been limited by your asthma? 


\section{Appendix XII: Participant Information}

\section{Sheet for Study Five}

Participant Information Sheet

Study title: Change in serum periostin level in severe exacerbations of asthma (PER03)

Locality: Medical Research Institute of Ethics

13/NTB/191

New Zealand

committee ref.:

Lead

Prof. Richard Beasley

Contact phone 048050147

investigator: number:

You are invited to take part in a study to determine the change in periostin levels over the twelve weeks following an exacerbation of asthma in a group of patients, aged 1875 years. Whether or not you take part is your choice. If you don't want to take part, you don't have to give a reason, and it won't affect the care you receive. If you do want to take part now, but change your mind later, you can pull out of the study at any time.

You will not be eligible to take part in this study if any of the following applies to you:

- You are known to be pregnant

- You have been admitted to hospital (not including admissions for asthma) or had surgery in the last 3 months

- You have had a bone fracture in the last 3 months

This Participant Information Sheet will help you decide if you'd like to take part. It sets out why we are doing the study, what your participation would involve, what the benefits and risks to you might be, and what would happen after the study ends. We 
will go through this information with you and answer any questions you may have. We expect this will take about 20 minutes. You may also want to talk about the study with other people, such as family, whānau, friends, or healthcare providers. Feel free to do this.

If you agree to take part in this study, you will be asked to sign the Consent Form on the last page of this document. You will be given a copy of both the Participant Information Sheet and the Consent Form to keep.

This document is 8 pages long, including the Consent Form. Please make sure you have read all the pages. If you require an interpreter, this will be arranged.

\section{Why are we doing the study?}

The protein periostin has been found to be present in a number of medical conditions, including asthma, early bone healing, progression of various cancers and kidney disease related to high blood pressure.

It is easily measured (by a blood test) and in the future it may be used to determine the status of a person's disease, including its severity, if it is progressing or if it is no longer present. Proteins like periostin that can allow doctors to assess a disease are known as biomarkers. This study is being conducted to determine changes in periostin levels over time in patients with asthma which will allow this disease to be better monitored and treated in the future.

We aim to have a total of 34 participants involved in this study.

The Sponsor of this study is the Medical Research Institute of New Zealand. The study is being funded by Genentech Inc, a member of the Roche Group.

The study has been approved by the Northern B Health and Disability Ethics Committee, reference number 13/NTB/191. 


\section{What would your participation involve?}

The study is being conducted at the Medical Research Institute of New Zealand, based in Wellington Hospital. If applicable study visits may also take place at your GP surgery, local hospital, or at your home, with your permission. If you agree to be a part of the study you will complete your first visit within 24 hours of your exacerbation of asthma and then attend for a further 5 visits ( 6 in total). Visits 1 and 6 will take about an hour and visits 2-5 will take about half an hour.

\section{Visit Outline}

\section{Visit 1}

After reading this information sheet and signing the informed consent form, the study investigator will provide you with a general health questionnaire which you will fill out in their presence. The study investigator will then discuss your medical history and current medications so that a full picture is gained of your current health. If required we will contact your GP (with your permission) to clarify aspects of your medical history or current medications. You will be given two asthma questionnaires (AQLQ and ACQ-5) which you will fill out in the presence of the study investigator.

The following tests will then be carried out.

- We will measure nitric oxide (a gas that you normally breathe out) by asking you to breathe into a tube.

- To check the health and function of your lungs you will be asked to do some breathing exercises. You will then follow the instructions given to you by the investigator, for a few minutes. This test will be done so that 3 of your best measurements are recorded. This may take up to 8 measurements (or more if you agree) in total, so that we can record the best results.

- We will take a small blood sample, to measure your full blood count, electrolytes and creatinine as well as serum IgE, and serum periostin level.

- Finally we will take a nasal swab, which will be inserted into your nose and a sample will be collected and analysed for viruses. 


\section{Visit 2-5}

You will be asked about your current health and any changes to your health since your last visit. We will then ask you to perform the following:

- We will measure nitric oxide (a gas that you normally breathe out) by asking you to breathe into a tube.

- To check the health and function of your lungs you will be asked to do some breathing exercises. You will then follow the instructions given to you by the investigator, for a few minutes. This test will be done so that 3 of your best measurements are recorded. This may take up to 8 measurements (or more if you agree) in total, so that we can record the best results.

- We will take a small blood sample, to measure your full blood count, electrolytes and creatinine as well as serum IgE, and serum periostin level.

\section{Visit 6}

You will be asked about your current health and any changes to your health since your last visit. You will complete both of the asthma questionnaires again in the presence of the study investigator. The following tests will again be carried out:

- We will measure nitric oxide (a gas that you normally breathe out) by asking you to breathe into a tube.

- To check the health and function of your lungs you will be asked to do some breathing exercises. You will then follow the instructions given to you by the investigator, for a few minutes. This test will be done so that 3 of your best measurements are recorded. This may take up to 8 measurements (or more if you agree) in total, so that we can record the best results.

- We will take a small blood sample, to measure your full blood count, electrolytes and creatinine as well as serum IgE, and serum periostin level.

\section{What are the possible benefits and risks to you of participating?}

\section{Benefits}


There will be no direct benefit to you in participating in this study, you will however be part of research which may benefit yourself and others in the future.

\section{Study Procedure Risks/ Side Effects}

\section{Blood Samples}

You may experience some discomfort during the taking of a blood sample and there is always the risk of bleeding, swelling and bruising at the site of the needle during sampling. All samples will be taken by trained staff. Around $10 \mathrm{ml}$ of blood will be taken during the study. In some cases we may require extra samples, for example to re-do a test that could not be analysed.

A local laboratory will analyse your full blood count, as well as serum IgE.

Periostin will be analysed at a central laboratory outside of New Zealand, by Genentech. This laboratory is:

Covance Central Laboratory Services

8211 Scicor Drive

Indianapolis, Indiana 46214

USA

Your blood samples will be coded with a unique subject number and your name will not be used to identify the samples. The samples analysed locally will be destroyed by the laboratory once the results are reported. The samples sent to Genentech may be stored for 5 years after the study. You may hold beliefs about a sacred and shared value of all or any tissue samples removed. The cultural issues associated with sending your samples overseas and/or storing your tissue and/or undertaking genetic analysis on them should be discussed with your family/whānau as appropriate. There are a range of views held by Māori around these issues; some iwi disagree with storage of samples and genetic testing citing whakapapa and advise their people to consult prior to participation in research where this occurs. However it is acknowledged that individuals have the right to choose.

\section{Breathing Exercises}


You may feel some discomfort during or after performing the breathing exercises, however this will be transient and you will be monitored constantly throughout the tests by trained technicians/ study investigators. You will be seated at all times for the tests.

\section{Nasal Swab sample}

You may feel some discomfort when the nasal swab is inserted to collect the sample. The sample will be collected at the Institute and then sent to Genentech (outside of New Zealand) for analysis of respiratory viruses. After measurement, the sample will be destroyed

\section{Cost}

There will be no cost to you to take part in this study and you will be compensated for your time and travel expenses to attend your study visits ( $\$ 20$ for visits $2-5$ and $\$ 45$ for visit 6).

\section{General Health Care}

Your general health care remains with your GP throughout the time you are in the study and if you experience any problems you should contact your usual health care provider. As part of the informed consent process we will ask for your permission to inform your GP of any unexpected clinically significant findings.

As part of the study you will be seen by study investigators, study doctors and trained staff who will conduct all the study specific assessments.

\section{What would happen if you were injured in the study?}

If you were injured as a result of treatment given as part of this study, which is unlikely, you won't be eligible for compensation from ACC. However, compensation would be available from the study's sponsor, (Medical Research Institute of New Zealand), in line with current guidelines. We can give you a copy of these guidelines if you wish. You would be able to take action through the courts if you disagreed with the amount of compensation provided. 
If you have private health or life insurance, you may wish to check with your insurer that taking part in this study won't affect your cover.

\section{What are the rights of participants in the study?}

Your participation is entirely voluntary (your choice). You do not have to take part in this study, and if you choose not to take part it will in no way affect your future health care. If you do agree to take part you are free to withdraw from the study at any time, without having to give a reason.

\section{Confidentiality and Data Privacy}

If you decide to participate, the study doctor and relevant Institute staff will collect medical and personal information about you, as part of doing the study. By agreeing to take part in this research, you will allow your medical information and results to be seen by people who check that the research was done properly, for example the ethics committee and study monitor.Nothing which could personally identify you will be used in any reports on this study, or provided as part of future studies. Your personal information (for example name, sex, age and medical conditions) and other information will be identified by a study number (i.e. coded). The study records will be stored securely during the course of the study and once it is completed. After all participants have completed the study and the data has been monitored, the records will be archived for a minimum of 10 years as per New Zealand regulations. The records will then be securely destroyed.

\section{What will happen after the study ends, or if you pull out?}

Once the study has finished and the data has been analysed you will be able to find out the results from the study. You may also request a copy of your individual lung function results.

The sponsor may stop the study at any time. You may be asked to leave the trial, for the following reasons: 
- In the Investigator's opinion it would not be in your best interest to continue in the study

- Any safety concerns

- You do not follow instructions during the study visits

Where can you go for more information about the study, or to raise concerns or complaints?

If you have any questions, concerns or complaints about the study at any stage, you can contact the study investigator:

Name: $\quad$ Dr Ruth Semprini

Phone: $\quad$ (04) 8050232

E-mail: $\quad$ ruth.semprini@mrinz.ac.nz

For Maori health support, please contact:

Whānau Care Services, Cultural Care Centre, Level 2, Wellington Hospital

Phone: $\quad 0800999442$ or 048060948

Email: $\quad$ wcs@ccdhb.org.nz

If you want to talk to someone who isn't involved with the study, you can contact an independent health and disability advocate on:

Phone: $\quad 0800555050$

Fax: $\quad 08002$ SUPPORT (0800 2787 7678)

Email: $\quad$ advocacy@hdc.org.nz

You can also contact the health and disability ethics committee (HDEC) that approved this study on:

Phone: $\quad 08004$ ETHICS (0800 4384 427)

Email: $\quad$ hdecs@moh.govt.nz 


\section{Consent Form}

Study title: Change in serum periostin level in severe exacerbations of asthma (PER03)

Participant Number:

Please tick to indicate you consent to the following:

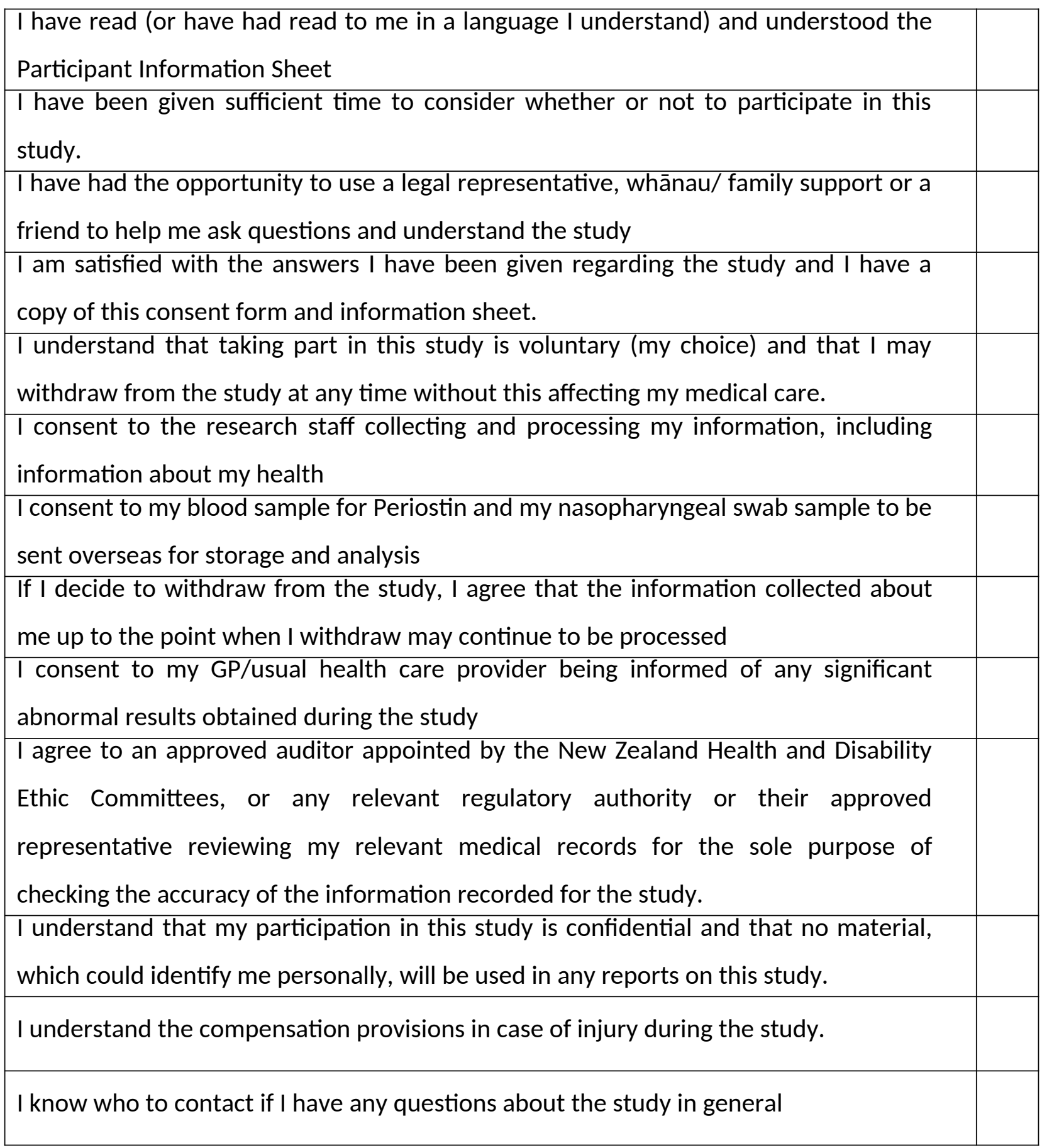


I understand my responsibilities as a study participant.

\begin{tabular}{|l|l|l|}
\hline \multicolumn{2}{|c|}{ Yes } & \\
\hline I wish to receive a summary of the results from the study. & & \\
\hline $\begin{array}{l}\text { I give consent for study investigators to contact my GP/ usual health care } \\
\text { provider to clarify my medical history or current medications, if required }\end{array}$ & & \\
\hline $\begin{array}{l}\text { I give consent for study investigators to inform my GP/ usual health care } \\
\text { provider of my participation in this study }\end{array}$ & & \\
\hline
\end{tabular}

Declaration by participant:

I hereby consent to take part in this study

Participant name (print)

Participant signature

Date

\section{Declaration by member of research team:}

I have given a verbal explanation of the research project to the participant, and have answered the participant's questions about it.

I believe that the participant understands the study and has given informed consent to participate.

$\overline{\text { Name of person conducting informed consent discussion }}$

(print)

Signature of person conducting informed consent Date

discussion 


\section{Appendix XIII: Protocol for Study Five}

Protocol

Change in serum periostin level in severe exacerbations of asthma

Protocol No.: PERO3

UTN: U1111-1150-0474

ANZCTRN: ACTRN12614000443695

\section{Rationale}

Periostin is an extracellular matrix protein encoded by the POSTN gene (an IL-4/ IL-13 inducible gene) thought to be an adhesion molecule because it possesses four fasciclin I domains. ${ }^{16}$ Periostin has been shown to be secreted in the extracellular matrix from the basolateral surface of bronchial epithelial cells after stimulation with IL-13. ${ }^{98}$

Periostin has been studied in patients with asthma, expression levels of the POSTN gene corresponding to reticular basement membrane thickness an indicator of subepithelial fibrosis. ${ }^{98}$ Periostin is thought to enhance fibrosis in the airways by binding to other extracellular proteins collagen, fibronectin and tenascin C. ${ }^{239}$ Periostin may also have a role in other $\mathrm{TH} 2$ driven inflammation such as nasal polyps, eosinophilic oesophagitis, allergic rhinitis and atopic dermatitis.

A highly specific serum assay for periostin has been produced which allows levels of periostin in peripheral blood to be tested. Through use of this assay periostin has been identified as a systemic biomarker for airway eosinophilia. Periostin levels in peripheral blood correspond to eosinophilic inflammation in tissue taken from the airways of patients with asthma. ${ }^{98}$ Using periostin as a systemic biomarker of airway eosinophilia would provide a minimally invasive monitoring technique which could be used to monitor severity, response to treatment and determine treatment options for patients with asthma. ${ }^{98}$ In order to use serum periostin levels as a biomarker in 
asthma a more complete picture of periostin production in asthma needs to be formed. This study of the periostin levels in severe exacerbations of asthma would provide further information on the production of periostin in asthma. Until now the periostin assay has only been used to determine the relationship of periostin with eosinophilic airway inflammation in patients not responding to inhaled steroid treatment and has not been studied in patients with acute severe exacerbations of asthma. ${ }^{241}$

This study has been initiated and designed by the Medical Research Institute of New Zealand in collaboration with Genentech, Inc (a member of the Roche group). The Institute is the Sponsor of this study and undertakes Sponsor responsibilities for the purposes of ethical and any applicable regulatory requirements. Genentech are fully funding the study.

\section{Objective}

To determine the change in serum periostin level over the 12 weeks following a severe exacerbation of asthma which resulted in hospital admission

\section{Outcomes}

\section{Primary outcome measure:}

Changes in periostin level over the 12 weeks following a severe exacerbation of asthma

\section{Secondary outcome measures:}

Investigation of potential associations between serum periostin and the following:

- $\quad A Q L Q$ score (from respiratory health questionnaire)

- Non-asthmatic health conditions (as captured by general health questionnaire e.g. diabetes)

- Spirometry (FEV1 and FVC)

- Fractional exhaled Nitric Oxide (FeNO) level

- Full blood count including white cell differential

- Serum IgE

\section{Exploratory outcome measures (RCR only)}


- Other immune mediated biomarkers

- RNA- based biomarker analyses

\section{Study design}

\section{Participants:}

34 Participants will be recruited within 24 hours of commencing systemic corticosteroids for a severe exacerbation of asthma.

\section{Inclusion Criteria}

- $\quad$ Aged between 18 to 75 years

- Able to provide written informed consent

- Doctor diagnosis of asthma

- Exacerbation of asthma, defined as one or both of the following criteria:

- Use of systemic corticosteroids (tablets, suspension, or injection), or an increase from a stable maintenance dose, for at least 3 days. For consistency, courses of corticosteroids separated by 1 week or more should be treated as separate severe exacerbations.

- A hospitalization or Emergency Department visit because of asthma, requiring systemic corticosteroids.

- Participants prescribed a dose of systemic corticosteroids for at least 3 days will be eligible for the study, though it is recognised they will not have taken systemic corticosteroids for 3 days at the point of enrolment. Participants will not be eligible if they have commenced systemic corticosteroids greater than 24 hours prior to enrolment.

- Participants who have increased from their stable maintenance dose of systemic corticosteroids will only be eligible if this dose increase is confirmed to be less than 24 hours prior to enrolment.

\section{Exclusion Criteria}

- Known Pregnancy*

- Significant co morbidities (determined at investigator discretion) 
- Hospital admission within last 3 months for condition other than asthma

- Surgery within last 3 months, determined at investigator discretion (this includes all major surgery requiring general anaesthetic, dental extractions and root canal procedures. This does not include minor procedures, including but not limited to mole or wart removal, dental fillings etc).

- Bone fracture within the last 3 months

- Any other safety concern at the investigator's discretion

* If a potential subject is aware they are currently pregnant, or actively trying to conceive, they will be excluded from the study as pregnancy can affect FeNO, eosinophil and spirometry results. No pregnancy test will be performed on women of childbearing potential as there are no safety concerns relating to this study.

Following screening, 34 participants will be enrolled.

\section{Study Procedures}

\section{Study Visits}

Participants will be seen 6 times. The location of this visit will depend upon the circumstances of the potential participant's asthma exacerbation, but will be within a pre- approved Locality as per HDEC guidelines. Visit 1 will be within 24 hours of starting systemic corticosteroids (or from increasing from a stable maintenance dose of systemic corticosteroids) and then participants will be seen one, two, four, eight and 12 weeks post Visit 1.

For visits 2-6 there will be a visit window of +/- 3 days. If a visit is delayed the calculated schedule of visit dates from baseline (visit 1) shall be maintained. Any delays greater than +/- 3 days will be recorded as protocol deviations, and included in the final analysis.

\section{Visit 1}

Details of the severe asthma exacerbation documented as follows: 
- Written Informed Consent

- Completion of General Health Questionnaire

- Completion of Respiratory Health Questionnaire AQLQ

- Completion of ACQ5 Questionnaire

- Measurement of Body Mass Index (BMI)

- Spirometry assessment (Measurement of FEV ${ }_{1}$ and FVC)

- Measurement of FeNO

- Nasopharyngeal swab for viral PCR

- Laboratory samples (taken within 24 hours of participant commencing or increasing maintenance systemic corticosteroids) for measurement of:

- Full blood count (including differential white cell count)

- Serum IgE

- Serum periostin level.

- Serum and RNA- based biomarker analyses will also be conducted on samples collected for the Roche Clinical Repository (RCR). These samples will only be collected from participants who provide consent on the RCR informed consent form which is a separate consent to the main study (see Optional samples for Roche Clinical Repository).

\section{Visit 2-5}

- Spirometry assessment (Measurement of FEV ${ }_{1}$ and FVC)

- Measurement of FeNO

- Laboratory samples for measurement of:

- Periostin level

- Full blood count (including differential white cell count)

- Serum IgE

- Serum periostin level

- Serum and RNA based biomarker analyses will also be conducted on samples collected for the Roche Clinical Repository (RCR). These samples will only be collected from participants who provide consent on the RCR informed consent form which is a separate consent to the main study (see Optional samples for Roche Clinical Repository). 


\section{Visit 6}

At 12 weeks participants will attend an end of study visit during which the following will be performed:

- Respiratory Health Questionnaire AQLQ

- ACQ5 Questionnaire

- Spirometry (FEV ${ }_{1}$ and FVC)

- $\mathrm{FeNO}$

- Laboratory samples for measurement of:

- Full blood count (including differential white cell count)

- Serum IgE

- Serum periostin level

- Serum and RNA based biomarker analyses will also be conducted on samples collected for the Roche Clinical Repository (RCR). These samples will only be collected from participants who provide consent on the RCR informed consent form which is a separate consent to the main study (see Optional samples for Roche Clinical Repository).

\section{Informed Consent}

Informed consent will be obtained according to ethical and GCP guidelines, and will take place in an appropriate environment. Each participant will sign an informed consent form at the first visit, prior to conducting the procedures listed above.

\section{Respiratory Health Questionnaire}

The Asthma Quality of Life Questionnaire with Standardised Activities (AQLQ(S)) is a validated 32 question self administered questionnaire that will be used to obtain severity of asthma in the two weeks prior to visit 1 and 6.

The Asthma Control Questionnaire (ACQ5) is a validated 5 question self administered questionnaire that will be used to obtain severity of asthma in the 1 week prior to visit 1 and 6. 


\section{General Health Questionnaire}

The health questionnaire will be used to obtain the current health status of participants, including a history of cancer, cardiovascular disease, gastro-oesophageal reflux (GORD), rhinitis, sinusitis, and eczema (see appendix 1). As part of the assessment of general health participants will undergo height and weight measurement to obtain their BMI.

\section{Lung Function Tests}

Nitric Oxide

Nitric oxide (FeNO) will be measured using a nitric oxide monitor (NIOX MINO; Aerocrine AB, Solna, Sweden) according to the ATS guidelines published in 2005. Seated subjects will exhale fully and then inhale ambient air through a nitric oxide scrubber to total lung capacity. Subjects will then exhale against an automatically adjusting resistance to achieve a constant exhalation flow rate of $50 \mathrm{ml} / \mathrm{s}+/-\mathrm{mls}$. Resistance will be adjusted so that an upper airway pressure of $10-20 \mathrm{~cm} \mathrm{H} 2 \mathrm{O}$ is maintained throughout exhalation, sufficient to close the velum and exclude nasal air. FeNO measurements will be taken from a stable plateau in exhaled nitric oxide concentration during an exhalation. Exhalations where flow rate and plateau criteria are not met will be deemed not acceptable for measurement. One acceptable exhalation will be measured and recorded at each time point. Measurements will be made before other pulmonary function testing. Participants will be tested within 24 hours of a hospital attendance or commencement of systemic steroids, times of last consumption of caffeine, food and tobacco prior to testing will be documented.

\section{Spirometry}

Participants will undergo pulmonary testing using a spirometer which meets the guidelines outlined by the American Thoracic Society 2005-Standardisation of spirometry. A portable spirometer (Micro Medical Micro DL) attached to a laptop so that flow loops can be viewed at time of procedure.

Last use of bronchodilator medication (inhaler, nebuliser) prior to testing will be documented. 


\section{General guidelines}

All tests will be performed:

Sitting

With nose clips on

The tests will be clearly explained to the subject before they are started and positive encouragement given at all stages of the test.

\section{Acceptability criteria}

Criteria for technically unsatisfactory tests:

- Coughing during the first second of the exhalation

- Glottis closure that influences the measurement

- Effort that is not maximal throughout

- Obstructed mouthpiece e.g. tongue in front of the mouthpiece

- A leak in the system or around the mouthpiece

- Excessive hesitation at the start of expiration (extrapolated volume $<5 \%$ or $150 \mathrm{ml}$ of FVC -whichever is greater.

- Early termination of test by subject

\section{Repeatability criteria}

- Less than $150 \mathrm{ml}$ variation in FEV1 between attempts

- Less than $150 \mathrm{ml}$ variation in FVC between attempts

Testing should be continued until 3 acceptable manoeuvres have been completed or the subject has performed 8 manoeuvres-more can be done if the subject agrees. According to ATS guidelines subjects who are unable to produce reproducible flow volume loops (reproducible flow loops are those with $<150 \mathrm{ml}$ variation in FEV1 and FVC) will not be excluded from analysis. In subjects who were not able to produce 3 acceptable flow volume loops comments regarding the technical acceptability of their testing will be made.

\section{Laboratory Samples}


Samples for the following tests will be analysed by a local laboratory:

- Serum IgE

- Full blood count (including differential white cell count)

Samples will be analysed as per the laboratory's standard operating procedures.

Samples for the following tests will be sent to Genentech (or designee) to be analysed:

- Serum periostin level

- Optional serum and RNA- based biomarker analyses (as per the RCR analysis below)

- Nasopharyngeal swab for viral PCR

\section{Optional Samples for Roche Clinical Repository (RCR)}

\section{Overview of the Roche Clinical Repository}

The Roche Clinical Repository (RCR) is a centrally administered group of facilities for the long-term storage of human biologic specimens, including body fluids, solid tissues, and derivatives thereof (e.g., DNA, RNA, proteins, peptides). The collection and analysis of RCR specimens will facilitate the rational design of new pharmaceutical agents and the development of diagnostic tests, which may allow for individualized drug therapy for patients in the future. Specimens for the RCR will be collected from patients who give specific consent to participate in this optional research.

\section{Approval by the Institutional Review Board or Ethics Committee}

Sampling for the RCR is contingent upon the review and approval of the exploratory research and the RCR specific Informed Consent Form (ICF) by the New Zealand Health and Disability Ethics Committees.

\section{Optional RCR Sample Collection}

For sampling procedures, storage conditions, and shipment instructions, see the laboratory manual. RCR specimens will be destroyed no later than 15 years after the date of final closure of the associated clinical database. The RCR storage period will be in accordance with the IRB/EC-approved ICF and applicable laws (e.g., health authority 
requirements). The dynamic biomarker specimens will be subject to confidentiality standards, i.e. by coding each participant enrolled in the study through assignment of a unique subject identification number. This means that participant names are not included in data sets that are transmitted to any location outside of the MRINZ.

\section{Confidentiality}

Data generated from RCR specimens must be available for inspection upon request by representatives of national and local health authorities, and Roche monitors, representatives, and collaborators, as appropriate. Participant medical information associated with RCR specimens is confidential and may only be disclosed to third parties as permitted by the ICF (or separate authorization for use and disclosure of personal health information) signed by the participant, unless permitted or required by law. Data derived from RCR specimen analysis on individual participants will generally not be provided to study investigators unless a request for research use is granted. The aggregate results of any research conducted using RCR specimens will be available in accordance with the effective Roche policy on study data publication.

Any inventions and resulting patents, improvements, and/or know-how originating from the use of the RCR data will become and remain the exclusive and unburdened property of Roche, except where agreed otherwise.

\section{Consent to Participate in the Roche Clinical Repository}

There will be a separate ICF which will contain information that addresses participation in the RCR. The investigator or authorized designee will explain to each participant the objectives, methods, and potential hazards of participation in the RCR. Participants will be told that they are free to refuse to participate and may withdraw their specimens at any time and for any reason during the storage period. A separate, specific signature will be required to document a participant's agreement to provide optional RCR specimens. Participants who decline to participate will not provide a separate signature. In the event of an RCR participant's death or loss of competence, the participant's specimens and data will continue to be used as part of the RCR research.

\section{Withdrawal from the Roche Clinical Repository}


Participants who give consent to provide RCR specimens have the right to withdraw their specimens from the RCR at any time for any reason. If a participant wishes to withdraw consent to the testing of his or her specimens, the investigator will inform the Genentech in writing of the participant's wishes. The participant will be provided with instructions on how to withdraw consent after the trial is closed. A participant's withdrawal from this study does not, by itself, constitute withdrawal of specimens from the RCR. Likewise, a participant's withdrawal from the RCR does not constitute withdrawal from this study.

\section{Monitoring and Oversight}

RCR specimens will be tracked in a manner consistent with Good Clinical Practice (GCP) by a quality-controlled, auditable, and appropriately validated laboratory information management system, to ensure compliance with data confidentiality as well as adherence to authorized use of specimens as specified in this protocol and in the ICF. Roche and MRINZ monitors and auditors will have direct access to appropriate parts of records relating to patient participation in the RCR for the purposes of verifying the data provided to Roche, as necessary. The site will permit monitoring, audits, IRB/EC review, and health authority inspections by providing direct access to source data and documents related to the RCR samples.

\section{Safety Monitoring}

\section{Adverse Event Reporting}

For the purposes of this study, the study duration is considered to start once the informed consent form has been signed by the participant. The study period will be deemed to have ended when the study participant completes their final study visit (visit 6) and leaves MRINZ premises. The MRINZ will be responsible for reviewing the blood tests taken during the study visits and reporting any clinically significant abnormalities to the participant's GP/ primary healthcare provider, as necessary.

\section{Adverse Events}


An adverse event is any untoward medical occurrence in a study subject temporally associated with the testing as part of the study. An adverse event can therefore be any unfavourable and unintended sign, symptom or disease temporally associated with the testing carried out in the study. Adverse event data will be collected by investigators during the study visits and after reviewing the test results.

\section{Serious Adverse Events (SAEs)}

For the purposes of this study the following events will be considered to be SAEs:

- Death

- Life-threatening event

- Permanently disabling or incapacitating event

- Hospitalisation. Hospitalisation for the purposes of SAE reporting is defined as an admission to hospital and does not include a presentation to the Emergency Department followed by discharge without admission or an admission for elective reasons

- Any other significant medical event considered serious by the study investigator

All SAEs will be reported to the NZ HDEC and regulatory authorities as per current guidelines. SAEs and AEs will be followed up until resolution, or judged to be permanent. All SAEs will be recorded by the MRINZ (as study sponsor) within 24 hours of the study investigators becoming aware of the event.

\section{Abnormal Laboratory Values}

Not every laboratory abnormality qualifies as an adverse event. A laboratory test result should be reported as an adverse event if it meets any of the following criteria:

- Accompanied by clinical symptoms

- Results in a medical intervention (e.g., potassium supplementation for hypokalemia) or a change in concomitant therapy

- Clinically significant in the investigator's judgment 
The investigators will review all laboratory findings. Medical and scientific judgment will be exercised in deciding whether an isolated laboratory abnormality should be classified as an adverse event. All relevant adverse event information will be communicated to a participant's primary health care provider as necessary.

\section{Data Safety Monitoring Committee}

Internal safety monitoring and review of safety events will be conducted by a Medical Research Institute non-study Researcher.

\section{Data Handling/ Statistical Methods}

\section{Study Power:}

34 Participants are sufficient to detect a paired difference in periostin of 0.5 standard deviation with $80 \%$ power. The conventional interpretation of an effect size of 0.5 of a standard deviation is of a medium effect size.

\section{Statistical analysis:}

The periostin levels will be plotted against time and a non-parametric scatter plot smoother will be used to determine if there is pattern of variation with time that might be usefully explored with parametric regression (eg linear, quadratic, cubic time terms). Paired t-test and mixed linear models will be used to compare the 12 week periostin level with measurements at the other times to formally compare the change with time. This analysis may need to be performed on the log-transformed scale. 


\section{Appendix XIV: Participant Information}

\section{Sheet for Study Six}

Participant Information Sheet

Study title: Periostin as a predictor of severe exacerbations in asthma (PER09)

Locality: Medical Research Institute Ethics 15/CEN/33

of New Zealand committee ref.:

Lead Prof. Richard Beasley Contact phone 048050147

investigator: number:

You are invited to take part in a study to determine whether or not periostin levels are predictive of severe asthma attacks in a group of patients with otherwise stable asthma. Whether or not you take part is your choice. If you do not want to take part, you do not have to give a reason, and it will not affect the care you receive. If you do want to take part now, but change your mind later, you can pull out of the study at any time. You are being asked to take part in this study, as you have previously been involved in the New Zealand Respiratory Health Survey, or the Longitudinal Study of Serum Periostin Levels in Asthma (Periostin 2), run at the Medical Research Institute of New Zealand.

You will not be eligible to take part in this study if any of the following applies to you:

- You are known to be pregnant or actively trying to conceive

- You have had surgery in the last 3 months

- You have had a bone fracture in the last 3 months

The Participant Information Sheet will help you decide if you would like to take part. It sets out why we are doing the study, what your participation would involve, what the benefits and risks to you might be, and what would happen after the study ends. We 
will go through this information with you and answer any questions you may have. We expect this will take about 20 minutes. You may also want to talk about the study with other people, such as family, whānau, friends or healthcare providers. Feel free to do this.

If you agree to take part in this study, you will be asked to sign the Consent Form on the last page of this document. You will be given a copy of both the Participant Information Sheet and the Consent Form to keep.

This document is 7 pages long, including the Consent Form. Please make sure you have read all the pages. If you require an interpreter, this can be arranged.

\section{Why are we doing the study?}

The protein periostin has been found to be present in a number of medical conditions, including asthma, early bone healing, progression of various cancers and kidney disease related to high blood pressure. It is easily measured (by a blood test) and in the future, it may be used to determine the status of a person's disease, including its severity, if it is progressing or if it is no longer present. Proteins like periostin that can allow doctors to assess a disease are known as biomarkers. This study is being conducted to determine whether or not periostin can be used as a predictive biomarker for severe asthma attacks in people with asthma, which may allow asthma to be better monitored and treated in the future. To find out, we would like to followup on our previous studies that you were involved in, by looking at your serum periostin level in relation to any severe asthma attacks you may have experienced, between the original periostin level being taken and your current visit as part of this study.

We aim to have a total of 230 participants involved in this study.

The Sponsor of this study is the Medical Research Institute of New Zealand. The study is being funded by Genentech Inc., a member of the Roche Group. The study has been approved by Central Health and Disability Ethics Committee, reference 15/CEN/33. 


\section{What would your participation involve?}

The Study is being conducted at the Medical Research Institute of New Zealand, based at Wellington Hospital. If you agree to be a part of the study, you will need to attend the Institute for a visit, which will take up to two hours.

\section{Visit Outline}

After reading this information sheet and signing the informed consent form, the study investigator will provide you with an Asthma Exacerbation Questionnaire, which you will fill out in their presence. The study investigator will then discuss your medical history and current medications so that a full picture is gained of your current health. With your permission, we will contact your GP and search your hospital records, to confirm your contact with healthcare services since you last visited the Medical Research Institute of New Zealand.

You will be asked to bring in your current medications and to withhold the following prior to breathing tests:

- Withhold caffeine for 6 hours prior to your appointment

- Withhold cigarettes for 2 hours prior to your appointment

- Withhold food for 1 hour prior to your appointment

- You will be given two asthma questionnaires (Asthma Quality of Life Questionnaire and Asthma Control Questionnaire-7) which you will fill out in the presence of the study investigator.

The following tests will then be carried out:

- We will measure nitric oxide (a gas that you normally breathe out) by asking you to breathe into a tube.

- To check the health and function of your lungs, you will be asked to do some breathing exercises. You will then follow the instructions given to you by the investigator for a few minutes. This test will be done so that three of your best measurements are recorded. This may take up to eight measurements (or more if you agree) in total, so that we can record the best results. 
- We will take a small blood sample, to measure your full blood count, serum IgE, creatinine, urea, electrolytes and your serum periostin level.

If for any reason you cannot complete the tests (e.g. due to not withholding caffeine etc) at the visit, you can go through the consent process and can attend for a second visit to complete the testing on a different day.

\section{What are the possible benefits and risk to you of participating?}

\section{Benefits}

There will be no direct benefit to you in participating in this study. You will however be part of research that may benefit yourself and others in the future.

\section{Study Procedure Risks and Side Effects}

\section{Blood samples}

You may experience some discomfort during the taking of a blood sample and there is always the risk of bleeding, swelling and bruising at the site of the needle during sampling. All samples will be taken by trained staff. Around 1-2 tablespoons of blood will be taken. A local laboratory will analyse your full blood count, creatinine, urea, electrolytes and serum IgE. Periostin will be analysed at a central laboratory outside New Zealand, by Genentech. This laboratory is:

Covance Central Laboratory Services

8211 Scicor Drive

Indianapolis

Indiana 46214

USA

Your blood samples will be coded with a unique subject number and your name will not be used to identify the samples. The samples analysed locally will be destroyed by the laboratory once the results are reported. The samples sent to Genentech may be stored for 5 years after the study has ended. You may hold beliefs about a sacred and shared value of all or any tissue samples removed. The cultural issues associated with sending your samples overseas and/or storing your tissue and/or undertaking genetic 
analysis on them should be discussed with your family/whanau as appropriate. There are a range of views held by Māori around these issues; some Iwi disagree with the storage of samples and genetic testing citing whakapapa and advise their people to consult prior to participation in research where this occurs. However, it is acknowledged that individuals have the right to choose.

\section{Breathing Exercises}

You may feel some discomfort during or after performing the breathing exercises, however this will be transient and you will be monitored constantly throughout the tests by trained technicians/study investigators. You will be seated at all times for the tests.

\section{Cost}

There will be no cost to you to take part in this study and you will be compensated for your time and travel expenses to attend your study visits ( $\$ 50$ per visit).

\section{General Health Care}

Your general health care remains with your GP throughout the time you are in the study and if you experience any problems, you should contact your usual health care provider. As part of the informed consent process, we will ask for your permission to inform your GP of any unexpected clinically significant findings we become aware of during the course of the study. As part of the study, you will be seen by study investigators, study doctors and trained staff who will conduct all the study specific assessments.

\section{What would happen if you were injured in the study?}

If you were injured as a result of treatment given as part of this study, which is unlikely, you may not be eligible for compensation from ACC. However, compensation would be available from the study's sponsor (Medical Research Institute of New Zealand). You would be able to take action through the courts if you disagreed with the amount of compensation provided. 
If you have private health or life insurance, you may wish to check with your insurer that taking part in this study will not affect your cover.

\section{What are the rights of participants in the study?}

Your participation is entirely voluntary (your choice). You do not have to take part in this study, and if you choose not to take part it will in no way affect your future health care. If you do agree to take part, you are free to withdraw from the study at any time, without having to give a reason.

\section{Confidentiality and Data Privacy}

If you decide to participate, the study doctor and relevant Institute staff will collect medical and personal information about you, including from your hospital and GP records, as part of doing the study. By agreeing to take part in this research, you will allow your medical information and results to be seen by people who check that the research was done properly, for example the ethics committee and study monitor. Nothing that could personally identify you will be used in any reports on this study, or provided as part of future studies. Your personal information (for example name, sex, age and medical conditions) and other information will be identified by a unique number (i.e. coded).

The study records will be stored securely during the course of the study and once it is completed. After all participants have completed the study and the data has been monitored, the records will be archived for a minimum of 10 years as per New Zealand regulations. The records will then be securely destroyed.

\section{What will happen after the study ends, or if you pull out?}

Once the study has finished and the data has been analysed you will be able to find out the results from the study. You may also request a copy of your individual lung function results. If you pull out of the study, you may request that we do not use any of your data as part of the results. The sponsor may stop the study at any time. You may be asked to leave the trial, for the following reasons: 
- In the Investigator's opinion it would not be in your best interest to continue in the study

- Any safety concerns

- You do not follow instructions during the study visit

Where can you go for more information about the study, or to raise concerns or complaints?

If you have any questions, concerns or complaints about the study at any stage, you can contact the study investigator:

Name: $\quad$ Ruth Semprini

Phone: $\quad 048050232$

E-mail: $\quad$ ruth.semprini@mrinz.ac.nz

For Māori health support, please contact:

Whānau Care Services, Cultural Care Centre, Level 2 Wellington Hospital

Phone: $\quad 0800999442$ or 048060948

E-mail: $\quad$ wcs@ccdhb.org.nz

If you want to talk to someone who is not involved with the study, you can contact an independent health and disability advocate on:

Phone: $\quad 0800555050$

Fax: $\quad 08002$ SUPPORT (0800 2787 7678)

E-mail: $\quad$ advocacy@hdc.org.nz

You can also contact the Health and Disability Ethics Committee (HDEC) that approved this study on:

Phone: $\quad 08004$ ETHICS (0800 4384 427)

E-mail: $\quad$ hdecs@moh.govt.nz 


\section{Consent Form}

Study Title: Periostin as a predictor for a severe exacerbation in asthma (PER09) Participant Number:

Please tick to indicate you consent to the following. If you need an interpreter, please tell us:

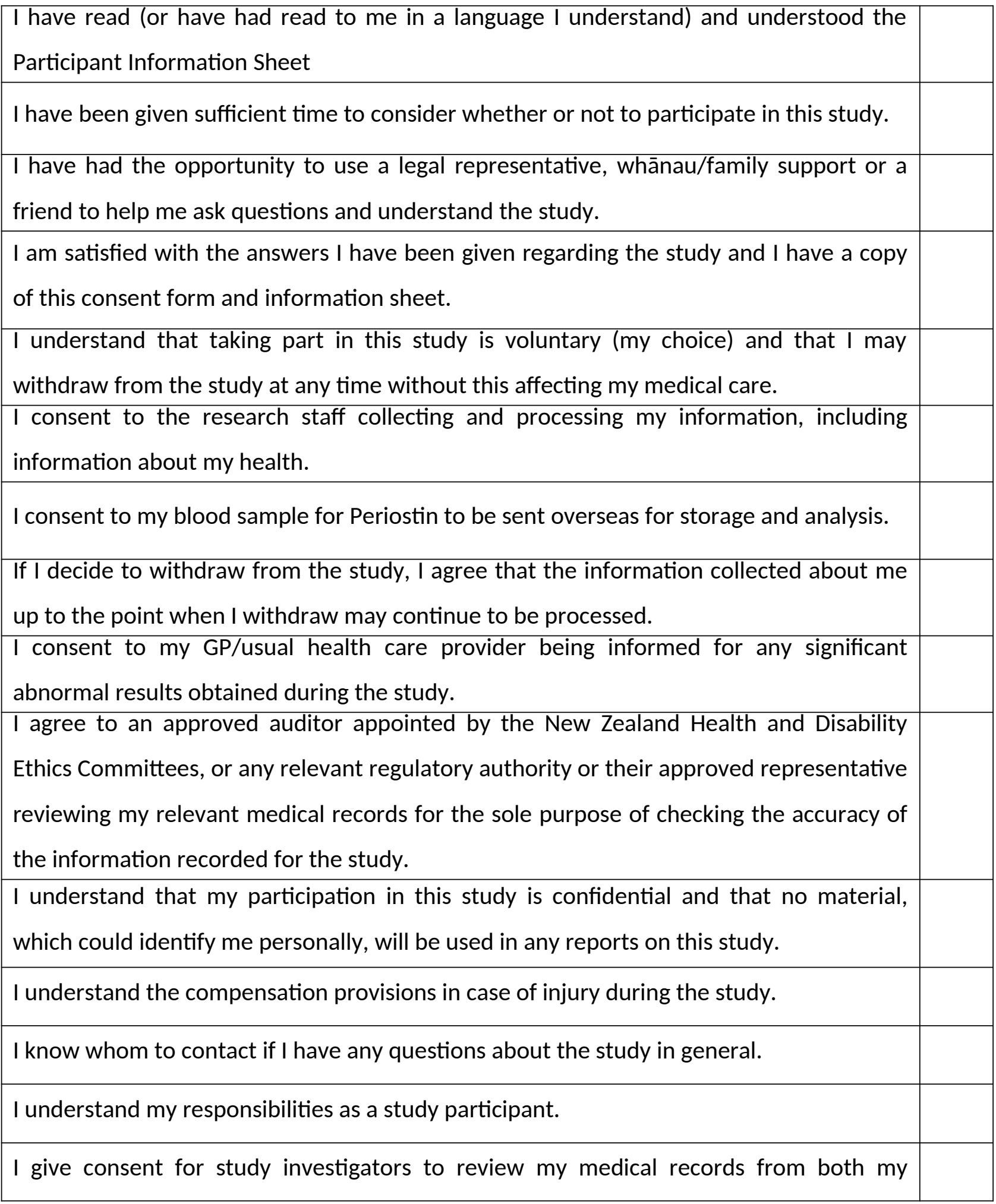


GP/usual health care provider and hospital records to corroborate my use of healthcare since my last visit to the Medical Research Institute of New Zealand.

I give consent for the study investigators to inform my GP/usual health care provider of my participation in this study.

\begin{tabular}{|l|l|l|}
\hline \multicolumn{1}{|c|}{ YES } & NO \\
\hline I wish to receive a summary of the results from the study. & & \\
\end{tabular}

Declaration by participant:

I hereby consent to take part in this study

Participant name (print)

Participant signature

Date

Declaration by member of research team:

I have given a verbal explanation of the research project to the participant, and have answered the participant's questions about it. I believe that the participant understands the study and has given informed consent to participate.

$\overline{\text { Name of person conducting informed consent discussion }}$

(print)

Signature of person conducting informed consent Date discussion 


\title{
Appendix XV: Protocol for Study Six
}

\author{
Protocol \\ Periostin as a predictor of severe exacerbations in asthma \\ Protocol No.: PER09
}

UTN: U1111-1167-8494

ANZCTRN: ACTRN12615000503527

\section{Rationale}

Asthma is a heterogenous and common condition. New Zealand has some of the highest rates of childhood asthma in the world. ${ }^{242}$ The economic burden of such a common condition has been estimated at around NZ\$800 million per annum with around 8,000 hospital admissions and 2800 day cases. ${ }^{242}$ Asthma is primarily an inflammatory condition featuring wheeze, cough and breathlessness. The main pathway implicated in asthma airway inflammation is that of the Th2 inflammatory pathway. ${ }^{51}$ Whilst eosinophilic airway inflammation is upregulated by IL-5, periostin is induced by IL-4 and IL-13 and has been shown to be upregulated in the airways of severe asthmatics. ${ }^{97}$ Traditionally corticosteroids have been used to treated airway inflammation found in asthma and are particularly effective in asthmatics where their inflammation is driven by airway eosinophilia. ${ }^{243}$ However roughly five to ten per cent of asthmatic patients remain uncontrolled despite high doses of inhaled and/or ingested corticosteroids. ${ }^{244} \mathrm{~A}$ biomarker that correlates with asthma severity would be useful both in terms of disease monitoring but also tailoring treatment to that of the individual patient.

Periostin is an extracellular matrix protein encoded by the POSTN gene (an IL-4/IL-13 inducible gene) and is thought to be an adhesion molecule as it possesses four fasciclin I domains. ${ }^{16}$ It has been shown to have functions in bone and tissue repair, oncology, cardiovascular and respiratory systems and in various other inflammatory settings. ${ }^{103}$ The secretion of periostin by lung fibroblasts, after stimulation with IL-13, results in 
subepithelial fibrosis. ${ }^{16}$ Periostin is thought to enhance fibrosis in the airways by binding to other extracellular proteins such as collagen, fibronectin and tenascin C. ${ }^{241}$ Periostin may also have a role in other TH2 driven inflammation such as nasal polyps, eosinophilic oesophagitis, allergy rhinitis, chronic sinusitis and atopic dermatitis.

Periostin has previously been shown to be useful as a biomarker of $\mathrm{TH} 2$ and eosinophilic inflammation. ${ }^{97,98}$ A highly specific serum assay has been produced which allows levels of periostin in peripheral blood to be tested. Using periostin as a systemic biomarker of airway eosinophilia would provide a minimally invasive monitoring technique which could be used to identify asthmatics who are at high risk of severe exacerbations and, as a result, justify expensive monoclonal antibody treatment which are known to reduce the risk of severe asthma exacerbations. ${ }^{245}$ This study will assess the use of periostin as a predictor of severe asthma exacerbations in pre-existing cohorts of adults with a diagnosis of asthma and compare this to currently used markers such as blood eosinophil count, serum lgE and fractional exhaled nitric oxide (FeNO). The cohorts include patients across a range of disease severity.

This study has been initiated and designed by the Medical Research Institute of New Zealand in collaboration with Genentech, Inc (a member of the Roche group). The Institute is the Sponsor of this study and undertakes Sponsor responsibilities for the purposes of ethical and any applicable regulatory requirements. Genentech are fully funding the study.

\section{Objective}

To evaluate the use of serum periostin as a predictor of severe asthma exacerbations* in adults with a diagnosis of asthma.

\section{Outcomes}

\section{Primary outcome measure}

The association between serum periostin and severe asthma exacerbations in adult asthmatics. 


\section{Secondary outcome measures:}

- The associations between serum periostin, blood eosinophil count, serum IgE, FeNO levels and the following:

- The number of severe exacerbations of asthma (as defined by ATS/ERS critera), ${ }^{209}$ i.e. the use of systemic corticosteroids for at least 3 consecutive days; or hospitalisation or ED attendances because of asthma, requiring systemic corticosteroids

- The number of hospitalisations or ED attendances because of asthma, requiring systemic corticosteroids

- The number of courses of systemic corticosteroids taken (prescribed or selfadministered)

These exacerbation outcomes are measured from the time of the baseline assessment in the NZRHS and PERO2 studies to the time of reassessment, between one and five years later.

The associations between serum periostin, blood eosinophil count, serum IgE, FeNO levels and the following:

- Changes in asthma treatment (in accordance with stepwise approach to asthma treatment in GINA guidelines) ${ }^{1}$

- Change in $\mathrm{FEV}_{1}(\mathrm{~mL} /$ year)

\section{Exploratory outcome measures}

- Other immune mediated biomarkers

- DNA-based biomarker analyses

${ }^{*}$ Severe asthma exacerbations are as defined by American Thoracic Society and the European Respiratory Society criteria 209

\section{Study Design}




\section{Participants}

356 participants with a doctor's diagnosis of asthma from pre-existing cohorts will be screened. Anticipating an attrition rate of $30 \%$, data will be collected from at least 230 participants

\section{Cohort One}

296 participants took part in the previous New Zealand Respiratory Health Survey (NZRHS) secondary phase, completing a General Health Questionnaire, spirometry assessment (FEV1 and FVC), bronchodilator reversibility, FeNO measurement and a blood test which included white cell differentials and a serum periostin level.

\section{Cohort Two}

60 participants have completed the previous longitudinal study of serum periostin levels in asthma (PERO2). At initial testing, all participants answered a General Health Questionnaire, completed spirometry assessment (FEV1 and FVC), had FeNO levels measured and a blood test for white cell differentials and serum periostin level.

\section{Inclusion Criteria}

- NZRHS phase two or PER02 participants

- Able to provide written informed consent

\section{Exclusion Criteria}

- Known Pregnancy*

- Surgery within last 3 months, determined at investigator discretion (this includes all major surgery requiring general anaesthetic, dental extractions and root canal procedures. This does not include minor procedures, including but not limited to mole or wart removal, dental fillings etc)

- Bone fracture within the last 3 months

- Active (current, or within the 3 weeks prior to the visit) upper or lower respiratory tract infection

- Any other safety concern at the investigator's discretion 
* If a potential subject is aware they are currently pregnant, or actively trying to conceive, they will be excluded from the study as pregnancy can affect FeNO, eosinophil and spirometry results. No pregnancy test will be performed on women of childbearing potential as there are no safety concerns relating to this study.

Participants may be re-screened at a suitable time should it become apparent they cannot fulfil eligibility criteria at their initial visit (e.g. if they currently have an upper respiratory tract infection).

\section{Study Procedures}

\section{Study Visits}

Participants will attend the MRINZ for one visit. During this visit, they will be asked to complete:

- Written informed consent, including consent to retrospectively review medical records through hospital and primary health care providers to corroborate the events described by the participants

- Asthma Control Questionnaire (ACQ7)

- Juniper Asthma Quality of Life Questionnaire (AQLQ)

- Body Mass Index (BMI) measurement

- Spirometry assessment (FEV1 and FVC)

- FeNO measurement

- Laboratory samples for measurement of:

- Full blood count (including white cell differentials)

- Urea, electrolytes and creatinine

- Serum IgE

- Serum periostin level

- Serum and DNA based biomarker analyses will also be conducted on samples collected for the Roche Clinical Repository (RCR). These samples will only be collected from participants who provide consent on the RCR informed consent 
form which is a separate consent to the main study (see Optional samples for Roche Clinical Repository).

Asthma and other respiratory-related events over the intervening time period, since their last attendance at the MRINZ will be documented. Such events will include, but not be limited to:

- Medication changes

- Unplanned primary health care appointments

- Emergency Department visits

- Hospitalisations for asthma

- The number of courses of prednisone completed by the participant

All participants will have their hospital medical records and their primary health care records reviewed by a member of the study team, with their informed consent, to corroborate the information gathered. Documented contact with the participant will over-ride participant recall when appropriate. Participant's recall regarding their contact with medical professionals during an asthma exacerbation will be particularly important where this has happened outside of Wellington, New Zealand and cannot be corroborated with local records.

\section{Informed Consent}

Informed consent will be obtained according to ethical and GCP guidelines, and will take place at the MRINZ. Each participant will sign an informed consent form upon visiting MRINZ, prior to conducting the procedures listed above.

\section{Respiratory Health Questionnaires}

The Asthma Quality of Life Questionnaire with Standardised Activities (AQLQ(S)) is a validated 32 question self-administered questionnaire that will be used to obtain severity of asthma in the two weeks prior to attending MRINZ. 
The Asthma Control Questionnaire (ACQ7) is a validated 7 question self-administered questionnaire that will be used to obtain severity of asthma in the 1 week prior to visiting MRINZ.

Asthma Exacerbation Questionnaire is a detailed questionnaire prompting participants to recall moderate and severe exacerbations they may have had for each twelve month period before review.

\section{Lung Function Tests}

\section{Nitric Oxide}

FeNO will be measured using a nitric oxide monitor (NIOX MINO; Aerocrine AB, Solna, Sweden) according to the ATS guidelines published in $2005^{13}$. Seated subjects will exhale fully and then inhale ambient air through a nitric oxide scrubber to total lung capacity. Subjects will then exhale against an automatically adjusting resistance to achieve a constant exhalation flow rate of $50 \mathrm{ml} / \mathrm{s}+/-\mathrm{mls}$. Resistance will be adjusted so that an upper airway pressure of $10-20 \mathrm{~cm} \mathrm{H} 2 \mathrm{O}$ is maintained throughout exhalation, sufficient to close the velum and exclude nasal air. FeNO measurements will be taken from a stable plateau in exhaled nitric oxide concentration during an exhalation. Exhalations where flow rate and plateau criteria are not met will be deemed not acceptable for measurement. One acceptable exhalation will be measured and recorded at each time point. Measurements will be made before other pulmonary function testing. Participants will avoid eating for 1 hour, smoking tobacco for 2 hours, and caffeine ingestion for 6 hours. Subjects will not be tested within 3 weeks of an upper or lower respiratory tract infection.

\section{Spirometry}

Participants will undergo pulmonary testing using a spirometer which meets the guidelines outlined by the American Thoracic Society $2005 .{ }^{184}$

All tests will be performed:

Sitting 
With nose clips on

The tests will be clearly explained to the subject before they are started and positive encouragement given at all stages of the test.

\section{Acceptability criteria}

Criteria for technically satisfactory tests:

- Coughing during the first second of the exhalation

- Glottis closure that influences the measurement

- Effort that is not maximal throughout

- Obstructed mouthpiece e.g. tongue in front of the mouthpiece

- A leak in the system or around the mouthpiece

- Excessive hesitation at the start of expiration (extrapolated volume $<5 \%$ or $150 \mathrm{ml}$ of FVC -whichever is greater.

- Early termination of test by subject

Repeatability criteria

Less than $150 \mathrm{ml}$ variation in FEV1 between attempts

Less than $150 \mathrm{ml}$ variation in FVC between attempts

Testing should be continued until 3 acceptable manoeuvres have been completed or the subject has performed 8 manoeuvres-more can be done if the subject agrees. According to ATS guidelines subjects who are unable to produce reproducible flow volume loops (reproducible flow loops are those with <150ml in variation in FEV1 and FVC) will not be excluded from analysis. In subjects who were not able to produce 3 acceptable flow volume loops comments regarding the technical acceptability of their testing will be made.

\section{Laboratory Samples}

Samples for the following tests will be analysed by a local laboratory:

- Serum IgE 
- Full blood count (including differential white cell count)

- Urea, electrolytes and creatinine

Samples will be analysed as per the laboratory's standard operating procedures.

Samples for the following tests will be sent to Genentech (or their designee) to be analysed:

- Serum periostin level

Optional samples for exploratory research (as per the RCR analysis below) will also be sent to Genentech (or their designee)

- Other immune mediated serum biomarkers

- DNA- based biomarker analyses

\section{Optional Samples for Roche Clinical Repository (RCR)}

\section{Overview of the Roche Clinical Repository}

The Roche Clinical Repository (RCR) is a centrally administered group of facilities for the long-term storage of human biologic specimens, including body fluids, solid tissues, and derivatives thereof (e.g., DNA, RNA, proteins, peptides). The collection and analysis of RCR specimens will facilitate the rational design of new pharmaceutical agents and the development of diagnostic tests, which may allow for individualized drug therapy for patients in the future. Specimens for the RCR will be collected from patients who give specific consent to participate in this optional research.

\section{Approval by the Institutional Review Board or Ethics Committee}

Sampling for the RCR is contingent upon the review and approval of the exploratory research and the RCR specific Informed Consent Form (ICF) by the New Zealand Health and Disability Ethics Committees.

\section{Optional RCR Sample Collection}

For sampling procedures, storage conditions, and shipment instructions, see the laboratory manual. RCR specimens will be destroyed no later than 15 years after the 
date of final closure of the associated clinical database. The RCR storage period will be in accordance with the IRB/EC-approved ICF and applicable laws (e.g. health authority requirements). The dynamic biomarker specimens will be subject to confidentiality standards, i.e. by coding each participant enrolled in the study through assignment of a unique subject identification number. This means that participant names are not included in data sets that are transmitted to any location outside of the MRINZ.

\section{Confidentiality}

Data generated from RCR specimens must be available for inspection upon request by representatives of national and local health authorities, and Roche monitors, representatives, and collaborators, as appropriate. Participant medical information associated with RCR specimens is confidential and may only be disclosed to third parties as permitted by the ICF (or separate authorization for use and disclosure of personal health information) signed by the participant, unless permitted or required by law. Data derived from RCR specimen analysis on individual participants will generally not be provided to study investigators unless a request for research use is granted. The aggregate results of any research conducted using RCR specimens will be available in accordance with the effective Roche policy on study data publication. Any inventions and resulting patents, improvements, and/or know-how originating from the use of the RCR data will become and remain the exclusive and unburdened property of Roche, except where agreed otherwise.

\section{Consent to Participate in the Roche Clinical Repository}

There will be a separate ICF which will contain information that addresses participation in the RCR. The investigator or authorized designee will explain to each participant the objectives, methods, and potential hazards of participation in the RCR. Participants will be told that they are free to refuse to participate and may withdraw their specimens at any time and for any reason during the storage period. A separate, specific signature will be required to document a participant's agreement to provide optional RCR specimens. Participants who decline to participate will not provide a separate signature. In the event of an RCR participant's death or loss of competence, the participant's specimens and data will continue to be used as part of the RCR research. 


\section{Withdrawal from the Roche Clinical Repository}

Participants who give consent to provide RCR specimens have the right to withdraw their specimens from the RCR at any time for any reason. If a participant wishes to withdraw consent to the testing of his or her specimens, the investigator will inform Genentech in writing of the participant's wishes. The participant will be provided with instructions on how to withdraw consent after the trial is closed. A participant's withdrawal from this study does not, by itself, constitute withdrawal of specimens from the RCR. Likewise, a participant's withdrawal from the RCR does not constitute withdrawal from this study.

\section{Monitoring and Oversight}

RCR specimens will be tracked in a manner consistent with Good Clinical Practice (GCP) by a quality-controlled, auditable, and appropriately validated laboratory information management system, to ensure compliance with data confidentiality as well as adherence to authorized use of specimens as specified in this protocol and in the ICF. Roche and MRINZ monitors and auditors will have direct access to appropriate parts of records relating to patient participation in the RCR for the purposes of verifying the data provided to Roche, as necessary. The site will permit monitoring, audits, IRB/EC review, and health authority inspections by providing direct access to source data and documents related to the RCR samples.

\section{Safety Monitoring}

\section{Adverse Event Reporting}

For the purposes of this study, the study duration is considered to start once the informed consent form has been signed by the participant. The study period will be deemed to have ended when the study participant completes their visit and leaves MRINZ premises. The MRINZ will be responsible for reviewing the blood tests taken during the study visit and reporting any clinically significant abnormalities to the participant's GP/ primary healthcare provider, as necessary. 


\section{Adverse Events}

An adverse event is any untoward medical occurrence in a study subject temporally associated with the testing as part of the study. An adverse event can therefore be any unfavourable and unintended sign, symptom or disease temporally associated with the testing carried out in the study. Adverse event data will be collected by investigators during the study visits and after reviewing the test results.

\section{Serious Adverse Events (SAEs)}

For the purposes of this study the following events will be considered to be SAEs:

- Death

- Life-threatening event

- Permanently disabling or incapacitating event

- Hospitalisation. Hospitalisation for the purposes of SAE reporting is defined as an admission to hospital and does not include a presentation to the Emergency Department followed by discharge without admission or an admission for elective reasons

- Any other significant medical event considered serious by the study investigator

All SAEs will be reported to the NZ HDEC and regulatory authorities as per current guidelines. SAEs and AEs will be followed up until resolution, or judged to be permanent. All SAEs will be recorded by the MRINZ (as study sponsor) within 24 hours of the study investigators becoming aware of the event.

\section{Abnormal Laboratory Values}

Not every laboratory abnormality qualifies as an adverse event. A laboratory test result should be reported as an adverse event if it meets any of the following criteria:

- Accompanied by clinical symptoms

- Results in a medical intervention (e.g., potassium supplementation for hypokalemia) or a change in concomitant therapy

- Clinically significant in the investigator's judgment 
The investigators will review all laboratory findings. Medical and scientific judgment will be exercised in deciding whether an isolated laboratory abnormality should be classified as an adverse event. All relevant adverse event information will be communicated to a participant's primary health care provider as necessary.

\section{Data Safety Monitoring Committee}

Internal safety monitoring and review of safety events will be conducted by a Medical Research Institute non-study Researcher.

\section{Data Handling/Statistical Methods}

\section{Study Power}

356 participants will be approached to take part in the study, based on the number of participants recruited from the NZRHS and PER02 studies. Assuming a 30\% attrition rate, it is envisaged that 230 participants will be enrolled into this study.

\section{Statistical analyses:}

This study will estimate the associations between baseline serum periostin and:

- The number of severe asthma exacerbations as defined by ATS/ERS criteria, i.e. the use of systemic corticosteroids, or an increase from a stable maintenance dose, for at least 3 days; or hospitalisation or ED visit because of asthma requiring systemic corticosteroids. This will be treated as a count variable for analysis.

- The number of hospitalisations or ED visits because of asthma, requiring systemic corticosteroids.

- The number of courses of systemic corticosteroids taken (either prescribed or self-administered). This will be treated as a count variable for analysis.

- FEV1, adjusted for baseline. This will be treated as a continuous variable for analysis.

- Treatment requirements, categorised by GINA treatment steps. This will be treated as an ordinal variable for analysis. 
- Detailed data description using summary statistics, contingency tables, and plots will be used for both the explanatory and response variables including, where appropriate descriptions of change with time. Our experience with analysis of Periostin, FeNO, and serum IgE, are they are typically highly skew and better analysed on the logarithm transformed scale.

- For illustrative purposes data descriptions based on tertiles will also be calculated.

- The statistical analysis will be appropriate to the response variable data type.

- For the response variables treated as counts (exacerbations, hospital or ED admissions, and courses of steroids) this will be by Poisson regression with an offset for the time observation. It is possible that most of the participants will have no or only one count of a response variable and in this case it may be simpler to analyse the associations using logistic regression, using levels of none versus any.

- For the response variable treated as continuous, FEV1, regression will be used, including the baseline FEV1 as a covariate.

- For the response variable treated as ordinal, GINA asthma step, proportional odds (ordinal) regression will be used. If in the event there are mainly two asthma step levels achieved it may be simpler to analyse associations using logistic regression with GINA asthma step as a two level response variable.

- Periostin will be used as an explanatory variable in both univariate and multivariate analyses.

- In the univariate analyses, Periostin or logarithm Periostin, will be used as the sole explanatory variable

- The other Th2-related variables: eosinophil count, IgE, and FeNO, will also be used in univariate analyses with each as a sole explanatory variable

- Finally multivariate models will be estimated with all of eosinophil count, IgE, and FeNO, to explore if Periostin adds important explanatory power to prediction of poor asthma outcomes.

This is an exploratory analysis with a large number of statistical tests and although $95 \%$ confidence intervals, corresponding to a two-sided type I error rate of $5 \%$, will be used, results will be interpreted cautiously because of multiple statistical testing and 
uncertainty as to what, for some variables, might constitute a clinically meaningful association. 
Appendix XVI: Asthma Exacerbation

\author{
Questionnaire
}




\section{Asthma Exacerbation Questionnaire}

MEDICAL RESEARCH

OF NEW ZEALAND

Name:

PER09

participant

ID:

Genentech

ID:

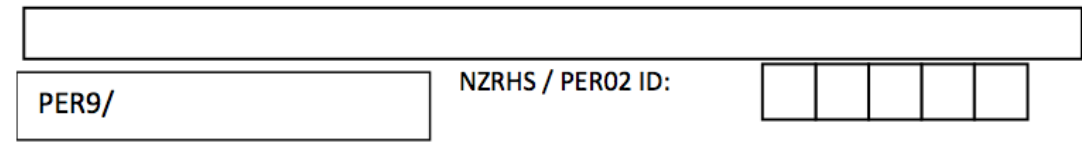

Today's date:
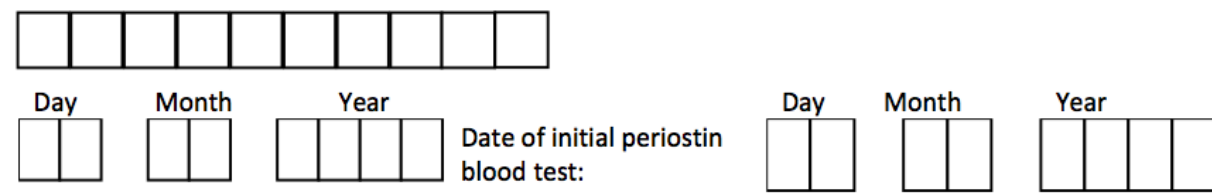

\section{A. General Questions}

1. Date of Birth

Day
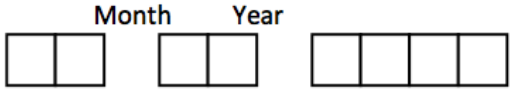

2. Are you male or female?

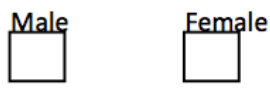

3. Your regular GP Name and Practice Name:

4. Have you been diagnosed with any other respiratory conditions other than asthma (e.g COPD, bronchiectasis etc)? If so, please state:

B. Asthma Health

5. What is your current treatment for your asthma? Note to investigator - medication that the participant is prescribed AND is currently taking.

\begin{tabular}{|l|l|l|l|l|l|}
\hline Medication name & Dose & Units & Route & Frequency & Date started \\
\hline & & & & & \\
\hline & & & & & \\
\hline & & & & & \\
\hline & & & & & \\
\hline & & & & & \\
\hline & & & & & \\
\hline
\end{tabular}

PER09 Asthma Exacerbation Questionnaire

Dated: 16th June 2015

Version 2.2 


\section{Hospital attendances in $\mathbf{2 0 1 0}$}

6. In 2010, how many times did you visit hospital (including ED visits) for your asthma? Please list all hospitals below. NB 'steroids' may have been given as tablets, injection or via a drip. No attendances:

\begin{tabular}{|c|c|c|c|c|c|c|c|}
\hline Date & Hospital Name & $\begin{array}{c}\text { Admitted? } \\
(\mathrm{Y} / \mathrm{N})\end{array}$ & $\begin{array}{c}\text { Attendance } \\
<6 \mathrm{~h} ?(\mathrm{Y} / \mathrm{N})\end{array}$ & $\begin{array}{c}\text { Attendance } \\
6-24 \mathrm{~h} ? \\
(\mathrm{Y} / \mathrm{N})\end{array}$ & $\begin{array}{c}\text { Attendance } \\
>24 \mathrm{~h} ? \\
(\mathrm{Y} / \mathrm{N})\end{array}$ & $\begin{array}{c}\text { Steroids? } \\
(\mathrm{Y} / \mathrm{N})\end{array}$ & $\begin{array}{c}\text { Admitted to } \\
\text { ICU? (Y/N) }\end{array}$ \\
\hline & & & & & & & \\
\hline & & & & & & & \\
\hline & & & & & & & \\
\hline
\end{tabular}

\section{General Practitioner (GP) Attendances in $\mathbf{2 0 1 0}$}

7. Did you see your GP in $\mathbf{2 0 1 0}$ due to worsening of your asthma symptoms as an unscheduled visit? If NO, please go straight to Section < E >.

If YES, please provide details:

\begin{tabular}{|l|l|l|l|}
\hline Date & GP Practice Name & $\begin{array}{l}\text { Steroids given for 3 } \\
\text { days or longer? }(\mathrm{Y} / \mathrm{N})\end{array}$ & $\begin{array}{l}\text { Hospital attendance as } \\
\text { a result of GP visit? } \\
(\mathrm{Y} / \mathrm{N})\end{array}$ \\
\hline & & & \\
\hline & & & \\
\hline & & & \\
\hline & & & \\
\hline & & & \\
\hline
\end{tabular}

\section{E. Self-management in $\mathbf{2 0 1 0}$}

8. Did you have an asthma attack in $\mathbf{2 0 1 0}$ where you took at least $\mathbf{3}$ days of oral steroids, but did not obtain medical review? If NO, please go to Section <F $>$. If YES, please provide details:

\begin{tabular}{|l|l|l|}
\hline Date & Treatment taken (please tick) \\
\hline & None & \\
\cline { 2 - 2 } & At least 3 days of steroids & \\
\hline & None & \\
\cline { 2 - 2 } & At least 3 days of steroids & \\
\hline & None & \\
\cline { 2 - 3 } & At least 3 days of steroids & \\
\hline & None & \\
\cline { 2 - 3 } & At least 3 days of steroids & \\
\hline & None & \\
\cline { 2 - 3 } & At least 3 days of steroids & \\
\hline
\end{tabular}




\section{F. Hospital attendances in $\mathbf{2 0 1 1}$}

9. In 2011, how many times did you visit hospital (including ED visits) for your asthma? Please list all hospitals below. NB 'steroids' may have been given as tablets, injection or via a drip. No attendances:

\begin{tabular}{|c|c|c|c|c|c|c|c|}
\hline Date & Hospital Name & $\begin{array}{c}\text { Admitted? } \\
(\mathrm{Y} / \mathrm{N})\end{array}$ & $\begin{array}{c}\text { Attendance } \\
<6 \mathrm{~h} ?(\mathrm{Y} / \mathrm{N})\end{array}$ & $\begin{array}{c}\text { Attendance } \\
6-24 \mathrm{~h} ? \\
(\mathrm{Y} / \mathrm{N})\end{array}$ & $\begin{array}{c}\text { Attendance } \\
>24 \mathrm{~h} ? \\
(\mathrm{Y} / \mathrm{N})\end{array}$ & $\begin{array}{c}\text { Steroids? } \\
(\mathrm{Y} / \mathrm{N})\end{array}$ & $\begin{array}{c}\text { Admitted to } \\
\text { ICU? (Y/N) }\end{array}$ \\
\hline & & & & & & & \\
\hline & & & & & & & \\
\hline
\end{tabular}

\section{G. General Practioner (GP) Attendances in 2011}

10. Did you see your GP in 2011 due to worsening of your asthma symptoms as an unscheduled visit? If NO, please go straight to Section $<\mathrm{H}>$.

If YES, please provide details:

\begin{tabular}{|l|l|l|l|}
\hline Date & GP Practice Name & $\begin{array}{l}\text { Steroids given for 3 } \\
\text { days or longer? }(\mathrm{Y} / \mathrm{N})\end{array}$ & $\begin{array}{l}\text { Hospital attendance as } \\
\text { a result of GP visit? } \\
(\mathrm{Y} / \mathrm{N})\end{array}$ \\
\hline & & & \\
\hline & & & \\
\hline & & & \\
\hline & & & \\
\hline & & & \\
\hline
\end{tabular}

H. Self-management in $\mathbf{2 0 1 1}$

11. Did you have an asthma attack in 2011 where you took at least 3 days of oral steroids, but did not obtain medical review? If NO, please go to Section $<\mathrm{l}\rangle$.

If YES, please provide details:

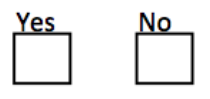

\begin{tabular}{|l|l|l|}
\hline Date & Treatment taken (please tick) \\
\hline \multirow{4}{*}{} & None & \\
\cline { 2 - 2 } & At least 3 days of steroids & \\
\hline & None & \\
\cline { 2 - 2 } & At least 3 days of steroids & \\
\hline & None & \\
\cline { 2 - 2 } & At least 3 days of steroids & \\
\hline & None & \\
\cline { 2 - 3 } & At least 3 days of steroids & \\
\hline & None \\
\hline & At least 3 days of steroids & \\
\hline
\end{tabular}


I. Hospital attendances in $\mathbf{2 0 1 2}$

12. In 2012, how many times did you visit hospital (including ED visits) for your asthma? Please list all hospitals below. NB 'steroids' may have been given as tablets, injection or via a drip.

No attendances:

\begin{tabular}{|c|c|c|c|c|c|c|c|}
\hline Date & Hospital Name & $\begin{array}{c}\text { Admitted? } \\
(\mathrm{Y} / \mathrm{N})\end{array}$ & $\begin{array}{c}\text { Attendance } \\
<6 \mathrm{~h} ?(\mathrm{Y} / \mathrm{N})\end{array}$ & $\begin{array}{c}\text { Attendance } \\
6-24 \mathrm{~h} ? \\
(\mathrm{Y} / \mathrm{N})\end{array}$ & $\begin{array}{c}\text { Attendance } \\
>24 \mathrm{~h} ? \\
(\mathrm{Y} / \mathrm{N})\end{array}$ & $\begin{array}{c}\text { Steroids? } \\
(\mathrm{Y} / \mathrm{N})\end{array}$ & $\begin{array}{c}\text { Admitted to } \\
\text { ICU? (Y/N) }\end{array}$ \\
\hline & & & & & & & \\
\hline & & & & & & & \\
\hline
\end{tabular}

\section{J. General Practioner (GP) Attendances in 2012}

13. Did you see your GP in $\mathbf{2 0 1 2}$ due to worsening of your asthma symptoms as an unscheduled visit? Yes If NO, please go straight to Section < K >.

If YES, please provide details:

\begin{tabular}{|l|l|l|l|}
\hline Date & GP Practice Name & $\begin{array}{l}\text { Steroids given for 3 } \\
\text { days or longer? }(\mathrm{Y} / \mathrm{N})\end{array}$ & $\begin{array}{l}\text { Hospital attendance as } \\
\text { a result of GP visit? } \\
(\mathrm{Y} / \mathrm{N})\end{array}$ \\
\hline & & & \\
\hline & & & \\
\hline & & & \\
\hline & & & \\
\hline & & & \\
\hline
\end{tabular}

\section{K. Self-management in $\mathbf{2 0 1 2}$}

14. Did you have an asthma attack in 2012 where you took at least 3 days of oral steroids, but did not obtain medical review? If NO, please go to Section $\langle\mathrm{L}\rangle$.

If YES, please provide details:

\begin{tabular}{|l|l|l|}
\hline Date & Treatment taken (please tick) \\
\hline & None & \\
\cline { 2 - 2 } & At least 3 days of steroids & \\
\hline & None & \\
\cline { 2 - 2 } & At least 3 days of steroids & \\
\hline & None & \\
\cline { 2 - 2 } & At least 3 days of steroids & \\
\hline & None & \\
\cline { 2 - 3 } & At least 3 days of steroids & \\
\hline & None \\
\hline & At least 3 days of steroids & \\
\hline
\end{tabular}




\section{Hospital attendances in $\mathbf{2 0 1 3}$}

15. In 2013, how many times did you visit hospital (including ED visits) for your asthma? Please list all hospitals below. NB 'steroids' may have been given as tablets, injection or via a drip.

No attendances:

\begin{tabular}{|c|c|c|c|c|c|c|c|}
\hline Date & Hospital Name & $\begin{array}{c}\text { Admitted? } \\
(\mathrm{Y} / \mathrm{N})\end{array}$ & $\begin{array}{c}\text { Attendance } \\
<6 \mathrm{~h} ?(\mathrm{Y} / \mathrm{N})\end{array}$ & $\begin{array}{c}\text { Attendance } \\
6-24 \mathrm{~h} ? \\
(\mathrm{Y} / \mathrm{N})\end{array}$ & $\begin{array}{c}\text { Attendance } \\
>24 \mathrm{~h} ? \\
(\mathrm{Y} / \mathrm{N})\end{array}$ & $\begin{array}{c}\text { Steroids? } \\
(\mathrm{Y} / \mathrm{N})\end{array}$ & $\begin{array}{c}\text { Admitted to } \\
\text { ICU? (Y/N) }\end{array}$ \\
\hline & & & & & & & \\
\hline & & & & & & & \\
\hline & & & & & & & \\
\hline
\end{tabular}

\section{General Practioner (GP) Attendances in 2013}

16. Did you see your GP in 2013 due to worsening of your asthma symptoms as an unscheduled visit? If NO, please go straight to Section $\langle M\rangle$.

If YES, please provide details:

\begin{tabular}{|l|l|l|l|}
\hline Date & GP Practice Name & $\begin{array}{l}\text { Steroids given for 3 } \\
\text { days or longer? }(\mathrm{Y} / \mathrm{N})\end{array}$ & $\begin{array}{l}\text { Hospital attendance as } \\
\text { a result of GP visit? } \\
(\mathrm{Y} / \mathrm{N})\end{array}$ \\
\hline & & & \\
\hline & & & \\
\hline & & & \\
\hline & & & \\
\hline & & & \\
\hline
\end{tabular}

\section{N. Self-management in 2013}

17. Did you have an asthma attack in 2013 where you took at least 3 days of oral steroids, but did not obtain medical review? If NO, please go to Section $\langle\mathrm{N}\rangle$.

If YES, please provide details:

\begin{tabular}{|l|l|l|}
\hline Date & Treatment taken (please tick) \\
\hline & None & \\
\cline { 2 - 2 } & At least 3 days of steroids & \\
\hline & None & \\
\cline { 2 - 2 } & At least 3 days of steroids & \\
\hline & None & \\
\cline { 2 - 3 } & At least 3 days of steroids & \\
\hline & None & \\
\cline { 2 - 3 } & At least 3 days of steroids & \\
\hline & None & \\
\hline & At least 3 days of steroids & \\
\hline
\end{tabular}


O. Hospital attendances in 2014

18. In 2014, how many times did you visit hospital (including ED visits) for your asthma? Please list all hospitals below. NB 'steroids' may have been given as tablets, injection or via a drip.

No attendances:

\begin{tabular}{|c|c|c|c|c|c|c|c|}
\hline Date & Hospital Name & $\begin{array}{c}\text { Admitted? } \\
(\mathrm{Y} / \mathrm{N})\end{array}$ & $\begin{array}{c}\text { Attendance } \\
<6 \mathrm{~h} ?(\mathrm{Y} / \mathrm{N})\end{array}$ & $\begin{array}{c}\text { Attendance } \\
6-24 \mathrm{~h} ? \\
(\mathrm{Y} / \mathrm{N})\end{array}$ & $\begin{array}{c}\text { Attendance } \\
>24 \mathrm{~h} ? \\
(\mathrm{Y} / \mathrm{N})\end{array}$ & $\begin{array}{c}\text { Steroids? } \\
(\mathrm{Y} / \mathrm{N})\end{array}$ & $\begin{array}{c}\text { Admitted to } \\
\text { ICU? }(\mathrm{Y} / \mathrm{N})\end{array}$ \\
\hline & & & & & & & \\
\hline & & & & & & & \\
\hline & & & & & & & \\
\hline
\end{tabular}

P. General Practioner (GP) Attendances in 2014

19. Did you see your GP in 2014 due to worsening of your asthma symptoms as an unscheduled visit? If NO, please go straight to Section $\langle Q\rangle$

If YES, please provide details:

\begin{tabular}{|l|l|l|l|}
\hline Date & GP Practice Name & $\begin{array}{l}\text { Steroids given for } 3 \\
\text { days or longer? }(\mathrm{Y} / \mathrm{N})\end{array}$ & $\begin{array}{l}\text { Hospital attendance as } \\
\text { a result of GP visit? } \\
(\mathrm{Y} / \mathrm{N})\end{array}$ \\
\hline & & & \\
\hline & & & \\
\hline & & & \\
\hline & & & \\
\hline & & & \\
\hline
\end{tabular}

\section{Q. Self-management in 2014}

20. Did you have an asthma attack in 2014 where you took at least 3 days of oral steroids, but did not obtain medical review? If NO, please go to Section $\langle R\rangle$. If YES, please provide details:

\begin{tabular}{|l|l|l|}
\hline Date & Treatment taken (please tick) \\
\hline & None & \\
\cline { 2 - 3 } & At least 3 days of steroids & \\
\hline & None & \\
\cline { 2 - 3 } & At least 3 days of steroids & \\
\hline & None & \\
\cline { 2 - 3 } & At least 3 days of steroids & \\
\hline & None & \\
\cline { 2 - 3 } & At least 3 days of steroids & \\
\hline & None & \\
\cline { 2 - 3 } & At least 3 days of steroids & \\
\hline
\end{tabular}


R. Hospital attendances in $\mathbf{2 0 1 5}$

21. In 2015 , how many times did you visit hospital (including ED visits) for your asthma? Please list all hospitals below. NB 'steroids' may have been given as tablets, injection or via a drip. No attendances:

\begin{tabular}{|c|c|c|c|c|c|c|c|}
\hline Date & Hospital Name & $\begin{array}{c}\text { Admitted? } \\
(\mathrm{Y} / \mathrm{N})\end{array}$ & $\begin{array}{c}\text { Attendance } \\
<6 \mathrm{~h} ?(\mathrm{Y} / \mathrm{N})\end{array}$ & $\begin{array}{c}\text { Attendance } \\
6-24 \mathrm{~h} ? \\
(\mathrm{Y} / \mathrm{N})\end{array}$ & $\begin{array}{c}\text { Attendance } \\
>24 \mathrm{~h} ? \\
(\mathrm{Y} / \mathrm{N})\end{array}$ & $\begin{array}{c}\text { Steroids? } \\
(\mathrm{Y} / \mathrm{N})\end{array}$ & $\begin{array}{c}\text { Admitted to } \\
\text { ICU? (Y/N) }\end{array}$ \\
\hline & & & & & & & \\
\hline & & & & & & & \\
\hline
\end{tabular}

\section{S. General Practioner (GP) Attendances in 2015}

22. Did you see your GP in 2015 due to worsening of your asthma symptoms as an unscheduled visit? If NO, please go straight to Section $<T>$. If YES, please provide details:

\begin{tabular}{|l|l|l|l|}
\hline Date & GP Practice Name & $\begin{array}{l}\text { Steroids given for 3 } \\
\text { days or longer? }(\mathrm{Y} / \mathrm{N})\end{array}$ & $\begin{array}{l}\text { Hospital attendance as } \\
\text { a result of GP visit? } \\
(\mathrm{Y} / \mathrm{N})\end{array}$ \\
\hline & & & \\
\hline & & & \\
\hline & & & \\
\hline & & & \\
\hline & & & \\
\hline
\end{tabular}

T. Self-management in $\mathbf{2 0 1 5}$

23. Did you have an asthma attack in 2015 where you took at least 3 days of oral steroids, but did not obtain medical review? If NO, please go to Declaration. If YES, please provide details:

\begin{tabular}{|l|l|l|}
\hline Date & Treatment taken (please tick) \\
\hline & None & \\
\cline { 2 - 2 } & At least 3 days of steroids & \\
\hline & None & \\
\cline { 2 - 2 } & At least 3 days of steroids & \\
\hline & None & \\
\cline { 2 - 3 } & At least 3 days of steroids & \\
\hline & None & \\
\cline { 2 - 3 } & At least 3 days of steroids & \\
\hline & None & \\
\cline { 2 - 3 } & At least 3 days of steroids & \\
\hline
\end{tabular}

Number of severe exacerbations as reported by participant:

\section{Declaration}

Form completed by

Participant signature

Date:.

Investigator Name

Investigator Signature

Date:

PER09 Asthma Exacerbation Questionnaire

Dated: 16th June 2015

Version 2.2 


\title{
Appendix XVII: Permissions from
}

\author{
publications
}

From: "Ruth Semprini [CCDHB]" <Ruth.Semprini@ccdhb.org.nz>

Subject: Fwd: 00913703 RE: Copyright

Date: 17 August 2018 at 5:35:51 PM NZST

To: Ruth Pais <ruth.a.pais@googlemail.com>

Ruth Semprini

On 15 August 2018 at 14:52:27, Neil Castil (neil.castil@springernature.com) wrote:

Dear Ruth,

Thank you for contacting Springer Nature.

The open access articles published in BioMed

Central's journals are made available under the Creative Commons Attribution (CC-BY) license, which means they are accessible online without any restrictions and can be re-used in any way, subject only to proper attribution (which, in an academic context, usually means citation).

The re-use rights enshrined in our license agreement (http://www.biomedcentral.com/about/policies/ license-agreement) include the right for anyone to produce printed copies themselves, without formal permission or payment of permission fees. As a courtesy, however, anyone wishing to reproduce large quantities of an open access article (250+) should inform the copyright holder and we suggest a contribution in support of open access publication (see suggested contributions at http:// www.biomedcentral.com/about/policies/reprints-and- 
From: no-reply@

Subject: Thank you for your order with RightsLink / BMJ Publishing Group Ltd.

Date: 15 August 2018 at 2:30 PM

To: ruth.a.pais@gmail.com

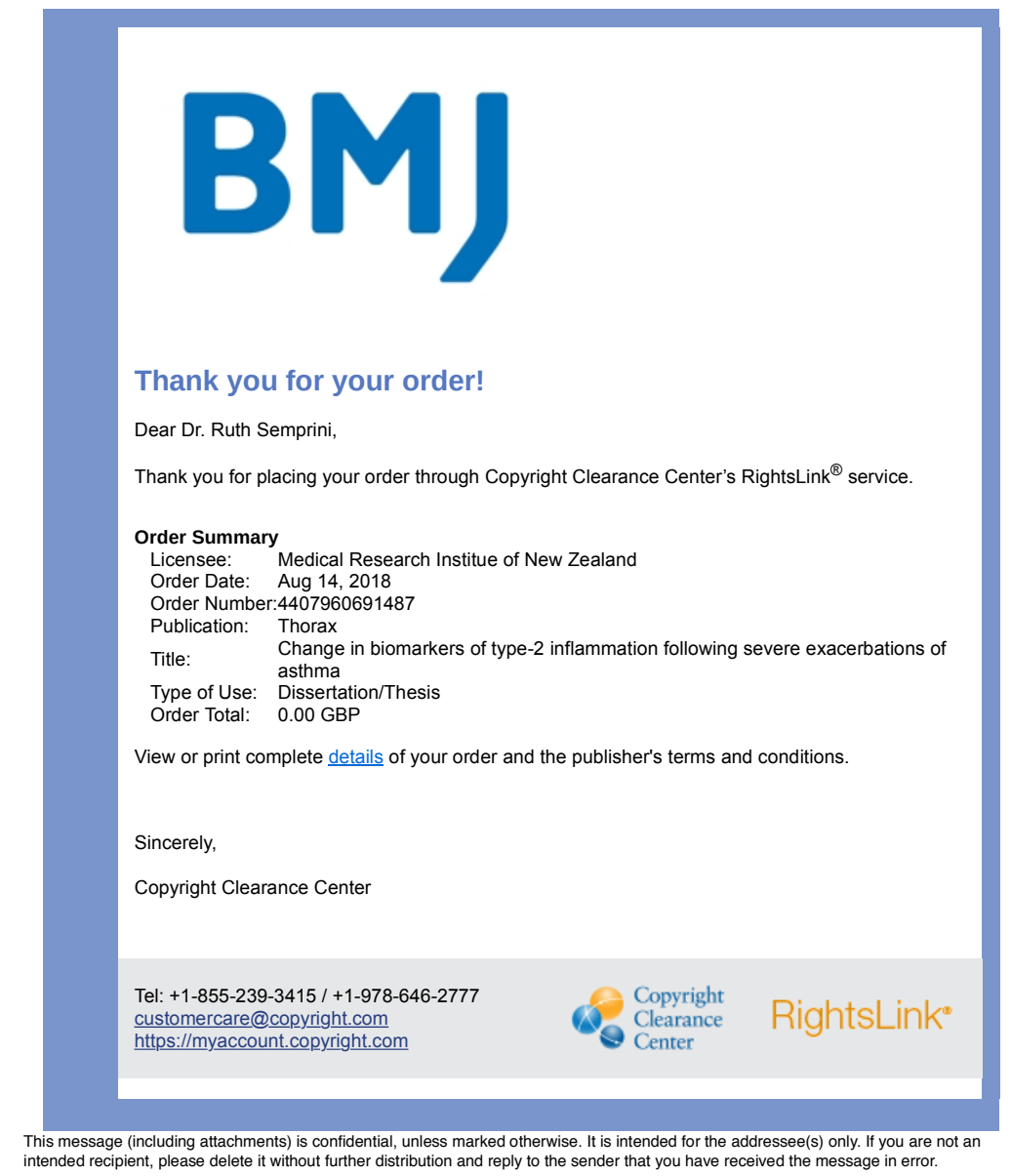




\section{Order Details}

The journal of allergy and clinical immunology

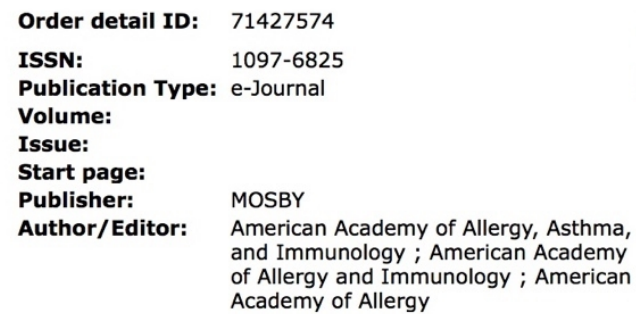

Note: This item was invoiced separately through our RightsLink service. More info

Order detail ID: 71427575

ISSN: 2213-2198

Publication Type: Journal

Volume:

Issue:

Start page:

Publisher:

Author/Editor:
Elsevier

American Academy of Allergy, Asthma, and Immunology,

\section{Permission Status: $\checkmark$ Granted}

Permission type: Republish or display content Type of use: Thesis/Dissertation

Order License Id: 4407960064652 


\section{References}

1 Global Initiative for Asthma. Pocket Guide for Asthma Management and Prevention (for adults and children older than 5 years). 2017.

2 Masoli M, Fabian D, Holt S, Beasley R. The global burden of asthma: executive summary of the GINA Dissemination Committee report. Allergy 2004; 59: 46978.

3 Pearce N, Ait-Khaled N, Beasley R, et al. Worldwide trends in the prevalence of asthma symptoms: phase III of the International Study of Asthma and Allergies in Childhood (ISAAC). Thorax 2007; 62: 758-66.

4 Eder W, Ege MJ, von Mutius E. The Asthma Epidemic. N Engl J Med 2006; 355: 2226-35.

5 Mosmann TR, Cherwinski H, Bond MW, Giedlin MA, Coffman RL. Two types of murine helper $\mathrm{T}$ cell clone. I. Definition according to profiles of lymphokine activities and secreted proteins. J Immunol 1986; 136: 2348-57.

6 Spellberg B, Edwards JE. Type 1/Type 2 Immunity in Infectious Diseases. Clin Infect Dis 2001; 32: 76-102.

7 Fahy J V. Type 2 inflammation in asthma - present in most, absent in many. Nat Rev Immunol 2014; 15: 57-65.

8 Zhang DH, Yang L, Cohn L, et al. Inhibition of allergic inflammation in a murine model of asthma by expression of a dominant-negative mutant of GATA-3. Immunity 1999; 11: 473-82.

9 Wills-Karp M, Luyimbazi J, Xu X, et al. Interleukin-13: central mediator of allergic asthma. Science 1998; 282: 2258-61.

10 Grünig G, Warnock M, Wakil AE, et al. Requirement for IL-13 independently of IL-4 in experimental asthma. Science 1998; 282: 2261-3.

11 Robinson DS, Hamid Q, Ying S, et al. Predominant T ${ }_{\mathrm{H} 2}$-like Bronchoalveolar TLymphocyte Population in Atopic Asthma. N Engl J Med 1992; 326: 298-304.

12 Oliphant CJ, Barlow JL, McKenzie ANJ. Insights into the initiation of type 2 immune responses. Immunology 2011; 134: 378-85.

13 Noben-Trauth N, Hu-Li J, Paul WE. IL-4 secreted from individual naive CD4+ T cells acts in an autocrine manner to induce Th2 differentiation. Eur J Immunol 2002; 32: 1428-33.

14 van Panhuys N, Tang S-C, Prout M, et al. In vivo studies fail to reveal a role for IL4 or STAT6 signaling in Th2 lymphocyte differentiation. Proc Natl Acad Sci U S A 2008; 105: 12423-8.

15 Rael EL, Lockey RF. Interleukin-13 signaling and its role in asthma. World Allergy Organ J 2011; 4: 54-64.

16 Takayama G, Arima K, Kanaji T, et al. Periostin: a novel component of subepithelial fibrosis of bronchial asthma downstream of IL-4 and IL-13 signals. J Allergy Clin Immunol 2006; 118: 98-104. 
17 Oh CK, Raible D, Geba GP, Molfino NA. Biology of the interleukin-9 pathway and its therapeutic potential for the treatment of asthma. Inflamm Allergy Drug Targets 2011; 10: 180-6.

18 Robinson D, Humbert M, Buhl R, et al. Revisiting Type 2-high and Type 2-low airway inflammation in asthma: current knowledge and therapeutic implications. Clin Exp Allergy 2017; 47: 161-75.

19 Hershey GKK. IL-13 receptors and signaling pathways: an evolving web. J Allergy Clin Immunol 2003; 111: 677-90; quiz 691.

20 Leigh R, Ellis R, Wattie J, Donaldson DD, Inman MD. Is Interleukin-13 Critical in Maintaining Airway Hyperresposiveness in Allergen-challenged Mice? Am J Respir Crit Care Med 2004; 170: 851-6.

21 Grunstein MM, Hakonarson $\mathrm{H}$, Leiter J, et al. IL-13-dependent autocrine signaling mediates altered responsiveness of IgE-sensitized airway smooth muscle. Am J Physiol - Lung Cell Mol Physiol 2002; 282: L520-8.

22 Risse P-A, Jo T, Suarez F, et al. Interleukin-13 inhibits proliferation and enhances contractility of human airway smooth muscle cells without change in contractile phenotype. AJP Lung Cell Mol Physiol 2011; 300: L958-66.

23 Pope SM, Brandt EB, Mishra A, et al. IL-13 induces eosinophil recruitment into the lung by an IL-5- and eotaxin-dependent mechanism. J Allergy Clin Immunol 2001; 108: 594-601.

24 Rosenberg HF, Phipps S, Foster PS. Eosinophil trafficking in allergy and asthma. $J$ Allergy Clin Immunol 2007; 119: 1303-10.

25 Warren HS, Kinnear BF, Phillips JH, Lanier LL. Production of IL-5 by human NK cells and regulation of IL-5 secretion by IL-4, IL-10, and IL-12. J Immunol 1995; 154: 5144-52.

26 Tang Y, Zeng Y, Li Y. [Study on mutations of beta chain of high-affinity IgE receptor gene in people of Han nationality of southern China]. Zhonghua Jie He He Hu Xi Za Zhi 2001; 24: 142-4.

27 Bousquet J, Chanez P, Lacoste JY, et al. Eosinophilic inflammation in asthma. N Engl J Med 1990; 323: 1033-9.

28 Barlow JL, McKenzie ANJ. Type-2 innate lymphoid cells in human allergic disease. Curr Opin Allergy Clin Immunol 2014; 14: 397-403.

29 Motomura $\mathrm{Y}$, Kitamura $\mathrm{H}$, Hijikata $\mathrm{A}$, et al. The transcription factor E4BP4 regulates the production of IL-10 and IL-13 in CD4+ T cells. Nat Immunol 2011; 12: 450-9.

30 Green RH, Brightling CE, McKenna S, et al. Asthma exacerbations and sputum eosinophil counts: a randomised controlled trial. Lancet 2002; 360: 1715-21.

31 Green RH, Brightling CE, Woltmann G, Parker D, Wardlaw AJ, Pavord ID. Analysis of induced sputum in adults with asthma: identification of subgroup with isolated sputum neutrophilia and poor response to inhaled corticosteroids. Thorax 2002; 57: 875-9.

32 Beasley R, Semprini A, Mitchell EA. Risk factors for asthma: is prevention possible? Lancet 2015; 386: 1075-85. 
Martino D, Prescott S. Epigenetics and Prenatal Influences on Asthma and Allergic Airways Disease. Chest 2011; 139: 640-7.

34 Tang ML, Kemp AS, Thorburn J, Hill DJ. Reduced interferon-gamma secretion in neonates and subsequent atopy. Lancet 1994; 344: 983-5.

35 Yerkovich ST, Wikström ME, Suriyaarachchi D, Prescott SL, Upham JW, Holt PG. Postnatal Development of Monocyte Cytokine Responses to Bacterial Lipopolysaccharide. Pediatr Res 2007; 62: 547-52.

36 Ohshima Y, Yasutomi M, Omata N, et al. Dysregulation of IL-13 production by cord blood CD4+ $\mathrm{T}$ cells is associated with the subsequent development of atopic disease in infants. Pediatr Res 2002; 51: 195-200.

37 Kusel $\mathrm{MMH}$, de Klerk NH, Kebadze T, et al. Early-life respiratory viral infections, atopic sensitization, and risk of subsequent development of persistent asthma. J Allergy Clin Immunol 2007; 119: 1105-10.

38 Rantala A, Jaakkola JJK, Jaakkola MS. Respiratory Infections Precede Adult-Onset Asthma. PLoS One 2011; 6: e27912.

39 Kerrebijn KF, van Essen-Zandvliet EE, Neijens HJ. Effect of long-term treatment with inhaled corticosteroids and beta-agonists on the bronchial responsiveness in children with asthma. J Allergy Clin Immunol 1987; 79: 653-9.

40 Kraan J, Koëter GH, vd Mark TW, Sluiter HJ, de Vries K. Changes in bronchial hyperreactivity induced by 4 weeks of treatment with antiasthmatic drugs in patients with allergic asthma: a comparison between budesonide and terbutaline. J Allergy Clin Immunol 1985; 76: 628-36.

41 Farquhar H, Crane J, Mitchell EA, Eyers S, Beasley R. The acetaminophen and asthma hypothesis 10 years on: A case to answer. J Allergy Clin Immunol 2009; 124: 649-51.

42 Pearce N, Pekkanen J, Beasley R. How much asthma is really attributable to atopy? Thorax 1999; 54: 268-72.

43 Lin T-Y, Poon AH, Hamid Q. Asthma phenotypes and endotypes. Curr Opin Pulm Med 2013; 19: 18-23.

44 Walker C, Virchow JC, Bruijnzeel PL, Blaser K. T cell subsets and their soluble products regulate eosinophilia in allergic and nonallergic asthma. J Immunol 1991; 146: 1829-35.

45 Lambrecht BN, Hammad H. The immunology of asthma. Nat Immunol 2014; 16 : 45-56.

46 Knudsen TB, Thomsen SF, Nolte H, Backer V. A Population-based Clinical Study of Allergic and Non-allergic Asthma. J Asthma 2009; 46: 91-4.

47 Agache I, Sugita K, Morita H, Akdis M, Akdis CA. The Complex Type 2 Endotype in Allergy and Asthma: From Laboratory to Bedside. Curr Allergy Asthma Rep 2015; 15: 29.

48 Green RH, Pavord I. Stability of inflammatory phenotypes in asthma. Thorax 2012; 67: 665-7.

49 Anderson GP. Endotyping asthma: new insights into key pathogenic mechanisms in a complex, heterogeneous disease. Lancet 2008; 372: 1107-19. 
50 Woodruff PG, Boushey HA, Dolganov GM, et al. Genome-wide profiling identifies epithelial cell genes associated with asthma and with treatment response to corticosteroids. Proc Natl Acad Sci U S A 2007; 104: 15858-63.

51 Woodruff PG, Modrek B, Choy DF, et al. T-helper type 2-driven inflammation defines major subphenotypes of asthma. Am J Respir Crit Care Med 2009; 180: 388-95.

52 Wenzel S, Swanson B, Teper A, et al. Dupilumab reduces severe exacerbations in periostin-high and periostin-low asthma patients. Eur Respir J 2016; 48: OA1798.

53 Corren J, Lemanske RF, Hanania NA, et al. Lebrikizumab Treatment in Adults with Asthma. N Engl J Med 2011; 365: 1088-98.

54 Brightling CE, Chanez P, Leigh R, et al. Efficacy and safety of tralokinumab in patients with severe uncontrolled asthma: a randomised, double-blind, placebocontrolled, phase 2b trial. Lancet Respir Med 2015; 3: 692-701.

55 Ortega HG, Liu MC, Pavord ID, et al. Mepolizumab treatment in patients with severe eosinophilic asthma. N Engl J Med 2014; 371: 1198-207.

56 Hanania NA, Alpan O, Hamilos DL, et al. Omalizumab in severe allergic asthma inadequately controlled with standard therapy: a randomized trial. Ann Intern Med 2011; 154: 573-82.

57 Bisgaard $\mathrm{H}$, Bønnelykke K. Long-term studies of the natural history of asthma in childhood. J Allergy Clin Immunol 2010; 126: 187-97.

58 Martin PE, Matheson MC, Gurrin L, et al. Childhood eczema and rhinitis predict atopic but not nonatopic adult asthma: A prospective cohort study over 4 decades. J Allergy Clin Immunol 2011; 127: 1473-1479.e1.

59 SIMPSON JL, SCOTT R, BOYLE MJ, GIBSON PG. Inflammatory subtypes in asthma: Assessment and identification using induced sputum. Respirology 2006; 11: 5461.

60 Doe C, Bafadhel M, Siddiqui S, et al. Expression of the T Helper 17-Associated Cytokines IL-17A and IL-17F in Asthma and COPD. Chest 2010; 138: 1140-7.

61 Tang ML, Coleman J, Kemp AS. Interleukin-4 and interferon-gamma production in atopic and non-atopic children with asthma. Clin Exp Allergy 1995; 25: 51521.

62 Moore WC, Meyers DA, Wenzel SE, et al. Identification of Asthma Phenotypes Using Cluster Analysis in the Severe Asthma Research Program. Am J Respir Crit Care Med 2010; 181: 315-23.

63 Brusselle GG, VanderStichele C, Jordens P, et al. Azithromycin for prevention of exacerbations in severe asthma (AZISAST): a multicentre randomised doubleblind placebo-controlled trial. Thorax 2013; 68: 322-9.

64 Heaney LG, Djukanovic R, Woodcock A, et al. Research in progress: Medical Research Council United Kingdom Refractory Asthma Stratification Programme (RASP-UK). Thorax 2016; 71: 187-9.

65 Buhl R, Korn S, Menzies-Gow A, et al. Assessing biomarkers in a real-world severe asthma study (ARIETTA). Respir Med 2016; 115: 7-12.

66 Kunicka JE, Talle MA, Denhardt GH, Brown M, Prince LA, Goldstein G. 
Immunosuppression by Glucocorticoids: Inhibition of Production of Multiple Lymphokines by in Vivo Administration of Dexamethasone. Cell Immunol 1993; 149: 39-49.

67 Wu CY, Fargeas C, Nakajima T, Delespesse G. Glucocorticoids suppress the production of interleukin 4 by human lymphocytes. Eur J Immunol 1991; 21: 2645-7.

68 Omalizumab for treating severe persistent allergic asthma | 3-The-technology | Guidance and guidelines | NICE. .

69 Hanania NA, Wenzel S, Rosén K, et al. Exploring the effects of omalizumab in allergic asthma: an analysis of biomarkers in the EXTRA study. Am J Respir Crit Care Med 2013; 187: 804-11.

70 NICE. Asthma (eosinophilic, severe) - mepolizumab [ID798] | Guidance and guidelines | NICE. NICE, 2017.

71 Bleecker ER, FitzGerald JM, Chanez P, et al. Efficacy and safety of benralizumab for patients with severe asthma uncontrolled with high-dosage inhaled corticosteroids and long-acting $\beta 2$-agonists (SIROCCO): a randomised, multicentre, placebo-controlled phase 3 trial. Lancet 2016; 388: 2115-27.

72 Castro M, Zangrilli J, Wechsler ME, et al. Reslizumab for inadequately controlled asthma with elevated blood eosinophil counts: results from two multicentre, parallel, double-blind, randomised, placebo-controlled, phase 3 trials. Lancet Respir Med 2015; 3: 355-66.

73 Taylor DR. Using biomarkers in the assessment of airways disease. J Allergy Clin Immunol 2011; 128: 927-34.

74 Biomarkers Definitions Working Group. Biomarkers and surrogate endpoints: Preferred definitions and conceptual framework. Clin Pharmacol Ther 2001; 69: 89-95.

75 Nomura $\mathrm{H}$, Wada $\mathrm{H}$, Mizuno $\mathrm{T}$, et al. Negative predictive value of d-dimer for diagnosis of venous thromboembolism. Int J Hematol 2008; 87: 250-5.

76 Vukotic V, Cerovic S, Kozomara M, Lazic M. The predictive value of PSA in diagnosis of prostate cancer in non screened population. Acta Chir lugosl 2005; 52: 81-7.

77 Ellis A. The pathological anatomy of bronchial asthma. Am J Med Sci 1908; 136: 407-28.

78 Gleich GJ, Adolphson CR, Leiferman KM. The Biology of the Eosinophilic Leukocyte. Annu Rev Med 1993; 44: 85-101.

79 Ulrik CS. Peripheral eosinophil counts as a marker of disease activity in intrinsic and extrinsic asthma. Clin Exp Allergy 1995; 25: 820-7.

80 Leckie MJ, ten Brinke A, Khan J, et al. Effects of an interleukin-5 blocking monoclonal antibody on eosinophils, airway hyper-responsiveness, and the late asthmatic response. Lancet (London, England); 356: 2144-8.

81 Haldar P, Brightling CE, Hargadon B, et al. Mepolizumab and exacerbations of refractory eosinophilic asthma. N Engl J Med 2009; 360: 973-84.

82 Szefler SJ, Wenzel S, Brown R, et al. Asthma outcomes: biomarkers. J Allergy Clin 
Immunol 2012; 129: S9-23.

83 Pavord ID, Afzalnia S, Menzies-Gow A, Heaney LG. The current and future role of biomarkers in type 2 cytokine-mediated asthma management. Clin Exp Allergy 2017; 47: 148-60.

84 Warke TJ, Fitch PS, Brown V, et al. Exhaled nitric oxide correlates with airway eosinophils in childhood asthma. Thorax 2002; 57: 383-7.

85 Payne DNR, Adcock IM, Wilson NM, Oates T, Scallan M, Bush A. Relationship between Exhaled Nitric Oxide and Mucosal Eosinophilic Inflammation in Children with Difficult Asthma, after Treatment with Oral Prednisolone. Am J Respir Crit Care Med 2001; 164: 1376-81.

86 Mattes J, Storm van's Gravesande K, Reining U, et al. NO in exhaled air is correlated with markers of eosinophilic airway inflammation in corticosteroiddependent childhood asthma. Eur Respir J 1999; 13: 1391-5.

87 Jatakanon A, Lim S, Kharitonov SA, Chung KF, Barnes PJ. Correlation between exhaled nitric oxide, sputum eosinophils, and methacholine responsiveness in patients with mild asthma. Thorax 1998; 53: 91-5.

88 Berry MA, Shaw DE, Green RH, Brightling CE, Wardlaw AJ, Pavord ID. The use of exhaled nitric oxide concentration to identify eosinophilic airway inflammation: an observational study in adults with asthma. Clin Exp Allergy 2005; 35: 1175-9.

89 ATS/ERS recommendations for standardized procedures for the online and offline measurement of exhaled lower respiratory nitric oxide and nasal nitric oxide, 2005. Am J Respir Crit Care Med 2005; 171: 912-30.

90 Kupczyk M, ten Brinke A, Sterk PJ, et al. Frequent exacerbators - a distinct phenotype of severe asthma. Clin Exp Allergy 2014; 44: 212-21.

91 Dweik RA, Boggs PB, Erzurum SC, et al. An official ATS clinical practice guideline: interpretation of exhaled nitric oxide levels (FENO) for clinical applications. Am J Respir Crit Care Med 2011; 184: 602-15.

92 Gemicioglu B, Musellim B, Dogan I, Guven K. Fractional exhaled nitric oxide (FeNo) in different asthma phenotypes. Allergy Rhinol (Providence) 2014; 5: 157-61.

93 Balzar S, Strand M, Rhodes D, Wenzel SE. IgE expression pattern in lung: relation to systemic IgE and asthma phenotypes. J Allergy Clin Immunol 2007; 119: 85562.

94 Tanaka A, Jinno $\mathrm{M}$, Hirai $\mathrm{K}$, et al. Longitudinal increase in total IgE levels in patients with adult asthma: an association with poor asthma control. Respir Res 2014; 15: 144.

95 Hamilton RG, Matsson PN, Hovanec-Burns DL, et al. Analytical Performance Characteristics, Quality Assurance and Clinical Utility of Immunological Assays for Human IgE Antibodies of Defined Allergen Specificities. (CLSI-ILA20-A3). J Allergy Clin Immunol 2015; 135: AB8.

96 Bousquet J, Rabe $\mathrm{K}$, Humbert $\mathrm{M}$, et al. Predicting and evaluating response to omalizumab in patients with severe allergic asthma. Respir Med 2007; 101: 1483-92. 
97 Kanemitsu Y, Matsumoto $\mathrm{H}$, Izuhara K, et al. Increased periostin associates with greater airflow limitation in patients receiving inhaled corticosteroids. J Allergy Clin Immunol 2013; 132: 305-12.e3.

98 Jia G, Erickson RW, Choy DF, et al. Periostin is a systemic biomarker of eosinophilic airway inflammation in asthmatic patients. J Allergy Clin Immunol 2012; 130: 647-654.e10.

99 Wagener AH, de Nijs SB, Lutter R, et al. External validation of blood eosinophils, $\mathrm{FE}(\mathrm{NO})$ and serum periostin as surrogates for sputum eosinophils in asthma. Thorax 2015; 70: 115-20.

100 Fingleton J, Braithwaite I, Travers J, et al. Serum periostin in obstructive airways disease. Eur Respir J 2016; 47: 1383-91.

101 Johansson MW, Evans MD, Crisafi GM, Holweg CTJ, Matthews JG, Jarjour NN. Serum periostin is associated with type 2 immunity in severe asthma. J Allergy Clin Immunol 2016; 137: 1904-1907.e2.

102 Barbee RA, Halonen M, Lebowitz M, Burrows B. Distribution of $\lg E$ in a community population sample: correlations with age, sex, and allergen skin test reactivity. J Allergy Clin Immunol 1981; 68: 106-11.

103 Conway SJ, Izuhara K, Kudo Y, et al. The role of periostin in tissue remodeling across health and disease. Cell Mol Life Sci 2014; 71: 1279-88.

104 Horiuchi K, Amizuka N, Takeshita S, et al. Identification and characterization of a novel protein, periostin, with restricted expression to periosteum and periodontal ligament and increased expression by transforming growth factor beta. J Bone Miner Res 1999; 14: 1239-49.

105 Takeshita S, Kikuno R, Tezuka K, Amann E. Osteoblast-specific factor 2: cloning of a putative bone adhesion protein with homology with the insect protein fasciclin I. Biochem J 1993; 294 ( Pt 1: 271-8.

106 Merle B, Garnero P. The multiple facets of periostin in bone metabolism. Osteoporos Int 2012; 23: 1199-212.

107 Norris RA, Moreno-Rodriguez R, Hoffman S, Markwald RR. The many facets of the matricelluar protein periostin during cardiac development, remodeling, and pathophysiology. J Cell Commun Signal 2009; 3: 275-86.

108 Elkins T, Hortsch M, Bieber AJ, Snow PM, Goodman CS. Drosophila fasciclin I is a novel homophilic adhesion molecule that along with fasciclin III can mediate cell sorting. J Cell Biol 1990; 110: 1825-32.

109 Snider P, Hinton RB, Moreno-Rodriguez RA, et al. Periostin is required for maturation and extracellular matrix stabilization of noncardiomyocyte lineages of the heart. Circ Res 2008; 102: 752-60.

110 Bonnet N, Standley KN, Bianchi EN, et al. The matricellular protein periostin is required for sost inhibition and the anabolic response to mechanical loading and physical activity. J Biol Chem 2009; 284: 35939-50.

111 Uchida M, Shiraishi H, Ohta S, et al. Periostin, a Matricellular Protein, Plays a Role in the Induction of Chemokines in Pulmonary Fibrosis. Am J Respir Cell Mol Biol 2012; 46: 677-86. 
112 Habernau Mena A, Del Pozo Abejón V, Rodríguez Vidigal F, Bobadilla González P. Role of Periostin in Uncontrolled Asthma in Children (DADO study). J Investig Allergol Clin Immunol 2017; 27: 291-8.

113 Takayama I, Kudo A. Periostin in dental science. Jpn Dent Sci Rev 2012; 48: 92-8.

114 Shimazaki M, Nakamura K, Kii I, et al. Periostin is essential for cardiac healingafter acute myocardial infarction. J Exp Med 2008; 205: 295-303.

115 Palme S, Christenson RH, Jortani SA, et al. Multicenter evaluation of analytical characteristics of the Elecsys $\left({ }^{\oplus}\right)$ Periostin immunoassay. Clin Biochem 2017; 50: 139-44.

116 Zhu S, Barbe MF, Liu C, et al. Periostin-like-factor in osteogenesis. J Cell Physiol 2009; 218: 584-92.

117 Rios $\mathrm{H}$, Koushik S V, Wang $\mathrm{H}$, et al. Periostin null mice exhibit dwarfism, incisor enamel defects, and an early-onset periodontal disease-like phenotype. Mol Cell Biol 2005; 25: 11131-44.

118 Zhu S, Barbe MF, Amin N, et al. Immunolocalization of Periostin-like factor and Periostin during embryogenesis. J Histochem Cytochem 2008; 56: 329-45.

119 Nakazawa T, Nakajima A, Seki N, et al. Gene expression of periostin in the early stage of fracture healing detected by cDNA microarray analysis. J Orthop Res 2004; 22: 520-5.

120 Maruhashi T, Kii I, Saito M, Kudo A. Interaction between Periostin and BMP-1 Promotes Proteolytic Activation of Lysyl Oxidase. J Biol Chem 2010; 285: 13294303.

121 Ontsuka K, Kotobuki $\mathrm{Y}$, Shiraishi $\mathrm{H}$, et al. Periostin, a matricellular protein, accelerates cutaneous wound repair by activating dermal fibroblasts. Exp Dermatol 2012; 21: 331-6.

122 Rani S, Barbe MF, Barr AE, Litvin J. Periostin-like-factor and Periostin in an animal model of work-related musculoskeletal disorder. Bone 2009; 44: 502-12.

123 Jackson-Boeters L, Wen W, Hamilton DW. Periostin localizes to cells in normal skin, but is associated with the extracellular matrix during wound repair. J Cell Commun Signal 2009; 3: 125-33.

124 Nishiyama T, Kii I, Kashima TG, et al. Delayed Re-Epithelialization in PeriostinDeficient Mice during Cutaneous Wound Healing. PLoS One 2011; 6: e18410.

125 Elliott CG, Wang J, Guo X, et al. Periostin modulates myofibroblast differentiation during full-thickness cutaneous wound repair. J Cell Sci 2012; 125: 121-32.

126 Ruan K, Bao S, Ouyang G. The multifaceted role of periostin in tumorigenesis. Cell Mol Life Sci 2009; 66: 2219-30.

127 Siriwardena BSMS, Kudo Y, Ogawa I, et al. Periostin is frequently overexpressed and enhances invasion and angiogenesis in oral cancer. Br J Cancer 2006; 95 : 1396-403.

128 Bao S, Ouyang G, Bai X, et al. Periostin potently promotes metastatic growth of colon cancer by augmenting cell survival via the Akt/PKB pathway. Cancer Cell 2004; 5: 329-39. 
129 Shao R, Bao S, Bai X, et al. Acquired expression of periostin by human breast cancers promotes tumor angiogenesis through up-regulation of vascular endothelial growth factor receptor 2 expression. Mol Cell Biol 2004; 24: 39924003.

130 Kikuchi Y, Kashima TG, Nishiyama T, et al. Periostin Is Expressed in Pericryptal Fibroblasts and Cancer-associated Fibroblasts in the Colon. J Histochem Cytochem 2008; 56: 753-64.

131 Kudo Y, Ogawa I, Kitajima S, et al. Periostin Promotes Invasion and AnchorageIndependent Growth in the Metastatic Process of Head and Neck Cancer. Cancer Res 2006; 66: 6928-35.

132 Deraz EM, Kudo Y, Yoshida M, et al. MMP-10/Stromelysin-2 Promotes Invasion of Head and Neck Cancer. PLoS One 2011; 6: e25438.

133 Kudo Y, lizuka S, Yoshida M, et al. Periostin Directly and Indirectly Promotes Tumor Lymphangiogenesis of Head and Neck Cancer. PLoS One 2012; 7: e44488.

134 Conway SJ, Molkentin JD. Periostin as a heterofunctional regulator of cardiac development and disease. Curr Genomics 2008; 9: 548-55.

135 Kühn B, del Monte F, Hajjar RJ, et al. Periostin induces proliferation of differentiated cardiomyocytes and promotes cardiac repair. Nat Med 2007; 13 : 962-9.

136 Hakuno D, Kimura N, Yoshioka M, et al. Periostin advances atherosclerotic and rheumatic cardiac valve degeneration by inducing angiogenesis and MMP production in humans and rodents. J Clin Invest 2010; 120: 2292-306.

137 Stansfield WE, Andersen NM, Tang R-H, Selzman CH. Periostin is a novel factor in cardiac remodeling after experimental and clinical unloading of the failing heart. Ann Thorac Surg 2009; 88: 1916-21.

138 Asakura M, Kitakaze M. Global gene expression profiling in the failing myocardium. Circ J 2009; 73: 1568-76.

139 Hixson JE, Shimmin LC, Montasser ME, et al. Common variants in the periostin gene influence development of atherosclerosis in young persons. Arterioscler Thromb Vasc Biol 2011; 31: 1661-7.

140 Conway SJ, Doetschman T, Azhar M. The inter-relationship of periostin, TGF beta, and BMP in heart valve development and valvular heart diseases. ScientificWorldJournal 2011; 11: 1509-24.

141 Satirapoj B, Wang Y, Chamberlin MP, et al. Periostin: novel tissue and urinary biomarker of progressive renal injury induces a coordinated mesenchymal phenotype in tubular cells. Nephrol Dial Transplant 2012; 27: 2702-11.

142 Sorocos K, Kostoulias X, Cullen-McEwen L, Hart AH, Bertram JF, Caruana G. Expression patterns and roles of periostin during kidney and ureter development. J Urol 2011; 186: 1537-44.

143 Guerrot D, Dussaule J-C, Mael-Ainin M, et al. Identification of periostin as a critical marker of progression/reversal of hypertensive nephropathy. 2012; 7: e31974.

144 Bozyk PD, Bentley JK, Popova AP, et al. Neonatal periostin knockout mice are 
protected from hyperoxia-induced alveolar simplification. PLoS One 2012; 7: e31336.

145 Sidhu SS, Yuan S, Innes a. L, et al. Roles of epithelial cell-derived periostin in TGF- activation, collagen production, and collagen gel elasticity in asthma. Proc Natl Acad Sci 2010; 107: 14170-5.

146 Naik PK, Bozyk PD, Bentley JK, et al. Periostin promotes fibrosis and predicts progression in patients with idiopathic pulmonary fibrosis. Am J Physiol Lung Cell Mol Physiol 2012; 303: L1046-56.

147 Okamoto $\mathrm{M}$, Hoshino $\mathrm{T}$, Kitasato $\mathrm{Y}$, et al. Periostin, a matrix protein, is a novel biomarker for idiopathic interstitial pneumonias. Eur Respir J 2011; 37: 1119-27.

148 Johansson MW, Annis DS, Mosher DF. $\alpha_{M} \beta_{2}$ Integrin-Mediated Adhesion and Motility of IL-5-Stimulated Eosinophils on Periostin. Am J Respir Cell Mol Biol 2013; 48: 503-10.

149 Nagasaki T, Matsumoto H, Kanemitsu Y, et al. Integrating longitudinal information on pulmonary function and inflammation using asthma phenotypes. J Allergy Clin Immunol 2014; 133: 1474-7, 1477.e1-2.

150 Nagasaki T, Matsumoto $\mathrm{H}$, Kanemitsu $\mathrm{Y}$, et al. Using exhaled nitric oxide and serum periostin as a composite marker to identify severe/steroid-insensitive asthma. Am J Respir Crit Care Med 2014; 190: 1449-52.

151 Favus M. Primer on the Metabolic Bone Diseases and Disorders of Mineral Metabolism., 3rd Editio. Philadelphia, PA, USA: Lippincott-Raven, Philadelphia, PA, USA, 1996.

152 Robey P. Bone maxtrix proteoglycans and glycoproteins. In: Bilezikian, JP, Raisz, LG, Rodan G, ed. Principles of Bone Biology. Academic Press, San Diego, CA, USA, 1996: 155-65.

153 Ueno T, Kagawa T, Mizukawa N, Nakamura H, Sugahara T, Yamamoto T. Cellular origin of endochondral ossification from grafted periosteum. Anat Rec 2001; 264: 348-57.

154 Fang J, Hall BK. Chondrogenic cell differentiation from membrane bone periostea. Anat Embryol (Berl) 1997; 196: 349-62.

155 Simmons DJ. Fracture healing perspectives. Clin Orthop Relat Res 1985; : 10013.

156 Sawakami K, Robling AG, Ai M, et al. The Wnt Co-receptor LRP5 Is Essential for Skeletal Mechanotransduction but Not for the Anabolic Bone Response to Parathyroid Hormone Treatment. J Biol Chem 2006; 281: 23698-711.

157 Robling AG, Niziolek PJ, Baldridge LA, et al. Mechanical Stimulation of Bone in Vivo Reduces Osteocyte Expression of Sost/Sclerostin. J Biol Chem 2007; 283: 5866-75.

158 Izbicka E, Dunstan CR, Horn D, et al. Effects of human tumor cell lines on local new bone formation in vivo. Calcif Tissue Int 1997; 60: 210-5.

159 de Souza RL, Pitsillides AA, Lanyon LE, Skerry TM, Chenu C. Sympathetic Nervous System Does Not Mediate the Load-Induced Cortical New Bone Formation. J Bone Miner Res 2005; 20: 2159-68. 
160 Chambers TJ, Evans M, Gardner TN, Turner-Smith A, Chow JW. Induction of bone formation in rat tail vertebrae by mechanical loading. Bone Miner 1993; 20: 167-78.

161 Nanci A. Ten Cate's oral histology: development, structure, and function., 6th Ed. St Louis, MO, USA: Mosby, 2003.

162 Lekic $\mathrm{P}, \mathrm{McC}$ (lloch CAG. Periodontal ligament cell populations: The central role of fibroblasts in creating a unique tissue. Anat Rec 1996; 245: 327-41.

163 Kruzynska-Frejtag A, Wang J, Maeda M, et al. Periostin is expressed within the developing teeth at the sites of epithelial-mesenchymal interaction. Dev Dyn 2004; 229: 857-68.

164 Wilde J, Yokozeki M, Terai K, Kudo A, Moriyama K. The divergent expression of periostin mRNA in the periodontal ligament during experimental tooth movement. Cell Tissue Res 2003; 312: 345-51.

165 Bornstein P. Matricellular proteins: an overview. Matrix Biol 2000; 19: 555-6.

166 Ferris BG. Epidemiology Standardization Project (American Thoracic Society). Am Rev Respir Dis 1978; 118: 1-120.

167 Marsell R, Einhorn TA. The biology of fracture healing. Injury 2011; 42: 551-5.

168 Rosselli-Murai LK, Almeida LO, Zagni C, et al. Periostin responds to mechanical stress and tension by activating the MTOR signaling pathway. PLoS One 2013; 8: e83580.

169 Cottrell J, O'Connor JP. Effect of Non-Steroidal Anti-Inflammatory Drugs on Bone Healing. Pharmaceuticals (Basel) 2010; 3: 1668-93.

170 Bonnet N, Biver E, Durosier C, Chevalley T, Rizzoli R, Ferrari S. Additive Genetic Effects on Circulating Periostin Contribute to the Heritability of Bone Microstructure. J Clin Endocrinol Metab 2015; 100: E1014-21.

171 Semprini R, Caswell-Smith R, Fingleton J, et al. Longitudinal variation of serum periostin levels in adults with stable asthma. J Allergy Clin Immunol 2017; 139: 1687-8.

172 Padial-Molina M, Volk SL, Taut AD, Giannobile W V, Rios HF. Periostin is downregulated during periodontal inflammation. J Dent Res 2012; 91: 1078-84.

173 Matsusaka M, Kabata H, Fukunaga K, et al. Phenotype of asthma related with high serum periostin levels. Allergol Int 2015; 64: 175-80.

174 Caswell-Smith R, Hosking A, Cripps T, et al. Reference ranges for serum periostin in a population without asthma or COPD. Clin Exp Allergy 2016; 46: 1303-14.

175 Wempe JB, Tammeling EP, Koëter GH, Håkansson L, Venge P, Postma DS. Blood eosinophil numbers and activity during 24 hours: effects of treatment with budesonide and bambuterol. J Allergy Clin Immunol 1992; 90: 757-65.

176 Panzer SE, Dodge AM, Kelly EAB, Jarjour NN. Circadian variation of sputum inflammatory cells in mild asthma. J Allergy Clin Immunol 2003; 111: 308-12.

177 Kelly EAB, Houtman JJ, Jarjour NN. Inflammatory changes associated with circadian variation in pulmonary function in subjects with mild asthma. Clin Exp Allergy 2004; 34: 227-33. 
178 Winkel P, Statland BE, Saunders AM, Osborn H, Kupperman H. Within-day physiologic variation of leukocyte types in healthy subjects as assayed by two automated leukocyte differential analyzers. Am J Clin Pathol 1981; 75: 693-700.

179 Uhrbrand $\mathrm{H}$. The number of circulating eosinophils; normal figures and spontaneous variations. Acta Med Scand 1958; 160: 99-104.

180 Holgate ST. The epidemic of allergy and asthma. Nature 1999; 402: B2-4.

181 Singh AM, Busse WW. Asthma exacerbations. 2: aetiology. Thorax 2006; 61: 809-16.

182 Juniper EF, Bousquet J, Abetz L, Bateman ED, GOAL Committee. Identifying 'wellcontrolled' and 'not well-controlled' asthma using the Asthma Control Questionnaire. Respir Med 2006; 100: 616-21.

183 Juniper EF, Guyatt GH, Epstein RS, Ferrie PJ, Jaeschke R, Hiller TK. Evaluation of impairment of health related quality of life in asthma: development of a questionnaire for use in clinical trials. Thorax 1992; 47: 76-83.

184 Miller MR, Hankinson J, Brusasco V, et al. Standardisation of spirometry. Eur Respir J 2005; 26: 319-38.

185 Quanjer PH, Stanojevic S, Cole TJ, et al. Multi-ethnic reference values for spirometry for the 3-95-yr age range: the global lung function 2012 equations. Eur Respir J 2012; 40: 1324-43.

186 Sine H, Zakowski J, Horowitz GL, Altaie S, Boyd JC, Ceriotti F, Garg U, Horn P PA. EP28-A3c: Defining, Establishing, and Verifying Reference Intervals in the Clinical Laboratory; Approved Guideline-Third Edition. Clin Lab Stand Inst 2010. DOI:56238-682-4.

187 Hanania NA, Noonan M, Corren J, et al. Lebrikizumab in moderate-to-severe asthma: pooled data from two randomised placebo-controlled studies. Thorax 2015; 70: 748-56.

188 Arron JR, Izuhara K. Asthma biomarkers: what constitutes a 'gold standard'? Thorax 2015; 70: 105-7.

189 Malinovschi A, Fonseca JA, Jacinto T, Alving K, Janson C. Exhaled nitric oxide levels and blood eosinophil counts independently associate with wheeze and asthma events in National Health and Nutrition Examination Survey subjects. J Allergy Clin Immunol 2013; 132: 821-7.e1-5.

190 Matsumoto $\mathrm{H}$. Serum periostin: a novel biomarker for asthma management. Allergol Int 2014; 63: 153-60.

191 Litonjua AA, Celedón JC, Hausmann J, et al. Variation in total and specific IgE: effects of ethnicity and socioeconomic status. J Allergy Clin Immunol 2005; 115: 751-7.

192 Gergen PJ, Arbes SJ, Calatroni A, Mitchell HE, Zeldin DC. Total IgE levels and asthma prevalence in the US population: results from the National Health and Nutrition Examination Survey 2005-2006. J Allergy Clin Immunol 2009; 124: 44753.

193 Court CS, Cook DG, Strachan DP. The descriptive epidemiology of house dust mite-specific and total immunoglobin $E$ in England using a nationally 
representative sample. Clin Exp Allergy 2002; 32: 1033-41.

194 Oryszczyn M-P, Annesi-Maesano I, Charpin D, Paty E, Maccario J, Kauffmann F. Relationships of Active and Passive Smoking to Total IgE in Adults of the Epidemiological Study of the Genetics and Environment of Asthma, Bronchial Hyperresponsiveness, and Atopy (EGEA). Am J Respir Crit Care Med 2000; 161: 1241-6.

195 Bain B, Seed M, Godsland I. Normal values for peripheral blood white cell counts in women of four different ethnic origins. J Clin Pathol 1984; 37: 188-93.

196 Brody DJ, Zhang X, Kit BK, Dillon CF. Reference values and factors associated with exhaled nitric oxide: U.S. youth and adults. Respir Med 2013; 107: 1682-91.

197 Linn WS, Rappaport EB, Berhane KT, Bastain TM, Avol EL, Gilliland FD. Exhaled nitric oxide in a population-based study of southern California schoolchildren. Respir Res 2009; 10: 28.

198 Wong GWK, Liu EKH, Leung TF, et al. High levels and gender difference of exhaled nitric oxide in Chinese schoolchildren. Clin Exp Allergy 2005; 35: 889-93.

199 Sonnappa S, Bastardo CM, Stafler P, Bush A, Aurora P, Stocks J. Ethnic differences in fraction of exhaled nitric oxide and lung function in healthy young children. Chest 2011; 140: 1325-31.

200 Caswell-Smith R, Cripps T, Charles T, Hoskin A, Holweg C, Matthews J, Holliday M, Maillot C, Fingleton J, Weatherall M, Braithwaite I BR. Day-time variation of serum periostin in adults with and without asthma. Allergy, Asthma Clin Immunol 2017.

201 Yates DH, Kharitonov SA, Robbins RA, Thomas PS, Barnes PJ. Effect of a nitric oxide synthase inhibitor and a glucocorticosteroid on exhaled nitric oxide. Am J Respir Crit Care Med 1995; 152: 892-6.

202 Ortega HG, Yancey SW, Mayer B, et al. Severe eosinophilic asthma treated with mepolizumab stratified by baseline eosinophil thresholds: a secondary analysis of the DREAM and MENSA studies. Lancet Respir Med 2016; 4: 549-56.

203 European Medicines Agency. Annex I: Summary of product characteristics, Xolair. 2005.

204 Dougherty RH, Fahy J V. Acute exacerbations of asthma: epidemiology, biology and the exacerbation-prone phenotype. Clin Exp Allergy 2009; 39: 193-202.

205 Denlinger LC, Phillips BR, Ramratnam S, et al. Inflammatory and Comorbid Features of Patients with Severe Asthma and Frequent Exacerbations. Am J Respir Crit Care Med 2017; 195: 302-13.

2062017 GINA Report: Global Strategy for Asthma Management and Prevention | Global Initiative for Asthma - GINA. 2017. http://ginasthma.org/2017-ginareport-global-strategy-for-asthma-management-and-prevention/ (accessed July 21, 2017).

207 Pavord ID, Korn S, Howarth P, et al. Mepolizumab for severe eosinophilic asthma (DREAM): a multicentre, double-blind, placebo-controlled trial. Lancet (London, England) 2012; 380: 651-9.

208 Teach SJ, Gergen PJ, Szefler SJ, et al. Seasonal risk factors for asthma 
exacerbations among inner-city children. J Allergy Clin Immunol 2015; 135 : 1465-73.e5.

209 Reddel HK, Taylor DR, Bateman ED, et al. An official American Thoracic Society/European Respiratory Society statement: asthma control and exacerbations: standardizing endpoints for clinical asthma trials and clinical practice. Am J Respir Crit Care Med 2009; 180: 59-99.

210 Altman LC, Hill JS, Hairfield WM, Mullarkey MF. Effects of Corticosteroids on Eosinophil Chemotaxis and Adherence. J Clin Invest 1981; 67: 28-36.

211 FDA, CDER. Prescribing information for NUCALA (mepolizumab). 2015. https://www.accessdata.fda.gov/drugsatfda_docs/label/2015/1255260rig1s000 Lbl.pdf.

212 Kharitonov SA, Donnelly LE, Montuschi P, Corradi M, Collins J V, Barnes PJ. Dosedependent onset and cessation of action of inhaled budesonide on exhaled nitric oxide and symptoms in mild asthma. Thorax 2002; 57: 889-96.

213 Silkoff PE, McClean P, Spino M, Erlich L, Slutsky AS, Zamel N. Dose-response relationship and reproducibility of the fall in exhaled nitric oxide after inhaled beclomethasone dipropionate therapy in asthma patients. Chest 2001; 119: 1322-8.

214 Matsunaga K, Hirano T, Akamatsu K, Minakata Y. Predictors for identifying the efficacy of systemic steroids on sustained exhaled nitric oxide elevation in severe asthma. Allergol Int 2013; 62: 359-65.

215 Zieg G, Lack G, Harbeck RJ, Gelfand EW, Leung DY. In vivo effects of glucocorticoids on IgE production. J Allergy Clin Immunol 1994; 94: 222-30.

216 Settipane GA, Pudupakkam RK, McGowan JH. Corticosteroid effect on immunoglobulins. J Allergy Clin Immunol 1978; 62: 162-6.

217 Paul-Eugène N, Kolb JP, Damais $C$, et al. Beta 2-adrenoceptor agonists regulate the IL-4-induced phenotypical changes and IgE-dependent functions in normal human monocytes. J Leukoc Biol 1994; 55: 313-20.

218 Tajiri T, Matsumoto H, Gon Y, et al. Utility of serum periostin and free IgE levels in evaluating responsiveness to omalizumab in patients with severe asthma. Allergy 2016; 71: 1472-9.

219 Chung SM. Clinical Pharmacology Review for Xolair (Omalizumab). 2008. https:// www.fda.gov/downloads/drugs/developmentapprovalprocess/developmentres ources/ucm202181.pdf.

220 Fingleton J, Travers J, Williams M, et al. Treatment responsiveness of phenotypes of symptomatic airways obstruction in adults. J Allergy Clin Immunol 2015; 136: 601-9.

221 Zeiger RS, Schatz M, Li Q, et al. High Blood Eosinophil Count Is a Risk Factor for Future Asthma Exacerbations in Adult Persistent Asthma. J Allergy Clin Immunol Pract 2014; 2: 741-750.e4.

222 Malinovschi A, Janson C, Borres M, Alving K. Simultaneously increased fraction of exhaled nitric oxide levels and blood eosinophil counts relate to increased asthma morbidity. J Allergy Clin Immunol 2016; 138: 1301-1308.e2. 
223 Schleich FN, Chevremont A, Paulus V, et al. Importance of concomitant local and systemic eosinophilia in uncontrolled asthma. Eur Respir J 2014; 44: 97-108.

224 Anderson WJ, Short PM, Williamson PA, Manoharan A, Lipworth BJ. The inverse agonist propranolol confers no corticosteroid-sparing activity in mild-tomoderate persistent asthma. Clin Sci 2014; 127: 635-43.

225 Suissa S, Ernst P, Benayoun S, Baltzan M, Cai B. Low-Dose Inhaled Corticosteroids and the Prevention of Death from Asthma. N Engl J Med 2000; 343: 332-6.

226 Donahue JG, Weiss ST, Livingston JM, Goetsch MA, Greineder DK, Platt R. Inhaled steroids and the risk of hospitalization for asthma. JAMA 1997; 277: 887-91.

227 Berry M, Morgan A, Shaw DE, et al. Pathological features and inhaled corticosteroid response of eosinophilic and non-eosinophilic asthma. Thorax 2007; 62: 1043-9.

228 Martin MJ, Wilson E, Gerrard-Tarpey W, et al. The utility of exhaled nitric oxide in patients with suspected asthma. Thorax 2016; 71: 562-4.

229 Brightling CE, George L. Is the eosinophil a leading villain in lung function decline? Chest 2015; 148: 844-6.

230 van Veen $\mathrm{IH}$, ten Brinke $\mathrm{A}$, Sterk PJ, et al. Exhaled nitric oxide predicts lung function decline in difficult-to-treat asthma. Eur Respir J 2008; 32: 344-9.

231 Wenzel SE. Asthma phenotypes: the evolution from clinical to molecular approaches. Nat Med 2012; 18: 716-25.

232 Fahy J V, Kim KW, Liu J, Boushey HA. Prominent neutrophilic inflammation in sputum from subjects with asthma exacerbation. J Allergy Clin Immunol 1995; 95: 843-52.

233 Cowan DC, Taylor DR, Peterson LE, et al. Biomarker-based asthma phenotypes of corticosteroid response. J Allergy Clin Immunol 2015; 135: 877-83.e1.

234 Sur S, Crotty TB, Kephart GM, et al. Sudden-Onset Fatal Asthma: A Distinct Entity with Few Eosinophils and Relatively More Neutrophils in the Airway Submucosa? Am Rev Respir Dis 1993; 148: 713-9.

235 McGrath KW, Icitovic N, Boushey HA, et al. A Large Subgroup of Mild-toModerate Asthma Is Persistently Noneosinophilic. Am J Respir Crit Care Med 2012; 185: 612-9.

236 Loza MJ, Djukanovic R, Chung KF, et al. Validated and longitudinally stable asthma phenotypes based on cluster analysis of the ADEPT study. Respir Res 2016; 17: 165.

237 Silkoff PE, Laviolette M, Singh D, et al. Identification of airway mucosal type 2 inflammation by using clinical biomarkers in asthmatic patients. J Allergy Clin Immunol 2017; published online Jan 13. DOI:10.1016/j.jaci.2016.11.038.

238 Morishita R, Taniyama $\mathrm{Y}$, Katsuragi $\mathrm{N}$, et al. Role of periostin in cancer progression and metastasis: Inhibition of breast cancer progression and metastasis by anti-periostin antibody in a murine model. Int J Mol Med 2011; 28: 181-6. 
239 Masuoka M, Shiraishi $\mathrm{H}$, Ohta $\mathrm{S}$, et al. Periostin promotes chronic allergic inflammation in response to Th2 cytokines. J Clin Invest 2012; 122: 2590-600.

240 Gordon ED, Sidhu SS, Wang Z-E, et al. A protective role for periostin and TGF- $\beta$ in IgE-mediated allergy and airway hyperresponsiveness. Clin Exp Allergy 2012; 42: $144-55$.

241 Ingram JL, Kraft M. IL-13 in asthma and allergic disease: Asthma phenotypes and targeted therapies. J Allergy Clin Immunol 2012; 130: 829-42.

242 Holt S, Beasley R. The Burden of Asthma in New Zealand. Asthma Found. New Zeal. 2002.

243 Brown HM. Treatment of chronic asthma with prednisolone; significance of eosinophils in the sputum. Lancet (London, England) 1958; 2: 1245-7.

244 Nair P, Kraft M. Serum periostin as a marker of $T(H) 2$-dependent eosinophilic airway inflammation. J Allergy Clin Immunol 2012; 130: 655-6.

245 McClintock D, Corren J, Hanania NA, et al. Lebrikizumab, An Anti-IL-13 Monoclonal Antibody, Reduces Severe Asthma Exacerbations Over 32 Weeks In Adults With Inadequately Controlled Asthma. In: C22. ASTHMA THERAPY. American Thoracic Society, 2012: A3959-A3959. 